University of Louisville

ThinkIR: The University of Louisville's Institutional Repository

8-2018

\title{
Perinatal nicotine with or without early life influenza infection leads to lung dysfunction with age.
}

Ryan Carroll McAllister

University of Louisville

Follow this and additional works at: https://ir.library.louisville.edu/etd

Part of the Respiratory Tract Diseases Commons, and the Translational Medical Research Commons

\section{Recommended Citation}

McAllister, Ryan Carroll, "Perinatal nicotine with or without early life influenza infection leads to lung dysfunction with age." (2018). Electronic Theses and Dissertations. Paper 3049.

https://doi.org/10.18297/etd/3049

This Doctoral Dissertation is brought to you for free and open access by ThinkIR: The University of Louisville's Institutional Repository. It has been accepted for inclusion in Electronic Theses and Dissertations by an authorized administrator of ThinkIR: The University of Louisville's Institutional Repository. This title appears here courtesy of the author, who has retained all other copyrights. For more information, please contact thinkir@louisville.edu. 
PERINATAL NICOTINE WITH OR WITHOUT EARLY LIFE INFLUENZA INFECTION LEADS TO LUNG DYSFUNCTION WITH AGE

\author{
By \\ Ryan Carroll McAllister \\ B.S., Northern Michigan University, 2010 \\ M.S., University of Louisville, 2014

\begin{abstract}
A Dissertation
Submitted to the Faculty of the

School of Medicine of the University of Louisville

In Partial Fulfillment of the Requirements for the Degree of
\end{abstract} \\ Doctor of Philosophy in Pharmacology and Toxicology \\ Department of Pharmacology and Toxicology \\ University of Louisville \\ Louisville, Kentucky
}

August 2018 

PERINATAL NICOTINE WITH OR WITHOUT EARLY LIFE INFLUENZA INFECTION LEADS TO LUNG DYSFUNCTION WITH AGE

\author{
By \\ Ryan Carroll McAllister \\ B.S., Northern Michigan University, 2010 \\ M.S., University of Louisville, 2014 \\ A Dissertation Approved on
}

June $29^{\text {th }}, 2018$

By the following Dissertation Committee

\begin{tabular}{c}
\hline $\begin{array}{c}\text { Jesse Roman } \\
\text { (Dissertation Director) }\end{array}$ \\
\hline Kenneth Palmer \\
\hline Igor Lukashevich \\
\hline Gary Hoyle \\
\hline Donghoon Chung
\end{tabular}




\section{ACKNOWLEDGMENTS}

I would like to first acknowledge Dr. Jesse Roman and his laboratory staff, most notably Jeff Ritzenthaler and Edilson Torres-Gonzalez for their aid in helping me complete my Ph.D. project within a constricted time frame. Dr. Roman's attentiveness and disposition have been vital in the completion of my degree, to the point where I would say he is second to no one at the University of Louisville. Before joining Dr. Roman, I was working under a different P.I. who decided to leave the University, two years into my Ph.D. candidacy. With having the rug pulled from underneath my feet, and not knowing how to proceed with my academic career, Dr. Roman suggested that we collaborate our skills (to which I am personally indebted). For his acceptance of my situation and aid in completing my degree I am forever indebted. Furthermore, his staff has been greatly helpful in aiding the experiments conducted pertaining to my dissertation.

In addition, I would like to acknowledge my family for their continued support and advice during my graduate career. Although their understanding for the field is limited, they have always been there to provide guidance to my decisions by having an open ear and mind. Being the first to receive a doctorate in my family is credit to my immediate family and extended instilling the attributes of persistence, working hard in an intellectual manner, and believing in myself. For their guidance and support, through my upbringing and throughout my academic career, I am extremely grateful. Lastly, to all the teachers and professors that have educated me from kindergarten onward, thank you. 


\title{
ABSTRACT \\ PERINATAL NICOTINE WITH OR WITHOUT EARLY LIFE INFLUENZA INFECTION LEADS TO LUNG DYSFUNCTION WITH AGE
}

\author{
Ryan C. McAllister
}

June $29^{\text {th }}, 2018$

Obstructive Airway Diseases (OADs) affect millions of people worldwide, and are characterized by chronic inflammation and tissue remodeling in the lung. It has been proposed that the development of OAD is greatly influenced (and perhaps pre-determined) by early life events, such as maternal smoking or a viral infection. However, direct evidence of this is limited and the mechanisms involved remain unclear. Our laboratory previously developed a murine model of perinatal nicotine exposure, and reported that nicotine leads to airway remodeling and decreased pulmonary function in the offspring. We discovered these effects were mediated through the $\alpha 7$ nicotinic acetylcholine receptor (nAChR). Similarly, respiratory viral infections during childhood, such as Influenza A Virus (IAV), have been implicated in the development of OADs in epidemiological and murine studies. We set out to investigate if perinatal nicotine and/or early life IAV infection could promote lung remodeling and long-term pulmonary dysfunction. We employed a previously established murine model for nicotine exposure and a newly developed model for early life IAV infection. Importantly, lung dysfunction was tested at 7-months of age, in which all other studies look at lung dysfunction at earlier times. 
We show that both chronic nicotine exposure starting during embryogenesis and continuing until adulthood (but not transient exposure limited to the perinatal period) and early life IAV infection separately are capable of driving lung dysfunction with age. Interestingly, limited differences in lung dysfunction were discovered with chronic exposure and early life IAV infection alone, whereas major lung dysfunctional differences were discovered for transient perinatal nicotine exposure followed by early life IAV infection suggesting a potentiation effect. Abnormalities in lung function were accredited to increased peri-airway collagen deposition and enlarged alveolar structures; the latter appeared due to alveolar simplification during development but also perhaps destruction during aging. We discovered that $\alpha 7 \mathrm{nAChRs}$ partially mediated these changes. All together, we found that indeed these early life exposures resulted in abnormalities in lung structure and function that persisted into adulthood. This model of nicotine exposure and early life IAV infection in young mice provides a novel tool for studying the impact of these exposures in lung. 


\section{TABLE OF CONTENTS}

HEADING $\quad$ PAGE

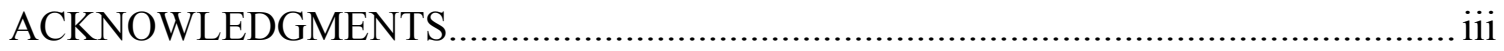

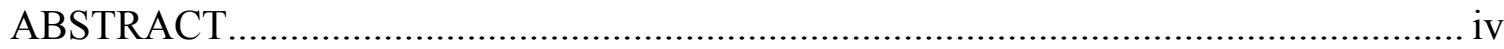

LIST OF TABLES ................................................................................................

LIST OF FIGURES ....................................................................................

CHAPTER I: INTRODUCTION.................................................................... 1

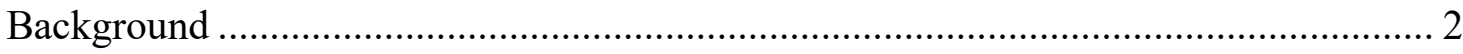

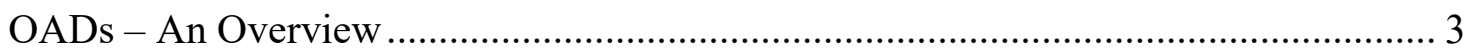

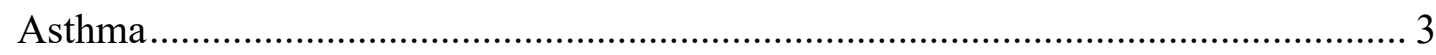

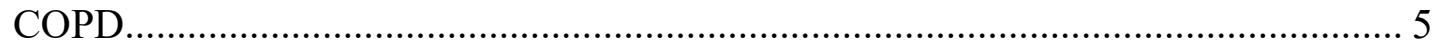

Early Life Events and Their Impact on OAD Development .................................... 7

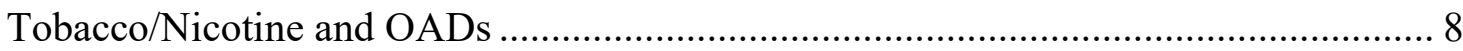

Respiratory Tract Infections Impact on OADs ............................................... 9

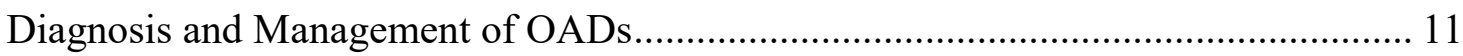

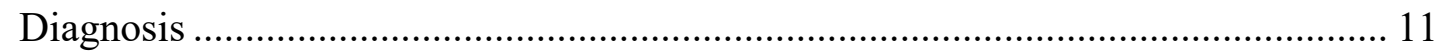

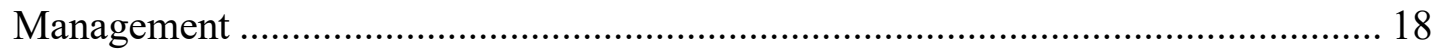

Animal Models and Methods to Study OADs ......................................................... 22

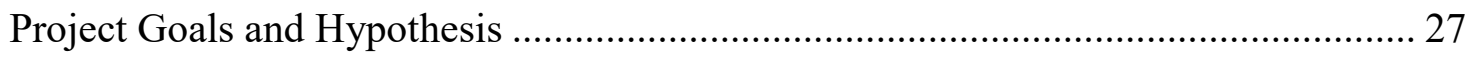


CHAPTER II: EARLY LIFE NICOTINE EXPOSURE LEADS TO STRUCTURAL AND

FUNCTIONAL ABNORMALITIES IN THE ADULT MURINE LUNG ..................... 30

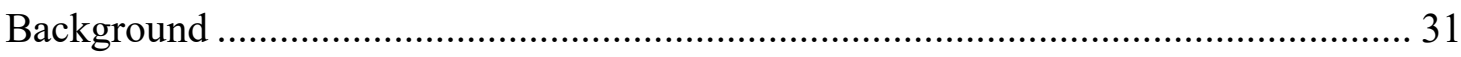

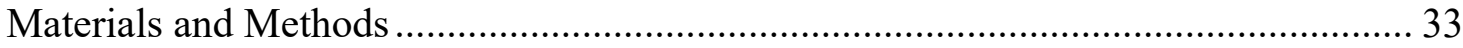

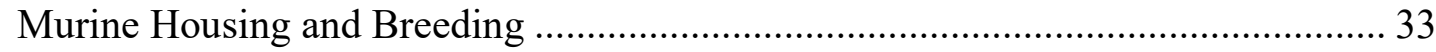

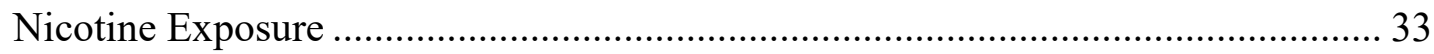

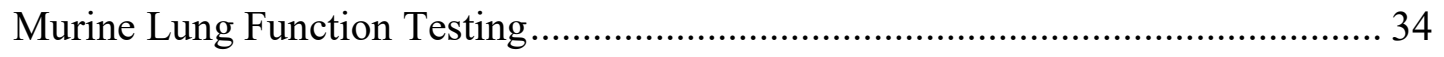

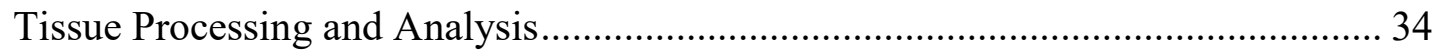

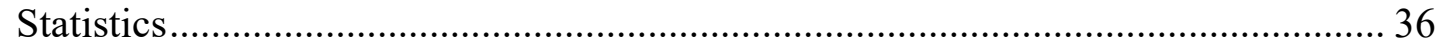

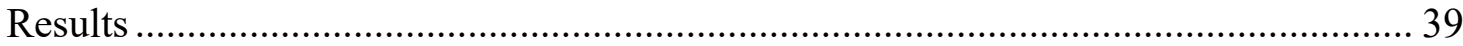

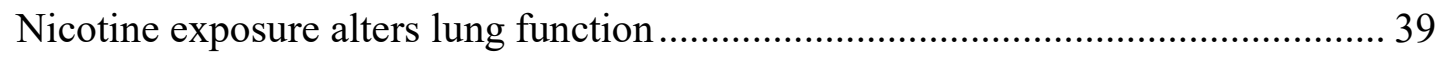

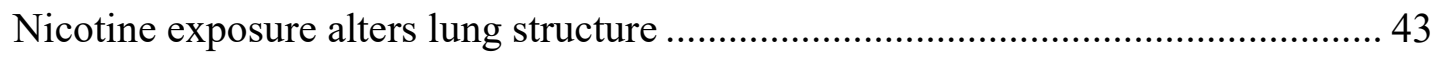

Nicotine exposure affects lung structure in the young lung ............................... 46

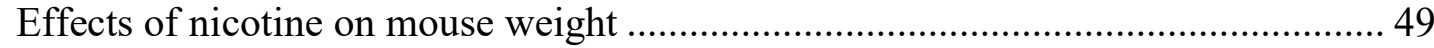

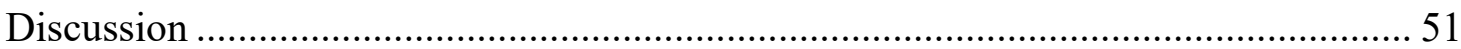

CHAPTER III: A MURINE MODEL FOR EARLY LIFE INFLUENZA A H1N1........ 59

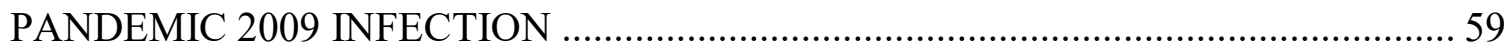

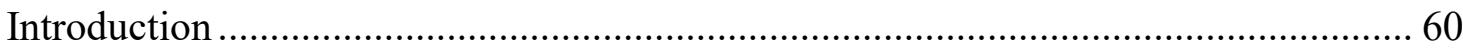

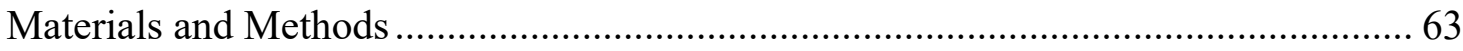

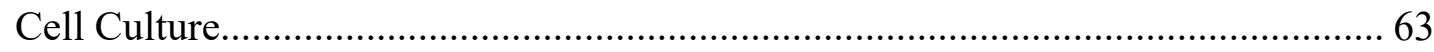

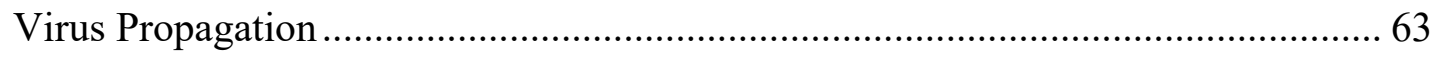


Viral Titer Determination 64

vRNA Isolation, cDNA Synthesis, and Hemagglutinin Sanger Sequencing ............ 66

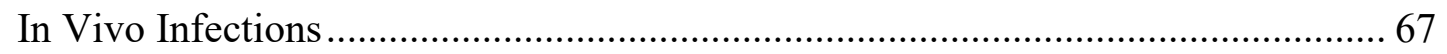

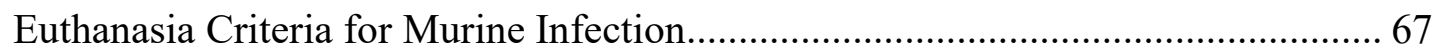

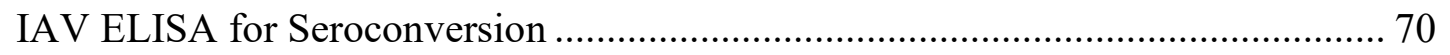

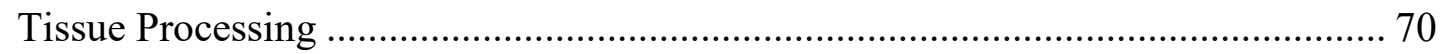

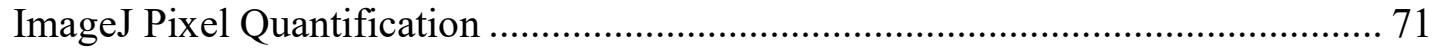

Lung Viral Load Quantitation ..................................................................... 72

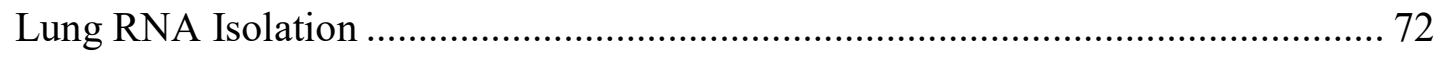

Primary Lung Fibroblast Isolation, Infection, and RNA Isolation ......................... 72

Transcriptome Assay (Clariom D Microarray) ................................................... 73

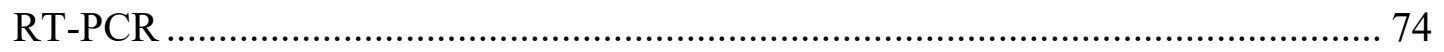

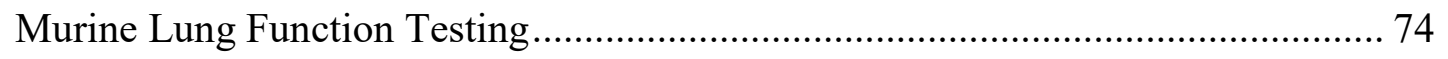

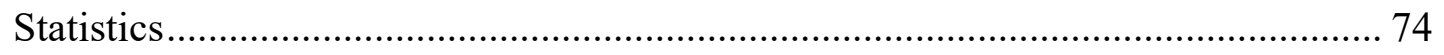

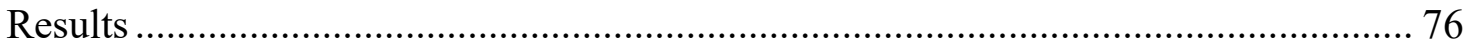

Propagation and Characterization of Viral Stock …............................................ 76

In vivo Replication Kinetics of Mice Infected at 14-Days of Age .......................... 78

In vivo Replication Kinetics of Mice Infected at 30-Days of Age .......................... 82

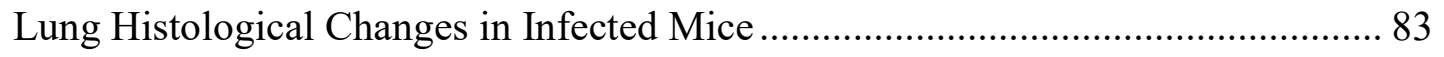

Whole Lung Global Transcript Profile in Infected Mice ..................................... 88 
Virus-Induced Tissue Remodeling Gene Expression in Primary Lung Fibroblasts.. 91

Pulmonary Function Testing .................................................................................. 94

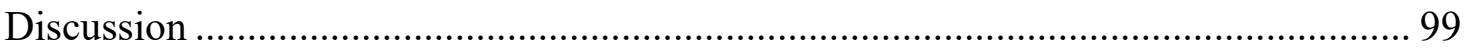

CHAPTER IV: NICOTINE EXPOSURE ACCOMPANIED WITH EARLY LIFE PANDEMIC H1N1 INFECTION LEADS TO MURINE ADULT LUNG

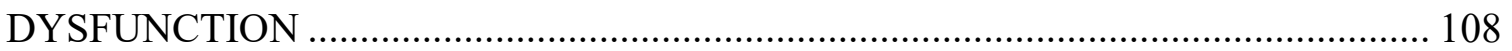

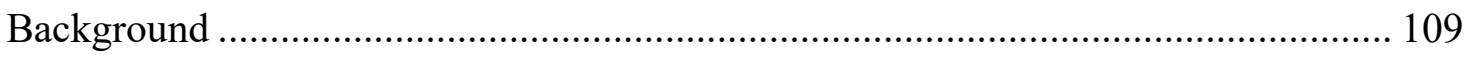

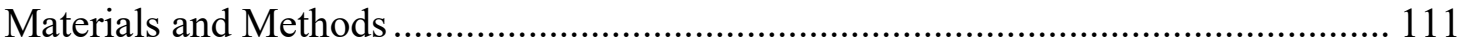

Murine Lung Function Testing ........................................................................... 111

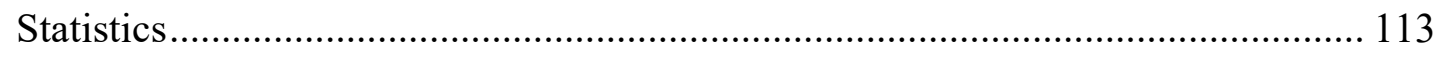

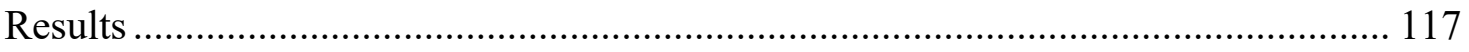

Growth Stunting After Infection with pH1N1 ...................................................... 117

Chronic Nicotine with or without pH1N1 Pulmonary Function Abnormalities ..... 119

Chronic Nicotine with pH1N1 Related Lung Structural Abnormalities .................. 123

Transient Nicotine Exposure with pH1N1 Infection-Related Pulmonary Abnormalities 125

Transient Nicotine and pH1N1 Related Lung Structural Abnormalities ................ 129

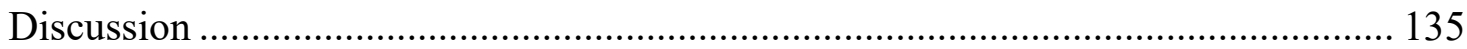


CHAPTER V: PULMONARY FUNCTIONAL ABNORMALITIES SEEN IN WILD TYPE C57BL/6 MICE WHEN EXPOSED TO NICOTINE WITH PH1N1 INFECTION IS ALLEVIATED IN A7 NACHR DEFICIENT MICE .......................................... 141

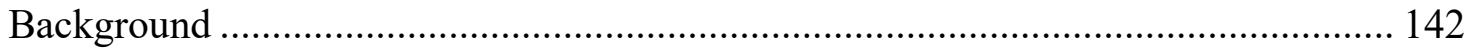

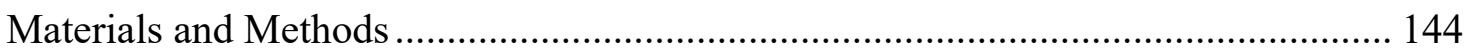

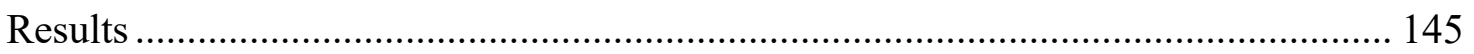

Transient Nicotine Exposure followed by pH1N1 infection in $\alpha 7 \mathrm{nAChR}$ Deficient

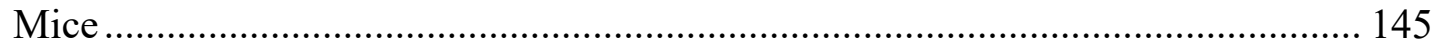

Lung Function in Nicotine Exposure followed by pH1N1 infection in WT and $\alpha 7$

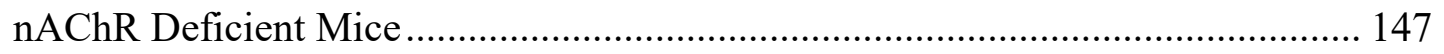

Lung Histology in Nicotine Exposure followed by pH1N1 infection in WT and $\alpha 7$

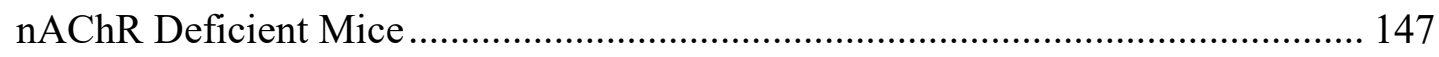

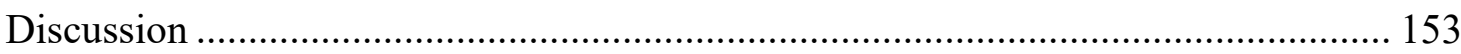

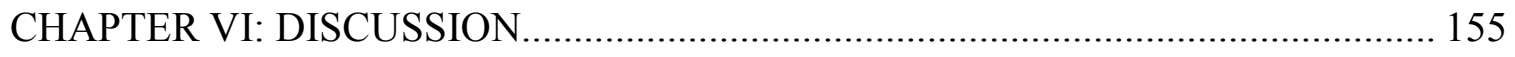

Strengths and Limitations of Analysis ......................................................... 159

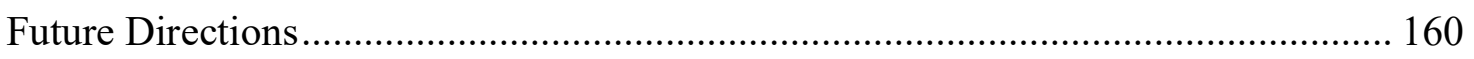

Different Viral Families and Different Age of Infection.................................... 160

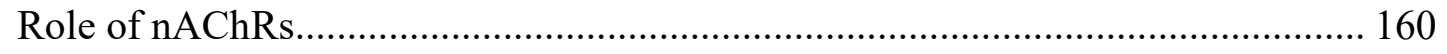

Immune Response Signaling and Implementing Immune Deficient Mice............. 161 


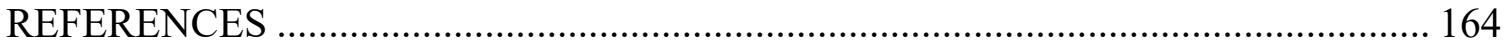

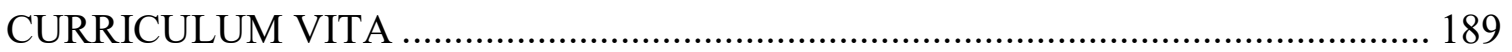




\section{LIST OF TABLES}

TABLE

PAGE

Table 1. Pulmonary Function Test Terms (Lung Capacities, Volumes, and Spirometry

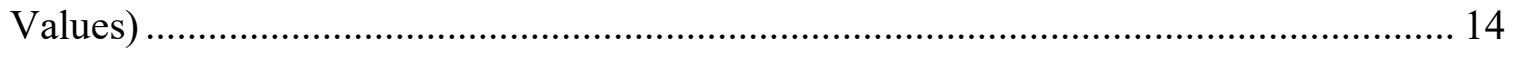

Table 2. Medications for Asthma and COPD ............................................................. 19

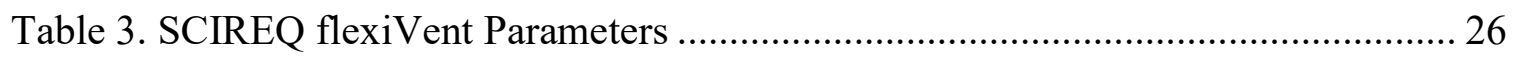

Table 4. Summary of Nicotine Pulmonary Function Testing ...................................... 42

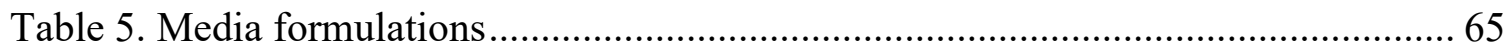

Table 6. In vivo Infections Humane Endpoint Criteria ............................................. 69

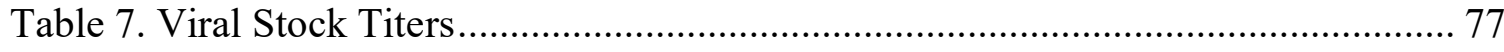

Table 8. Microarray Most Differentially Expressed Genes (pH1N1 vs. Uninfected) ...... 92

Table 9. Summary of pH1N1 Pulmonary Function Testing ........................................ 98

Table 10. Summary of Chronic Nicotine with pH1N1 Pulmonary Function Testing .... 122

Table 11. Summary of Transient Nicotine with pH1N1 Pulmonary Function Testing.. 128

Table 12. Pulmontary Fucntion Testing Summary …............................................ 158 


\section{LIST OF FIGURES}

FIGURE PAGE

Figure 1. Spirogram and Flow-Volume Curve from Pulmonary Function Tests ............ 15

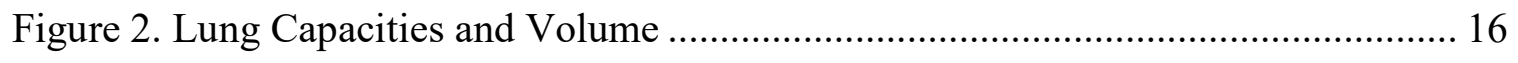

Figure 3. Obstructive Airway Disease Spirogram and Flow-Volume Curves................. 17

Figure 4. Potential Effects of Early Life Exposures on Lung Development ................... 29

Figure 5. Correlation of Pulmonary Function Test Outcome Parameters for Perinatal

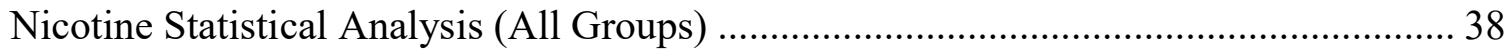

Figure 6. Perinatal Nicotine Exposure Experimental Design .................................... 40

Figure 7. Pulmonary Function Test Results for Nicotine Exposure at 210-Days of Age. 41

Figure 8. Lung Histology of Nicotine Exposed Mice at 210-Days of Age ..................... 44

Figure 9. Lung Histology of Chronic Nicotine Exposed Mice at 34-Days of Age ......... 47

Figure 10. Alveolar Septum Quantification Over Time in Chronic Nicotine Exposed Mice 48

Figure 11. Weights of Nicotine Exposed Mice Assessed for Lung Functional Abnormalities

Figure 12. Lung Viral Load, Survival, and Weight Change in 14 and 30-Day Old C57Bl/6

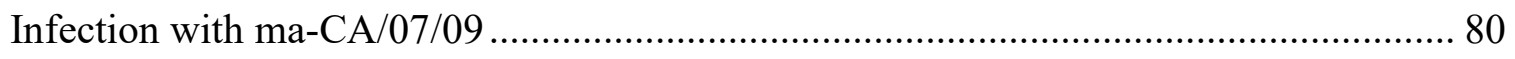

Figure 13. Lung Histology of ma-CA/07/09 Infected Mice at 34-Days of Age ............... 87 
Figure 14. Infection of 30-Day Old Mice with ma-CA/07/09 Microarray at 34-Days of Age 89

Figure 15. ma-CA/07/09 Infected Primary Lung Fibroblast Altered RT-PCR Tissue

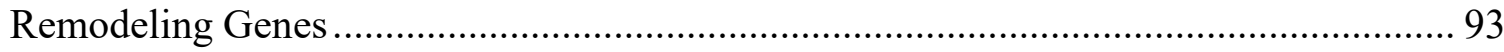

Figure 16. Correlation of Pulmonary Function Test Outcome Parameters for pH1N1

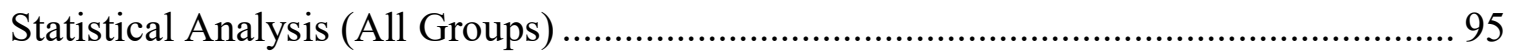

Figure 17. Pulmonary Function Test Results for ma-CA/07/09 Infection in 30-Day Old

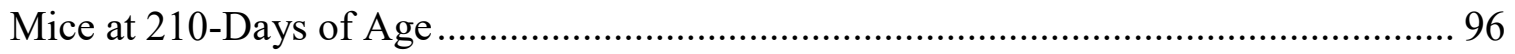

Figure 18. Perinatal Nicotine Exposure with pH1N1 Infection Experimental Design... 112 Figure 19. Correlation of Pulmonary Function Test Outcome Parameters for Transient Nicotine with pH1N1 Statistical Analysis (All Groups)......................................... 115 Figure 20. Correlation of Pulmonary Function Test Outcome Parameters for Chronic Nicotine with pH1N1 Statistical Analysis (All Groups).......................................... 116 Figure 21. Weights of Perinatal Nicotine Exposed Mice with pH1N1 Infection Assessed for Lung Functional Abnormalities .................................................................... 118 Figure 22. Pulmonary Function Test Results for Chronic Nicotine Exposure with pH1N1 Infection at 210-Days of Age 120

Figure 23. Lung Histology of Chronic Nicotine Exposure and pH1N1 Infection (Alone or in Combination) at 210-Days of Age 124 Figure 24. Pulmonary Function Test Results for Transient Nicotine Exposure with pH1N1 Infection at 210-Days of Age 126 Figure 25. Lung Histology of Transient Nicotine Exposure and pH1N1 Infection (Alone or in Combination) at 210-Days of Age...... 130 
Figure 26. Lung Histology of Transient Nicotine Exposure with pH1N1 Infection at 34Days of Age 131

Figure 27. Alveolar Septum Quantification Over Time in Transient Nicotine Exposure with pH1N1 Infection 132

Figure 28. Histological Signs of Viral Related Pneumonitis in Transient Nicotine with pH1N1 at 210 Days of Age. 134

Figure 29. Weights of Transient Nicotine Exposed Mice with pH1N1 Infection (Wild Type and $\alpha 7 \mathrm{nAChR}$ Deficient) Assessed for Lung Functional Abnormalities 146 Figure 30. Pulmonary Function Test Results for Transient Nicotine with pH1N1 Infection in $\alpha 7 \mathrm{nAChR}$ Deficient Mice 149

Figure 31. Lung Histology of Transient Nicotine Exposure with pH1N1 Infection at 210Days of Age for $\alpha 7$ AChR Deficient Mice 150

Figure 32. Histological Signs of Viral Related Pneumonia in Transient Perinatal Nicotine Not Seen in $\alpha 7 \mathrm{nAChR}$ Deficient Mice 152 
CHAPTER I: INTRODUCTION 


\section{$\underline{\text { Background }}$}

Chronic Obstructive Airway Diseases (OADs) are respiratory diseases that affect millions of people worldwide, and are characterized by chronic inflammation and tissue remodeling in the lung (1-3). Asthma and Chronic Obstructive Pulmonary Disease (COPD) are two highly visible examples of OADs $(1,3,4)$. The onset of asthma occurs typically in adolescence years, whereas COPD onsets around 40 years of age (2-4). While asthma can improve or progress after childhood, bronchospasms can be ameliorated with medication (5). Asthma is characterized by airway inflammation, airflow obstruction (via airway wall inflammation and remodeling), and airway hyperreactivity (AHR), which is when certain factors (allergens, cold air, etc.) cause exaggerated narrowing of the airways (2-4). In 2015, asthma was found to be the most prevalent chronic respiratory disease, affecting approximately 358 million individuals globally, and with the prevalence expected to increase (6). COPD is an irreversible, progressive disease, which is diagnosed in people with chronic bronchitis, emphysema, or a combination of the two (2-4). In 2015, COPD was diagnosed in 174 million people globally, with prevalence and mortality increasing greatly with aging (7).

Worldwide, chronic respiratory diseases represent a leading cause of mortality and morbidity, with asthma and COPD being the most common (7). Annually, asthma and COPD result in over $\$ 100$ billion in direct and indirect costs in the U.S., and over $\$ 80$ billion in the European Union (8-10). Importantly, the majority of cost associated with these diseases is due to indirect costs related to loss of workplace productivity $(7,8)$. It is estimated that $40 \%$ of the annual healthcare cost could be avoided by preventing 
complications and hospitalizations related to these conditions through the development of effective treatments $(7,8)$.

An emerging concept relates how early life exposures may promote the development of OADs later in life. We hypothesize that perinatal events lead to adaptations (or maladaptations) that promote alterations in lung structure and function that are long-lasting and promote lung disease in the adult. In Chapter II, we report findings regarding the impact of perinatal nicotine exposure on lung structure and function. In Chapter III, we investigate a new murine model for early life influenza infection which triggers lung tissue remodeling. In Chapter IV, we combine the two exposures to determine if they potentiate each other. In Chapter $\mathrm{V}$, we assess the role of cholinergic signaling in mediating some of these effects by testing genetically modified animals lacking a specific nicotinic acetylcholine receptor (nAChR). Before this, we present an overview of OADs and their development and how early life events may promote the development of OADS. We explain how OADs are diagnosed and managed, and we describe how animal models are capable of providing insight about their pathophysiology.

\section{$\underline{\text { OADs - An Overview }}$}

\section{Asthma}

Asthma is a condition characterized by airway inflammation and airflow limitation due to bronchoconstriction (resulting in wheezing) after a stimulus (6). The risk factors for the development of asthma are, but not limited to, both environmental exposures and genetics (11-13). Asthma is known to be influenced by geographical location (developing countries having higher prevalence) where environmental factors play a main role in the 
development of the disease $(12,14,15)$. There are a number of correlations between exposure to environmental factors, asthma development, and exacerbations, with poor air quality (gaseous pollutants sulfur dioxide and nitrogen dioxide), diesel exhaust (polycyclic aromatic hydrocarbons, carbon monoxide, and nitric oxide), animal dander and excreta, dust, dust mites, genetically modified food, fungus, mold, and most importantly, environmental tobacco smoke (ETS) (16).

Genetics are also involved and although the inheritance of asthma does not follow classical Mendelian patterns, there is clear evidence linking asthma development to genetics $(12,13)$. The first studies involving asthma and genetic factors were published in the early 1900's; they suggested that inheritance of the disease was predicted to be autosomal dominant (12). A twin study conducted in the 1970's as well as an Australian study in the 1990's showed that the monozygotic (MZ) concordance rate was larger than the dizygotic (DZ) concordance rate ( 4-fold and $\sim 3$-fold), where heritability for the latter was higher for males than females $(12,13,17)$. A higher concordance rate in MZ twins than in (DZ) twins is known to be evidence for a genetic component being a contributing factor (18). Many other studies looking at heritability of asthma found similar findings $(19-23)$.

Airway remodeling is a key aspect of asthma that results in loss of lung function (24). Airway remodeling includes epithelial cell hypoplasia or denudation with goblet cell metaplasia, airway smooth muscle hypertrophy, angiogenesis, and most importantly, increased deposition of extracellular matrix (ECM) components such as collagen I and III (24-26). In this and other chronic lung conditions, ECM components are found to be increased in all lung compartments (central airways, airway smooth muscle, parenchyma, 
and vessels) (27). Thickening of the airway was the first structural alteration described in asthma $(25,26)$. Although thickening of the airway may have a negligible effect on baseline airway resistance (defined below), it has a profound effect on narrowing of airways after a stimulus (26).

The lung ECM controls resistance or elasticity of the lung structure, which ultimately effects lung volume (24). The cells that play a predominant role in deposition of ECM components are primarily fibroblasts, via SMAD signaling pathways triggered from epithelial cell TGF $\beta$ production, among other pathways (24). Increased TGF $\beta$ production arises in response to damage that occurs from $\mathrm{T}$ helper $2\left(\mathrm{~T}_{\mathrm{H}} 2\right)$ cell driven eosinophilic inflammation (24). Inflammatory cells (which reside in close proximity to fibroblasts) are recruited to the airway in response to inhaled allergens causing epithelial destruction and desquamation $(24,26)$. Furthermore, airway inflammation and resulting remodeling processes may be exacerbated through respiratory viral infections such as influenza (26). Lastly, in utero fetal programming through maternal smoking may create a gestational environment that drives $\mathrm{T}_{\mathrm{H}} 2$ differentiation and thus, lead to early life asthma in the offspring (26).

COPD

COPD is an umbrella term that includes chronic bronchitis, emphysema and chronic bronchiolitis. Risk factors for the development of COPD are from both environmental exposures and genetic factors. Smoking is the leading cause of COPD globally (causing $85-90 \%$ of cases) in developed countries, and smokers are 13 times more likely to develop and die from COPD compared to non-smokers (28-30). Another factor 
influencing the development of COPD, especially emphysema, is deficiency of $\alpha-1$ antitrypsin, an inherited autosomal codominant genetic disorder characterized by low levels of this protease $(31,32)$. Deficiency of $\alpha-1$ antitrypsin is found in less than $1 \%$ of COPD patients, although $5 \%$ of individuals diagnosed with COPD are thought to have this deficiency (33). Another factor for the development of COPD is aging (34).

Chronic bronchitis/bronchiolitis is characterized by narrowing of the airways by inflammation, epithelial cell hypoplasia, excessive mucus production, and excess ECM protein synthesis (mainly collagens and fibronectin). This leads the airways to becoming narrower, with decreased airflow. Chronic inflammation of the peripheral small airways (bronchioles) is a key characteristic of chronic bronchiolitis which may arise through extrinsic factors binding Toll-like receptors (TLR) and thus induction of the innate immune response (35). Most importantly, the upregulation of transcription factor NFKB also occurs due to this TLR signaling (35). These events cause excessive immune cell (macrophages and neutrophils) infiltration, resulting in inflammation of the airways and mucus hypersecretion, which causes narrowing of the airways thereby limiting airflow (35).

On the other hand, emphysema results from parenchymal destruction, predominantly in areas distal to the bronchi and bronchiole where alveoli reside. This destruction causes decreased elastic recoil that impacts exhalation. Lack of $\alpha-1$ antitrypsin causes decreased protection against neutrophil elastase, resulting in destruction of alveolar walls and resulting in increased alveolar volume (32). Additionally, TLR induced neutrophil infiltration increases matrix metalloproteinase (MMP) production as well as decreases tissue inhibitors of MMP (TIMPs) further enhancing proteolytic activity (35). Increased neutrophil infiltration results in increased neutrophil elastase secretion (36). 
MMPs are also responsible for the destruction of the lung ECM, activation of TGF $\beta$, and increased cytokine signaling (37). ECM destruction, especially elastin, causes decreased elastic recoil, which will greatly effect lung volumes and hyperinflation (38). Increased TGF $\beta$ causes increased ECM deposition through SMAD signaling pathways ultimately causing increased collagen and fibronectin production $(39,40)$. Lastly, it is well established that in the lungs of humans with COPD or asthma (and in murine models), IL$1 \beta$ is upregulated, promoting inflammation ultimately resulting in distal airway enlargement and production of MMP 9/12 (41).

\section{Early Life Events and Their Impact on OAD Development}

In general, OADs are believed to result from environmental exposures in a susceptible genetic background. More recently, evidence has emerged suggesting that the development of OADs in adulthood or adolescence is greatly influenced and perhaps predetermined by events during embryogenesis or early life (42). In other words, lung structural and functional changes in the young mammals represent a major susceptibility factor for development of OAD later in life. Stern and colleagues published in 2007 that lung function decreases with age (43). This suggests that it is not a question of "if" a person's lung function will deteriorate, but at what speed will it deteriorate. In some cases, this deterioration is accelerated to the point of developing OADs $(43,44)$. Therefore, if a person has low lung function early in life, they will have an increased likelihood for reaching lower lung function to the point of developing OADs later in life unless therapeutic management, environmental changes, and/or lifestyle interventions takes place. Of the entire population diagnosed with asthma, $9.3 \%$ are children and $8 \%$ are adults, 
proving that asthma is not only a childhood disorder but also affects individuals throughout adulthood (45). However, children who suffer from severe persistent asthma are 32 times more likely to develop COPD as adults compared to non-asthmatic children (45). This suggests a correlation between the development of lower lung function in childhood (e.g. asthma) and pulmonary abnormalities persisting into adulthood and promoting the development of more serious respiratory defects in adulthood.

\section{$\underline{\text { Tobacco/Nicotine and OADs }}$}

There is an abundant amount of epidemiological data linking ETS to OADs and the development of asthma in children $(4,46,47)$. Smoking during pregnancy or more specifically fetal nicotine exposure, has been shown to result in the offspring to have decreased lung function in childhood, which subsequently causes lifelong lung functional abnormalities $(43,48-52)$. In the United states, it is estimated that approximately $10 \%$ of pregnant mothers smoke cigarettes at some point during their pregnancy, and $50 \%$ of mothers who are smokers continue to use cigarettes throughout pregnancy $(48,53)$. In 2016, Kentucky and West Virginia were determined to have the highest prevalence of maternal smoking (at approximately 18 and 25\%) (53). In addition, postnatal exposure to ETS remains widespread (54). A cross sectional study of data sets generated worldwide found that children exposed to ETS show decreased lung function (55). Despite this, it is estimated that $40 \%$ of children worldwide are still exposed to ETS (54).

ETS contains over 7,000 components, one of which is nicotine, which is responsible for the addictive behavior of smoking $(56,57)$. We are interested in the effects of nicotine, which acts via specific cell surface receptors capable of intracellular signaling. There are 
two types of acetylcholine receptors (AChR), nicotinic (ion gated) and muscarinic (gcoupled) (58-60). The primary agonist for muscarinic AChR is muscarine, and acetylcholine or nicotine are the primary agonists that bind to nicotinic AChR (nAChR) (58-60). Nicotine, as well as its metabolite, cotinine, pass freely through the placenta achieving concentrations in the fetus greater than that of the mother (61). Our laboratory, in collaboration with Elliot Spindel, has previously reported that perinatal nicotine (acting through the $\alpha 7 \mathrm{nAChR}$ ) induced increased lung branching morphogenesis, increased collagen deposition in the lung, and increased responsiveness to methacholine (an M3 muscarinic receptor agonist) (42). Similar findings to ours have been published in nonhuman primate models that showed that perinatal nicotine not only decreased lung function and caused alveolar hypoplasia, but also increased the expression of $\alpha 7 \mathrm{nAChRs}$ and collagen deposition around large airways and vessels (42, 62-64). These studies suggest that animal models can mimic the effects of smoking (nicotine) in humans and could be used to further our limited understanding of the mechanisms driving the development of OADs. We believe nicotine is the constituent of ETS that is driving changes in human offspring lung function due to its ability to reach the fetus during gestation.

\section{$\underline{\text { Respiratory Tract Infections Impact on OADs }}$}

In addition to the offspring of smokers (perinatal nicotine exposure) and current tobacco smoke users being more susceptible to OADs, there is also evidence for lower respiratory infections, such as but not limited to influenza H1N1, to lead to the development of OADs and causing an exacerbated disease state $(65,66)$. Viral lower respiratory infections during childhood induce airway inflammation, which may explain 
the association with OADs $(46,67-70)$. Children younger than two years old (when the lung is still in development) are more prone to serious influenza complications than any other age group in the United States, and the risk is heightened when asthma is present (71). Furthermore, influenza alone has been shown to increase AHR in response to methacholine, a bronchoconstrictor used by investigators to unveil AHR and other lung functional abnormalities $(72,73)$.

Influenza infections have been extensively demonstrated to induce a robust, diverse, complicated innate and adaptive immune response to clear the infection (74-76). Briefly, the primary target of influenza are epithelial cells (77). Infection of the epithelium causes the release of proinflammatory cytokines and chemokines, most notably IL-1 $\beta$ and CCL2 (74). These signaling events cause fibroblast and Treg production of TGF $\beta$, which is activated through influenza neuraminidase $(74,78)$. Furthermore, IL-1 $\beta$ is also known to cause macrophages to increase MMP production (37). These events could potentiate the develop of OADs as discussed above.

When infection begins in the epithelium, influenza is recognized by pattern recognition receptors (TLR, RIG-I, and NLRP3), which leads to activation and secretion of type I interferons (IFN $\alpha$ from macrophages, pneumocytes, and dendric cells), inducing an antiviral state (most notably the NFkB pathway), leading to production of pro- and antiinflammatory cytokines and chemokines (76). Alveolar macrophages are the first cells that encounter the virus and produce chemokines to recruit neutrophils, monocytes, and natural killer T (NKT) cells to the infected airway regions, in which NKT cells target infected epithelial cells for viral clearance $(76,79)$. Monocytes and neutrophils clear the respiratory system of infected dead cells (76). The influx of neutrophils could potentially increase 
neutrophil elastase secretion and, therefore, promote emphysema. If this initial innate response does not successfully clear the infection, released cytokines instruct the response of the adaptive immune system for a continued battle to rid the body of infection (76). The bridge between the innate and adaptive immune responses are the antigen presenting dendritic cells (MHCII) for naïve $\mathrm{CD}^{+}$generation and NKT or dendritic cells for $\mathrm{CD}^{+} \mathrm{T}$ cell (MHCI) generation $(74,75)$. Differentiation into T cells occurs through the type of cytokine secretion occurring by antigen presenting cells (80). Briefly, $\mathrm{CD}^{+} \mathrm{T}$ cells recognize and eliminate influenza infected cells through release of perforin and granzymes (75), whereas, $\mathrm{CD}^{+} \mathrm{T}$ cells promote $\mathrm{B}$ cell responses $\left(\mathrm{T}_{\mathrm{H}} 2\right.$ and regulatory $\mathrm{T}$ cells (Treg)), promote proinflammatory (IFN $\gamma$ and IL-2) immune responses $\left(\mathrm{T}_{\mathrm{H}} 1\right)$, and regulate proinflammatory immune response ( $\mathrm{T}_{\mathrm{H}} 17$ and $\left.\mathrm{Treg}\right)(75)$.

Lastly, TGF $\beta$ has been published to be produced from murine epithelial cells and Treg cells after influenza infection $(74,81)$. The production of epithelial TGF $\beta$ in mice has been demonstrated to increase viral burden and pathology via suppressing IFN and consequently $\mathrm{T}_{\mathrm{H}} 1 / 2$ cells production $(81,82)$. Furthermore, the production of TGF $\beta$ promotes differentiation of Treg cells, which causes Treg cells to produce additional TGF $\beta$ (83). This synergized TGF $\beta$ production may also cause increased tissue remodeling as mentioned before.

\section{Diagnosis and Management of OADs}

\section{Diagnosis}

For physicians, the diagnosis of OAD begins with a question and observation-based assessment. This assessment addresses the patients history of respiratory symptoms such 
as chest pain, cough, orthopnea, wheezing, cyanosis, abnormal blood gases, shortness of breath or dyspnea, increased sputum production, etc. (84). After consulting and examining the patient, the physician may then have the patient undergo imaging tests such as a radiograph or computerized tomography (CT) scan followed by a physical quantitative assessment of lung function via spirometry. Using imaging tests for the diagnosis of OADs is limited, although they are useful for a physician to infer what type of disease is occurring in order to begin treatment, while the patient waits for spirometry testing $(85,86)$. For COPD, the physician would be looking to find multiple lucencies indicating air trapping, bronchial thickening (chronic bronchiolitis), hyperinflation, and gross lung destruction (emphysema) $(85,86)$. Whereas, in asthmatics there may not be tissue destruction or lucencies, but hyperinflation is often evident (87).

Pulmonary function tests (PFT), using spirometry, is the key diagnostic tool used by clinicians to diagnose OADs in humans (88). Spirometry can be conducted using a spirometer in which a person breathes in and out of a tube (to determine airflow). A plethysmograph, in which the person breathes inside a sealed chamber, is used to determine lung volume. Spirometry allows quantification of key pulmonary functional characteristics, which depict volume of the lung as well as airflow to and from the lung (Table 1). From pulmonary function tests result a volume versus time (spirogram) (Fig. 1A), a flow (volume over time) versus volume curve (Fig. 1B), and a lung capacity/volume graph (Fig. 2), for physicians to diagnose a disease state. When determining an abnormality, a bronchodilator challenge test could then implemented to dissect whether the patient has reversible airflow limitation; the bronchodilator will alleviate abnormalities seen for asthma, but typically not for COPD $(6,89)$. When diagnosing a OAD, the first 
parameter used to determine a diseased state is the ratio of FEV1 and FVC (Table 1) (89, 90). A FEV1/FVC ratio less than 0.7, with an normal or decreased FEV1 indicates an OAD $(89,90)$. Other key changes in functional parameters observed for OADs, compared to normal subjects, are decreased peak expiratory flow rates (Fig. 3). 
Table 1. Pulmonary Function Test Terms (Lung Capacities, Volumes, and Spirometry Values)

\begin{tabular}{|ccl|}
\hline Term & Abbreviation & \multicolumn{1}{c|}{ Definition } \\
\hline Forced Vital Capacity & FVC & $\begin{array}{l}\text { The total volume of air expired after maximally forcing } \\
\text { expiration of air after maximum inhalation }(89,90)\end{array}$ \\
\hline $\begin{array}{c}\text { Forced Expiratory } \\
\text { Volume at 1 second }\end{array}$ & FEV1 & $\begin{array}{l}\text { The FVC at one second after starting expiration after } \\
\text { maximum inhalation }(89,90)\end{array}$ \\
\hline $\begin{array}{c}\text { Peak Expiratory Flow } \\
\text { Rate }\end{array}$ & PEFR & $\begin{array}{l}\text { The maximum flow of air achieved when subject is } \\
\text { exerting maximum exhalation after maximum inhalation } \\
(89,90)\end{array}$ \\
\hline $\begin{array}{c}\text { Peak Inspiratory Flow } \\
\text { Rate }\end{array}$ & PIFR & $\begin{array}{l}\text { The maximum flow of air achieved when subject is } \\
\text { exerting maximum inhalation after maximum inhalation } \\
(89,90)\end{array}$ \\
\hline Total Lung Capacity & TLC & $\begin{array}{l}\text { The volume of air in lung at maximum inflation after } \\
\text { maximum inhalation }(89,90)\end{array}$ \\
\hline Inspiratory Capacity & IC & $\begin{array}{l}\text { The maximum volume of air that can be inhaled from } \\
\text { the resting expiratory level }(89,90)\end{array}$ \\
\hline $\begin{array}{c}\text { Functional Residual } \\
\text { Capacity }\end{array}$ & FRC & $\begin{array}{l}\text { The volume of air in the lungs at resting end expiration } \\
(89,90)\end{array}$ \\
\hline $\begin{array}{c}\text { Inspiratory Reserve } \\
\text { Volume }\end{array}$ & IRV & $\begin{array}{l}\text { The maximum volume of air inhaled after the normal } \\
\text { inhalation level }(89,90)\end{array}$ \\
\hline Tidal Volume & TV & $\begin{array}{l}\text { The volume of air between the normal inhalation and } \\
\text { exhalation levels, or the volume of air inhaled or exhaled } \\
\text { at resting state }(89,90)\end{array}$ \\
\hline $\begin{array}{c}\text { Expiratory Reserve } \\
\text { Volume }\end{array}$ & ERV & $\begin{array}{l}\text { The maximum volume of air exhaled after the normal } \\
\text { exhalation volume }(89,90)\end{array}$ \\
\hline Residual Volume & RV & $\begin{array}{l}\text { The volume of air remaining in the lung after the } \\
\text { maximum exhalation volume }(89,90)\end{array}$ \\
\hline
\end{tabular}


A

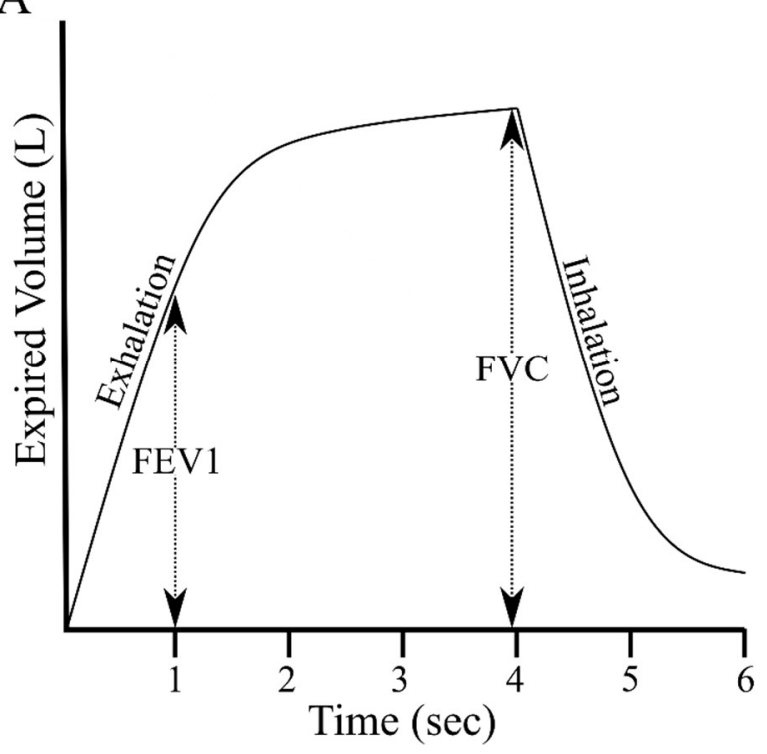

B

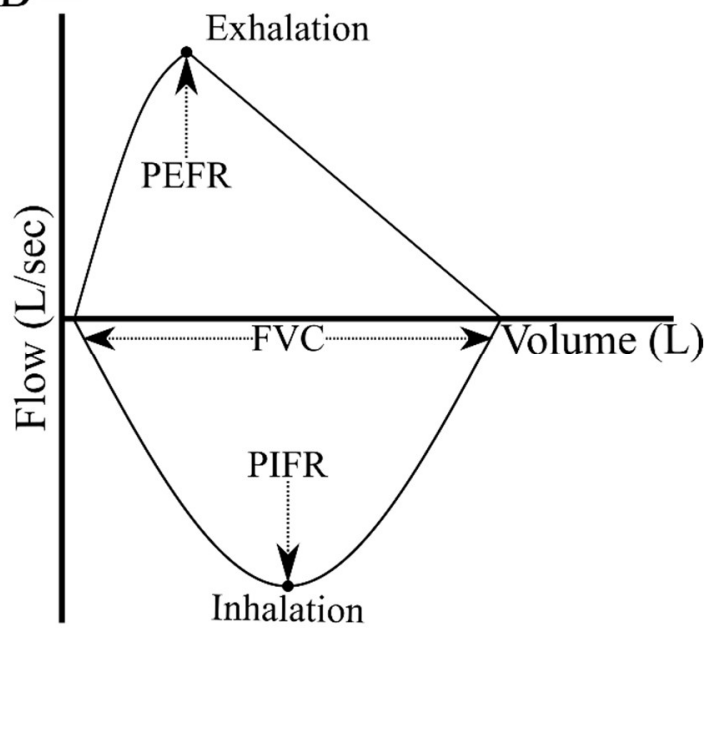

Figure 1. Spirogram and Flow-Volume Curve from Pulmonary Function Tests

Representative Graphs from PFT in humans. Spirogram with volume of air represented on $\mathrm{x}$-axis and time after exhalation or inhalation on the y-axis (A). Flow-Volume Curve with volume represented on x-axis and flow represented on y-axis (B). Adapted from Miller et al. and Quanjer et al. (91, 92). 


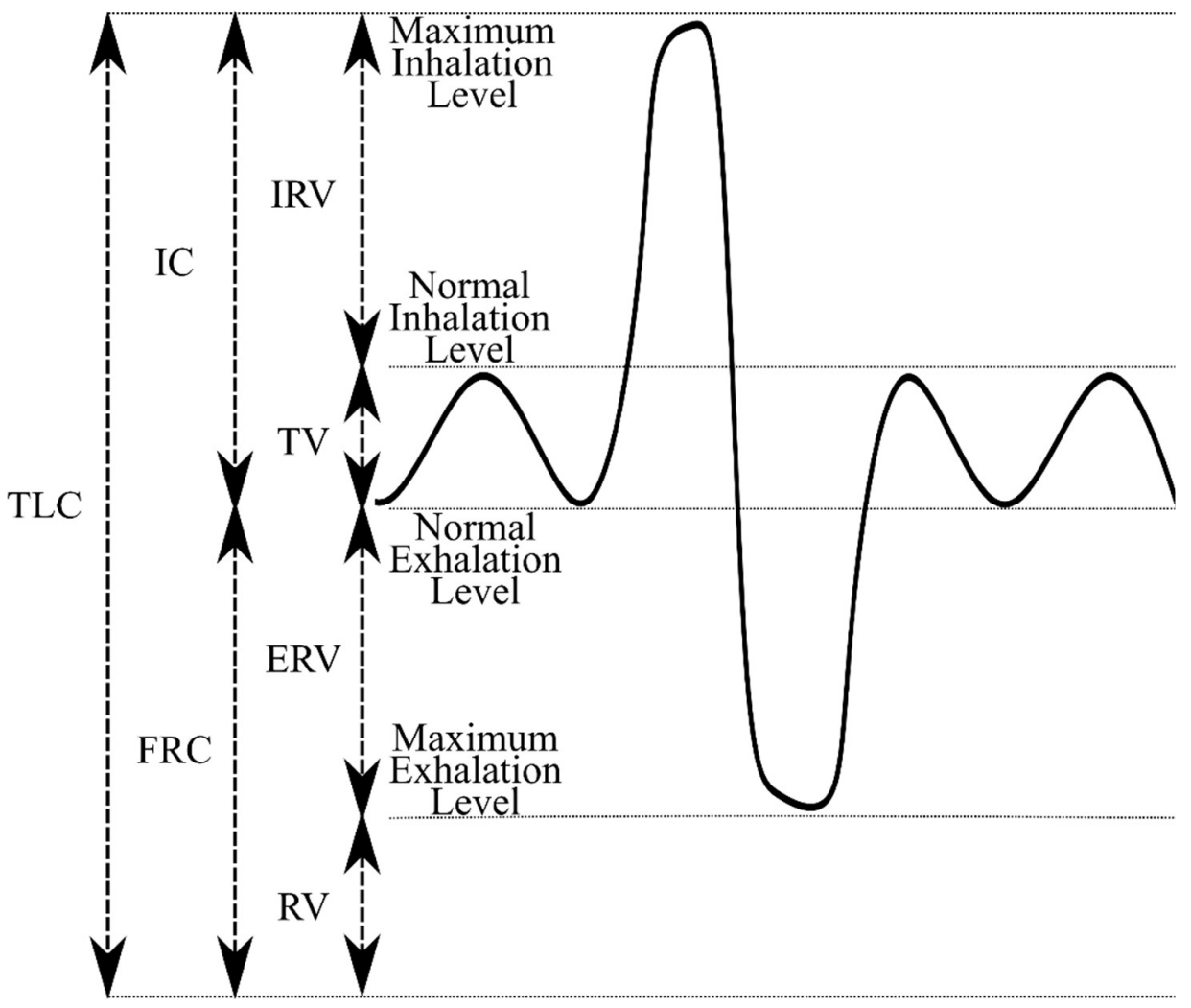

Figure 2. Lung Capacities and Volume

Curve represent the normal breathing pattern exhibited in human subjects when having pulmonary function tests conducted. Adapted from Wanger et al. and Miller et al. $(91,93)$. 
A

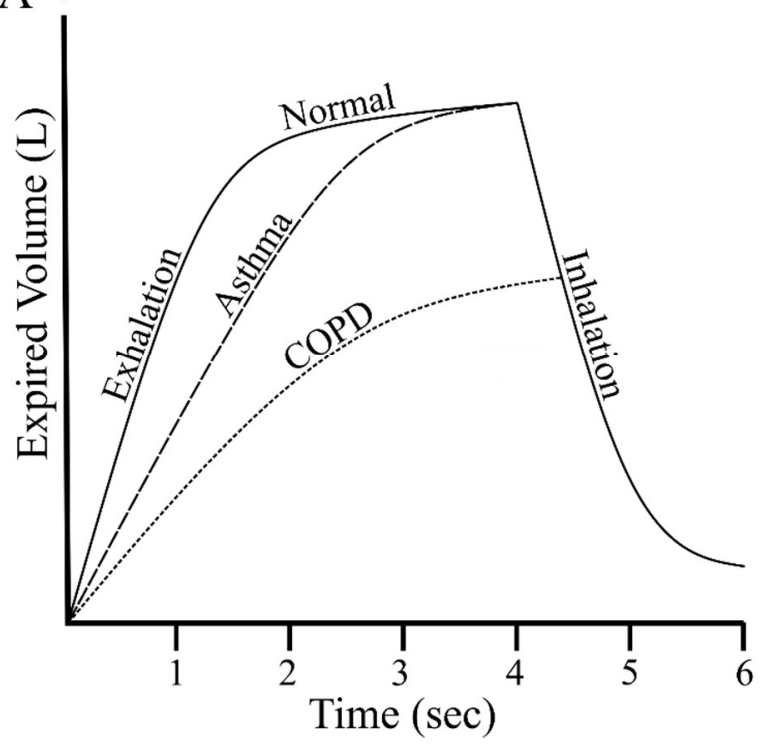

B

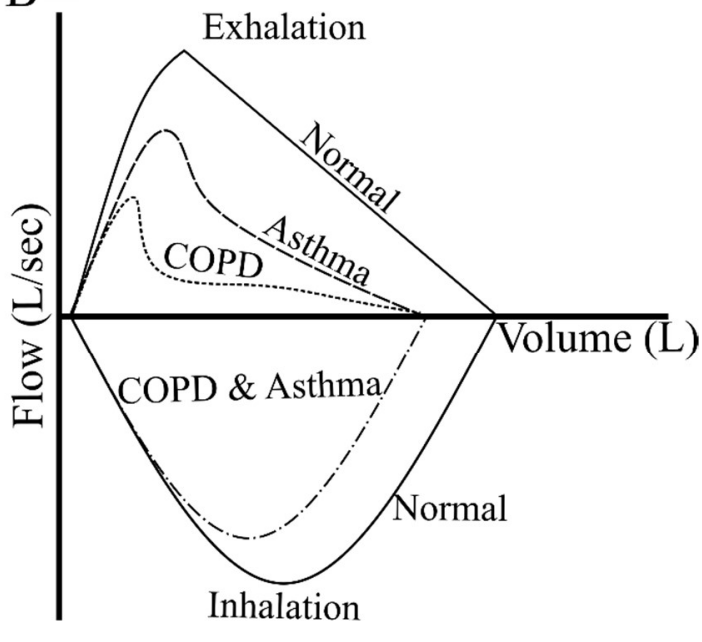

Figure 3. Obstructive Airway Disease Spirogram and Flow-Volume Curves

Representative spirogram (A) and flow-volume curve (B) of normal subjects compared to asthmatics and COPD patients compared to healthy people. Adapted from Miller et al. and Quanjer et al. (91, 92). 


\section{Management}

Decreased lung function due to COPD is permanent, irreversible and progressive whereas with asthmatics bronchoconstriction is known to be reversible through the administration of corticosteroids and/or long-acting beta agonists. For asthma, focus is on management of the disease to prevent exacerbations as opposed to resolving the condition (6). There are three forms of treatment for asthma, which are quick relief, long term control, and non-pharmacological treatments $(94,95)$.

Therapeutics commonly used for quick relief include short acting beta 2 agonists (SABAs), systemic corticosteroids (SCSs), and short acting muscarinic antagonists (SAMA) (94). SABAs are delivered through aerosol and are the fastest therapy for alleviating bronchoconstriction, via cAMP production leading to airway smooth muscle relaxation (Table 2) (94). SABAs and SCSs are the first suggested treatment for asthma. SCSs can be delivered intravenously or orally with similar efficacy (Table 2) $(96,97)$. Corticosteroids act in many ways to relieve asthma including enhancing the betaadrenergic responses (to relieve muscle spasm), reversing mucosal edema, decreasing vascular permeability (by vasoconstriction), and inhibiting the release of leukotriene C4 and D4 (98). Lastly, SAMAs are another form of quick relief therapy for asthma, although SAMAs are not as quick as SABAs, taking 20-30 minutes for their effects to take place

(Table 2) (94). SAMAs work as bronchodilators by blocking acetylcholine leading to bronchial smooth muscle relaxation (94). 
Table 2. Medications for Asthma and COPD

\begin{tabular}{|c|c|c|}
\hline Disease & Therapeutic Class & Generic Pharmacological Name \\
\hline \multirow[t]{11}{*}{ Asthma } & \multicolumn{2}{|l|}{ Ouick Relief } \\
\hline & $\begin{array}{l}\text { Short Acting Beta } 2 \\
\text { Agonists }\end{array}$ & Albuterol and Terbutaline \\
\hline & $\begin{array}{l}\text { Systemic } \\
\text { Corticosteroids }\end{array}$ & $\begin{array}{l}\text { Orally: Methylprednisolone, Prednisolone, and Prednisone } \\
\text { Intravenously: Methylprednisolone and Triamcinolone }\end{array}$ \\
\hline & SAMAs & Ipratropium \\
\hline & \multicolumn{2}{|l|}{ Long Term Control } \\
\hline & $\begin{array}{l}\text { Inhaled } \\
\text { Corticosteroids }\end{array}$ & $\begin{array}{l}\text { Budesonide, Fluticasone, Mometasone, Beclomethasone, } \\
\text { Ciclesonide, and Triamcinolone }\end{array}$ \\
\hline & $\begin{array}{l}\text { Long Acting Beta } 2 \\
\text { Agonists }\end{array}$ & Salmeterol, Formoterol, Arformoterol, and Vilanterol \\
\hline & $\begin{array}{l}\text { Inhaled } \\
\text { Corticosteroids }+ \\
\text { Long Acting Beta } 2 \\
\text { Agonists }\end{array}$ & $\begin{array}{l}\text { Budesonide + Formoterol } \\
\text { Fluticasone + Salmeterol } \\
\text { Fluticasone + Vilanterol } \\
\text { Mometasone + Formoterol }\end{array}$ \\
\hline & $\begin{array}{l}\text { Leukotriene } \\
\text { Modifiers }\end{array}$ & $\begin{array}{l}\text { 5-lipoxygenase Inhibitors: zileuton } \\
\text { Cysteinyl Leukotreine-1 Receptor Antagonists: } \\
\text { Montelukast, Zafirlukast, and Pranlukast }\end{array}$ \\
\hline & Anti-IgE Therapy & Omalizumab \\
\hline & $\begin{array}{l}\text { Systemic } \\
\text { Corticosteroids }\end{array}$ & Methylprednisolone, Prednisolone, and Prednisone \\
\hline \multirow[t]{9}{*}{ COPD } & \multicolumn{2}{|c|}{ Mild: FEV1/FVC $<70 \%$ and FEV1 $\geq 80 \%$} \\
\hline & $\begin{array}{l}\text { Short Acting } \\
\text { Bronchodilators }\end{array}$ & $\begin{array}{l}\text { Albuterol, Levalbuterol, Ipatropium, and } \\
\text { Albuterol + Ipatropium }\end{array}$ \\
\hline & \multicolumn{2}{|c|}{ Moderate: FEV1/FVC $<70 \%$ and $50 \% \leq$ FEV1 $<80 \%$} \\
\hline & $\begin{array}{l}\text { Short Acting } \\
\text { Bronchodilators }\end{array}$ & As Above \\
\hline & $\begin{array}{l}\text { Long Acting } \\
\text { Bronchodilators }\end{array}$ & $\begin{array}{l}\text { Aclidinium, Arformoterol, Formoterol, Glycopyrrolate, } \\
\text { Idacaterol, Olodaterol, Salmeterol, Tiotropium, and } \\
\text { Umeclidinium }\end{array}$ \\
\hline & \multicolumn{2}{|c|}{ Severe: FEV1/FVC $<70 \%$ and $30 \% \leq$ FEV1 $<50 \%$} \\
\hline & $\begin{array}{l}\text { Short Acting } \\
\text { Bronchodilators }\end{array}$ & As Above \\
\hline & $\begin{array}{l}\text { Long Acting } \\
\text { Bronchodilators }\end{array}$ & As Above \\
\hline & $\begin{array}{l}\text { Inhaled } \\
\text { Corticosteroids }\end{array}$ & As Above \\
\hline
\end{tabular}


The most common medications used for long term control of asthma are inhaled corticosteroids (ICS), long acting beta 2 agonists (LABA) in combination with ICS, leukotriene modifiers (LTMs), anti-IgE therapy, and oral corticosteroids (discussed above). ICS are usually the first drug prescribed in addressing long-term control of asthma (Table 2) (99). ICS have been published to improve pulmonary function, decrease exacerbations and hospitalizations by $50 \%$ (compared to placebo or SABAs alone), and decrease asthma related deaths (when taking low doses regularly) $(94,100-103)$. ICS act by inhibiting airway inflammation through reduction of inflammatory gene transcription via reversing histone acetylation and recruiting histone deacetylase $(94,104)$. In addition, ICS also reduce the number of inflammatory cells (eosinophils, $\mathrm{T}$ cells, and dendritic cells) in the airways through down regulation of epithelial cell signaling (104). LTMs are less effective than the above treatments, due to their weak bronchodilator effect and thus are seldom used alone $(94,105)$. There are two types of LTMs, 5-lipoxygenase inhibitors which impair leukotriene synthesis and cysteinyl leukotreine-1 receptor antagonists which block the final destination of leukotriene (Table 2) (106). Anti-IgE therapy prevents the effect of IgE on immune cells by using an anti-IgE monoclonal antibody and subsequently reduces asthma exacerbations (Table 2) (107).

The non-pharmacological treatments for asthma are geared toward removing the person from the proximity of the extrinsic factors that trigger AHR (95). If segregation from the trigger is not possible, due to not knowing the trigger or inability to do so (economically, geographically, etc.), temperature sensitive laminar airflow devices can be administered to remove cold air from around the subject during sleeping (95). Lastly, 
psychologists may be of help to the patient in treating anxiety or depression to alleviate the effect of stress on asthma symptoms (95).

Bronchodilators and corticosteroids are also used in the management of COPD. However, it is important to again reiterate that COPD is an irreversible disease, so treatments are not geared for curing the disease but instead to help managing symptoms, slowing the loss of lung function, and reducing breathing difficulties (108). Smoking cessation is the most effective means for reducing disease progression, although, many smokers with COPD are undiagnosed and thus unaware that their habit is harming them drastically (28). Once a patient is diagnosed with COPD, treatment is based on the progression of the disease state as determined by spirometry and whether the symptoms are intermittent or persistent (Table 2) (108). Nonpharmacological treatments also aid in managing COPD, with smoking cessation being the most influential (108). Furthermore, oxygen therapy (if a patient is hypoxic) has been proven to increase survival of the patient and pulmonary rehabilitation (exercise training, educational, nutritional intervention, psychosocial support, and education) although underutilized, has been published to reduce hospitalizations and emergency room visits $(108,109)$.

It is important to emphasize that no treatment available today can cure asthma or COPD. This is likely due to the development of irreversible structural changes that perpetuate lung dysfunction. This project evaluates how perinatal exposures (nicotine and viral infection) may result in irreversible structural defects that persist into adulthood and enhance susceptibility to OADs depending on persistent exposures and genetic background. Addressing these perinatal effects will require further investigation with new 
models of research. Importantly, this concept suggests that the management of OADs is best delivered through prevention prior to disease development.

\section{Animal Models and Methods to Study OADs}

Clinical OAD cases are helpful to provide insight into the diseases and their treatment, but do not allow for full unravelling of the mechanisms behind the disease pathophysiology. Animal models have begun to bridge this gap in knowledge and are crucial to the development of therapeutic interventions including but not limited to OADs. Several animal models have been developed for the study of OADs. For asthma research, a variety of species have been implemented including fruit flies, rats, guinea pigs, cats, dogs, swine, cows, sheep, horses, and non-human primates, although the most widely used are mice (110). Asthma is not a murine disease and thus mice must become sensitized to increase allergen immunogenicity, which is followed by an allergen challenge (110). Murine models, after allergen challenge, mimic what is seen in humans showing increased IgE, epithelial hypertrophy, airway remodeling, $\mathrm{T}_{\mathrm{H}} 2$ immune response upregulation, and most importantly, ARH (110).

Animals models pertaining to COPD in the past have included hamsters, rats, guinea pigs, sheep, non-human primates and mice (111). Mice are the most commonly implemented animal model for emphysema (112). This model is achieved through intratracheal or aerosol inhalation of elastolytic enzymes such as porcine pancreatic elastase, human neutrophilic elastase, and papain. This model leads to alveolar septa destruction and results in lung functional abnormalities (increased airway resistance, decreased tissue elastance, decreased tissue damping, increased IC, increased FVC, and 
decreased PEF) as seen in humans $(113,114)$. Inducing chronic bronchiolitis in animals is most commonly conducted through intranasal or aerosolized inhalation of lipopolysaccharide (LPS) $(111,113,114)$. LPS leads to TLR signaling in the lungs, which leads to inflammation, immune cell infiltration (neutrophils, macrophages, and $\mathrm{CD}^{+} \mathrm{T}$ cells), and increases MMP-9/12 (113). In these models, LPS causes enlarged air spaces, thickening of alveolar walls, and lung functional abnormalities (increased airway resistance and decreased PEF) consistent to what is seen in humans $(113,114)$.

In addition to the above animal models, cigarette smoke exposure models are another option for researchers to use to investigate the pathophysiology of COPD. For this, rats, guinea pigs, and mice have been used although mice are the most extensively used animals $(111,113)$. Although this model would appear to be the model that correlates best to humans, there are several caveats such as standardized methods or protocols for exposure that have yet to be established (111). In addition, these models require a lot of investigator attention due to having to deliver smoke 5-7 days a week for a 6 month period (115). Furthermore, there is a plethora of variables between studies for this approach which include the type of cigarettes being used (research grade versus commercial or with versus without a filter), the delivery systems used (whole body versus nose-only), and the dose of smoke delivered $(111,113)$. Importantly, cigarette smoke inhalation models do not allow for a detailed examination of effects of individual tobacco components (e.g., nicotine). Despite all of this, cigarette smoke exposure models are correlative to humans cases of COPD, in regard to pulmonary infiltration of macrophages and neutrophils, fibrosis, and emphysema (111). 
The above models allow for evaluation of lung structure, while evaluating lung function is more difficult. Unlike most human subjects, an animal cannot be given directions to follow for PFT. Despite this, there have been methods/machines developed to assess pulmonary function such as with the SCIREQ flexiVent system, which is regarded as the gold standard for assessing lung functional measurements. Parameter outcomes in animals are acquired during tidal breathing or forced oscillation. The flexiVent system records measurements both at baseline and allows for administration of a drug or bronchoconstrictor such as methacholine. Typically, there are 6 parameters obtained, from two different forced oscillation techniques in the standard flexiVent equipment (Table 3). If desired a plethysmograph can be purchased to upgrade the flexiVent system in which the investigator can generate the FEV1, Forced Expiratory Flow (FEF), FVC, and the PEFR (of which are not identical to when they are recorded in humans) (116). Furthermore, software upgrades may also be purchased to obtain lung volumes such as the TLC, IC, and the RV. If an investigator wishes to measure the same animal's lung function more than one time in the subject's life the plethysmograph upgrade must be used. The reasoning for this is that the standard flexiVent equipment procedure requires euthanasia after testing, due to cutting the trachea for insertion of a blunt needle, to conduct the two forced oscillation techniques.

It is important to note that spirograms and flow-time graphs cannot be generated, although insights can be gained from the parameters obtained (Table 3). This is due to not being able to have maximum exhalation and inhalation being performed. Most frequently reported in the literature is increasing methacholine concentration. These figures are used to compare a treatment group to a control group, since a plethora of factors can influence 
lung function (genetic background, vendor source, and housing material), historical controls are not readily available in accessible databases $(117,118)$. Of most importance, is that the forced oscillation technique (single frequency versus broadband) allows investigators to determine the regions of the respiratory system being affected (large vs small airways and airways vs parenchyma) (Table 3). 


\section{Table 3. SCIREQ flexiVent Parameters}

\begin{tabular}{|ccl|}
\hline \multicolumn{1}{|c|}{ Parameter } & Abbreviation & \multicolumn{1}{c|}{ Definition } \\
\hline $\begin{array}{l}\text { Single Frequency Forced } \\
\text { Oscillation Technique }\end{array}$ & $\mathrm{R}$ & $\begin{array}{l}\text { Applies a sinusoidal waveform to the subject's airway opening } \\
\text { and resulting pressure, flow, and volume are fit to a single } \\
\text { compartment model using linear regression (116) }\end{array}$ \\
\hline Resistance & $\mathrm{C}$ & $\begin{array}{l}\text { Assess level of constriction in the respiratory system (114, 116, } \\
119)\end{array}$ \\
\hline Compliance & $\mathrm{E}$ & $\begin{array}{l}\text { Ease with which the respiratory system can be expanded (114, } \\
116)\end{array}$ \\
\hline Elastance & $\begin{array}{l}\text { The inverse of compliance, captures the elastic stiffness of the } \\
\text { respiratory system (116) }\end{array}$ \\
$\begin{array}{l}\text { Broadband Forced Oscillation } \\
\text { Technique }\end{array}$ & $\begin{array}{l}\text { Applies a broadband oscillation maneuver, in a stepwise } \\
\text { manner, to the subject's airway opening both above and below } \\
\text { the subjects breathing frequency. The resulting input } \\
\text { impedance is fit to a constant phase model to obtain differences } \\
\text { between airway and tissue mechanics (116) }\end{array}$ \\
$\begin{array}{l}\text { Newtonian } \\
\text { Resistance }\end{array}$ & $\mathrm{Rn}$ & Resistance of the central or conducting airways (114, 116, 120) \\
$\begin{array}{c}\text { Tissue } \\
\text { Damping }\end{array}$ & $\mathrm{G}$ & Resistance of the parenchymal airways (114, 116, 119-121) \\
\hline $\begin{array}{c}\text { Tissue } \\
\text { Elastance }\end{array}$ & $\mathrm{H}$ & Elastance of the parenchymal airways (116, 119, 120) \\
\hline
\end{tabular}


Once abnormal lung function is observed in an animal, several other methods may be employed to determine what is driving these changes. Raw histology of lungs can be used to obtain gross observation to determine how differences in spirometry are occurring. To elaborate, Masson's Trichrome staining can be used to assess the amount of collagen, while Hematoxylin and Eosin (H\&E) staining can be used to determine the architecture of the airways and to determine the alveolar volume. Immunohistochemistry (IHC) can be used to examine for the presence of an antigen of interest or produced molecules. The caveat of using histology is that it is subject to biases of the researcher, for which attention should be given to paraffin embedding orientation of tissues, cutting tissue sections, and determining what sections to stain. To alleviate biases, morphometric analysis may be employed to ensure an overall representation of the lung architecture is being assessed through randomized selection of lung regions. Using histology, one may use Masson's Trichrome, H\&E, and/or IHC staining and quantitate either manually or with computer generated software programs (such as Image J). Transcripts profiles are also of importance, which are achieved through using RT-PCR or microarray analysis, although the latter is more thorough and expensive. In addition, western blotting can also be implemented to determine differences in protein concentration although this method is not as sensitive as the above. Taken together these methods can allow one to begin to unravel the forces driving disease states in animal models to further societies understanding of OADs.

\section{Project Goals and Hypothesis}

The above evidence generated from animal models, human studies, and epidemiological data led us to address the question whether perinatal nicotine exposure 
and/or early life influenza infection promote chronic lung disease in the adult reminiscent of OADs (Fig. 4). We hypothesized that perinatal nicotine and early life influenza infection alone would lead to lung structural and functional abnormalities reminiscent to a disease state present in humans, and that combinations of nicotine and influenza would potentiate the effects seen with either factor alone. To test our hypothesis, we built upon our previous published murine model for perinatal nicotine exposure and developed a new murine model for early life influenza infection in mice whose lung developmental state resemble individuals who had the highest prevalence for the 2009 pandemic influenza infection. This work showed lung functional abnormalities driven by phenotypic alterations, established and characterized a new early life model for infection with pandemic influenza infection which results in abnormal lung function, and used both exposures together to test our hypothesis. 


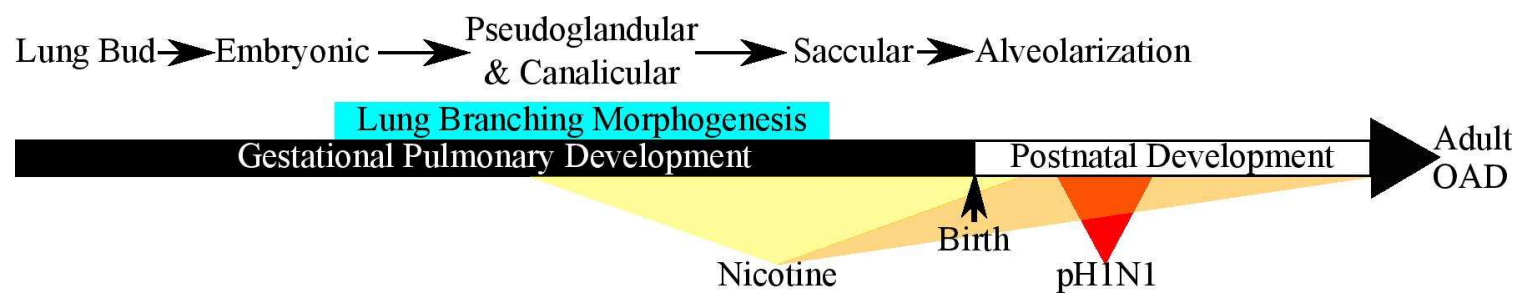

Figure 4. Potential Effects of Early Life Exposures on Lung Development

After the lung bud is formed from the foregut, the primordial lung growths and branches during the process of lung branching morphogenesis, which is responsible for the formation of the large airways during the pseudoglandular stage of lung development. Cells within these airways engage in vasculogenesis, while endothelial cells invade the lung through angiogenesis resulting in the development of the early circulatory system during the canalicular stage of lung development. Afterwards, the distal lung branches develop alveolar sacs which ultimately transform into fully functional gas exchanging units during the alveolar stage; the latter spans before and after birth. Each of these overlapping processes are dependent on the orchestrated and fine-tuned expression and repression of extracellular matrices and growth factors and morphogens, among other molecules. Exposures during distinct stages might impact distinct biological pathways, thereby impacting the development of specific pulmonary structures, which if not corrected, persists until adulthood. For example, exposure during the pseudoglandular stage (e.g., nicotine) may impact lung branching morphogenesis, while exposures early during the alveolar stage (e.g., viral infection) may impact alveolar formation 
CHAPTER II: EARLY LIFE NICOTINE EXPOSURE LEADS TO STRUCTURAL AND FUNCTIONAL ABNORMALITIES IN THE ADULT MURINE LUNG 


\section{$\underline{\text { Background }}$}

COPD affects millions worldwide resulting in over $\$ 50$ billion dollars in healthcare costs in the U.S. alone in 2010, which is predicted to increase $(10,122)$. In the same period, COPD caused more days of lost productivity than any other chronic condition, costing more than $\$ 4$ billion (122). It is estimated that $40 \%$ of the annual cost due to COPD could be avoided by preventing complications and hospitalizations through the development of effective treatments (123). The development and/or exacerbation of COPD and other obstructive airway diseases is mostly attributed to tobacco smoke exposure $(124,125)$.

The United States Surgeon General (USG) report in 1986 (C. Everett Coop) focused on "The Health Consequences of Involuntary Smoking" and concluded that there was sufficient evidence that tobacco smoke exposure was associated with adverse health effects in children and adults, increased the frequency of hospitalizations due to respiratory tract infections as well as bronchitis and pneumonia in children, and decreased lung function measurements in children compared to those whose mothers did not smoke tobacco (124). Epidemiological data generated since then have strongly supported the USG reports notions, showing that maternal smoking leads to an increased risk for asthma and viral respiratory tract infections, decreased lung function, and wheezing in infants $(42,47,64$, 124, 126). Despite the above, the Centers for Disease Control and Prevention and others estimate that $12-25 \%$ of women continue to smoke during pregnancy $(57,125,127-129)$.

It has been proposed that the development of chronic obstructive lung disorders is greatly influenced (and perhaps pre-determined) by adverse exposures/events in early life $(3,4,46,47,130-132)$. Stern and colleagues, for example, evaluated individuals during their first three decades of life and grouped individuals into quartiles based on their lung 
function. They found that individuals who were included in the group with the lowest lung function as children were also found in the group with the lowest lung function as adults (43). Furthermore, children that suffer from severe, persistent asthma are thirty-two times more likely to develop COPD as adults $(4,133)$. Cohen et al. suggested that in utero tobacco smoke exposure reduces age-related improvements in lung function among children with asthma, thus predisposing them to COPD as adults (130). These and other observations strongly suggest early life adverse events, such as tobacco smoke exposure, promote adaptations that lead to abnormal lung function later in life. However, despite the perceived importance in the development of pulmonary disease, the exact mechanisms responsible for the effects of tobacco smoke and the components of tobacco responsible for these effects remain incompletely elucidated.

Nicotine is a constituent of tobacco that is responsible for its addictive qualities (42, 57, 64). This plant alkaloid readily transverses the placenta, and fetal levels of nicotine or its metabolite cotinine are significantly greater than in the mother $(57,61)$. Our laboratory has been interested in studying how gestational, perinatal, or postnatal nicotine exposures impact the lung, and previously reported that murine maternal perinatal nicotine exposure affects lung branching morphogenesis, which led to airway dysfunction in the young animal ( 8 weeks of age) $(42,64)$. This loss of lung function was postulated to be driven by alterations in extracellular matrix expression and deposition, among other changes, resulting in dysynaptic lung growth (42). Our findings were consistent with studies conducted in non-human primates and in rats where nicotine exposure led to increased collagen deposition and lung functional abnormalities in the young offspring $(63,134$, 135). However, the question remained whether these early changes caused by nicotine 
persist to promote structural and functional abnormalities in the adult lung. Thus, we set out to determine if early life nicotine exposure would promote structural and functional abnormalities in the adult lung. We discovered that chronic nicotine exposure starting during the perinatal period caused significant changes in lung function and structure.

\section{Materials and Methods}

\section{Murine Housing and Breeding}

Mice were housed in a specific pathogen-free barrier facility accredited by the Association for Assessment and Accreditation of Laboratory Animal Care, and procedures were approved by the University of Louisville's Institutional Animal Care and Use Committee. Timed breeding was implemented by pairing C57BL/6 (Jackson Laboratories Bar Harbor, ME) mice and un-pairing within 48 hours. Mice were examined for pregnancy at 14-days post pairing, at which time nicotine was administered. Mice were housed with their mother until 28-days of age on 5021 chow. Upon weaning mice at 28-days of age, the food was changed to 5010 formulation. Mice were weighed daily within a two-hour time frame until 51 days of age, at which time blood was drawn and mice were given an intraperitoneal injection of sterile saline at $0.5 \mathrm{~mL}$ (GrowCells \#MSDW-2000 Irvine, CA). Serum was used to test for seroconversion against H1N1 (My BioSource \#MBS9350017 San Diego, CA), since mice infected with influenza were housed in the same facility.

\section{Nicotine Exposure}

Female breeders were administered nicotine (Millipore Sigma \#N3876 St. Louis, MO) via the drinking water at $100 \mu \mathrm{g} / \mathrm{mL}$ (protected from light) before timed mating. 
Upon breeding, no nicotine was added to the water until gestational day 14, when females were checked for pregnancies and water was again supplemented with nicotine as before. Nicotine remained in the water until the offspring reached 7-days of age or 210-days of age $(42,136)$. Previously, we showed that exposure to nicotine during the pseudoglandular stage of lung development and up to 7-days post birth was sufficient to promote structural and airway functional changes in this experimental model, which explains the timing used for the first group (transient nicotine exposure). This was then compared to prolonged nicotine exposure group that started during gestation at the same time, but continued until 7 months of age (chronic nicotine exposure) (42).

\section{Murine Lung Function Testing}

Untreated mice $(n=19$; male $=10$, female $=9)$, mice in the transient nicotine exposure group $(\mathrm{n}=18$; male $=10$, female 8$)$, and mice in the chronic nicotine exposure group $(\mathrm{n}=20 ;$ male $=10$, female $=10)$ were subjected to pulmonary function testing. Nicotine water was removed approximately $2-8$ hours before testing. Pulmonary function at baseline and after aerosolized methacholine challenge $(0-50 \mathrm{mg} / \mathrm{mL}$, inhaled) was measured by forced oscillation using the flexiVent system (SCIREQ Montreal, Quebec, Canada) as previously described $(137,138)$.

\section{Tissue Processing and Analysis}

The chest cavity was then opened and the inferior vena cava was cut. The lung vasculature was flushed by puncturing the right ventricle with a $0.26 \mathrm{G}$ needle and injecting $10 \mathrm{~mL}$ sterile saline solution. Lungs were inflated via the trachea with $10 \%$ neutral buffered formalin (VWR \#16004-128 Randor, PA) at a constant pressure of $25 \mathrm{~cm} \mathrm{H}_{2} \mathrm{O}$. Inflated 
lungs were removed and placed in $10 \%$ neutral buffered formalin for storage until separation of individual lobes then processed using a spin tissue processor 120 (Thermo Fisher \#813150 Waltham, MA) according to the manufacturer's instructions $(42,64)$.

Lobes were then paraffin embedded and were then cut at a thickness of $6 \mu \mathrm{m}$ (Leica Jung \#RM2055 Wetzlar, Germany) taking horizontal sections of all lobes. Slides were then stained using standard procedures for H\&E (Thermo Fisher \#6765007 Waltham, Massachusetts) (Thermo Fisher \#6766007) and Masson's trichrome (MTC) (American MasterTech \#KTMTR2PT Lodi, CA). After staining tissue sections were dehydrated using standard procedures and then mounted with Vectamount mounting medium (Vector Laboratories \#H5000 Burlingame, California). Quantification of collagen was conducted using ImageJ (Version 1.51k) software as previously described $(139,140)$. For our analysis, we applied a minimum threshold level of 37 and a maximum threshold level of 190 for our Masson's trichrome stained slides (collagen). To evaluate alveolar structure, three images (20x) were taken of the left lung parenchyma from each mouse. Images were obtained using a fixed frame for all images to ensure the areas being quantitated were of exact, equal size among all specimens. Alveolar septa were quantitated by manually counting the number of times the alveolar septa intersected with the lines on a uniform coherent $25 \times 25$ grid (both vertically and horizontally) in Inkscape as previously described $(141,142)$. Data from three images were used to calculate a single mean linear intercept value for each mouse as previously described $(141,142)$; these values were then used to determine averages and standard deviations for treatment groups for statistical analysis. 


\section{Statistics}

SAS statistical software was used to analyze lung function data. A correlation chart for pairwise comparison between all six outcome parameters (resistance, compliance, elastance, Newtonian resistance, tissue damping, and tissue elastance) was generated and showed strong correlation for all parameters to resistance except for Newtonian resistance (Fig. 5). A 3-factor ANOVA was then implemented with the factors representing experimental group, sex type, and dose of methacholine used. Descriptive statistics were calculated for the entire study population as well as within each factor. Rigorous analysis involved the main effects for 3-factor ANOVA along with 2-way interactions (group*sex, group*dose, sex*dose). Since interaction effects were not significant in most of the outcome measures, we considered only 3-factor ANOVA without interaction terms. Rather than subset analysis, we have used contrast to test the main effects (such as untreated versus acute nicotine; untreated vs chronic nicotine, and acute versus chronic nicotine), which increases power to detect significant effects $(143,144)$.

Using GraphPad Prism 7.03 the following statistical test were implemented. The average daily weights were calculated along with standard deviation then compared using two-tailed unpaired T-tests along with a Holm-Sidak test to correct for multiple comparisons. In addition, a linear regression analysis was also implemented testing whether slopes and intercepts were different between weights at 34 and 210-days of age (data not shown). Raw data from mean linear intercept quantification were analyzed using one-way ANOVA analysis with a Bonferroni post-test to correct for multiple comparisons or two-tailed unpaired t-tests compared to untreated controls. The percent of total lung area stained (entire lung) for each group was averaged along with standard deviations from 
Image $\mathrm{J}$ analysis, and a one-way ANOVA using a Bonferroni test to correct for multiple comparisons (compared to untreated mice) or unpaired two-tailed t-tests analysis (compared to untreated) was performed. 


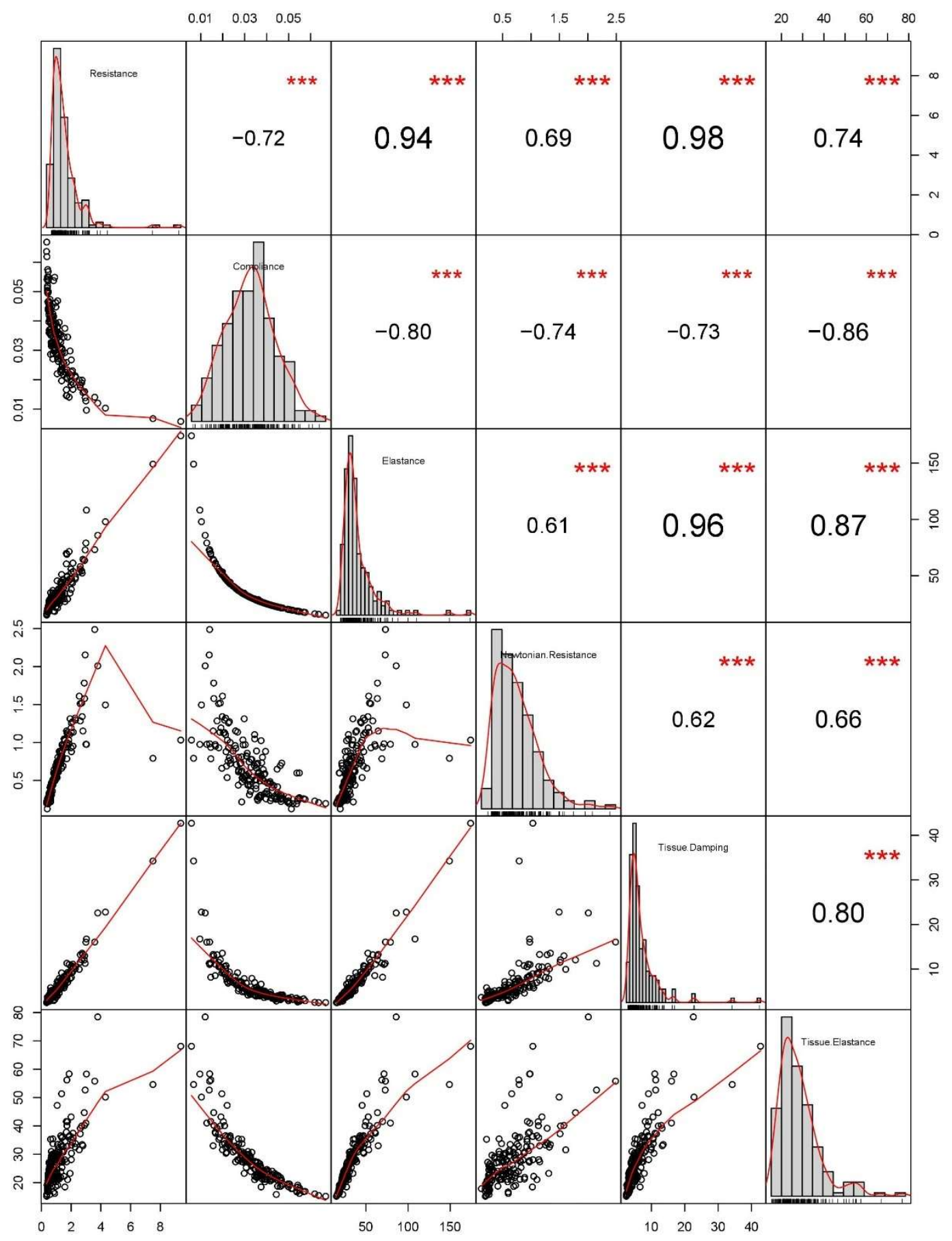

Figure 5. Correlation of Pulmonary Function Test Outcome Parameters for

\section{Perinatal Nicotine Statistical Analysis (All Groups)}

All data used for analysis in SAS 3 Factor ANOVA. Graphical representation of correlation represented in bottom left of figure. Fit of data to linear line of regression represented in top right of figure. 


\section{$\underline{\text { Results }}$}

\section{Nicotine exposure alters lung function}

PFTs were conducted at 210-days of age in mice exposed to nicotine transiently (pre-birth to 7-days of age) or chronically (pre-birth to adulthood) along with untreated animals (Fig. 6). Resistance, compliance, elastance, Newtonian resistance, tissue damping, and tissue elastance were examined at baseline and after stimulation with increasing concentrations of aerosolized methacholine from $0-50 \mathrm{mg} / \mathrm{mL}$. For statistical analysis, we only evaluated responses at baseline and after 25 and $50 \mathrm{mg} / \mathrm{mL}$ of methacholine. First, we determined that a strong correlation was occurring between the outcome parameters tested with exception of Newtonian resistance (Fig. 5). Further evaluation revealed that chronic nicotine exposure compared to untreated animals, caused a statistically significant increase in compliance $(\mathrm{p}<0.001)$, a decrease in Newtonian resistance $(\mathrm{p}=0.003)$, and a decrease in tissue elastance $(\mathrm{p}=0.041)$ (Fig. 7) (summarized in Table 4). Furthermore, differences were found between all methacholine exposures. Of the differences discovered between the untreated and chronic nicotine exposure groups, increased compliance and decreased tissue elastance were dependent on sex with changes in males accounting for much of the differences found $(\mathrm{p}<0.001)$ (Fig. 7) (Table 4). In contrast to chronic nicotine exposure, transient nicotine exposed mice group showed no differences compared to the untreated (Table 4). However, one male mouse in the transient nicotine exposed group showed extremely abnormal lung function at $50 \mathrm{mg} / \mathrm{mL}$ methacholine dose with compliance and elastance measurement being, 0.006 and 174.2, tissue elastance being 68.11, while Newtonian resistance remained relatively normal (1.031). 


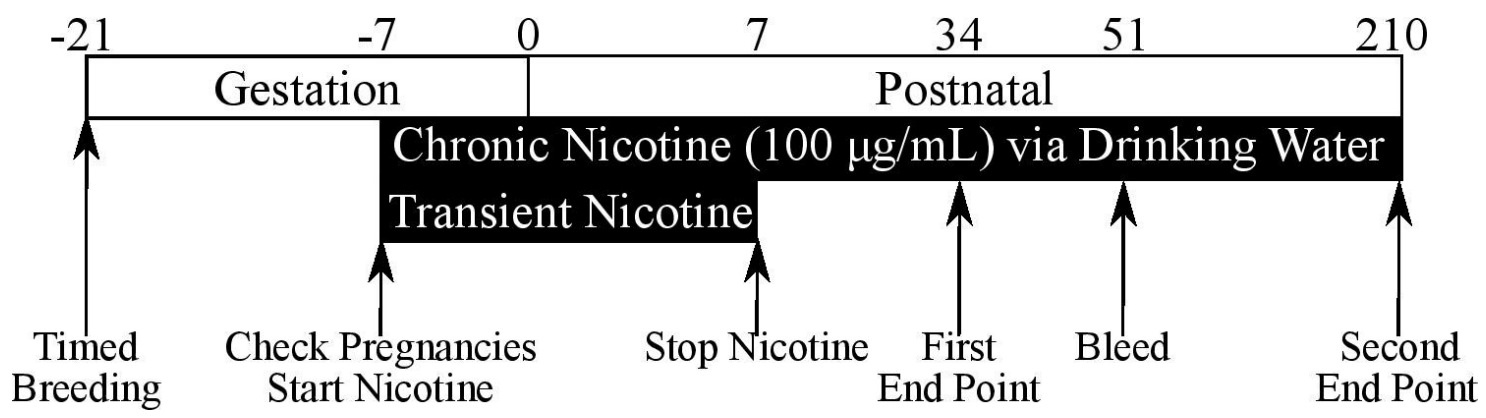

\section{Figure 6. Perinatal Nicotine Exposure Experimental Design}

Timed pregnancies were produced by pairing mice for 48 hours and then assessing for conception after 14 days, at which point nicotine was administered via the drinking water at $(100 \mu \mathrm{g} / \mathrm{mL})$ for pregnant mothers. Nicotine exposure lasted until postnatal day 7 or until the completion of the experiment (postnatal day 210), when pulmonary function tests were conducted. Endpoints were implemented at 34 and 210-days of age. 

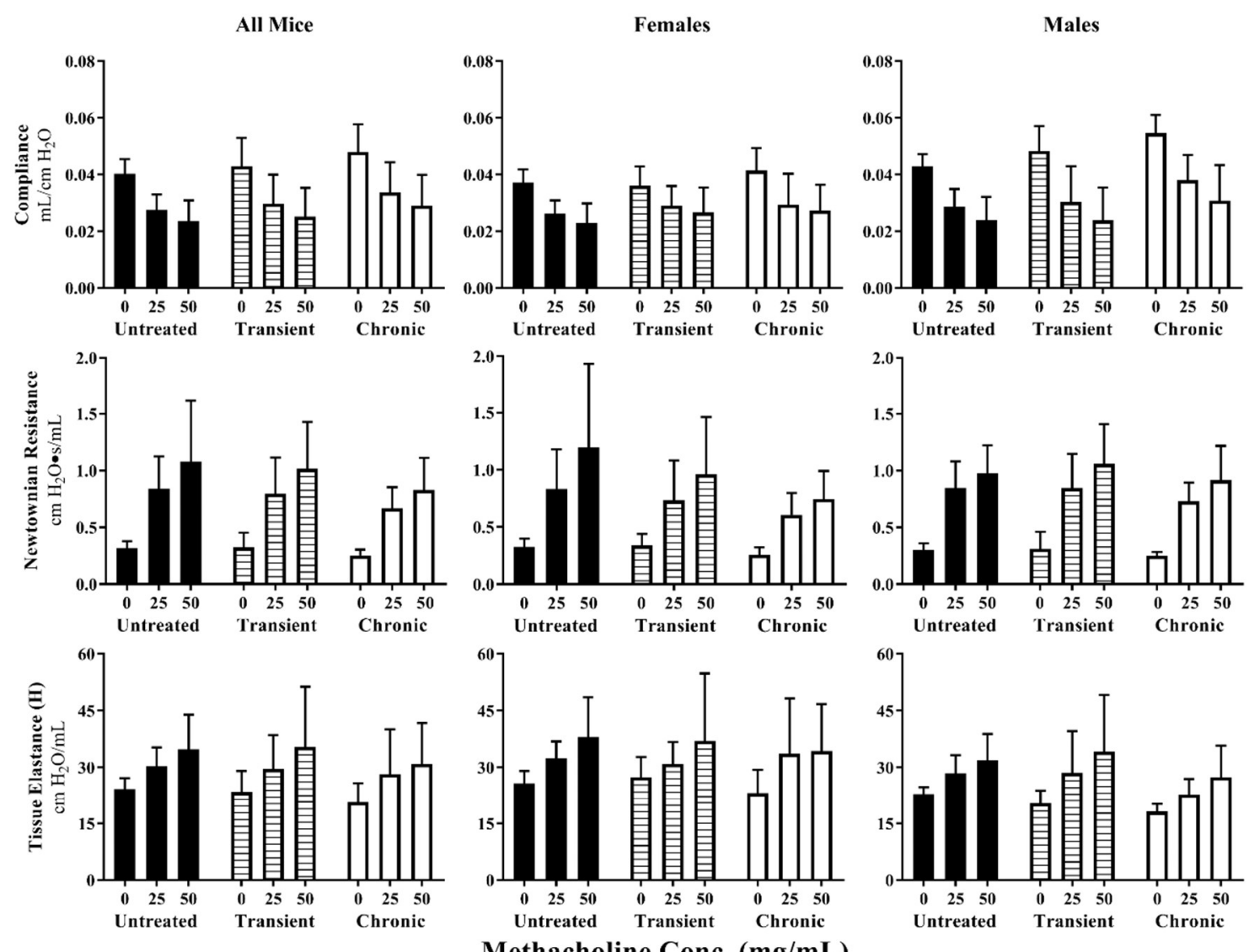

Methacholine Conc. $(\mathrm{mg} / \mathrm{mL})$

\begin{tabular}{|l|c|c|c|c|c|c|}
\hline Comparison & Resistance & Compliance & Elastance & Newtonian Resistance & Tissue Damping & Tissue Elastance \\
\hline Untreated vs. Transient & 0.239 & 0.214 & 0.495 & 0.543 & 0.205 & 0.947 \\
\hline Untreated vs. Chronic & 0.122 & $<.001 \uparrow$ & 0.169 & $0.003 \downarrow$ & 0.140 & $0.041 \downarrow$ \\
\hline Transient vs. Chronic & $0.007 \downarrow$ & $0.005 \uparrow$ & $0.042 \downarrow$ & $0.021 \downarrow$ & $0.007 \downarrow$ & 0.051 \\
\hline $\mathbf{0}$ vs. 50 mg/mL & $<.001 \uparrow$ & $<.001 \downarrow$ & $<.001 \uparrow$ & $<.001 \uparrow$ & $<.001 \uparrow$ & $<.001 \uparrow$ \\
\hline $\mathbf{0}$ vs. 25 mg/mL & $<.001 \uparrow$ & $<.001 \downarrow$ & $<.001 \uparrow$ & $<.001 \uparrow$ & $<.001 \uparrow$ & $<.001 \uparrow$ \\
\hline $\mathbf{2 5}$ vs. 50 mg/mL & $0.024 \uparrow$ & $0.007 \downarrow$ & $0.020 \uparrow$ & $<.001 \uparrow$ & $0.025 \uparrow$ & $0.010 \uparrow$ \\
\hline Female vs. Male & 0.214 & $<.001 \uparrow$ & 0.567 & 0.548 & 0.657 & $<.001 \downarrow$ \\
\hline
\end{tabular}

Figure 7. Pulmonary Function Test Results for Nicotine Exposure at 210-Days of Age

Lung function was assessed C57BL/6 at 210-days of age by forced oscillation technique using a SCIREQ flexiVent system. Lung function was measured at baseline and after challenge with increasing concentrations of aerosolized methacholine. Averages of results (only including values where the COD was $\geq 95 \%$ ) were taken for each individual mouse. SAS statistical software was used to conduct 3-factor ANOVA without interaction terms. Results were declared significant at 5\% significance level $(\mathrm{p}<0.05)$. Error bars represent standard deviation from the mean. P-values are represented in the table with significant results having an arrow dictating the direction of the second group in each row compared to the first group. 
Table 4. Summary of Nicotine Pulmonary Function Testing

\begin{tabular}{|c|c|c|c|c|c|c|}
\hline \multicolumn{2}{c}{ Resistance } & Compliance & Elastance & $\begin{array}{c}\text { Newtonian } \\
\text { Resistance }\end{array}$ & $\begin{array}{c}\text { Tissue } \\
\text { Damping }\end{array}$ & $\begin{array}{c}\text { Tissue } \\
\text { Elastance }\end{array}$ \\
\hline $\begin{array}{c}\text { Nicotine } \\
\text { Transient }\end{array}$ & $\begin{array}{c}\text { No } \\
\text { Change }\end{array}$ & No Change & $\begin{array}{c}\text { No } \\
\text { Change }\end{array}$ & No Change & $\begin{array}{c}\text { No } \\
\text { Change }\end{array}$ & $\begin{array}{c}\text { No } \\
\text { Change }\end{array}$ \\
\hline $\begin{array}{c}\text { Nicotine } \\
\text { Chronic }\end{array}$ & $\begin{array}{c}\text { No } \\
\text { Change }\end{array}$ & $\uparrow$ & $\begin{array}{c}\text { No } \\
\text { Change }\end{array}$ & $\downarrow$ & $\begin{array}{c}\text { No } \\
\text { Change }\end{array}$ & $\downarrow$ \\
\hline
\end{tabular}

Arrows indicate direction of parameter compared to untreated-mock infected control Blue indicates alteration in function driven by males

Black indicates alteration in function not driven by either sex 


\section{Nicotine exposure alters lung structure}

The lungs of mice assessed for lung function were examined by H\&E and Masson's Trichrome staining. As depicted in Figure 8A, both transient and chronic nicotine exposure resulted in larger airspaces when compared to untreated animals. However, quantification of alveolar mean intercept revealed that only chronic nicotine exposure resulted in a significantly decreased number of intersections consistent with decreased alveolar surface area and increased alveolar size when compared to untreated controls (Fig. 8C). Interestingly, when examining groups by sex, we discovered that the observed effects of both transient and chronic nicotine exposure predominantly occurred in males, while females manifested changes only in the chronic nicotine exposure groups (Fig. 8C). Altogether, increases in alveolar volume occurred in a uniform manner throughout the lung in each group at 210-days of age. Length between the alveolar septum was approximately $50 \%$ greater for chronic nicotine versus untreated controls.

Masson's Trichrome staining showed increased collagen staining mostly located around large airway structures. As presented in Figure 8B, chronic nicotine exposure resulted in increased collagen deposition (blue color) when compared to untreated and transient nicotine treatment groups. Collagen deposition occurred primarily around large central conducting airways, with approximately of $30-50 \%$ of airways affected. Quantification of collagen staining revealed more collagen deposition in the chronic nicotine treatment group when compared to untreated controls, with both females and males experiencing the effect (Fig. 8D). In contrast, no differences were detected in the transient nicotine treatment group when compared to untreated controls. 


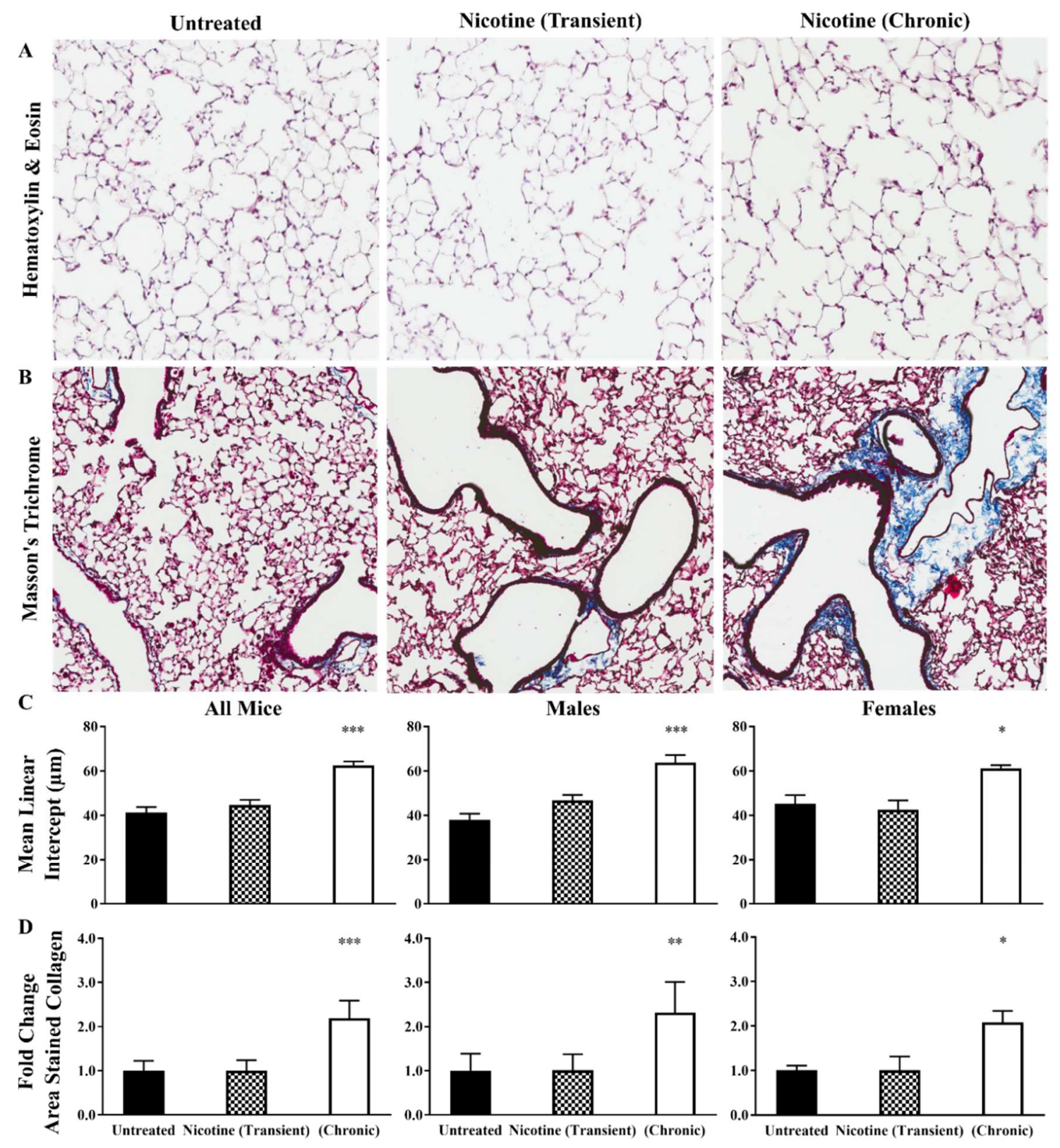

\section{Figure 8. Lung Histology of Nicotine Exposed Mice at 210-Days of Age}

Lung histology in C57BL/6 mice, including H\&E staining at 20x magnification (A) and Mason's Trichrome staining at 10x magnification (B). Alveolar septum mean linear intercept quantification and the percent of total lung area stained for collagen (C\&D). In GraphPad Prism 7.03 two-tailed t-tests and one-way ANOVA analyses were conducted with all groups using a Bonferroni test to correct for multiple comparisons. Multiplicity adjusted p-values were reported for each comparison. Error bars correspond the standard 
deviation $(\mathbf{C})$ or error $(\mathbf{D})$ the mean. Differences are represented vs. mock infected controls, $\mathrm{p}<0.05$ : *, p $<$ $0.01:^{* *}, \mathrm{p}<0.001:^{* * *}$. 


\section{Nicotine exposure affects lung structure in the young lung}

Because major functional and structural pulmonary changes were not observed in the transient nicotine exposure group, we focused our attention on the chronic nicotine exposure group. Specifically, we set out to investigate if nicotine led to pulmonary changes early in life that persisted into adulthood. For this, we examined the histology of untreated and nicotine-treated lungs (chronic) at 34-days of age (Fig. 6, endpoint \#1). As presented in Figure 9A, the lungs of nicotine-treated animals showed enlarged airspaces at 34-days of age, which was confirmed by evaluating the alveolar mean linear intercept (Fig. 9C). Notably, this effect was again mainly driven by changes in males rather than females (Fig. 9C). Interestingly, the alveolar mean linear intercept measured at 34-days of age was greater for animals with chronic nicotine exposure when compared to the findings detected at 210-days of age suggesting continuing alterations in lung structure until adulthood (Fig. 10). At 34-days of age the increase in alveolar volume occurred in a uniform manner within groups. Although for chronic nicotine, the alterations were less uniform for a limited number of mice. Altogether, there was an approximate $10-20 \%$ increase in length between the alveolar septum of chronic nicotine versus untreated groups. However, when collagen was evaluated, no differences were detected when comparing untreated mice and mice chronically exposed to nicotine at 34-days of age (Fig. 9D). 


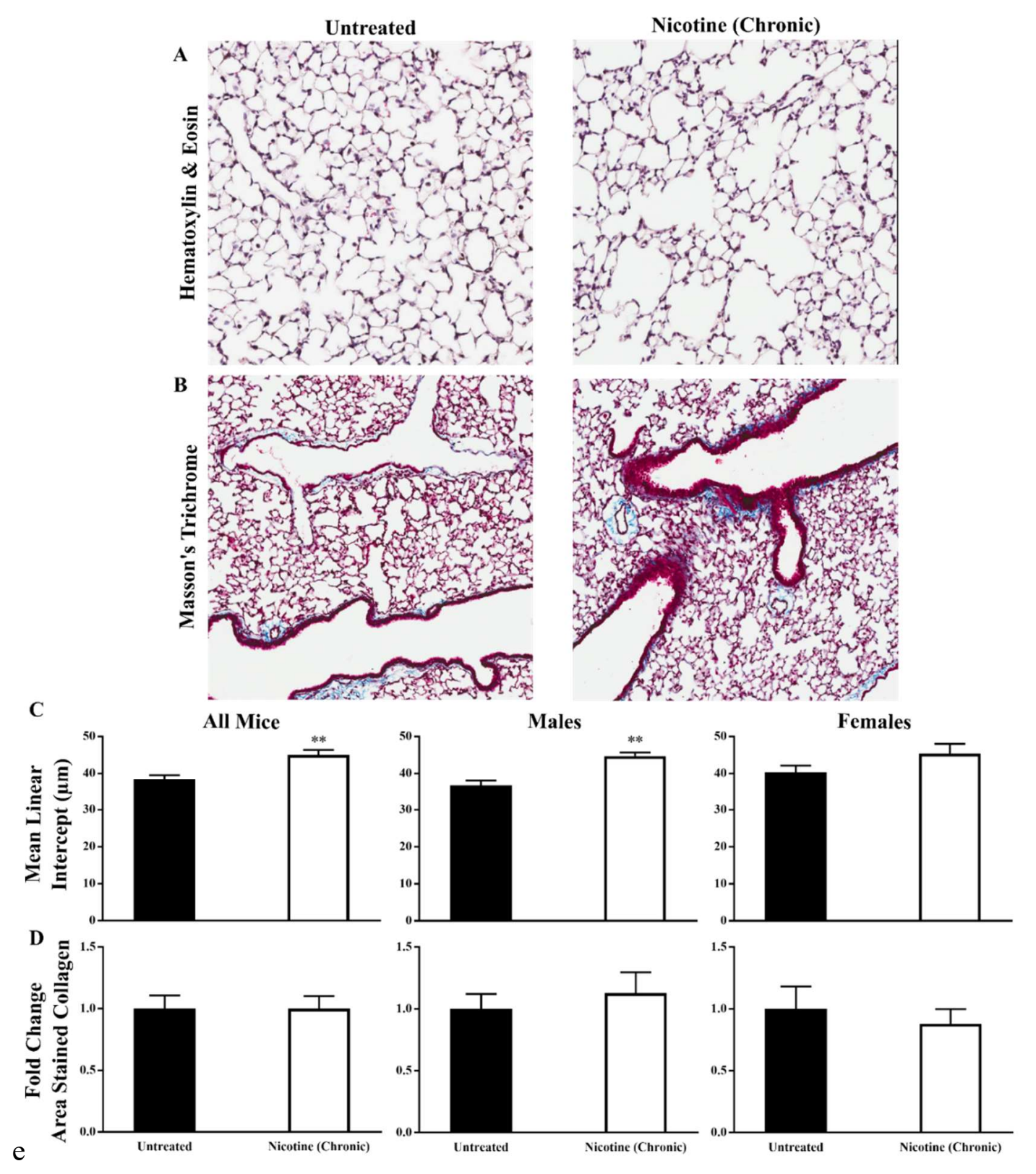

Figure 9. Lung Histology of Chronic Nicotine Exposed Mice at 34-Days of Age

Lung histology in C57BL/6 mice, at 34-days of age, including H\&E staining at 20x magnification (A) and Masson's Trichrome staining at 10x magnification (B). Alveolar septum mean linear intercept quantification (C) and the percent of total lung area stained for collagen (D). In GraphPad Prism 7.03 two-tailed t-tests were conducted. Multiplicity adjusted p-values were reported for each comparison. Error bars correspond the standard deviation $(\mathbf{C})$ or error (D) of the mean. Differences are represented vs. mock infected controls, $\mathrm{p}<0.05: *, \mathrm{p}<0.01:^{* *}, \mathrm{p}<0.001:^{* * *}$. 
A

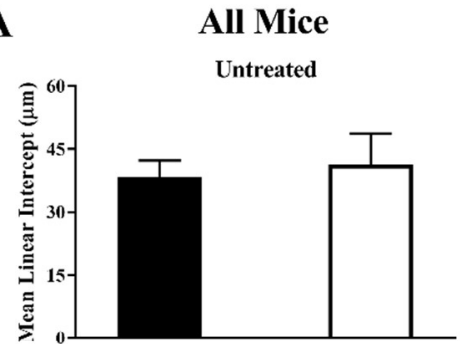

B

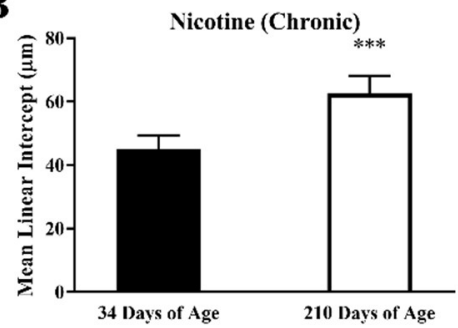

Males
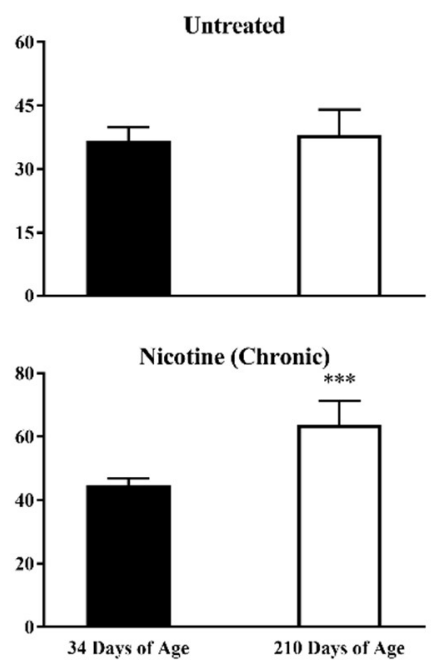

Females

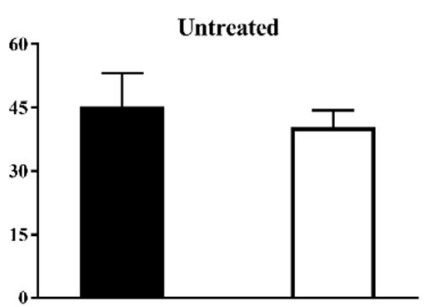

Nicotine (Chronic)

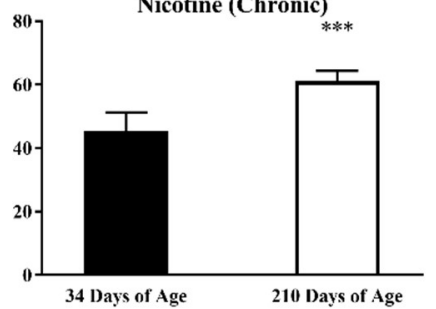

Figure 10. Alveolar Septum Quantification Over Time in Chronic Nicotine Exposed

\section{Mice}

Alveolar septum mean linear intercept quantification within groups over time for untreated (A) and chronic nicotine (B) exposed groups. Two-tailed T-tests analyses were conducted with all groups compared to untreated controls. Multiplicity adjusted p-values were reported for each comparison. Error bars correspond the standard deviation of the mean. Differences are represented at 34 vs 210 days of age, $p<0.001$ : ***. 


\section{Effects of nicotine on mouse weight}

We were concerned that mice exposed to nicotine might show decreased food intake and, ultimately, lower weight. Since this could impact lung growth and maturation, we followed body weights in untreated and nicotine-treated mice later tested for lung function $(145,146)$. As shown in Figure 11, mice gained weight independent of their treatment group and increases in weight did not appear affected by transient or chronic nicotine exposure. When evaluating treatment effects on different sexes, weights in male mice showed no differences among treatment groups, whereas female mice exposed to nicotine had a slightly larger mean body weight, which was statistically higher than controls at 34 and at 210-days of age (chronic only) (Fig. 11B\&C). However, although differences were discovered in female mice weights, there was no difference in weight gain between controls and nicotine-exposed groups when or when not taking sex into consideration (data not shown) (Fig. 11). 
A All Mice

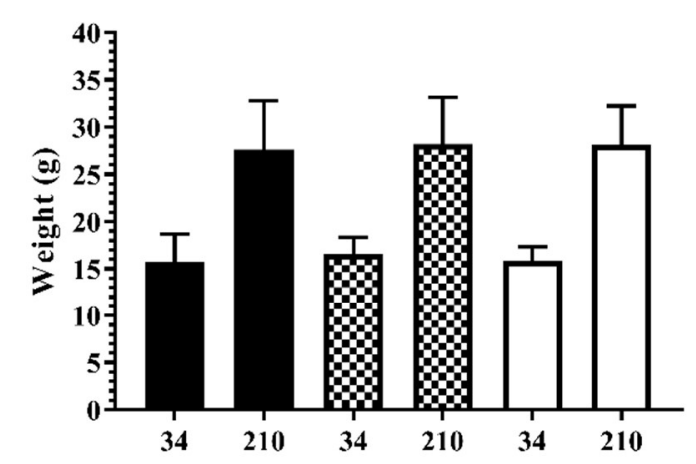

B

Male Mice

C

Mock

B Nicotine Acute

$\square$ Nicotine Chronic

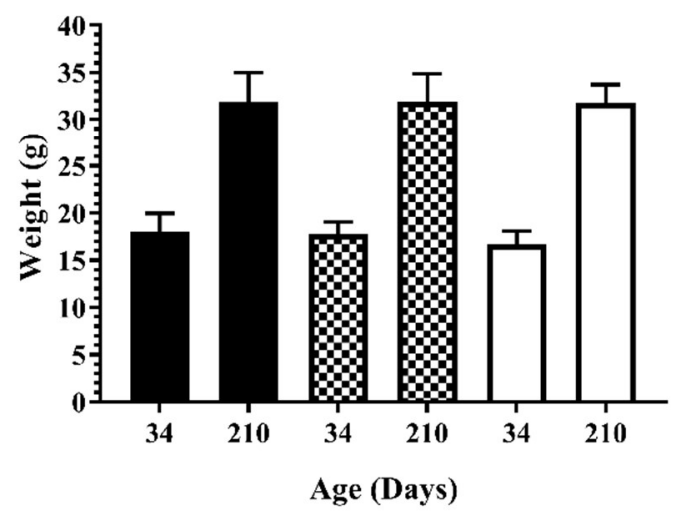

Female Mice

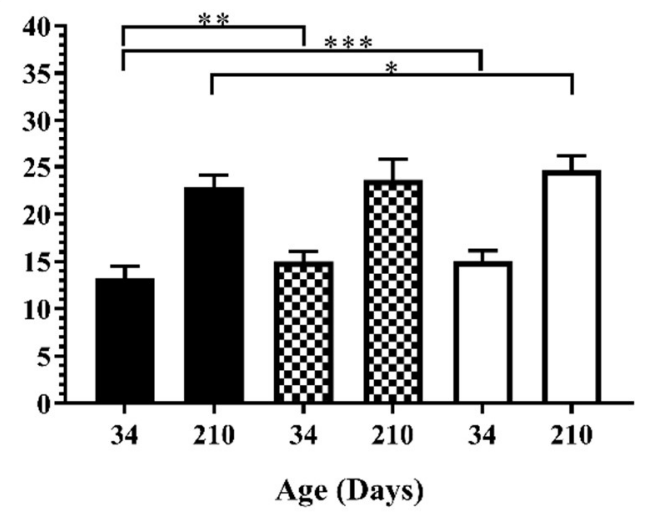

Figure 11. Weights of Nicotine Exposed Mice Assessed for Lung Functional

\section{Abnormalities}

Average weights of mice groups for the indicated exposures: untreated is shown in black, transient nicotine is shown in grey, and chronic nicotine is shown as unfilled or white. Individual weights were imported into GraphPad Prism 7.03, in which the groups were compared using unpaired t-test analysis with a Holm-Sidak multiple comparison test. A Linear Regression analysis was also conducted between groups in GraphPad, with no differences found. Multiplicity adjusted p-values were reported for each comparison. Differences are represented vs. mock infected controls, $\mathrm{p}<0.05:{ }^{*}, \mathrm{p}<0.01$ : $^{* *}, \mathrm{p}<0.001$ : *** 


\section{$\underline{\text { Discussion }}$}

Since the first report by Dr. Luther L. Terry in 1964, USG reports have played a vital role in pivoting the public view on smoking tobacco. In 1972, the USG began dissecting the health consequences due to both voluntary and involuntary tobacco smoke exposure on both respiratory and cardiac diseases. Shortly after in 1975, nicotine became implicated as a contributing factor for diseases in non-smokers exposed to second-hand tobacco smoke. However, it was not until the USG report of 1986 that the adverse effects of parental smoking on their children's respiratory health (most notably, increased frequency of bronchitis and pneumonia and subtle differences in lung function, all found early in life) were explained. Despite its perceived significance, and although it has been over 30 years since the USG 1986 report, the mechanisms driving the long-term consequences of tobacco smoke on lung function and structure remain unelucidated.

Several human epidemiological studies have looked at the effect and impact of environmental tobacco smoke and nicotine on lung development and the progression of OADs. In the United States, it has been estimated over a decade (2000-2010) that 400,000 children are born to mothers who have smoked during pregnancy (125). Smoking during pregnancy or more specifically fetal nicotine exposure (in animal models) has been shown to result in the offspring to have decreased lung function in childhood, which subsequently causes lifelong lung functional abnormalities $(42,43,48-52,62,63,134,135,147)$. A study of a European birth cohort found that maternal smoking during the first trimester alone caused a 2-fold increase in childhood asthma, and the risk for asthma development remained elevated into adulthood $(148,149)$. Furthermore, children whose mothers smoked during pregnancy were published to have decreased respiratory flows, decreased 
lung compliance, and altered tidal breathing $(50,150,151)$. Despite this knowledge in is estimated that $50 \%$ of smokers who become pregnant continue to smoke during pregnancy, in which in the United States the highest prevalence, from 2000-2010, was found to be in 20-24 year old mothers $(48,125)$. Furthermore, postnatal exposures to tobacco smoke also continues to be widespread with an estimated $40 \%$ of children worldwide being exposed (54). A study conducted in China in 2012 found that exposure of children to second hand smoke was associated with decreased lung function, with males being more affected than females (152). Lastly, a cross sectional study of data sets generated worldwide found that children exposed to second hand smoke exhibited decreased lung function (55).

Animal models have been used extensively to extend results of epidemiological studies in humans and allow investigation of disease mechanism. The oral model for murine nicotine exposure (as conducted) has been developed and widely used since 1983 (136). Using this model, investigators have not found decreased body weight or fluid intake due to nicotine supplementation in the drinking water (136). Most importantly, the amount of nicotine administered and absorbed into the blood stream in mice correlates to the amount of nicotine absorbed in moderate to severe human cigarette smokers (2-3 packs per day) (136). Our laboratory has previously published that using oral, transient, perinatal nicotine in mice $(100 \mu \mathrm{g} / \mathrm{mL})$ resulted in AHR through increased extracellular matrix production and altered lung branching morphogenesis $(42,64)$. The data presented within this chapter suggest that AHR is not sustained with aging for murine, transient nicotine (Figure 7) (reviewed in Table 4). In contrast, chronic nicotine exposure was found to cause long-standing lung functional abnormalities, which coincided with structural defects (Figure 7) (reviewed in Table 4). Rats showed similar finding to mice with perinatal 
nicotine causing enhanced methacholine-induced bronchoconstriction in which the disease state was inheritable through subsequent generations $(147,153)$. This transgenerational inheritance of asthma has also been shown positively associated in humans in a Norwegian cohort (154). In rhesus macaques perinatal nicotine exposure was determined to not only decrease lung function and cause alveolar hypoplasia, but also increased the expression of the $\alpha 7$ nicotine acetylcholine receptor (airway cartilage and vessels) and collagen deposition (large airways and vessels), which was in line with our previous findings in mice $(42,62-64)$. These publications are evidence that animal models are in line with human findings and may be used to further the understanding of OADs and their development.

Our studies show that nicotine exposure by itself, starting during embryogenesis and extending into adulthood, results in structural changes (characterized by enlarged alveolar spaces and increased collagen deposition) and abnormalities in lung function (increased compliance, decreased Newtonian resistance, and tissue elastance) in the adult lung. These changes were preceded by early alterations in alveolar size at 34-days after birth. Altogether, these observations may help explain the extensive body of epidemiological data linking tobacco smoke to the development of OAD in children, the mechanisms of which has continued to elude clinicians and researchers $(4,46,47,131)$. Furthermore, the observations presented add support to the notion that perinatal events followed by extended noxious exposures render the host susceptible to decreased lung function later in life. Although much work will be required to determine the mechanisms responsible for our observations, the following concepts should be considered. 
By focusing on nicotine, this and our prior reports point towards this agent (one of over 7000 components in tobacco smoke), as a major contributor to the long-standing effects of chronic tobacco smoke exposure on the mammalian lung $(42,64,155)$. They also provide further insight into the processes potentially disrupted by nicotine. For example, prior studies suggest that nicotine increases lung branching morphogenesis by increasing cleft formation and airway bifurcation. This, without comparable changes in lung growth, was suggested to promote dysanaptic lung growth, a term that describes independent alterations in branching and growth in developing lungs, but about which there is little mechanistic understanding. The current study also points to alveolarization as another potential developmental process targeted by nicotine in the mammalian lung. This is evident from our results generated at 34-days of age (which is at or close to the end of the alveolarization stage for mice), that shows chronic nicotine exposed mice have increased alveolar volume compared to untreated controls (Fig. 9). Previously, we showed that susceptibility to the effects of nicotine in the embryonic lung occurs during a tight temporal window, mainly between gestation day 14 and postnatal day 7 (42). This period overlaps with the latter half of the pseudoglandular stage of lung development largely characterized by branching morphogenesis (between 12 and 16.5 days of gestation in the mouse), the canalicular stage, during which vascularization occurs (between 16.5 and 17.5 days of gestation), the terminal sac stage (from 17.5 days of gestation to 4 days post birth), and the alveolar stage characterized by formation of secondary septa (septation) and formation of alveoli (from postnatal day 4 until day 36$)(156,157)$. Thus, any of the mechanisms involved in the processes responsible for these stages could be affected. However, the early detection of enlarged airspaces points to disruption of alveolarization 
leading to what is termed 'alveolar simplification'. These findings are consistent with those reported by others in non-human primates, sheep, and rats $(62,63,135,147,153$, $158,159)$.

The identification of alveolarization as a key process disrupted by nicotine points to potential genes or groups of genes being targeted. In rats, for example, nicotine decreases elastin production, a matrix molecule important for alveolarization (160). Other genes also involved in alveolarization and potentially affected by nicotine are vascular epithelial growth factor or VEGF and IGF1 $(161,162)$. The described changes in gene expression could be dependent on epigenetic modifications as previously demonstrated with perinatal nicotine exposure resulting in silencing of $\operatorname{PPAR} \gamma(163)$. Some of these genetic and demonstrated phenotypic changes in lung can be observed in the setting of intrauterine growth retardation (164). Considering the latter, we examined mouse weights (as a surrogate for lung size) at 34 and 210-days of age, but found no major differences. Unfortunately, we were unable to address lung growth or maturation at earlier time points.

We previously suggested that nicotine promotes alterations in lung branching by affecting the expression of fibronectin, a matrix glycoprotein implicated in branching morphogenesis (64). Above, we mentioned the role of elastin in alveolarization. Here, we show increased collagen deposition in the lungs of animals chronically exposed to nicotine, thereby further implicating aberrant extracellular matrix expression and turnover in control of lung development $(165,166)$. The detection of collagen mainly around airway structures points to effects on airway development, but further studies will be required to analyze this in more detail. 
It should be emphasized that the histological changes described above were associated with significant alterations in lung function later in life. Of these, the observed increase in compliance (suggesting decreased elastic recoil) is most intriguing as increased compliance is associated with tobacco smoke-related emphysema, a disease characterized by enlarged airspaces due to destruction of alveolar septa (167). In our model, the changes observed in the adult lung were apparent at 34-days of age suggesting abnormalities in alveolar simplification. This is more akin to "congenital lobar emphysema' although more widespread. However, the further increase in alveolar size in adult lungs (when compared to 34-day old lungs) suggests continuing alterations in lung structure; perhaps due to destruction of alveolar septa, but this requires further investigation.

Interestingly, animals exposed chronically to nicotine also showed decreased Newtonian resistance, a parameter considered to represent resistance of the large airways (114). The latter is not common in patients with tobacco smoke-related COPD or in animal models of emphysema, which typically show increased airway resistance (114). It is conceivable that this is related to the observed increase in collagen around the airways, which could theoretically make the airways rigid, thereby 'stenting' the airways open. However, this is pure speculation and further studies will be needed to investigate the implications of these observations.

As no major changes were detected in animals exposed to nicotine until day 7 after birth, additional exposure beyond this time is apparently required to produce the detrimental effects of nicotine exposure on lung histology and function that we observed following chronic treatment. Although the pre- and postnatal exposures tested here were limited to nicotine, one can consider the possibility that other developmental (e.g. 
prematurity and/or intrauterine growth retardation) and postnatal exposures (e.g. secondhand smoke, viral infections, and/or bacterial pneumonias) could have similar detrimental effects leading to COPD in the adult lung.

Our studies also suggest a differential effect of nicotine depending on sex. Nicotine-induced alterations in alveolar size and abnormal lung function were most prominent in males. This is intriguing considering previous evidence of greater deleterious effects of nicotine or second-hand smoke on lung function in males $(135,168)$. Other studies have shown similar differences in lung function, but did not examine the role of sex within their groups (114).

Finally, it is attractive to consider how our data may point to potential targets for intervention that might reduce the detrimental effects of nicotine in lung development and possibly decrease its impact on the development of COPD in the adult. In addition to genes involved in extracellular matrix generation and lung growth and maturation, among other processes, it is well known that nicotine acts by directly binding to cell surface nAChRs. In previous work, we showed that the effects of nicotine on lung branching morphogenesis ex vivo and on airway structure and function in vivo were mediated by $\alpha 7 \mathrm{nAChRs}$ since they were inhibited by antagonists to this receptor ex vivo, and were prevented in $\alpha 7$ nAChR knockout animals $(42,64)$. Since inhibitors of nAChRs are currently available commercially, it is tantalizing to think that safe and effective interventions against the detrimental effects of nicotine are within our reach.

In summary, we show that in contrast to transient nicotine exposure, chronic nicotine exposure starting during embryogenesis is detrimental to murine lung structure and function later in life, thereby rendering further support to the concept that perinatal and 
persistent exposures may impact the development of chronic lung disease in the adult. Although others have identified related abnormalities, to our knowledge this is the first data generated to confirm the detrimental effects of perinatal nicotine on lung structure and function long-term. 
CHAPTER III: A MURINE MODEL FOR EARLY LIFE INFLUENZA A H1N1

PANDEMIC 2009 INFECTION 


\section{$\underline{\text { Introduction }}$}

In vivo animal model systems for the study of respiratory tract viral infections have become essential for furthering our understanding of how viruses behave in humans, and for gaining insights into the host response to such infections. Unfortunately, in vivo models for the study of early life respiratory tract viral infections are limited or are rarely designed for the survival of the animal $(72,169-173)$. Consequently, there is scarcity of data regarding viral infections in young mammals, how the host responds acutely, and the implications of such events later in life $(46,67-70,174)$.

Common respiratory tract viral infections include, but are not limited to, influenza A virus (IAV), respiratory syncytial virus (RSV), rhinoviruses, coronaviruses, and adenoviruses. Of these viruses, rhinoviruses, RSV, and IAV are the most prominent viral respiratory pathogens affecting infants and young children throughout the globe, often causing severe lower respiratory tract infections; the latter not often seen in rhinoviruses $(68,169,175)$. Both RSV and IAV are similar in that they are enveloped negative-sense RNA viruses, elicit similar clinical symptoms, and may promote long-term abnormalities in lung physiology following infection during infancy $(68,174)$. Both viral entities represent a large proportion of cases for hospitalization worldwide, although in the U.S. RSV has 16 times higher incidence of hospitalization (compared to influenza) of children younger than one year of age, and influenza has 8 times more incidence for hospitalization for children older than five years of age $(176,177)$. Unlike RSV and IAV, rhinoviruses are positive-sense RNA viruses that typically do not replicate in the lower respiratory tract $(70,71) . \mathrm{RSV}$, IAV, and rhinoviruses are all known to cause exacerbations in persons with chronic OADs and all are associated with asthma later in life, when infection occurs during 
infancy $(72,174,175,178)$. Bisgard and colleagues analyzed epidemiological data and found that the risk factors associated with the development of OADs later in life due to a respiratory tract infection are susceptibility to infection (e.g. age and/or immunocompromised state) and the intensity of the response of the host to the infectious agent (bacteria or viral), rather than the agent itself (174).

Unfortunately, there is no effective vaccine against RSV or rhinoviruses, and influenza vaccination cannot occur until 6 months of age. This leaves a window where infants have no protection against influenza. Furthermore, vaccinated persons are still susceptible to new strains that arise through antigenic shift or drift as seen with the 2009 pandemic swine flu or the Spanish flu in 1918 (179). Together, these observations point to the importance of early life respiratory tract viral infections, not only because of their acute impact during critical stages of development, but also because of their potential implications later in life. However, the association between early life viral infection with chronic lung dysfunction in the adult has not yet been studied extensively.

The 2009 influenza pandemic was caused by a variant H1N1 human virus that arose due to a reassortment in swine and led to a variant termed pandemic H1N1 (pH1N1) (180). Early on, investigators observed increased pH1N1 infection in individuals between 10-19 years of age when compared to seasonal influenza ( $25.1 \%$ versus $15.6 \%$ of cases). A decreased incidence of $\mathrm{pH} 1 \mathrm{~N} 1$ infection was noted in very young individuals up to 4 years of age $(8.9 \%$ versus $14.6 \%)$ and in those over 65 years of age $(3.1 \%$ versus $12.1 \%)(181)$. Older individuals were determined to have protective immunity against pH1N1 due to immunologic encounters with viruses similar to pH1N1 earlier in life $(181,182)$. Therefore, the majority of cases infected with pH1N1 in 2009 occurred in adolescent 
individuals in whom a comparative murine model has yet to be developed and/or characterized. In this chapter, we measure respiratory function in mice at a developmental stage similar to that of humans around high school age, which represents the population with highest prevalence of pH1N1 infection in 2009. IAV was used due to it being a common infection that takes place in both upper and lower respiratory, throughout development into adulthood.

Typically, 8-12 week old mice are used for influenza infection and infection of younger mice is not as common $(79,183-188)$. Eight to twelve week old mice are known to be immune competent, whereas 14- and 30-day old mice may or may not have fully developed immune systems $(75,76,189-191)$. Data presented in this chapter provide evidence that mice at 14- and 30-days of age are at least partially immune competent. There are differences in the immune response (adaptive, innate, cellular, and humoral) between humans and mice (192). Although, the immune system is complex, 8-12 week old mice are generally immunocompetent, while in humans the development of the immune system is complete around or after twenty years of age (191). These ages of mice were chosen due to their lungs still being in a developmental stage (alveolarization) and due to their immature immune system $(156,157)$.

Here, we describe the development and characterization of a murine model of early life infection using a mouse adapted IAV pH1N1 strain. The age of the mice chosen for the model corresponded to the developmental stage (alveolarization) of the lung for individuals belonging to the most susceptible age group during the 2009 H1N1 pandemic (14 and 30 days of age). The model will serve as a tool to study early life infection of IAV 
pH1N1 which will be valuable for examining the pathogenesis and treatment of this condition.

$\underline{\text { Materials and Methods }}$

Cell Culture

Madin-Darby canine kidney (MDCK) cells (ATCC \#CCL-34 Manassas, Virginia) were used for virus propagation and titer determination assays. MDCK cells were cultured with “Complete Dulbecco’s Modified Eagle Medium” (CDMEM) (Table 4). MDCK cells were washed twice with Dulbecco's Phosphate Buffered Saline (DPBS) (Invitrogen \#14190-250 Carlsbad, California) and passaged using 0.25\% Trypsin EDTA (Invitrogen \#25200056). Trypsin EDTA was neutralized with CDMEM (formulation above) at a ratio of 1:5. MDCK and used until passage 30 .

\section{Virus Propagation}

A mouse adapted IAV California/07/2009 (ma-CA/07/09) was obtained from the University of Louisville Center for Predictive Medicine (Louisville, KY). Adaptation to the $\mathrm{C} 57 \mathrm{Bl} / 6$ murine line occurred through a series of 10 intranasal passages from the previous murine lung homogenate at $10 \% \mathrm{~W} / \mathrm{V}$ with DPBS. At the $10^{\text {th }}$ passage, the lung was homogenized and was then diluted 1:10 in Viral Growth Media (VGM) (Table 4) for passage over confluent MDCK cells. Viral supernatant was harvested upon the appearance of cytopathic effect and subsequently diluted 1:10 and passaged twice more over MDCK cells. Lastly, as a positive control for the in vitro assays, we obtained and propagated (as described above) IAV pH1N1, strain New York/18/2010 that was initially propagated in 
an embryonic hen egg from The University of Louisville Center for Predictive Medicine (Louisville, KY). Viral supernatants were aliquoted and then stored at $-80^{\circ} \mathrm{C}$ for short term use and $-120^{\circ} \mathrm{C}$ for long term storage. All stocks for experimental use were determined to be free of mycoplasma using a LookOut Mycoplasma PCR Detection Kit (Sigma \#MP0035).

Viral Titer Determination

$\mathrm{TCID}_{50} / \mathrm{mL}$ assays were performed on unaltered whole lung homogenate with MDCK cells seeded and infected as previously described $(193,194)$. Spot hemagglutination and plaque assays confirmed $\mathrm{TCID}_{50} / \mathrm{mL}$ assay results. Briefly, confluent MDCK cells were seeded into 6-well plates (Coring \#3506 Corning, New York) and incubated for 24 hours at $37^{\circ} \mathrm{C}, 5 \% \mathrm{CO}_{2}$. MDCK cells were infected as previously described and plates were incubated at $37^{\circ} \mathrm{C}, 5 \% \mathrm{CO}_{2}$ for 1 hour (193). After incubation, virus was removed from the plates, cells were washed twice, and a $2 \mathrm{~mL}$ volume of Complete Overlay Agar (Table 4).

After 96 hours of incubation $\left(37^{\circ} \mathrm{C}, 5 \% \mathrm{CO}_{2}\right.$ in a humidity chamber), $4 \%$ paraformaldehyde (Sigma \#158127) was added directly onto the agar at a volume of $2 \mathrm{~mL}$ for 20 minutes. Agar was then removed from the wells and $2 \mathrm{~mL}$ crystal violet staining solution was added for 20 minutes. Wells were washed twice with DPBS and plaques were subsequently counted for viral titer determination. 
Table 5. Media formulations

\begin{tabular}{|c|c|c|c|}
\hline $\begin{array}{c}\text { Formulation } \\
\text { Name }\end{array}$ & Reagent & $\begin{array}{c}\text { Vender Catalog } \\
\text { Number }\end{array}$ & Concentration \\
\hline \multicolumn{4}{|c|}{ Complete Dulbecco's Modified Eagle Medium } \\
\hline & $\begin{array}{l}\text { Dulbecco's Modified } \\
\text { Eagle Medium }\end{array}$ & Invitrogen \#11965 & $86.50 \%$ \\
\hline & Fetal Bovine Serum & Invitrogen \#16000044 & $10 \%$ \\
\hline & L-Glutamine & Invitrogen \#25030 & $5 \mathrm{mM} / 2.5 \%$ \\
\hline & Penicillin/Streptomycin & Invitrogen \#15140122 & $100 \mu \mathrm{g} / \mathrm{mL} / 1 \%$ \\
\hline Viral Growth & $\begin{array}{l}\text { Media } \\
\text { Dulbecco's Modified } \\
\text { Eagle Medium } \\
\end{array}$ & Invitrogen \#11965 & $91.24 \%$ \\
\hline & BSA Fraction V & Invitrogen \#15260-037 & $0.2 \% / 2.66 \%$ \\
\hline & L-Glutamine & Invitrogen $\# 25030$ & $5 \mathrm{mM} / 2.5 \%$ \\
\hline & Penicillin/Streptomycin & Invitrogen \#15140122 & $100 \mu \mathrm{g} / \mathrm{mL} / 1 \%$ \\
\hline & HEPES Buffer & Invitrogen \#15630-080 & $25 \mathrm{mM} / 2.5 \%$ \\
\hline & TPCK Trypsin & Sigma \#T8642 & $2 \mu \mathrm{g} / \mathrm{mL} / 0.1 \%$ \\
\hline \multicolumn{4}{|c|}{ Complete Overlay Agar } \\
\hline & Agar & Oxoid \#LP0011 & $0.80 \% / 38.7 \%$ \\
\hline & $\begin{array}{l}\text { Dulbecco's Modified } \\
\text { Eagle Medium }\end{array}$ & Invitrogen \#12100-061 & $1 \mathrm{x} / 50 \%$ \\
\hline & BSA Fraction V & Invitrogen \#15260-037 & $0.20 \% / 2.7 \%$ \\
\hline & L-Glutamine & Invitrogen \#25030 & $5 \mathrm{mM} / 4 \%$ \\
\hline & Penicillin/Streptomycin & Invitrogen \#15140122 & $100 \mu \mathrm{g} / \mathrm{mL} / 1 \%$ \\
\hline & HEPES Buffer & Invitrogen \#15630-080 & $25 \mathrm{mM} / 2.5 \%$ \\
\hline & $\begin{array}{l}\text { Non-Essential Amino } \\
\text { Acids }\end{array}$ & Invitrogen \#11140-050 & $1 \mathrm{x} / 1 \%$ \\
\hline & TPCK Trypsin & Sigma \#T8642 & $2 \mu \mathrm{g} / \mathrm{mL} / 0.1 \%$ \\
\hline
\end{tabular}


vRNA Isolation, cDNA Synthesis, and Hemagglutinin Sanger Sequencing

The ma-CA/07/09 MDCK passage 3 stock supernatant was used for characterization of the HA gene. Briefly, $0.75 \mathrm{~mL}$ of Trizol LS (Invitrogen \#10296010) was added separately to eight $0.25 \mathrm{~mL}$ volumes of the ma-CA/07/09 stock and processed according to the manufacturer's instructions. After the chloroform separation step, all upper phases from each individual tube were pooled. RNA was resuspended in $20 \mu \mathrm{L}$ RNase-Free water and stored at $-80^{\circ} \mathrm{C}$. One $\mu \mathrm{g}$ of RNA was used for cDNA synthesis using the iScript cDNA Synthesis Kit (Bio-Rad \#1708891 Hercules, California) according to the manufacturer's instructions. cDNA was aliquoted and stored undiluted at $-80^{\circ} \mathrm{C}$ or at $33 \mathrm{ng} / \mu \mathrm{L}$ at $-20^{\circ} \mathrm{C}$ for immediate use.

PfuUltra II Fusion Hot Start DNA Polymerase (Agilent \#600670 Santa Clara, California) was used for all sequencing PCR reactions. Briefly, a PCR master mix was created according to the manufacturer's instructions and added to 100 ng of RNA. Onethousand nucleotide length overlapping PCR fragments were amplified, spanning the entire HA gene, using the following primer sets: Forward\#1 ATGAAGGCAATACTAGTAGTT CTG, Reverse\#1 CTAGATTGAATAGACGGGATATTCC; Forward\#2 CCAAAGCTCA GCAAATCCTAC, Reverse\#2 GTCCCATTTTTGACACTTTCCATG; Forward\#3 GAA ATAGCAATAAGACCCAAAGTG, Reverse\#3 TTAAATACATATTCTACACTGTAG AGACC. PCR conditions followed the manufacturer's specifications. Gel electrophoresis was conducted on PCR reactions and bands of the expected length were excised then processed using the wizard SV Gel and PCR Clean-up System (Promega \#A9281 Fitchburg, Wisconsin) according to the manufacturer's directions. Purified PCR products were submitted for Sanger sequencing analysis at University of Louisville's Center for 
Genetics and Molecular Medicine DNA Facility Core. Two Sanger sequencing samples were submitted with each PCR amplicon using both the forward and reverse primers from each primer set. Sanger sequencing results were cataloged and assembled in CLC workbench.

\section{In Vivo Infections}

Mice were housed in a pathogen-free barrier facility accredited by the Association for Assessment and Accreditation of Laboratory Animal Care, and procedures were approved by the University of Louisville's Institutional Animal Care and Use Committee. Timed breeding was implemented by pairing C57BL/6 mice and un-pairing within 48 hours. At 14- or 30-days of age, mice were anesthetized and then they were subject to two intranasal inoculations of our in vivo propagated ma-CA/07/09 viral stock at $15 \mu \mathrm{L}$ (30day old mice) or $10 \mu \mathrm{L}$ (14-day old mice) volumes. Mice were weighed daily until 21 days post infection (DPI) at which time mouse blood was collected for serum using BD SST Microtainer Tubes (BD \#365967 Franklin Lakes, New Jersey), and the animals were given an intraperitoneal injection of sterile saline at $0.5 \mathrm{~mL}$ (GrowCells \#MSDW-2000).

\section{Euthanasia Criteria for Murine Infection}

Weight loss was the first criterion scored for all mice in the study (i.e. no mice showed any other sign of infection before weight loss). Due to our mice still maturing to adulthood (i.e. gaining weight due to growth), a typical $20-25 \%$ weight loss from time of infection for euthanasia criteria was not acceptable. Therefore, mock infected mice were weighed and determined the percent of weight gained from the previous 3 days. When an 
animal met weight loss criteria and scored 3 or greater in any of the categories listed, this dictated humane euthanasia (Table 5). For humane euthanasia, mice were anesthetized then cleaned with $70 \%$ ethanol and blood was collected via cardiac puncture. Cervical dislocation was then implemented to ensure humane euthanasia before dissection. 
Table 6. In vivo Infections Humane Endpoint Criteria

\begin{tabular}{|c|c|c|c|c|}
\hline \multicolumn{5}{|l|}{ Criterion } \\
\hline \multirow[t]{4}{*}{ Weight Loss } & \multicolumn{4}{|c|}{ Mouse is $75 \%$ body weight or less of expected weight gain } \\
\hline & \multicolumn{4}{|c|}{$\begin{array}{c}\text { When weight loss criterion above was reached a combined score of } 3 \text { or more } \\
\text { across all categories (below) dictated humane endpoint criteria }\end{array}$} \\
\hline & \multicolumn{4}{|c|}{ Score } \\
\hline & $\mathbf{0}$ & 1 & 2 & 3 \\
\hline Lethargy & Normal & $\begin{array}{c}\text { Mice are bunched } \\
\text { together with normal } \\
\text { movement }\end{array}$ & $\begin{array}{c}\text { Mice are bunched } \\
\text { together with slower } \\
\text { movement }\end{array}$ & $\begin{array}{l}\text { Mouse is alone and } \\
\text { shivering }\end{array}$ \\
\hline $\begin{array}{l}\text { Respiratory } \\
\text { Distress }\end{array}$ & Normal & $\begin{array}{l}\text { Up to } 25 \% \text { difference is } \\
\text { respiration compared to } \\
\text { uninfected mice }\end{array}$ & $\begin{array}{c}\text { Between } 25-50 \% \\
\text { difference is reparation } \\
\text { compared to uninfected } \\
\text { mice }\end{array}$ & $\begin{array}{l}\text { Above } 50 \% \\
\text { difference is } \\
\text { reparation compared } \\
\text { to uninfected mice }\end{array}$ \\
\hline $\begin{array}{l}\text { Physical } \\
\text { Signs of } \\
\text { Distress }\end{array}$ & Normal & $\begin{array}{l}\text { Slightly hunched } \\
\text { and/or 0-1 MGS score }\end{array}$ & $\begin{array}{l}\text { Pronounced hunching } \\
\text { and/or 1-2 MGS score }\end{array}$ & $\begin{array}{l}\text { Severe hunching } \\
\text { and/or } 2 \text { MGS score }\end{array}$ \\
\hline $\begin{array}{l}\text { Response to } \\
\text { Stimuli }\end{array}$ & Normal & $\begin{array}{l}\text { Slow response to } \\
\text { stimuli and normal } \\
\text { righting reflex }\end{array}$ & $\begin{array}{l}\text { Delayed response to } \\
\text { contact and normal } \\
\text { righting reflex }\end{array}$ & $\begin{array}{l}\text { Unresponsive to } \\
\text { contact and has no } \\
\text { righting reflex. }\end{array}$ \\
\hline
\end{tabular}




\section{IAV ELISA for Seroconversion}

Blood was processed for serum per the manufacturer's specifications, then aliquoted and stored at $-80^{\circ} \mathrm{C}$. Aliquoted serum was used for a qualitative mouse $\mathrm{H} 1 \mathrm{~N} 1$ Antibody IgG ELISA Kit (My BioSource \#MBS9350017) following the manufacturer's instructions.

\section{Tissue Processing}

Histology: The chest cavity was then opened and the inferior vena cava was cut. The lung vasculature was flushed by puncturing the right ventricle with a $0.26 \mathrm{G}$ needle and injecting $10 \mathrm{~mL}$ sterile saline solution. Lungs were inflated via the trachea with $10 \%$ neutral buffered formalin (VWR \#16004-128) at a constant pressure of $25 \mathrm{~cm} \mathrm{H} \mathrm{H}_{2} \mathrm{O}$. Inflated lungs were removed and placed in 10\% neutral buffered formalin for storage until separation of individual lobes then processed using a Spin Tissue Processor 120 (Thermo Fisher \#813150) according to the manufacturer's instructions $(42,64)$.

Lobes were then paraffin embedded and were then cut at a thickness of $6 \mu \mathrm{m}$ (Leica Jung \#RM2055) taking horizontal sections of all lobes. Slides were then stained using standard procedures for H\&E (Thermo Fisher \#6765007) (Thermo Fisher \#6766007) and Masson's trichrome (MTC) (American MasterTech \#KTMTR2PT). After staining tissue sections were dehydrated using standard procedures and then mounted with Vectamount mounting medium (Vector Laboratories \#H5000). Quantification of collagen was conducted using ImageJ (Version 1.51k) software as previously described $(139,140)$. 
Immunohistochemistry: Lung sections were cut and processed as above. Trilogy reagent (Cell Marque \#920P-10 Darmstadt, Germany) was used according to the instructions (Steps 1-12) for deparaffinization and rehydration. Slides were washed with Tris-buffered saline-Tween20 (0.05\%) for 1 minute, 4 times. Slides were blocked with $10 \%$ normal goat serum in wash buffer, for 1 hour at room temperature. Blocking reagent was removed and fibronectin primary antibody (Sigma \#F3648) was subsequently added, at 1:250 $(2 \mu \mathrm{g} / \mathrm{mL})$ for 1 hour at $37^{\circ} \mathrm{C}$ with appropriate $\operatorname{IgG}$ control (Sigma). Slides were washed and then incubated with $3 \% \mathrm{H}_{2} \mathrm{O}_{2}$ for 15 minutes at room temperature. Slides were washed and then incubated with a goat anti-rabbit horseradish peroxidase conjugated secondary antibody (Sigma \#9169) at 1:500 dilution for 30 minutes at room temperature. Slides were developed using the ImmPACT DAB Peroxidase Substrate Kit (Vector Laboratories \#SK4105 ) according to the manufacturer's instructions. As soon as slides developed they were immediately immersed in $\mathrm{dH}_{2} \mathrm{O}$ for 3 minutes. Tissue sections were dehydrated and coverslips were mounted as before.

\section{ImageJ Pixel Quantification}

Quantification of collagen and fibronectin was conducted using ImageJ (Version $1.51 \mathrm{k})$ software. Individual lung images were imported into ImageJ software in .tiff format and analyzed as previously described $(139,140)$. For our analysis, we applied a minimum threshold level of 37 and a maximum threshold level of 150 or 190 (14- or 30-day old mice) for our Masson's trichrome stained slides (collagen) and a minimum threshold level of 150 and a maximum threshold level of 220 was applied for our fibronectin stained slides. 


\section{Lung Viral Load Quantitation}

The chest cavity was opened and lungs were removed and placed into $2 \mathrm{~mL}$ microtubes containing $1.4 \mathrm{~mm}$ ceramic beads (Omni International \#19-627 Kennesaw, Georgia). Upon their removal, the lungs were immediately placed on ice and then homogenized with ice cold DPBS (10\%) W/V using a Fisher Bead Mill 4. Lung homogenate was centrifuged at $500 \mathrm{x}$ g for 5 minutes and supernatant was then aliquoted and stored at $-80^{\circ} \mathrm{C}$ for viral load determination via $\mathrm{TCID}_{50} / \mathrm{mL}$ and hemagglutination assays as described above.

\section{Lung RNA Isolation}

Lungs were removed and placed into $2 \mathrm{~mL}$ microtubes containing $1.4 \mathrm{~mm}$ ceramic beads containing $1 \mathrm{~mL}$ of Trizol reagent (Invitrogen \#15596026). Lungs were homogenized as before and RNA was isolated following the Trizol reagent protocol until completion of "Phase Separation" portion. The upper phase was then processed using the Directzole RNA Miniprep Plus Kit (Zymo Research \#R2072 Irvine, California) according to the manufacturer's instructions. RNA quantification was conducted as before and RNA was stored at $-80^{\circ} \mathrm{C}$ until use for cDNA synthesis/RT-PCR or a murine Affymetrix Clariom D microarray according to the manufacturer's instructions.

\section{Primary Lung Fibroblast Isolation, Infection, and RNA Isolation}

Primary lung fibroblasts from C57BL/6 mice were isolated as previously described $(196,197)$. Fibroblasts (passage 9) were seeded onto 6-well plates at 500,000 cells/well in CDMEM for 24 hours. Cells were washed twice and then infected with our in vitro 
propagated ma-CA/07/09 viral stock in duplicate. Cells were infected at a multiplicity of infection (MOI) of 1.0 at a volume of $100 \mu \mathrm{L}(\mathrm{VGM})$ for 1 hour at $37^{\circ} \mathrm{C}, 5 \% \mathrm{CO}_{2}$. After viral incubation virus was removed and cell monolayers were washed as before then supplemented with $2 \mathrm{~mL}$ of VGM per well. At 48 hours post infection (HPI) supernatant was removed and cell monolayers RNA was collected using Trizol reagent. RNA was isolated as described above. One $\mu \mathrm{g}$ of RNA was used for cDNA synthesis using the iScript cDNA Synthesis Kit according to the manufacturer's instructions.

\section{Transcriptome Assay (Clariom D Microarray)}

Briefly, biotinylated cRNA was prepared from total RNA using Affymetrix GeneChip Whole Transcript Plus Reagent kit. Fragmented cRNA was hybridized to Affymetrix mouse Clariom D arrays and processed on an Affymetrix FS-450 fluidics station and scanned on an Affymetrix GeneChip scanner. The resulting .cel files were imported into Partek Genomics Suite 6.6 (6.15.0327) and transcripts were normalized and summarized using RMA default settings. Partek was used for generation of the Principal Component Analysis (PCA) and heat map. Our generated microarray data was uploaded into Clarivate Analytics (formerly Thomson Reuters) Metacore analysis program found online. A $+/-2$ - fold and $0.1 \geq q$-value (step-up p-value) cut-off was applied to our data set (as before) and our 81 differentially expressed genes were categorized into the most implicated pathway maps, and process networks as well as gene ontology (GO) groups (with the most significant results listed). 
$R T-P C R$

$100 \mathrm{ng}$ of cDNA per well was loaded into 96-well PCR plate (Thermo Fisher \#4366932) in triplicate for each primer/probe set being tested. For each treatment, we had duplicate samples for primer/probe sets: IL-1 $\beta \quad\left(\mathrm{mm} 00434228 \_\mathrm{m} 1\right)$, TGF $\beta$ (mm01178820_m1), MMP9 (mm00442991), Collagen 1a1 (mm00801666_g1), fibronectin (mm01256744_m1), and $\beta$-actin (mm04394036_g1) for our housekeeping control gene. Furthermore, non-template controls or $1 \mu \mathrm{L}$ of RNA from each sample as well as water was tested in duplicated for each gene. TaqMan fast advanced master mix (Thermo Fisher \#4444557) was used and the reactions were conducted in a StepOne Plus machine (Applied Biosystems \#4376600 Foster City, California) following the manufacturer's conditions.

\section{Murine Lung Function Testing}

Untreated mice $(\mathrm{n}=19 ;$ male $=10$, female $=9)$, H1N1 ELISA positive mice $\left(10^{1}\right.$ TCID $50 / \mathrm{mL}$ per mouse: $\mathrm{N}=13,6$ males, 7 females; $10^{1.7} \mathrm{TCID}_{50} / \mathrm{mL}$ per mouse: $\mathrm{N}=19$, 9 males, 10 females) at 7 months of age were subject to functional lung testing. Pulmonary function and response to increasing concentrations of methacholine $(0-50 \mathrm{mg} / \mathrm{mL}$, inhaled $)$ were measured by forced oscillation using the flexiVent system (SCIREQ, Montreal, Quebec, Canada) as previously described $(137,138)$.

\section{Statistics}

Using GraphPad Prism 7.03 the following statistical test were implemented. For lung viral load determination all challenges were compared independently using a Logrank 
(Mantel-Cox test) as well as a Gehan-Breslow-Wilcoxon test separately. The average daily weights were calculated and a percent weight gain along with standard deviations from time of infection and were compared using a two-way ANOVA analysis along with a Turkey multiple comparison test. The percent of total lung area stained (normalized to IgG controls for immunohistochemistry, entire lung) for each group were averaged and a oneway ANOVA analysis was conducted with all groups using a Bonferroni test to correct for multiple comparisons. RT-PCR data for each individual samples $\Delta \Delta \mathrm{Ct}$ value was used in the formula, $2^{-\Delta \Delta \mathrm{Ct}}$ and differences between groups were determined using unpaired T-tests with a Holm-Sidak test to correct for multiple comparisons.

Using SAS statistical software, a correlation chart between all six outcome parameters (resistance, compliance, elastance, Newtonian resistance, tissue damping, and tissue elastance) was generated and showed strong correlation for all parameters to resistance (Supplemental Fig. 1). A 3-factor ANOVA was then implemented with the factors representing experimental group, sex type, and dose of methacholine elicited. Descriptive statistics were calculated for the entire study population as well as within each factor. Rigorous analysis involved the main effects for 3-factor ANOVA along with 2-way interactions (group*sex, group*dose, sex*dose). Since interaction effects were not significant in most of the outcome measures (only exception was for compliance in the sex*dose interaction), we considered only 3-factor ANOVA without interaction terms. Rather than subset analysis, we have used contrast to test the main effects (such as mock versus $10^{1} \mathrm{pH} 1 \mathrm{~N} 1$; mock versus $10^{1.7} \mathrm{pH} 1 \mathrm{~N} 1$, and $10^{1} \mathrm{pH} 1 \mathrm{~N} 1$ versus $10^{1.7} \mathrm{pH} 1 \mathrm{~N} 1$ ), which increases power to detect significant effects $(143,144)$. 


\section{$\underline{\text { Results }}$}

\section{Propagation and Characterization of Viral Stock}

We propagated IAV pH1N1 isolates using MDCK cells over three passages collecting at 3 DPI for all ma-CA/07/09 stocks and at 7 DPI for our in vitro positive control stock, New York/18/2009. The concentration of our ma-CA/07/09 viral stocks for both in vivo and in vitro studies was determined to be above $1 \times 10^{8}$ via $\mathrm{TCID}_{50} / \mathrm{mL}$ and plaque assays with standard deviations at or below $10^{+/-0.22}$ (Table 6). 
Table 7. Viral Stock Titers

\begin{tabular}{|c|c|c|c|c|}
\hline Virus Stock & $\begin{array}{c}\text { TCID } 50 / m L \\
\log _{10}\end{array}$ & SD & $\begin{array}{c}\text { PFU/mL } \\
\log _{10}\end{array}$ & SD \\
\hline $\begin{array}{l}\text { Mouse Adapted CA/07/2009 } \\
\text { MDCK P2 }\end{array}$ & 7.28 & $\begin{array}{l}0.21 \\
\mathrm{~N}=3\end{array}$ & N.A. & $\begin{array}{l}\text { N.A. } \\
\mathrm{N}=0\end{array}$ \\
\hline $\begin{array}{l}\text { Mouse Adapted CA/07/2009 } \\
\text { MDCK P3, In Vivo }\end{array}$ & 8.25 & $\begin{array}{l}0.17 \\
\mathrm{~N}=4\end{array}$ & 8.34 & $\begin{array}{l}0.20 \\
\mathrm{~N}=5\end{array}$ \\
\hline $\begin{array}{l}\text { Mouse Adapted CA/07/2009 } \\
\text { MDCK P3, In Vitro }\end{array}$ & 8.04 & $\begin{array}{l}0.22 \\
\mathrm{~N}=3\end{array}$ & 8.03 & $\begin{array}{l}\text { N.A. } \\
N=1\end{array}$ \\
\hline $\begin{array}{l}\text { New York/18/2009 } \\
\text { MDCK P1 }\end{array}$ & 4.80 & $\begin{array}{l}0.21 \\
\mathrm{~N}=6\end{array}$ & 4.87 & $\begin{array}{l}0.10 \\
N=3\end{array}$ \\
\hline
\end{tabular}


Once the propagation of ma-CA/07/09 was deemed successful, hemagglutinin (HA) gene PCR products derived from our viral stock were sequenced to determine host receptor binding preference. When comparing our viral stock (GenBank \#MF988319) to the original human isolate (GenBank \#KU933485.1), we found two nucleotide (A $\rightarrow \mathrm{G})$ mutations at positions 460 and 665 in the HA gene (data not shown). These two nucleotide changes resulted in two amino acid mutations at sites $154(\mathrm{~K} \rightarrow \mathrm{E})$ and $222(\mathrm{D} \rightarrow \mathrm{G})($ data not shown). The glycine substitution at position 222 confirmed that the murine adaptation of our virus was successful and the propagation over 3 MDCK cell passages did not affect the binding phenotype of our stock (185).

In vivo Replication Kinetics of Mice Infected at 14-Days of Age

Fourteen-day old mice were infected intranasally with the propagated ma$\mathrm{CA} / 07 / 09$ in vivo stock for lung viral load quantification with challenges ranging from $10^{1}$ $10^{1.7}$ ma-CA/07/09 viruses as determined from $\mathrm{TCID}_{50} / \mathrm{mL}$ titer (in two $10 \mu \mathrm{L}$ volumes). We found that the peak viral load was challenge-dependent and the time when the lung viral load peaked in challenges occurred at 3-4 days post infection (DPI) (Fig. 12A). The challenges delivered to mice corresponded to different peak viral loads in the lung with $10^{1.7}$ viruses of ma-CA/07/09 per mouse reaching a peak lung viral load of $1 \times 10^{7.310+/ 0.352}$ $\mathrm{TCID}_{50} / \mathrm{mL}$, whereas the lower challenges reached peak viral loads of $10^{5.980+/ 0.607}$ and $10^{5.800+/-0.816} \mathrm{TCID}_{50} / \mathrm{mL}$. At our lowest challenge ( $10^{1} \mathrm{ma}-\mathrm{CA} / 07 / 09$ viruses per mouse), one mouse had undetectable viral loads in the lung at both 3,4 and 9 DPI ( $80 \%$ detectable virus); these mice were not included in Figure 12A and B. No virus was detected in the lungs of mice for any challenge at 21 DPI. Furthermore, one mouse had undetectable viral 
load loads (as determined by the $\mathrm{TCID}_{50} / \mathrm{mL}$ assay) at 9 DPI for our middle challenge of $10^{1.3}$ ma-CA/07/09 viruses per mouse $(93.3 \%$ detectable virus as determined by the $\mathrm{TCID}_{50} / \mathrm{mL}$ assay). All mice infected with $10^{1.7} \mathrm{ma}-\mathrm{CA} / 07 / 09$ viruses per mouse were found to have virus as determined by the $\mathrm{TCID}_{50} / \mathrm{mL}$ (100\% detectable virus). Mice infected with $10^{1}$ ma-CA/07/09 viruses per mouse showed statistically different lung viral load titers at 3 DPI compared to $10^{1.3}(\mathrm{p}<0.05)$ and $10^{1.7} \mathrm{ma}-\mathrm{CA} / 07 / 09$ viruses per mouse $(\mathrm{p}<0.01)$. Lastly, $10^{1.7}$ ma-CA/07/09 viruses per mouse challenges had statistically different lung viral load titers at 4 DPI compared to $10^{1}(\mathrm{p}<0.05)$ and $10^{1.3} \mathrm{ma}-\mathrm{CA} / 07 / 09$ viruses per mouse $(\mathrm{p}<0.05)$ (Fig. 12A).

A survival graph was generated based on when mice reached humane endpoint criteria due to ma-CA/07/09 infection (Table 5). For all challenges, only mice in the $10^{1.3}$ $(\mathrm{N}=1)$ and $10^{1.7}(\mathrm{~N}=7)$ ma-CA/07/09 viruses per mouse reached humane endpoint criteria (Fig. 12B). It was determined that there were statistically significant differences in survival $(\mathrm{p}<0.05)$ between $10^{1.7}$ ma-CA/07/09 viruses per mouse groups and both other challenges.

Mice infected with ma-CA/07/09 showed statistical significant differences (compared to mock $\mathrm{N}=17$ ) in percent change in body weight starting at 5 DPI for $10^{1.7}$ maCA/07/09 viruses per mouse $(\mathrm{p}<0.01)$, and at 6 DPI for $10^{1}$ and $10^{1.3} \mathrm{TCID}_{50} / \mathrm{mL}$ per mouse $(\mathrm{p}<0.05)($ Fig. 12C). As mentioned earlier, the mock infected group showed weight gain over time, which was found to be statistically different from $10^{1}, 10^{1.3}$, and $10^{1.7}$ ma-CA/07/09 viruses per mouse at 7, 8, and 9 DPI $(\mathrm{p}<0.001)($ Fig. 12C). 
A
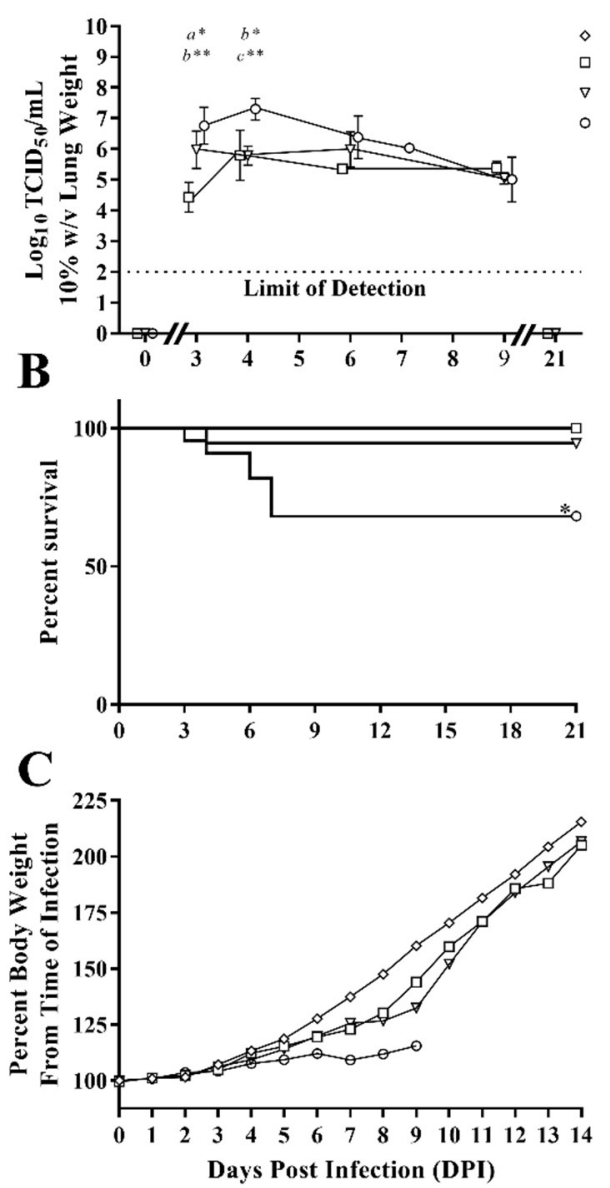

D

30 Days of Age

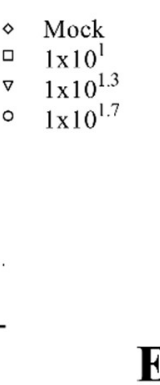

$\mathbf{E}$
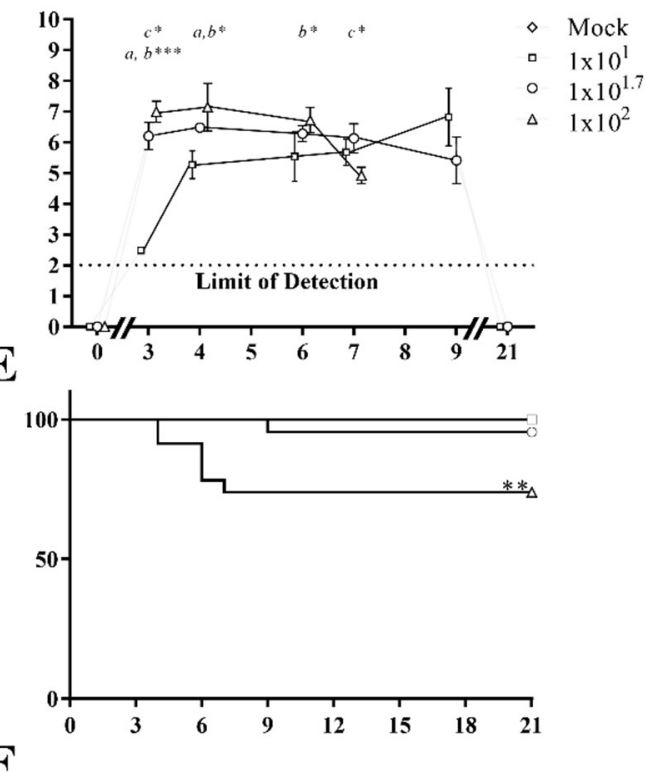

$\mathbf{F}$

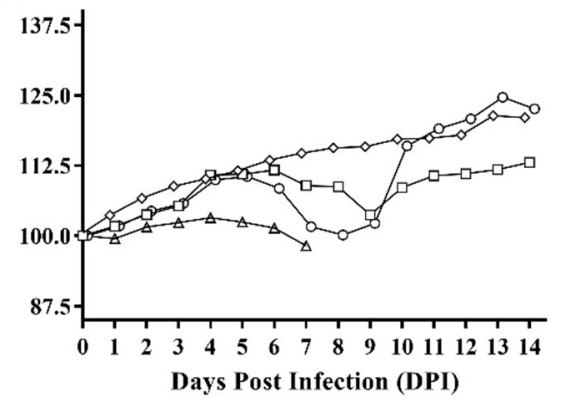

Figure 12. Lung Viral Load, Survival, and Weight Change in 14 and 30-Day Old

\section{C57Bl/6 Infection with ma-CA/07/09}

14 and 30-day old $\mathrm{C} 57 \mathrm{Bl} / 6$ mice intranasal infection with ma-CA/07/09 at the indicated challenges. Each individual mouse lung homogenate was independently titered three times via $\mathrm{TCID}_{50} / \mathrm{mL}$ assay with a minimum of 3 mice at each time point. Data represents the mean of all independent viral titers and their corresponding standard deviations. 14-day old mice lung viral load determination (A) $a: 10^{1}$ vs. $10^{1.3}, b: 10^{1}$ vs. $10^{1.7}, c: 10^{1.3}$ vs. $10^{1.7}$; and 30 -day old mice (D) $a: 10^{1}$ vs. $10^{1.7}, b: 10^{1}$ vs. $10^{2}, c: 10^{1.7}$ vs. $10^{2} ; \mathrm{p}<0.05: *$, $\mathrm{p}<0.01: * *, \mathrm{p}<0.001$ : $^{* *}$. Error bars correspond to standard deviation. The average viral load in the lung was determined by averaging each individual animal's log lung viral load (minimum 3 replicates) and then determining the mean log lung viral load at each day among individual mice (minimum 3 mice per day). The resulting data was imported into GraphPad Prism 7.03 and groups were compared using multiple unpaired, 
t-tests with p-values reported for each comparison. Corresponding survival curve of lung viral load determination for 14-day old mice (B) and 30-day old mice (E) p $<0.05: *, p<0.01$ : **. All challenges were compared independently using a Logrank (Mantel-Cox test) as well as a Gehan-Breslow-Wilcoxon test separately in GraphPad Prism 7.03. Corresponding weights of mice represented in $\mathbf{A}$ and $\mathbf{D}$ as the percent weight from the initial time of infection at the indicated challenges for 14-day old mice (C) and 30-day old mice (F). The average daily weights were calculated and a percent weight gain along with standard deviations from time of infection was calculated and imported into GraphPad Prism 7.03. Groups percent weight gained were compared using a two-way ANOVA analysis along with a Turkey multiple comparison test. Multiplicity adjusted p-values were reported for each comparison. 
In vivo Replication Kinetics of Mice Infected at 30-Days of Age

Thirty-day old mice were infected intranasally with the propagated ma-CA/07/09 in vivo stock for lung viral load quantification. Three challenges were chosen ranging from $10^{1-2}$ ma-CA/07/09 viruses as determined by the $\mathrm{TCID}_{50} / \mathrm{mL}$ titration (in two $15 \mu \mathrm{L}$ volumes). We found the peak viral load to be quite consistent across challenges, whereas time of peak viral load varied (Fig. 12D). As expected, the infections were challengedependent as the highest challenge ( $10^{1.7}$ and $10^{2}$ ma-CA/07/09 viruses per mouse) had their highest viral load at 4 DPI $\left(10^{6.480+/-0.136}\right.$ and $\left.10^{7.148+/-0.778} \mathrm{TCID}_{50} / \mathrm{mL}\right)$, whereas the lowest challenge $\left(10^{1} \mathrm{ma}-\mathrm{CA} / 07 / 09\right.$ viruses per mouse $)$ peaked at 9 DPI $\left(10^{6.818+/-0.936}\right.$ $\mathrm{TCID}_{50} / \mathrm{mL}$ ) (Fig. 12D). No virus was detected in the lungs of mice for any challenge at 21 DPI. At our lowest challenge ( $10^{1} \mathrm{ma}-\mathrm{CA} / 07 / 09$ viruses per mouse), one mouse had undetectable viral loads in the lung at both 3 and 6 DPI, and two mice had undetectable viral loads at 7 DPI ( $80 \%$ detectable virus); these mice were not included in Figure 12D and E. All mice infected with $10^{1.7}$ and $10^{2}$ ma-CA/07/09 viruses per mouse were found to have virus ( $100 \%$ detectable virus). $10^{1}$ and $10^{1.7} \mathrm{TCID}_{50} / \mathrm{mL}$ per mouse challenges resulted in statistically different lung viral load titers at $3(\mathrm{p}<0.01)$ and 4 DPI $(\mathrm{p}<0.05)$. Challenges of $10^{1.7}$ and $10^{2}$ ma-CA/07/09 viruses per mouse resulted in statistically different lung viral load titers at 3 and 7 DPI $(p<0.05)($ Fig. 12D). For all challenges, only mice in the $10^{2}(\mathrm{~N}=6)$ and $10^{1.7}(\mathrm{~N}=1)$ ma-CA/07/09 viruses per mouse reached humane endpoint criteria (Fig. 12E). It was determined that there were statistically significant differences in survival $(\mathrm{p}<0.01)$ between $10^{2}$ ma-CA/07/09 viruses per mouse challenge versus the other two challenges (Fig. 12E). 
Mice infected with ma-CA/07/09 showed a challenge-dependent decrease in percent change in body weight from time of infection starting at 5 DPI for $10^{2} \mathrm{TCID}_{50} / \mathrm{mL}$ per mouse, at 6 DPI for $10^{1.7} \mathrm{TCID}{ }_{50} / \mathrm{mL}$ per mouse, and at 7 DPI for $10^{1} \mathrm{TCID}_{50} / \mathrm{mL}$ per mouse (Fig. 12F). As mentioned earlier, the mock infected group showed weight gain over time, which was found to be statistically different from $10^{1} \mathrm{TCID}_{50} / \mathrm{mL}$ per mouse at 7 and 9 DPI $(\mathrm{p}<0.001)$. Furthermore, mock mice were found to be statistically different from $10^{1.7} \mathrm{TCID}_{50} / \mathrm{mL}$ per mouse at $6,7,8$, and 9 DPI $(\mathrm{p}<0.001)$. Lastly, mock infected mice were found to be statistically different from mice infected with $10^{2} \mathrm{TCID}_{50} / \mathrm{mL}$ per mouse at $2(\mathrm{p}<0.05), 3(\mathrm{p}<0.01), 4,5,6$, and 7 DPI $(\mathrm{p}<0.001)($ Fig. 12F).

\section{Lung Histological Changes in Infected Mice}

Fourteen-day and 30-day old mice were infected intranasally with our propagated ma-CA/07/09 in vivo stock for 4 days, euthanized, and then lung tissue harvested for staining (H\&E, Masson's trichrome, and IHC for fibronectin). These studies revealed challenge-dependent increases in collagen deposition, inflammation, and cell infiltration to areas around the large proximal airways.

H\&E staining of 14-day old mice, at 4 DPI, showed minimal differences between mock and $10^{1} \mathrm{TCID}_{50} / \mathrm{mL}$ per mouse groups. Comparing mice infected with $10^{1.3} \mathrm{ma}-$ $\mathrm{CA} / 07 / 09$ viruses (as determined by the $\mathrm{TCID}_{50} / \mathrm{mL}$ assay) per mouse to mock infected animals, we discovered mild inflammation in proximity to $\sim 15 \%$ of the large airways), mild cell infiltration (macrophages and lymphocytes) to areas only around the large proximal airways, and slight epithelial desquamation with necrotic epithelium in the lumen (where viral replication was taking place). Likewise, $10^{1.7} \mathrm{ma}-\mathrm{CA} / 07 / 09$ viruses per mouse 
challenge showed the same findings except all findings were moderate to severe inflammation (in proximity to $\sim 30 \%$ of the large airways) (Fig. 13Ai-iv). ImageJ analysis of slides stained with Masson's trichrome revealed a significant increase in collagen deposition around the large proximal airways in the most lethal challenge, $10^{1.7} \mathrm{ma}-$ CA/07/09 viruses per mouse $(\mathrm{p}<0.05)$, but not in the two lower sub-lethal challenges (Fig. 13Bi-iv and G). Evaluation for fibronectin resulted in no differences between mock infected and pH1N1 infected animals (Fig. 13Ci-iv and I).

For 30-day old mice, at 4 DPI H\&E staining revealed little to no differences between mock and $10^{1}$ ma-CA/07/09 viruses per mouse groups. When comparing $10^{1.7}$ $\mathrm{TCID}_{50} / \mathrm{mL}$ per mouse to mock infected, there was slight epithelial desquamation with necrotic epithelium in the lumen, and mild inflammation in areas around the large proximal airways (in proximity to $\sim 20 \%$ of the large airways). Likewise, when comparing $10^{2}$ ma$\mathrm{CA} / 07 / 09$ viruses per mouse, there was more pronounced (compared to $10^{1.7} \mathrm{ma}-\mathrm{CA} / 07 / 09$ viruses per mouse) epithelial desquamation with more necrotic epithelium in the lumen, inflammation (in proximity to $\sim 30-40 \%$ of the large airways), but not as evident in the parenchyma as seen in 14-day old mice) and cell infiltration (macrophages and lymphocytes) to areas around the large proximal airways and blood vessels (Fig. 13Di-iv). Similar to the above, increasing challenges of ma-CA/07/09 resulted in increased staining for collagen, around the large proximal airways close to the hilum (Fig. 13Ei-iv). Masson's trichrome stained slides showed a significant increase in collagen deposition between mock versus $10^{1.7}(\mathrm{p}<0.05)$ and $10^{2}(\mathrm{p}<0.01)$ ma-CA/07/09 viruses per mouse groups (in proximity to $\sim 30 \%$ of the large airways for both challenges) while no difference was found for stained collagen between mock versus $10^{1} \mathrm{TCID}_{50} / \mathrm{mL}$ per mouse groups $(\mathbf{F i g .} \mathbf{1 3 H})$. 
As in 14-day old mice, fibronectin IHC staining in 30-day old mice at 4 DPI showed no differences between mock infected vs $10^{1}$ or $10^{1.7} \mathrm{ma}-\mathrm{CA} / 07 / 09$ viruses per mouse groups although, versus $10^{2}$ ma-CA/07/09 viruses per mouse group there was significant increased staining $(\boldsymbol{p}<0.05)($ Fig. 13Fi-iv and $\mathbf{J})$. 


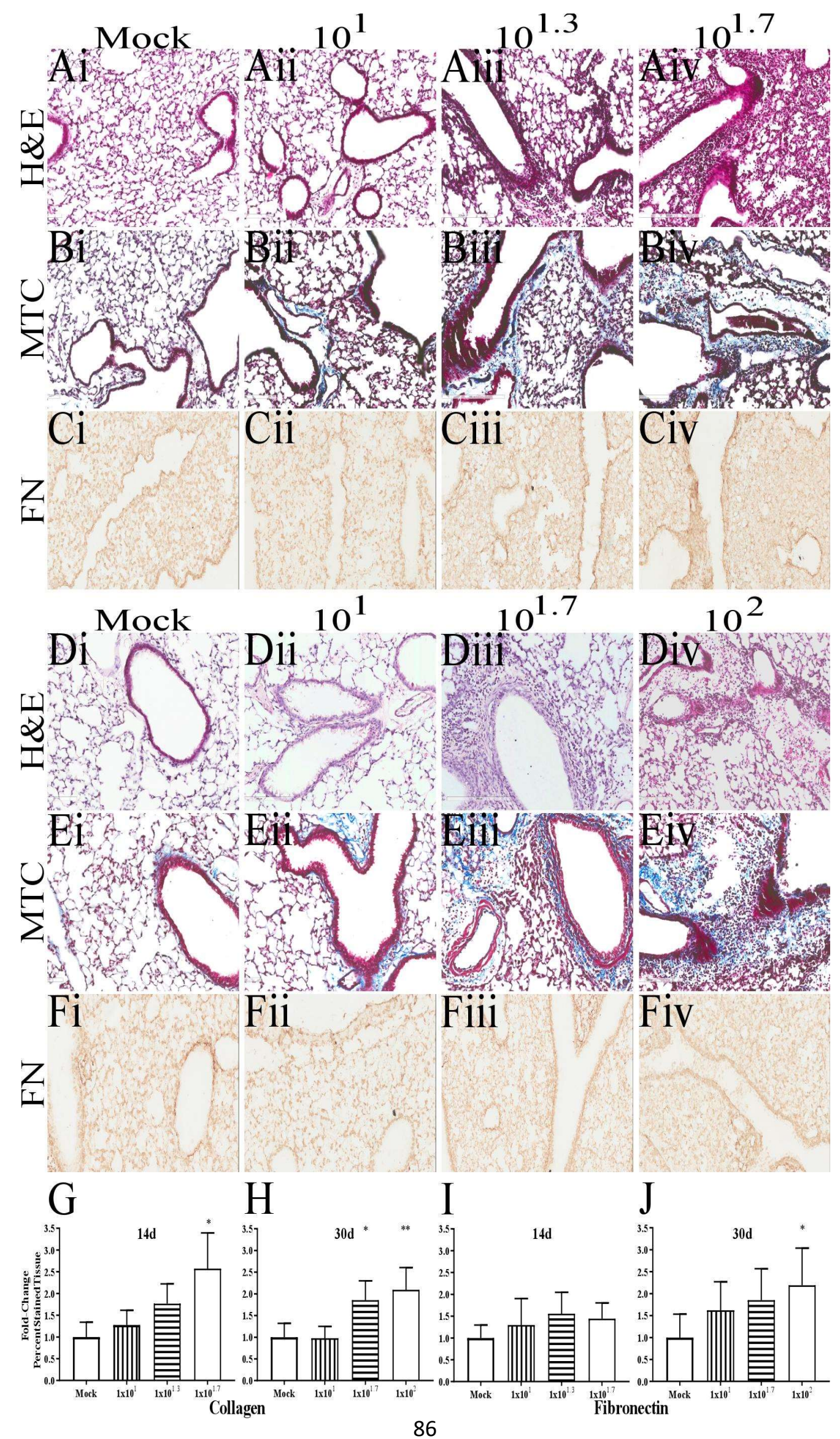




\section{Figure 13. Lung Histology of ma-CA/07/09 Infected Mice at 34-Days of Age}

14 and 30-day C57Bl/6 mice intranasal infection with ma-CA/07/09 at the indicated challenges. Fourteenday old mice H\&E staining at 10x (Ai-iv), Mason's Trichrome staining at 10x (Bi-iv), and Fibronectin immunohistochemistry at 10x (Ci-iv). Thirty-day old mice H\&E staining (Di-iv), Mason's trichrome staining (Ei-iv), and fibronectin immunohistochemistry (F-iv). Quantification for collagen represented as fold-change compared to mock infected controls, using Mason's Trichrome staining for 14-day old mice (G) and 30-day old mice $(\mathbf{H})$. Quantification for fibronectin represented as fold-change compared to mock infected controls, for 14-day old mice (I) and 30-day old mice (J). Differences are represented vs. mock

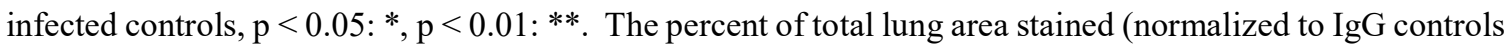
for immunohistochemistry, entire lung) for each group were averaged and imported into GraphPad Prism 7.03. One-way ANOVA analyses were conducted with all groups using a Bonferroni test to correct for multiple comparisons. Multiplicity adjusted p-values were reported for each comparison. Error bars correspond to standard error of the mean. 


\section{Whole Lung Global Transcript Profile in Infected Mice}

Considering the significant tissue remodeling observed in 30-day old mice, we decided to pursue further characterization of animals at this age. Genome-wide gene expression patterns were analyzed using whole lung extract at 4 DPI (Fig. 14). A PCA was conducted on all gene transcripts in the microarray after normalization and showed distinct separation between groups with tight groupings among biological replicates (Fig. 14A). The microarray resulted in the ma-CA/07/09-infected group having 253 genes with fold changes greater than 2, and 22 genes with fold changes less than -2, compared to the mock infected group. Furthermore, there were 77 genes with fold changes greater than 2 and 4 genes with fold changes less than -2 , when a 0.1 or less q-value cutoff was applied, which can be visualized in the heat map generated (Fig. 14B). We used MetaCore online software (Thomson Reuters), to unveil the most implicated GO for our restricted data set (Fig. 14B and C). The most implicated GO for cellular process resulted in the following top three hits: "Defense Response to Virus", "Innate Immune Response" and "Defense Response" ( $\mathrm{p}$-values $\leq 1 \times 10^{-11}$ ). The most implicated GO pathway maps resulted in the following top three hits: Interferon (IFN) $\alpha / \beta$ signaling via JAK/STAT, IFN $\alpha / \beta$ signaling via MAPKs, and Antiviral Actions of IFNs ( $\mathrm{p}$-values $\leq 1 \times 10^{-14}$ ). Lastly, the most implicated GO Process Networks resulted in the following top three hits: IFN signaling, Innate

Immune Response to RNA Viral Infection, and IFN $\gamma$ signaling (p-values $\left.\leq 1 \times 10^{-11}\right)\left(1 \times 10^{-4}\right.$ IFN $\gamma)($ Fig. 14C). 

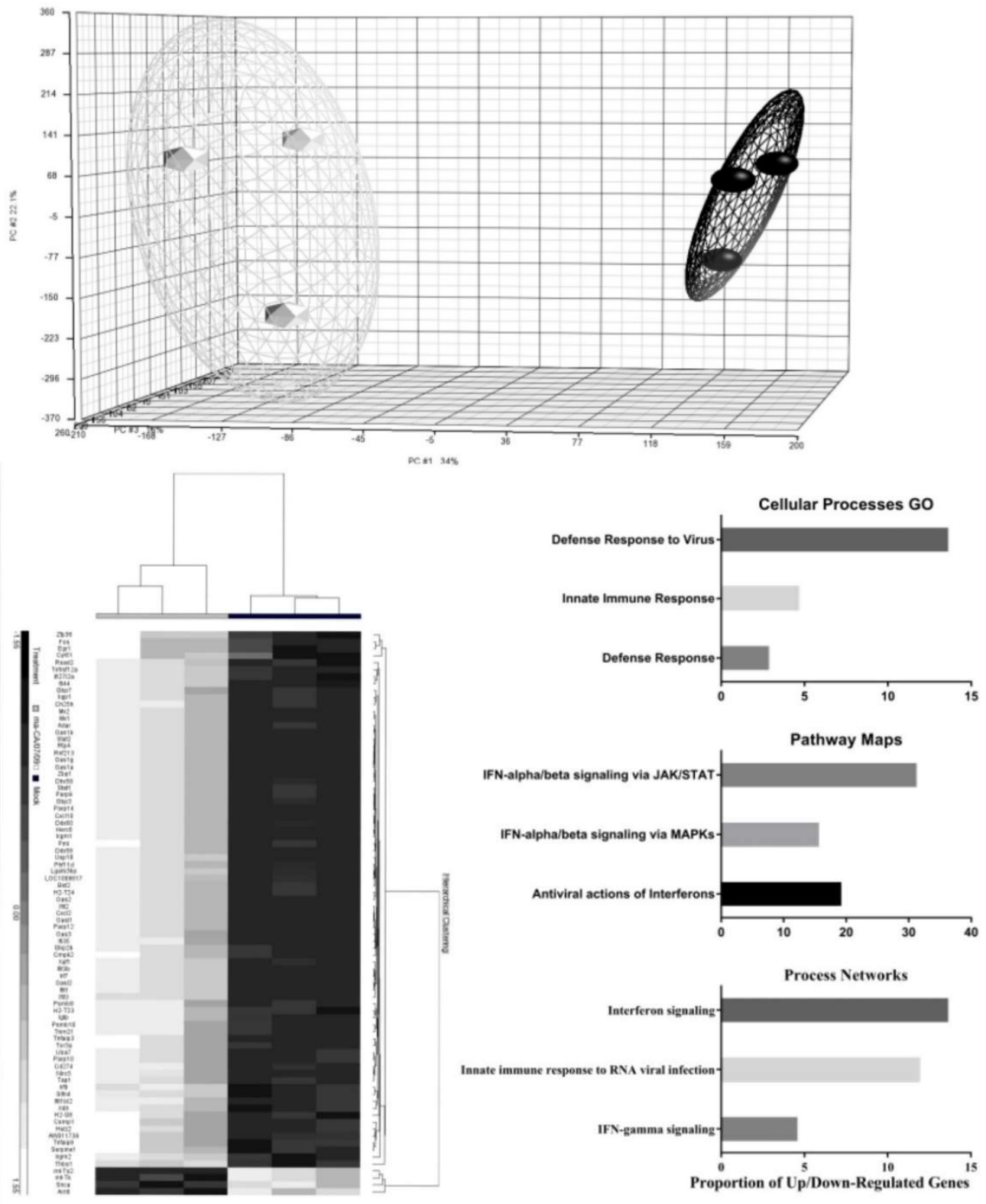

Figure 14. Infection of 30-Day Old Mice with ma-CA/07/09 Microarray at 34-Days

\section{of Age}

Clariom D microarray conducted with 3 mice per group (pH1N1 vs. uninfected). Samples were compared using a one-way ANOVA model. A step-up false discovery rate was applied as multiple test correction for the resulting q-values. Principal Component Analysis from all genes in Clariom D microarray (A). Heat 
Map from Clariom D microarray, with a $+/$ - 2-fold change and $\mathrm{q} \leq 0.1$ cutoff $(\mathbf{B})$. Percentage of updownregulated genes as seen in "B" according to GO, pathway map, and process networks (C). 
The most differentially expressed genes from our microarray (Fig. 14B) are listed in Table 7. These genes are all involved in IAV recognition and immune response associated with IAV infection. As can be seen, the most differentially expressed genes in the table are involved with IFN signaling pathways, which is known to be associated with influenza infections, and correlate to the most implicated GO (Fig. 14C).

Virus-Induced Tissue Remodeling Gene Expression in Primary Lung Fibroblasts

Our laboratory has studied the mechanisms responsible for lung tissue remodeling occurring after an insult. Many of our findings have been attributed to the production of extracellular matrix components by lung fibroblasts $(198,199)$. We therefore were interested in determining whether lung fibroblasts could play a role in the tissue remodeling seen in lungs harvested from infected animals (Fig. 13). We found at 48 HPI that primary lung fibroblasts (isolated from a mouse less than 3 months of age) infected in vitro with ma-CA/07/09 had significantly increased mRNA levels for tissue remodeling genes collagen 1a1, fibronectin, matrix metallopeptidase 9 (MMP9), and transforming growth factor beta (TGF $\beta$ ) compared to mock infected cells (Fig. 15). Further, viral titers in the supernatant from these cells was approximately $1 \times 10^{2.3}$ as determined from the $\mathrm{TCID}_{50} / \mathrm{mL}$ assay (data not shown). 


\section{Table 8. Microarray Most Differentially Expressed Genes (pH1N1 vs. Uninfected)}

\begin{tabular}{|c|c|c|c|c|}
\hline Gene & $\begin{array}{c}\text { Fold } \\
\text { Change }\end{array}$ & $\begin{array}{c}\text { Q - } \\
\text { Value }\end{array}$ & Function & $\begin{array}{l}\text { Probe Set ID } \\
\text { TC(X).mm.1 }\end{array}$ \\
\hline Cxcl10 & 16.15 & 0.077 & $\begin{array}{l}\text {-Induced by IFN } \gamma(200) \\
\text {-Produced by human alveolar macrophages in } \\
\text { response to H1N1 }(77,201) \\
\text {-Chemotactic for monocytes, NK \& T cells (202) }\end{array}$ & 0500002756 \\
\hline Slfn4 & 15.19 & 0.094 & $\begin{array}{l}\text {-Induced by Type I IFN receptor activation (203) } \\
\text {-Produced by murine type II airway epithelial } \\
\text { cells (204) } \\
\text {-Macrophage activation and myelopoiesis (205) }\end{array}$ & 1100001241 \\
\hline Mx1 & 11.15 & 0.085 & $\begin{array}{l}\text {-Induced by type I \& III IFN (206) } \\
\text {-Cellular IAV antiviral response (207) } \\
\text {-Inhibits IAV protein PB2-NP interaction (206) }\end{array}$ & 1600002148 \\
\hline Ifit3b & 10.29 & 0.067 & $\begin{array}{l}\text {-Induced by type I \& III IFN via Jak/Stat pathway } \\
(208) \\
\text {-Produced by primary human macrophages }(209) \\
\text {-Inhibits IAV endosome fusion }(210,211) \\
\end{array}$ & 1900000502 \\
\hline Ifit1 & 10.13 & 0.071 & $\begin{array}{l}\text {-Induced by IFN and/or viral infection (212) } \\
\text {-Found to not be antiviral as previously thought, } \\
\text { in KO C57B1/6 mice or human cells (no 5'-ppp } \\
\text { interaction) (212) }\end{array}$ & 1900000504 \\
\hline Irf7 & 9.69 & 0.074 & $\begin{array}{l}\text {-Produced by plasmacytoid dendritic cells in } \\
\text { large amounts in response to virus }(213) \\
\text {-Regulatory factor/activates IFN } \alpha / \beta \text { and adaptive } \\
\text { immune response }(214) \\
\text {-Human and murine survival factor }(214,215)\end{array}$ & 0700004530 \\
\hline Usp18 & 8.34 & 0.073 & $\begin{array}{l}\text {-Negative regulator of type I IFN signaling } \\
\text { (ISG15) (216) } \\
\text {-ISG15-specific deubiquitinating protease (217) } \\
\text {-Expression levels correlated to survival in } \\
\text { chickens infected with IAV (218) } \\
\end{array}$ & 0600001369 \\
\hline Ifit3 & 8.27 & 0.066 & $\begin{array}{l}\text {-Induced by type I \& III IFN via Jak/Stat Pathway } \\
\text { (208) } \\
\text {-Produced by primary human macrophages (209) } \\
\text {-Inhibits IAV endosome fusion }(210,211)\end{array}$ & 1900000501 \\
\hline $\mathrm{Zbp} 1$ & 8.25 & 0.084 & $\begin{array}{l}\text {-Induced by IFN (219) } \\
\text {-KO murine mortality reduced via reduced } \\
\text { inflammation (NLRP3 pathway) and epithelial } \\
\text { damage (219) } \\
\text {-IAV Rig-I sensing leads to ZbP1 ubiquitination } \\
\text { causing cell death (220) }\end{array}$ & 0200005290 \\
\hline Rsad2 & 7.87 & 0.098 & $\begin{array}{l}\text {-Highly inducible by type I \& II IFN (221) } \\
\text {-Inhibits IAV release (222) }\end{array}$ & 1200001552 \\
\hline mt-Tk & -3.50 & 0.03108 & -No known association to IAV infection & $0 \mathrm{M} 00000008$ \\
\hline mt-Ts2 & -2.76 & 0.02460 & -No known association to IAV infection & $0 \mathrm{M} 00000015$ \\
\hline Snca & -2.42 & 0.05225 & -No known association to IAV infection & 060000239 \\
\hline Arntl & -2.19 & 0.02460 & $\begin{array}{l}\text {-Core gene involved in circadian rhythm (223) } \\
\text {-KO mice showed increased survival, decreased } \\
\text { airway resistance, and increased compliance after } \\
\text { IAV infection (224) }\end{array}$ & 0700001660 \\
\hline
\end{tabular}




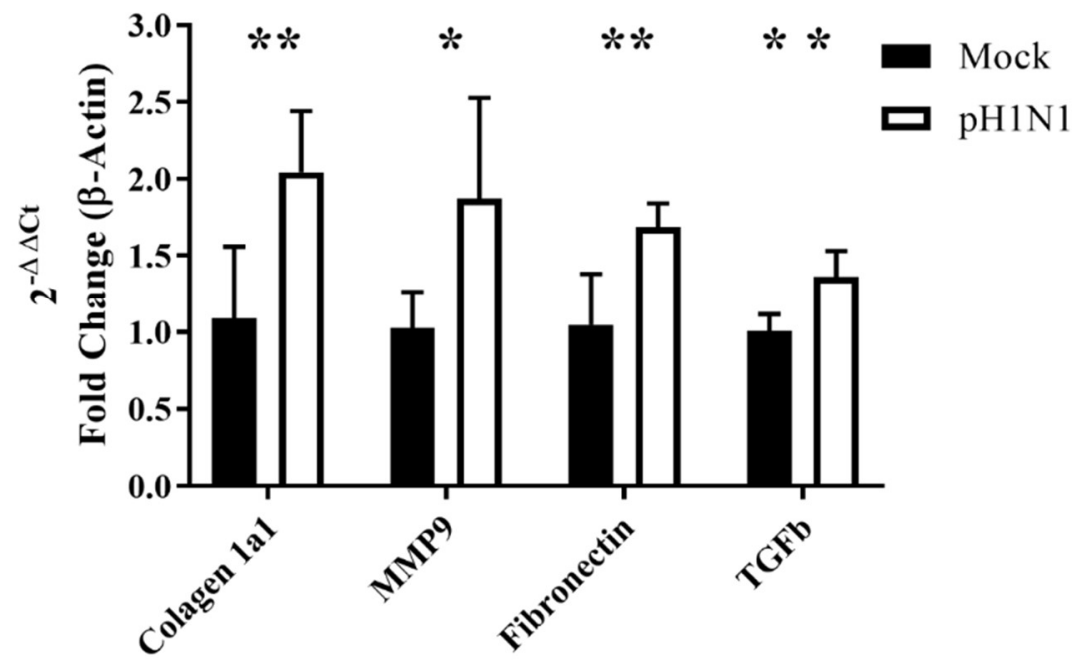

Figure 15. ma-CA/07/09 Infected Primary Lung Fibroblast Altered RT-PCR Tissue

\section{Remodeling Genes}

TaqMan RT-PCR of ma-CA/07/09 infected C57Bl/6 primary lung fibroblasts at $48 \mathrm{HPI}$, represented as foldchange compared to mock infected controls. $\mathrm{p}<0.05: *, \mathrm{p}<0.01$ * $^{*}$. Ct values for genes of interest were normalized to $\beta$ actin $(\Delta \mathrm{Ct})$ and then $\Delta \Delta \mathrm{Ct}$ values were determined by normalizing to the average mock $\Delta \mathrm{Ct}$ values. Each individual samples $\Delta \Delta \mathrm{Ct}$ value was used in the formula, $2^{-\Delta \Delta \mathrm{Ct}}$ and imported into GraphPad Prism 7.03. Differences between groups were determined using multiple unpaired T-tests. P-values were reported for each comparison with error bars represent standard deviations. 


\section{Pulmonary Function Testing}

Having evaluated the development of inflammation and tissue remodeling after infection, we wondered if these early events could lead to functional abnormalities in the adult lung. For this, we examined lung function at 210-days age in mice that had been infected at 30-days of age with $10^{1}$ and $10^{1.7}$ ma-CA/07/09 viruses per mouse. Resistance, compliance, elastance, Newtonian resistance, tissue damping, and tissue elastance were examined at baseline and after stimulation with increasing concentrations of methacholine from $0-50 \mathrm{mg} / \mathrm{mL}$. For statistical analysis we only evaluated responses at baseline, 25, and $50 \mathrm{mg} / \mathrm{mL}$ of methacholine. We first determined that a strong correlation existed between the outcome parameters tested, with exception of compliance (Fig. 16). In Figure 17, we present data related to resistance, tissue damping, and tissue elastance showing significant differences between mock versus $10^{1.7}$ ma-CA/07/09 viruses per mouse group $(p=0.041$, $\mathrm{p}=0.024, \mathrm{p}=0.048$ ) (summarized in Table 9). In addition, we found differences when comparing parameters at baseline versus those generated after methacholine stimulation, but not between the two doses of methacholine. Interestingly, we discovered that most differences identified were related to changes in males suggesting differences between sexes (Fig. 17). 


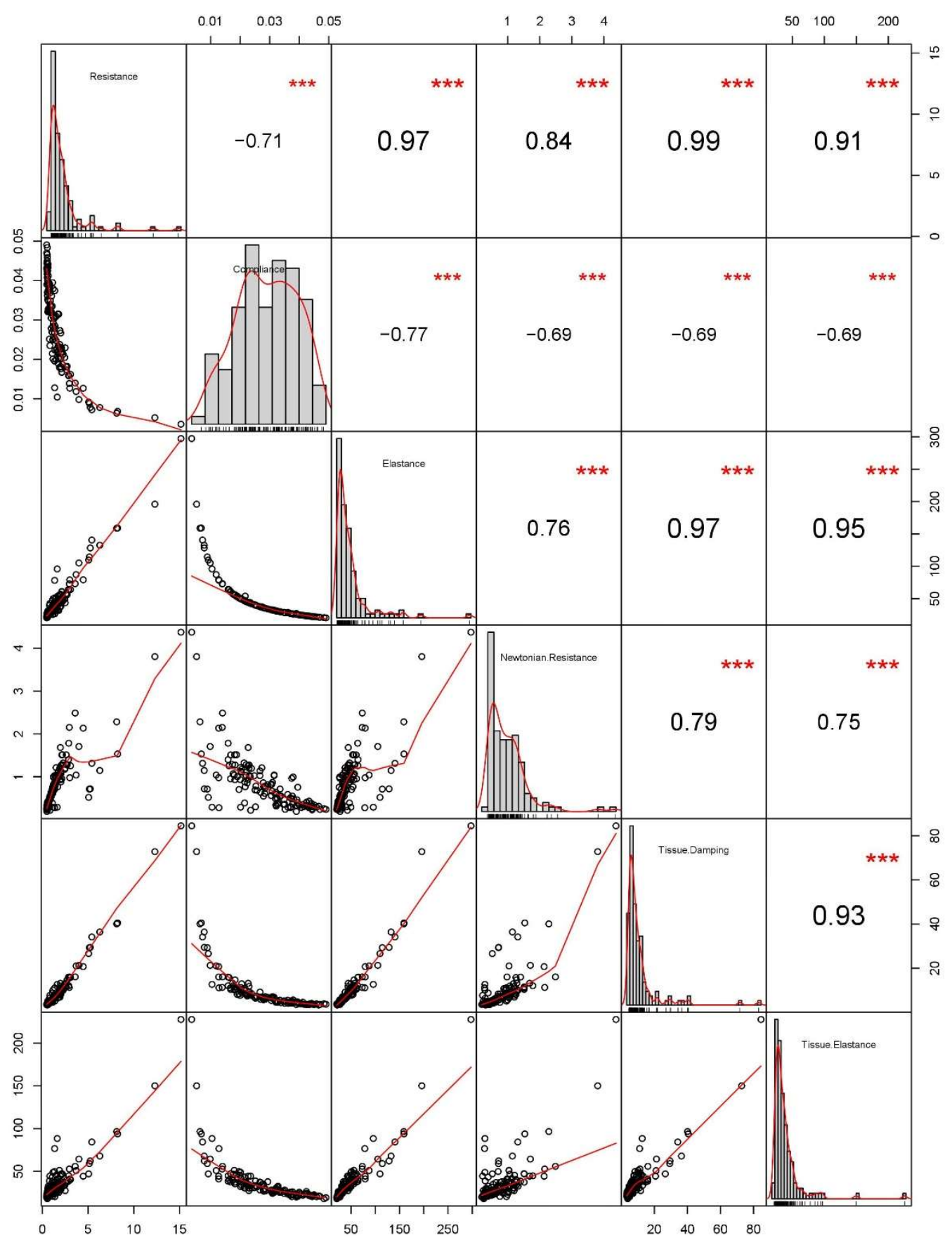

Figure 16. Correlation of Pulmonary Function Test Outcome Parameters for pH1N1 Statistical Analysis (All Groups)

All data used for analysis in SAS 3 Factor ANOVA. Graphical representation of correlation represented in bottom left of figure. Fit of data to linear line of regression represented in top right of figure. 


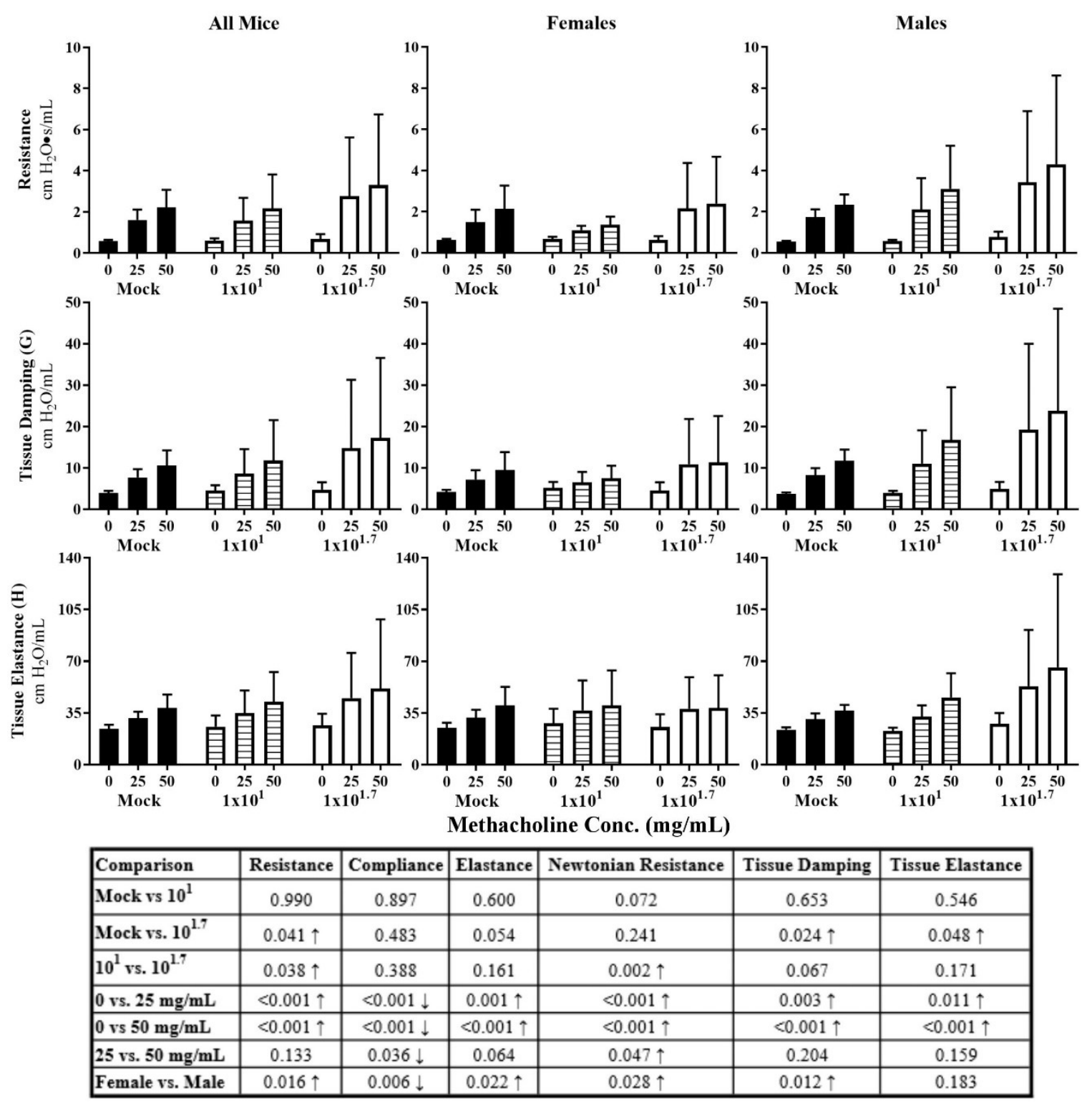

Figure 17. Pulmonary Function Test Results for ma-CA/07/09 Infection in 30-Day

\section{Old Mice at 210-Days of Age}

C57B1/6 mice infected with ma-CA/07/09 or DPBS at 30-days of age were assessed at 210-days of age using a SCIREQ FlexiVent machine, at increasing concentrations of methacholine $(0-50 \mathrm{mg} / \mathrm{mL})$. Averages of results were taken for each individual mouse where the COD was greater than or equal to $95 \%$. SAS statistical software was used to conduct 3-factor ANOVA without interaction terms. Results were declared significant at $5 \%$ significance level $(\mathrm{p}<0.05)$. Error bars represent standard deviation from the mean. P-values are 
represented in the table with significant results having an arrow dictating the direction of the second group in each row compared to the first group. 


\section{Table 9. Summary of pH1N1 Pulmonary Function Testing}

\begin{tabular}{|c|c|c|c|c|c|c|}
\hline \multicolumn{2}{|c}{ Resistance } & Compliance & Elastance & $\begin{array}{c}\text { Newtonian } \\
\text { Resistance }\end{array}$ & $\begin{array}{c}\text { Tissue } \\
\text { Damping }\end{array}$ & $\begin{array}{c}\text { Tissue } \\
\text { Elastance }\end{array}$ \\
\hline pH1N1 & $\uparrow$ & No Change & $\begin{array}{c}\text { No } \\
\text { Change }\end{array}$ & No Change & $\uparrow$ & 个 \\
\hline
\end{tabular}

Arrows indicate direction of parameter compared to untreated-mock infected control Blue indicates alteration in function driven by males

Black indicates alteration in function not driven by either sex 


\section{Discussion}

To date there are a limited number of publications on early life or neonatal murine influenza infection aimed for the survival of the animal. Herein, we show the lung viral load detected after 3 challenges of pH1N1 in two different aged C57BL/6 mice groups and corresponding lung histology, total mRNA microarray at the time of peak lung viral load, and the assessment of lung function long term. We believe this model is highly relevant to individuals infected during the 2009 Swine Flu pandemic.

IAV was chosen due to its ability to infect humans during the alveolarization period of lung development. RSV infections also occur during the alveolarization period, but primarily occurs soon after birth. IAV represents a less severe infection for humans and thus we would predict, based on the severity and the time of infection, that these experiments would provide insight on how other viruses act on lung development. On the other hand, rhinoviruses primarily have been proven to only infect the upper respiratory tract, unlike IAV and RSV, which may not cause functional alterations discovered by using IAV (specifically, tissue damping and tissue elastance) $(67,71,225)$.

The IAV strain used in the study was originally responsible for the 2009 influenza pandemic, that began in April, in the southwestern United States. The CA/07/09 isolate was originally isolated on April $9^{\text {th }}, 2009$ from California and represents an ancestral strain from the pandemic outbreak; it was used for vaccination purposes worldwide due to the phenotype similarities to all other pH1N1 isolates $(226,227)$. IAV, H1N1 in humans primarily infects the upper respiratory tract through $\alpha 2-6$ sialic acid receptors on transmembrane glycoproteins (primarily located in the upper airway) $(171,185,228)$. The differences in receptor binding specificity observed for the H1N1 strains are mapped to a 
single amino acid residue (222) in the HA protein, with binding to the $\alpha 2-6$ sialic acid receptors restricted to an aspartic acid residue and $\alpha 2-3$ sialic acid receptor binding conferred by a glycine residue (185). Thus, having a glycine at the 222 amino acid decreases pathogenesis of influenza in humans. For $\mathrm{pH} 1 \mathrm{~N} 1$, inherent replication errors of the viral genome lead to variant viral progeny in which some possess the phenotype that confers $\alpha 2-3$ sialic acid receptor binding (quasispecies) (229). Mice primarily express $\alpha 2-$ 3 sialic acid receptors throughout their entire respiratory tract (171). Therefore, to conduct research in murine models with IAV H1N1 isolated from humans, the virus must first be adapted to the murine species in order to increase the number of viral progeny containing the $\alpha 2-3$ sialic acid binding phenotype within the quasispecies population (185).

Our ma-CA/07/09 stock was subject to Sanger sequencing analysis of the HA gene. We found that our MDCK propagation of ma-CA/07/09 did not alter binding preference of the major population of the viral quasispecies in our stock. Furthermore, when determining the ma-CA/07/09 viral titer, low standard deviations resulted in both the $\mathrm{TCID}_{50} / \mathrm{mL}$ assay and plaque assay, with minimal variation between methods (Table 6). Having low standard deviations in our stock titers for experimental use allowed for a more accurate calculation of viral dilutions so dosing was consistent over the course of all infection experiments. In addition, when conducting experiments with negative sense viral agents, such as influenza, the concern of propagation of defective interfering (DI) particles arises. Due to propagating our viral stocks in MDCK cells, as opposed to hen eggs, along with similar titers between our $\mathrm{TCID}_{50} / \mathrm{mL}$ and plaque assays, we were confident that $\mathrm{DI}$ particles were not present (or represented a miniscule amount) in our quasispecies IAV stocks $(230,231)$. 
Previous studies with young animals involving viral infection administered a certain challenge corresponding to the mouse's weight $(72,173)$. We decided to deliver one single and consistent challenge to all animals in our infected groups. Our reasoning for this was that lethality due to $\mathrm{pH} 1 \mathrm{~N} 1$ does not appear to be based on the challenge delivered (when there is minimal difference between challenges), but the development of the immune response (both innate and adaptive). In addition, a subject's developmental stage may not correspond to body weight, especially when multiple mothers are used for the breeding of the mice $(72,74,76,173,232)$.

Following influenza infection of different mouse strains, there are differences in lethality that correlate with the extent of inflammatory response $(171,188,233,234)$. Furthermore, $\mathrm{pH} 1 \mathrm{~N} 1$ infection in mice shows major differences when compared to humans in that mice become hypothermic and the tropism of infections are different $(171,185,235$, 236). Recognizing the potential differences between a mouse model for IAV infection and a human model for IAV infection, we chose the C57BL/6 model based on our previous experience and the relative resistance of this inbred mouse strain to lethal effects of IAV, H1N1 compared to other strains $(42,64,183,188,233,234)$.

Challenges ranging from $1 \times 10^{1-2}$ ma-CA/07/09 viruses per mouse were administered to 14- and 30-day old mice and showed similar lung peak viral loads of approximately $1 \times 10^{7} \mathrm{TCID}_{50} / \mathrm{mL}$. Pulmonary viral replication kinetics were similar following all ma-CA/07/09 challenges in 14-day old mice, with peak viral loads at 3-4 DPI (Fig. 12A). Interestingly, viral loads following infection of 30-day old mice the lowest challenge ( $1 \times 10^{1}$ ma-CA/07/09 viruses per mouse) peaked at 9 DPI $\left(1 \times 10^{7}\right.$ ma-CA/07/09 viruses per mouse). This suggests that the virus may initially be replicating slowly in an 
area where the innate immune response is not fully developed such as the nasopharynx (232). The two higher challenges in 30-day old mice had peak replication in the lung at 4 DPI as expected (Fig. 12D). Survival and percent change in body weight from time of infection were dependent on the challenge delivered (Fig. 12). Peak viral loads quantitated in the lung of these animals were similar to, if not increased, compared to standardly used 8-12 week old mice and increased compared to 2-day old C57BL/6 mice intranasally infected with H1N1 $(173,183,186,233,234)$.

The pathology of the lung was examined at 4 DPI using H\&E, Masson's trichrome, and fibronectin immunohistochemistry. Staining revealed a challenge-dependent increase in immune cell infiltration, tissue remodeling (collagen and fibronectin), and inflammation with regions around the large airways being most affected (Fig. 13). Masson's trichrome staining analysis revealed that in 14-day old mice only the highest challenge caused tissue remodeling during the acute phase of infection. By contrast, in 30-day old mice, both the lethal challenge and the sub-lethal challenge resulted in increased collagen deposition compared to mock infected controls (Fig. 13 G\&H). These findings are consistent with what is reported in the literature, in that $\mathrm{H} 1 \mathrm{~N} 1$ promotes collagen and fibronectin deposition via SMAD signaling pathways that involve $\alpha \mathrm{V} \beta 6$ integrin-mediated TGF $\beta$ activation in human bronchial epithelial cells and in C57BL/6 mice (237). Immunohistochemistry for fibronectin revealed no differences between groups for 14-day old mice, whereas, in 30-day old mice at 4 DPI only the lethal challenge caused significant fibronectin deposition throughout the lung parenchyma and around the large airways (Fig. $13 \mathbf{I} \& \mathbf{J})$. The analysis was limited due to the use of an antibody against plasma fibronectin 
rather than cellular fibronectin, the latter being capable of detecting fibronectin-producing cells as opposed to total fibronectin.

Further characterization was done in the 30-day old mice model. The murine lung at this age is in or near completion of the alveolar stage of development (late fetal to 17-21 years postnatal in humans). According to the literature, lung maturation in mice is complete by or soon after 36 days post-birth, which correlates to 17-21 years of age in humans $(156,157)$. Therefore, using 30-day old mice correlates to the lung development stage of the group of individuals (high school aged) who had increased incidence of 2009, pH1N1 infection (181). Lastly, by using a 30-day old murine model we would predict that alterations in both function and structure would be more severe if infection occurred earlier in life (i.e. earlier in the alveolarization period of development).

PCA of gene expression data in sub-lethally infected $\left(1 \times 10^{1.7}\right.$ ma-CA/07/09 viruses per mouse) 30-day old mice versus mock infected controls showed distinct separation between groups and tight groupings of individual replicates (Fig. 14A). As expected, differences between groups in the array were mainly attributed to IFN signaling pathways associated with the defense against viral infection (Fig. 14C). What is notable is that both innate immune response signaling and seroconversion (serum tested via IAV ELISA qualitatively, data not shown) in these young animals were occurring (seroconversion also occurred in 14-day old mice but levels were noticeably lower). To our knowledge, this is the first publication of a microarray analysis of young C57BL/6 mice infected with pH1N1.

We then decided to further investigate the cause of tissue remodeling seen in infected mice. The RNA samples for the microarray were subject to TaqMan RT-PCR for key tissue remodeling genes, and, similar to the microarray results, no differences were 
seen (data not shown); this might have been attributed to dilution of transcripts from the isolated affected areas when using the entire lung. As an alternative approach, we investigated C57BL/6 primary lung fibroblasts, cells which our lab in the past has associated with increased extracellular matrix synthesis in response to insult. Using RTPCR, infected primary lung fibroblasts at 48 HPI were found to have increased MMP9, TGF $\beta$, collagen 1a1, and fibronectin expression compared to mock infected controls (Fig. 15). This finding implicates the primary lung fibroblasts as one mediator between viral replication and/or viral innate and/or adaptive signaling, to increased collagen and fibronectin deposition in the lung. It is known that MMP9 can activate TGF $\beta$ from its latent bound state to trigger SMAD pathway signaling resulting in the deposition of collagen, fibronectin, and other ECM components $(198,199,237)$. It is important to note that fibronectin has been published to be required for IAV H1N1, $\alpha$ 2- 6 sialic acid binding in human cell lines, but the context in the mouse is unknown (238).

After characterizing our model of infection during the acute stage, we were interested in determining if early life infection could lead to lung abnormalities later in life. Mice at 30-days of age were infected and allowed to age until 210-days of age, at which time they were subjected to lung functional testing. We found that early life pH1N1 challenge (at a sublethal dose) was enough to alter lung function with age and most importantly differences among sex type, with males being more affected than females (Fig. 17). Although we used a pH1N1 strain of influenza, we do not believe that the functional and structural alterations were $\mathrm{pH} 1 \mathrm{~N} 1$-specific, as seasonal influenza and other viruses could result in similar findings (if they occur during the alveolarization stage of lung development). Consistent with this, an epidemiological study found that the risk factors 
associated for the development of OADs later in life due to a respiratory tract infection are susceptibility to infection (e.g. age and/or immunocompromised state) and the intensity of the response of the host to the infectious agent (bacteria or viral), rather than the agent itself (174). The purpose of using pH1N1 was to provide a viral infection model relevant to humans after infancy.

It has been established in humans that females infected with influenza have a heightened risk for morbidity and mortality compared to males (239). In other work, mice have been published to have the same sex-driven differences in mortality and weight loss $(183,240)$. For our data, male mice showed significantly less weight loss (compared to starting weight at time of infection) than females after pH1N1 challenge (data not shown). This suggests that the weight loss due to infection was not the factor driving lung functional abnormalities. Lastly, publications have shown that in animal models, males have been highly implicated to have decreased lung function compared to females, but only when an extrinsic factor (such as nicotine or smoke exposure) is involved (135, 168, 241-243). These findings are consistent with asthma development in children, in that the male sex is a risk factor for the development of asthma during childhood (244).

Exaggerated increases in respiratory system resistance in response to inhaled methacholine were previously observed in 7-day old infected BALB/C mice at 109-days of age compared to mock infected controls, consistent with our findings (Fig. 17) (175). One publication involving an IAV infection in 3- or 8-week old BALB/C mice showed a similar finding for airway resistance during acute infection but not at 21 DPI when the infection was cleared (245). Increases in tissue damping, as we found in IAV-infected mice, typically stem from effects in the peripheral lung, such as increased resistance of the 
lung parenchyma or small airways (114). The observed alterations in tissue elastance suggests infection results in loss of the lungs elastic recoil most likely due to parenchymal involvement (246). It is of importance to note that the difference in elastance and tissue elastance arises due to the operational techniques (single frequency and broad band oscillation techniques) used for measuring lung function, and dissects total lung elastance vs parenchymal tissue elastance. Further, differences were greater after methacholine exposure suggesting AHR, which is consistent with what is seen in published literature (175). Lastly at 30-days of age, the end stage for lung development (in mice), alveolarization, is near completion or still on-going (157). Therefore, it is possible that immune cell infiltration and signaling, as well as tissue remodeling due to pH1N1 in the developing parenchymal regions are driving changes resulting in the modest differences in pulmonary function later in life.

In summary, this chapter characterizes a model of $\mathrm{pH} 1 \mathrm{~N} 1$ infection in young mice that provides a tool to study the influence of perinatal events on lung function and allows other investigators a starting point for development of neonatal $\mathrm{pH} 1 \mathrm{~N} 1$ models. We have shown that this model causes lung remodeling at 4 DPI for only the lethal challenge in 14day old mice and for both the lethal and sub-lethal challenges for 30-day old mice. The challenge of virus administered as well as the developmental stage of the lung likely play a role in this finding. Further, 14-day old mice appeared to have more pronounced regional inflammation occurring as a result of infection compared to 30-day old mice. This may be in part due to incomplete development of the $\mathrm{T}_{\mathrm{H}} 2$ cell anti-inflammatory response in the lung although further studies would need to be conducted to confirm this. Lung remodeling is postulated to be mediated, at least in part, by lung fibroblasts due to $\mathrm{pH} 1 \mathrm{~N} 1$ causing 
increased transcripts for tissue remodeling genes early in infection. Importantly, the acute differences at 4 DPI between challenges administered to 30-day old mice groups were reflected in lung functional abnormalities later in life revealed following inhalation of methacholine. Furthermore, the stage of lung development for the 30-day old mice correlates to the stage of lung development for a high school aged human, which was the demographic with the highest incidence for $\mathrm{pH} 1 \mathrm{~N} 1$ infection in 2009. Therefore, this data perhaps provides rationale for monitoring the lung function of individuals who were infected as adolescents with the 2009 Swine Flu. 
CHAPTER IV: NICOTINE EXPOSURE ACCOMPANIED WITH EARLY LIFE

PANDEMIC H1N1 INFECTION LEADS TO MURINE ADULT LUNG

DYSFUNCTION 


\section{$\underline{\text { Background }}$}

Smoking and/or exposure to ETS leads to the development of a plethora of diseases including but not limited to diseases in the lung such as COPD and asthma (247). These pulmonary diseases affect millions of people worldwide (causing a substantial monetary burden), and are characterized by chronic airways inflammation, tissue remodeling, and airway hyperreactivity $(1,3,4)$.

Our laboratory and several other investigators have proposed that the development of OAD is greatly influenced (and perhaps pre-determined) by exposure to extrinsic factors (such as ETS or nicotine and respiratory tract infections) during the period of lung development $(42,47,64,68,72,124,126,130-132,174,175,178)$. ETS is considered a major risk factor for the development of $\mathrm{OAD}$, and maternal smoking during pregnancy (fetal nicotine exposure) has been independently associated with an increased risk of asthma and subsequently lung function abnormalities in adulthood (42, 47, 64, 124, 126). Determining which components of ETS that play a role in disease development at this point of time is difficult, due to the vast number of components and the variation of components due to combustion reactions occurring during tobacco smoking. Our previous data (Chapter II) address the effect of nicotine, which is capable of crossing the placenta to the fetus and mimics concentration levels (along with its metabolite cotinine) in the mother, on lung function both short (8-weeks of age) and long term (210-days of age) in mice (42, 57, 61, 248). We found that perinatal nicotine exposure affected lung branching morphogenesis and were associated with alterations in lung function; these changes appeared to be mediated via a 7 nAChRs $(42,64,196)$. More recently, we showed that extension of perinatal nicotine exposure into adulthood resulted in increased peri-airway 
collagen deposition and increased alveolar size. These changes were associated with alterations in lung function, thereby supporting our hypothesis that early life exposures enhance susceptibility to chronic lung disease later in life.

Similarly, lower respiratory viral infections, such as IAV, during the alveolar stage of lung development, have also been implicated in the development of OADs (Chapter III) $(46,67-70,175)$. Interestingly, nicotine has been shown to increase the viral titer for IAV, H1N1 in the lungs of mice (249). Analyzed epidemiological data found that the risk factors associated with the development of OADs later in life due to a respiratory tract infection are susceptibility to infection (i.e. age and/or immunocompromised state) and the intensity of the response of the host to the infectious agent (bacteria or viral) rather than the agent itself (174). Therefore, we would expect similar findings for other infections (such as RSV or possibly rhinoviruses) occurring during the alveolarization stage of lung development (with or without nicotine). Furthermore, IAV is known to be associated with lung functional abnormalities later in life when infection happens early in life (both humans and mice) (Chapter III), and causes pulmonary exacerbations in persons with COPD $(68,72$, 174, 175, 178). Lastly, correlative to epidemiological data, our previous data (Chapter III) along with others show that murine infections with IAV have been published to lead to lung functional abnormalities with age (210-days of age), increasing airway resistance, tissue damping, and tissue elastance $(175,224,250)$.

Together, these published and unpublished findings strongly suggest that perinatal nicotine exposure and early life respiratory tract infection (H1N1) affects the mammalian lung in ways that may potentiate the future development of OADs. These results have lead us to question whether, nicotine exposure along with early postnatal IAV pH1N1 infection 
act in concert to promote abnormalities in airway structure and function that persist through adulthood. Herein, we report observations and suggest that this is indeed the case.

\section{Materials and Methods}

All materials and methods are identical to the above chapters unless noted below. The experimental outline followed is shown in Figure 18, and combines the designs from the previous chapters.

\section{Murine Lung Function Testing}

Untreated (mock infected) mice $(\mathrm{n}=19$; male $=10$, female $=9)$, mice in the transient nicotine exposure group $(\mathrm{n}=18$; male $=10$, female $=8)$, mice in the chronic nicotine exposure group $(\mathrm{n}=20$; male $=10$, female $=10)$, mice in the pH1N1 infected $\left(1 \times 10^{1.7}\right.$ ma-CA/07/09 viruses) group $(n=19 ;$ male $=9$, female $=10)$, mice in the transient nicotine exposed plus $\mathrm{pH} 1 \mathrm{~N} 1$ infected $\left(1 \times 10^{1.7} \mathrm{ma}-\mathrm{CA} / 07 / 09\right.$ viruses $)$ group $(\mathrm{n}=20$; male $=10$, female $=10)$, and mice in the chronic nicotine exposed plus pH1N1 infected $\left(1 \times 10^{1.7}\right.$ ma-CA/07/09 viruses) group $(\mathrm{n}=21$; male $=10$, female $=11)$ were subjected to PFT. Pulmonary function at baseline and after methacholine challenge $(0-50 \mathrm{mg} / \mathrm{mL}$, inhaled) was measured by forced oscillation using the flexiVent system (SCIREQ, Montreal, Quebec, Canada) as previously described $(137,138)$. 


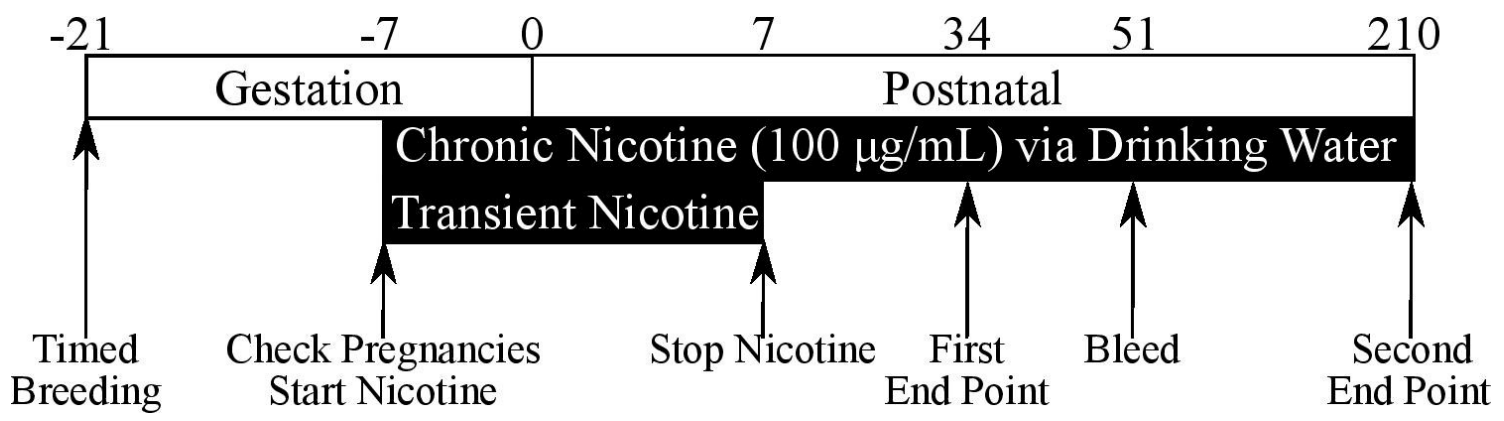

\section{Figure 18. Perinatal Nicotine Exposure with pH1N1 Infection Experimental Design}

Timed pregnancies were produced by pairing mice for 48 hours and then assessing for conception after 14 days, at which point nicotine was administered via the drinking water at $(100 \mu \mathrm{g} / \mathrm{mL})$ for pregnant mothers. Nicotine exposure lasted until postnatal day 7 or until the completion of the experiment (postnatal day 210), when pulmonary function tests were conducted. Infection with pH1N1 occurred at 30-days of age and animals were bleed at 51-days of age (21 DPI). Endpoints were implemented at 34 and 210-days of age. 


\section{Statistics}

SAS statistical software was used to analyze lung function data. A correlation chart for pairwise comparison between all six outcome parameters (resistance, compliance, elastance, Newtonian resistance, tissue damping, and tissue elastance) was generated. For both chronic nicotine with $\mathrm{pH} 1 \mathrm{~N} 1$ and transient nicotine with $\mathrm{pH} 1 \mathrm{~N} 1$ infection outcome parameters showed strong correlation for all parameters to each other except for Newtonian resistance and compliance (Fig. 19\&20). A 3-factor ANOVA was then implemented with the factors representing experimental group, sex type, and dose of methacholine used. Descriptive statistics were calculated for the entire study population as well as within each factor. Rigorous analysis involved the main effects for 3-factor ANOVA along with 2-way interactions (group*sex, group*dose, sex*dose). Due to the outcomes of interaction of the outcome parameters and the previous interaction of the exposures alone, we conducted a 3-factor ANOVA as before. Rather than subset analysis, we have used contrast to test the main effects (such as untreated-mock infected versus pH1N1; untreated-mock infected versus transient or chronic nicotine, and untreated-mock infected versus transient or chronic nicotine plus $\mathrm{pH} 1 \mathrm{~N} 1, \mathrm{pH} 1 \mathrm{~N} 1$ versus transient or chronic nicotine, $\mathrm{pH} 1 \mathrm{~N} 1$ versus transient or chronic nicotine plus $\mathrm{pH} 1 \mathrm{~N} 1$, and transient or chronic nicotine versus transient or chronic nicotine plus $\mathrm{pH} 1 \mathrm{~N} 1)$, which increases power to detect significant effects (143, 144). Resistance, compliance, elastance, Newtonian resistance, tissue damping, and tissue elastance were examined at baseline and after stimulation with increasing concentrations of aerosolized methacholine from $0-50 \mathrm{mg} / \mathrm{mL}$. For statistical analysis, we only evaluated responses at baseline and after 25 and $50 \mathrm{mg} / \mathrm{mL}$ of methacholine. Using GraphPad Prism 
7.03 the following statistical test were implemented as previously described in previous chapters. 


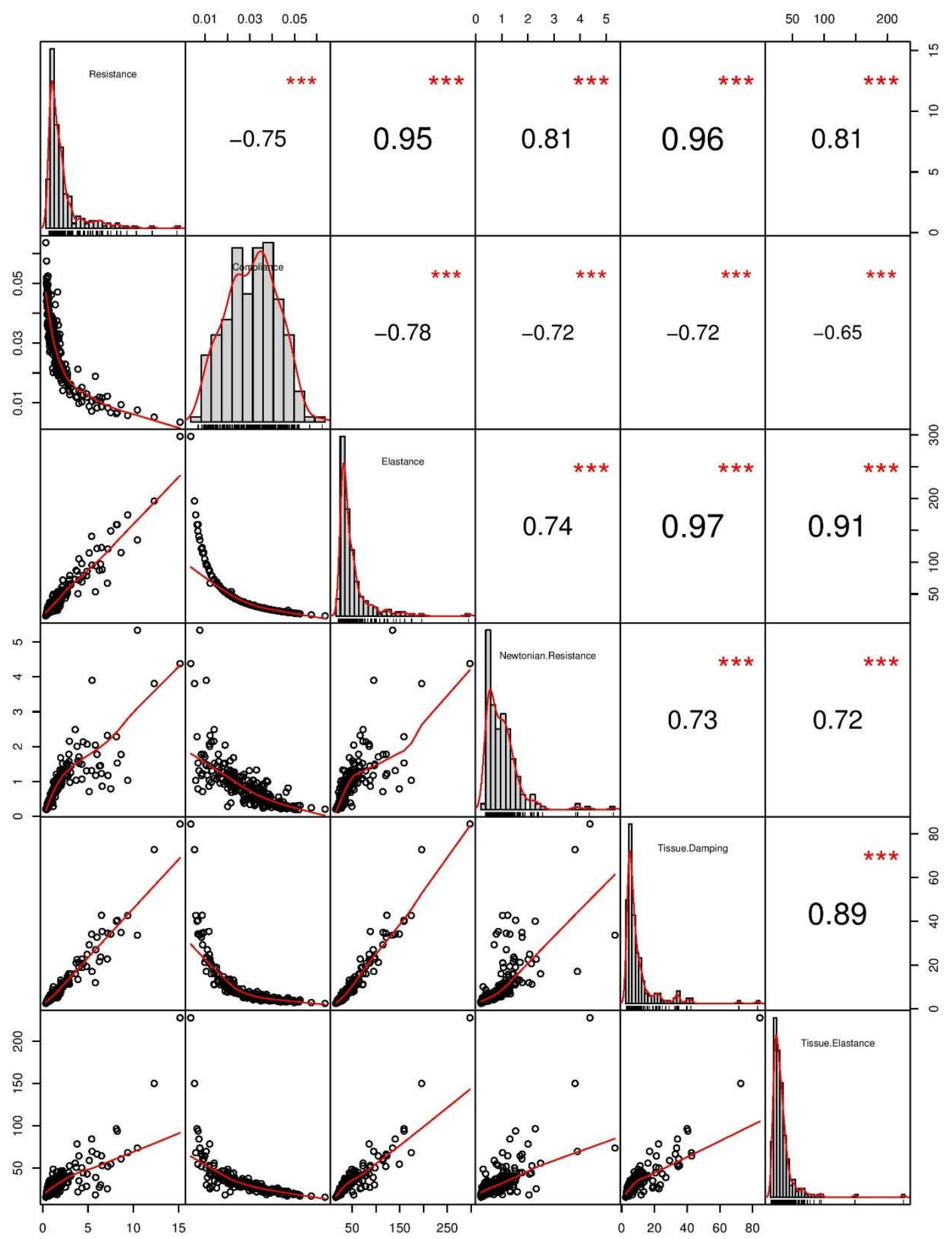

Figure 19. Correlation of Pulmonary Function Test Outcome Parameters for

\section{Transient Nicotine with pH1N1 Statistical Analysis (All Groups)}

All data used for analysis in SAS 3 Factor ANOVA. Graphical representation of correlation represented in bottom left of figure. Fit of data to linear line of regression represented in top right of figure. 


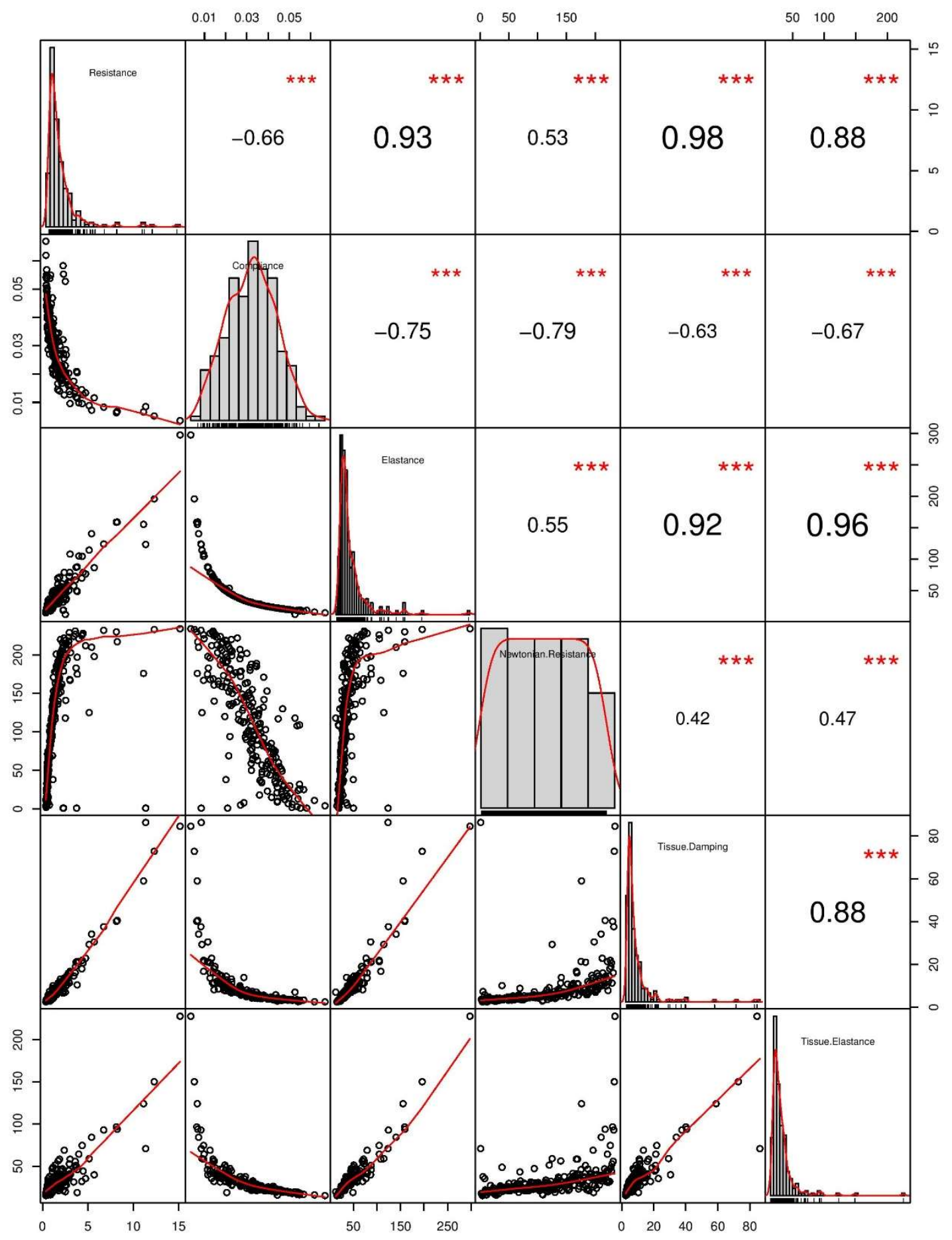

Figure 20. Correlation of Pulmonary Function Test Outcome Parameters for

\section{Chronic Nicotine with pH1N1 Statistical Analysis (All Groups)}

All data used for analysis in SAS 3 Factor ANOVA. Graphical representation of correlation represented in bottom left of figure. Fit of data to linear line of regression represented in top right of figure. 


\section{$\underline{\text { Results }}$}

\section{Growth Stunting After Infection with $\mathrm{pHINI}$}

Nicotine exposure did not affect weight gain (data not shown). However, significant differences in percent body weight (from time of infection) were found for all infected experimental groups compared to untreated mice, with statistics only being assessed versus mice without either factor (i.e. untreated or also referred to as mock infected). When infection with $\mathrm{pH} 1 \mathrm{~N} 1$ alone occurred, significant differences in weight began at 6 days post infection (DPI) (compared to untreated) (Fig. 21). Interestingly nicotine exposures with $\mathrm{pH} 1 \mathrm{~N} 1$ infection caused significant differences in percent weight loss from time of infection quicker than in mice infected with $\mathrm{pH} 1 \mathrm{~N} 1$ alone (starting at $6 \mathrm{DPI}$ ), with chronically exposed mice being different at 5 DPI and transient exposed mice being different at 3 DPI. Significant differences between all groups remained until when weighing mice ceased (with exception of 15 DPI for pH1N1 alone versus untreated). Furthermore, female mice showed more pronounced differences from uninfected controls.

Interestingly, transient nicotine with $\mathrm{pH} 1 \mathrm{~N} 1$ infection was found to elicit a more severe effect on percent weight loss from time of infection compared to both $\mathrm{pH} 1 \mathrm{~N} 1$ alone and with chronic nicotine treatment. It was found that transient nicotine exposure with pH1N1 was different from the other two pH1N1-infected groups beginning at 8 DPI and proceeding until 16 DPI (chronic with pH1N1) and 17 DPI (pH1N1 alone, statistical difference was not found at 9 DPI) (Fig. 21). No significant differences were discovered between $\mathrm{pH} 1 \mathrm{~N} 1$ alone chronic nicotine exposure with $\mathrm{pH} 1 \mathrm{~N} 1$ at any time. Although, differences were discovered between infected groups acutely all groups were statistically not different at the time of infection or after 210-days of age (Fig. 21). 

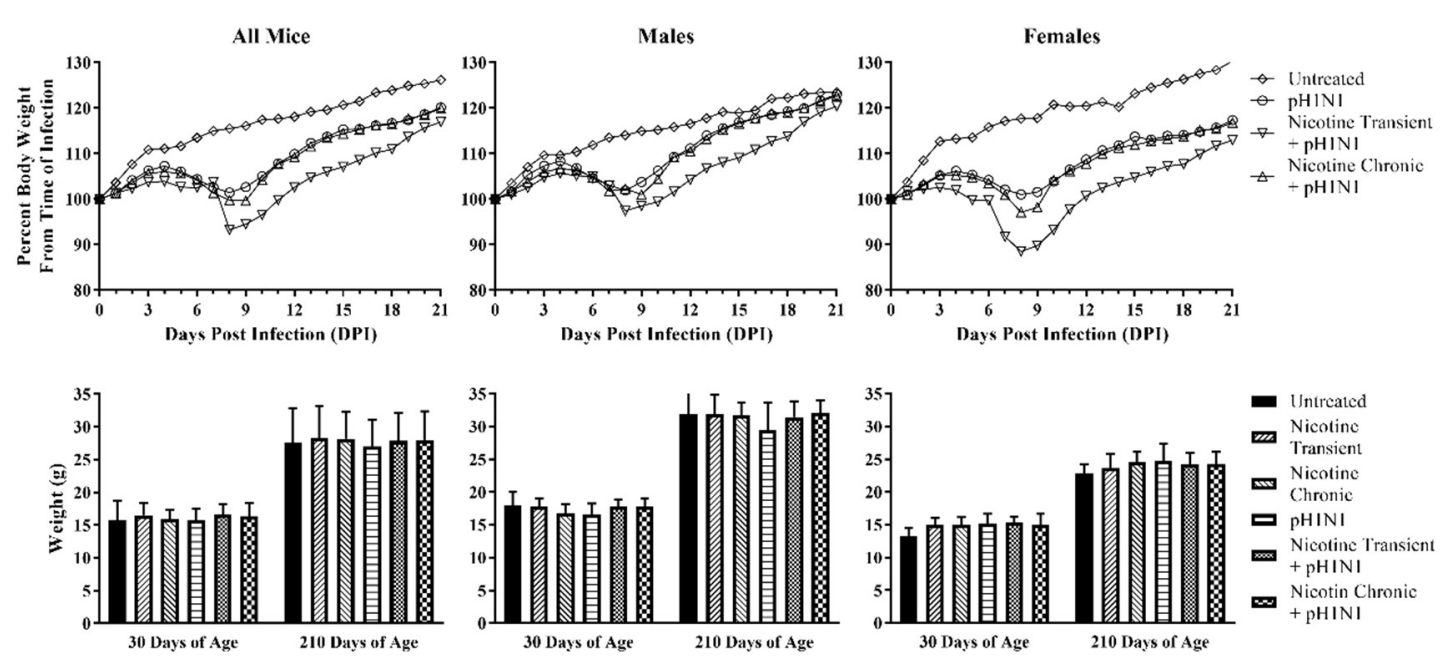

Figure 21. Weights of Perinatal Nicotine Exposed Mice with pH1N1 Infection

\section{Assessed for Lung Functional Abnormalities}

Average percent body weights (30 until 51-days of age) and average weights (30 and 210-days of age) of mice groups for the indicated exposures. Data was imported into GraphPad Prism 7.03, the percent from time of infection weights were calculated along with their standard deviations then compared using two-way ANOVA analysis with a Turkey post-test to correct for multiple comparisons. The weights at 30 and 210days of age were compared using unpaired t-test analysis with a Holm-Sidak multiple comparison test. A linear regression analysis was also conducted between groups in GraphPad, with no differences reported. 


\section{Chronic Nicotine with or without pH1N1 Pulmonary Function Abnormalities}

The mice represented in Figure 21 were allowed to mature until 210-days of age at which point their lung function was assessed. Chronic nicotine exposure alone caused differences (compared to the untreated-mock infected mice group) in lung compliance ( $p$ $<0.001)$ and Newtonian resistance $(\mathrm{p}=0.049)($ Fig. 22). Infection with $\mathrm{pH} 1 \mathrm{~N} 1$ alone, was found to cause drastic alterations in lung function, compared to untreated (which were mock infected) animals, affecting all outcome parameters with the exception of lung compliance (resistance $p=0.002$, elastance $p=0.001$, Newtonian resistance $p=0.008$, tissue damping $p=0.002$, and tissue elastance $p=0.002$ ) (Fig. 22). Interestingly the combination of chronic nicotine exposure with $\mathrm{pH} 1 \mathrm{~N} 1$ infection was found to only cause changes in resistance $(p=0.005)$ and tissue damping $(p=0.015)$ (summarized in Table 10). In addition, differences in outcome parameters were found to be statistically different (with males being more severely affected) for resistance $(p=0.027)$, Newtonian resistance $(\mathrm{p}=0.035)$, and tissue damping (all are airway associated parameters) $(\mathrm{p}=0.035)$, but not for compliance, elastance, and tissue elastance (tissue associated parameters) (Fig. 22). All other group comparison p-values can be seen in the in Figure 22. 

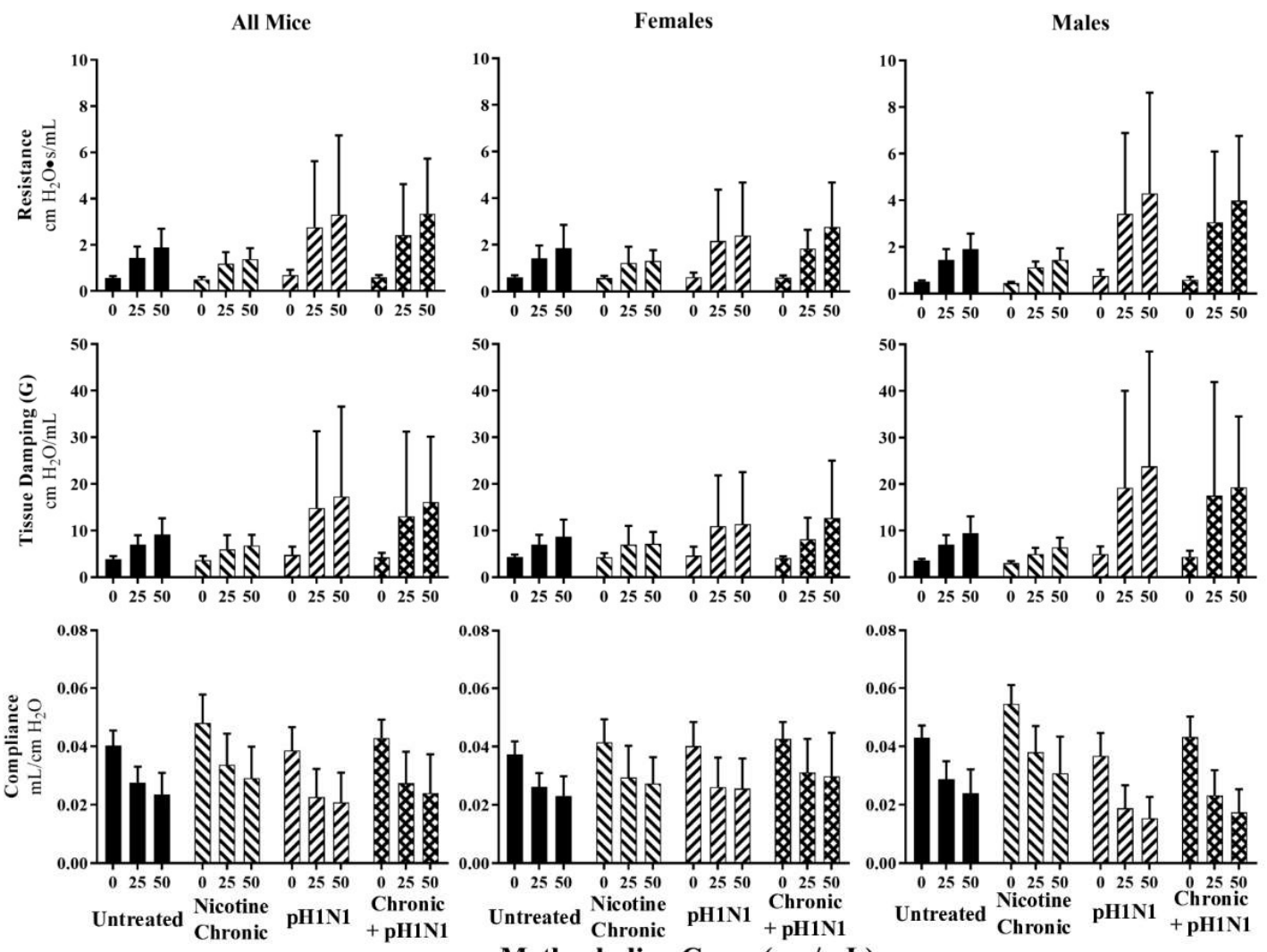

Methacholine Conc. ( $\mathrm{mg} / \mathrm{mL})$

\begin{tabular}{|l|c|c|c|c|c|c|}
\hline \multicolumn{1}{|c|}{ Comparison } & Resistance & Compliance & Elastance & Newtonian Resistance & Tissue Damping & Tissue Elastance \\
\hline Untreated vs. Nicotine Chronic & 0.393 & $<0.001 \uparrow$ & 0.377 & $0.049 \downarrow$ & 0.535 & 0.380 \\
\hline Untreated vs. pHIN1 & $0.002 \uparrow$ & 0.070 & $0.001 \uparrow$ & $0.008 \uparrow$ & $0.002 \uparrow$ & $0.002 \uparrow$ \\
\hline Untreated vs. Chronic + pHIN1 & $0.005 \uparrow$ & 0.576 & 0.292 & 0.181 & $0.015 \uparrow$ & 0.454 \\
\hline Chronic vs. pHIN1 & $<0.001 \uparrow$ & $<0.001 \downarrow$ & $<0.001 \uparrow$ & $<0.001 \uparrow$ & $<0.001 \uparrow$ & $<0.001 \uparrow$ \\
\hline Chronic vs. Chronic + pHIN1 & $<0.001 \uparrow$ & $0.001 \downarrow$ & $0.049 \uparrow$ & $0.001 \uparrow$ & $0.002 \uparrow$ & 0.100 \\
\hline pHIN1 vs. Chronic +pHIN1 & 0.666 & $0.016 \uparrow$ & $0.019 \downarrow$ & 0.178 & 0.482 & $0.015 \downarrow$ \\
\hline Females vs. Males & $0.027 \uparrow$ & 0.640 & 0.069 & $0.035 \uparrow$ & $0.035 \uparrow$ & 0.427 \\
\hline $\mathbf{0}$ vs. 25 & $<0.001 \uparrow$ & $<0.001$ & $<0.001 \uparrow$ & $<0.001 \uparrow$ & $<0.001 \uparrow$ & $<0.001 \uparrow$ \\
\hline $\mathbf{0}$ vs. 50 & $<0.001 \uparrow$ & $<0.001$ & $<0.001 \uparrow$ & $<0.001 \uparrow$ & $<0.001 \uparrow$ & $<0.001 \uparrow$ \\
\hline $\mathbf{2 5}$ vs. 50 & $0.040 \uparrow$ & 0.018 & $0.024 \uparrow$ & $0.004 \uparrow$ & $0.195 \uparrow$ & $0.078 \uparrow$ \\
\hline
\end{tabular}

Figure 22. Pulmonary Function Test Results for Chronic Nicotine Exposure with

\section{pH1N1 Infection at 210-Days of Age}

Lung function was assessed C57BL/6 at 210-days of age by forced oscillation technique using a SCIREQ flexiVent system. Untreated (mock infected) mice $(n=19$; male $=10$, female $=9$ ), mice in the chronic nicotine exposure group $(n=20 ;$ male $=10$, female $=10)$, mice in the pH1N1 exposure group $(n=19$; male $=9$, female $=10)$, and mice in the chronic plus pH1N1 exposure group $(\mathrm{n}=21 ;$ male $=10$, female $=11)$ were tested. Lung function was measured at baseline and after challenge with increasing concentrations of aerosolized methacholine. Averages of results (only including values where the COD was $\geq 95 \%$ ) were taken 
for each individual mouse. SAS statistical software was used to conduct 3-factor ANOVA without interaction terms. Results were declared significant at 5\% significance level $(\mathrm{p}<0.05)$. Error bars represent standard deviation from the mean. P-values are represented in the table with significant results having an arrow dictating the direction of the second group in each row compared to the first group. 
Table 10. Summary of Chronic Nicotine with pH1N1 Pulmonary Function Testing

\begin{tabular}{|c|c|c|c|c|c|c|}
\hline \multicolumn{2}{|c}{ Resistance } & Compliance & Elastance & $\begin{array}{c}\text { Newtonian } \\
\text { Resistance }\end{array}$ & $\begin{array}{c}\text { Tissue } \\
\text { Damping }\end{array}$ & $\begin{array}{c}\text { Tissue } \\
\text { Elastance }\end{array}$ \\
\hline $\begin{array}{c}\text { Chronic } \\
+\mathrm{pH} 1 \mathrm{~N} 1\end{array}$ & $\uparrow$ & No Change & $\begin{array}{c}\text { No } \\
\text { Change }\end{array}$ & No Change & $\uparrow$ & $\begin{array}{c}\text { No } \\
\text { Change }\end{array}$ \\
\hline
\end{tabular}

Arrows indicate direction of parameter compared to untreated-mock infected control

Blue indicates alteration in function driven by males

Black indicates alteration in function not driven by either sex 


\section{Chronic Nicotine with pH1N1 Related Lung Structural Abnormalities}

Lungs from mice assessed for lung function above were later examined using histological analysis. Using $\mathrm{H} \& \mathrm{E}$ stained slides mean linear intercept quantitation found that only chronic nicotine alone caused an increase in alveolar volume compared to all other treatment groups (Fig. 23 A\&C). Differences discovered in alveolar size were found to be independent of sex type. Using Masson's Trichrome staining, collagen deposition was quantitated. This revealed that both chronic nicotine exposure and $\mathrm{pH} 1 \mathrm{~N} 1$ infection, alone but not together, were different from untreated mice (with exception of males for pH1N1 alone) (Fig. 23 B\&D). For pH1N1 alone we estimated that $\sim 30-50 \%$ of the large central conducting airways had collagen deposition occurring around them. Chronic nicotine exposure followed by $\mathrm{pH} 1 \mathrm{~N} 1$ infection was significantly different from chronic nicotine alone, but comparing to untreated mice, this difference was only detected in females. In addition, for female mice, all exposure groups had relatively the same collagen staining occurring (with exception to untreated-mock infected). Chronic nicotine exposure resulted in the most collagen deposition, followed in intensity by $\mathrm{pH} 1 \mathrm{~N} 1$, followed by the combination in all mice and male mice. Regarding collagen deposition, pH1N1 and chronic nicotine exposure (separately) affected about the same percentage of large airways $(\sim 30-50 \%)$, whereas the combination of chronic nicotine with $\mathrm{pH} 1 \mathrm{~N} 1$ affected collagen deposition slightly more than uninfected-mock controls $(\sim 20 \%)$. Furthermore, collagen deposition was found to be primarily occurring around the large airways in the lung and not in the more peripheral parenchymal regions. 

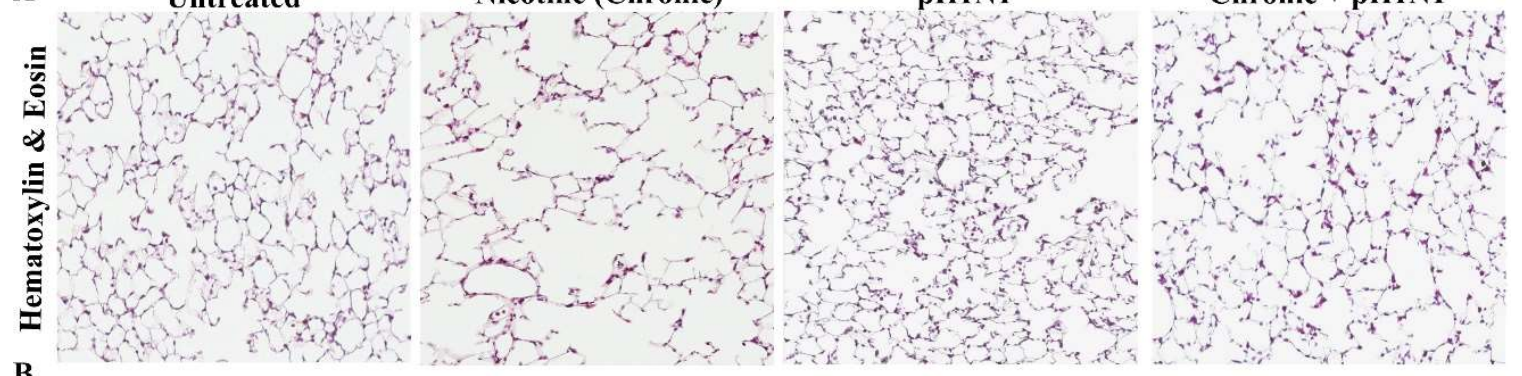

B
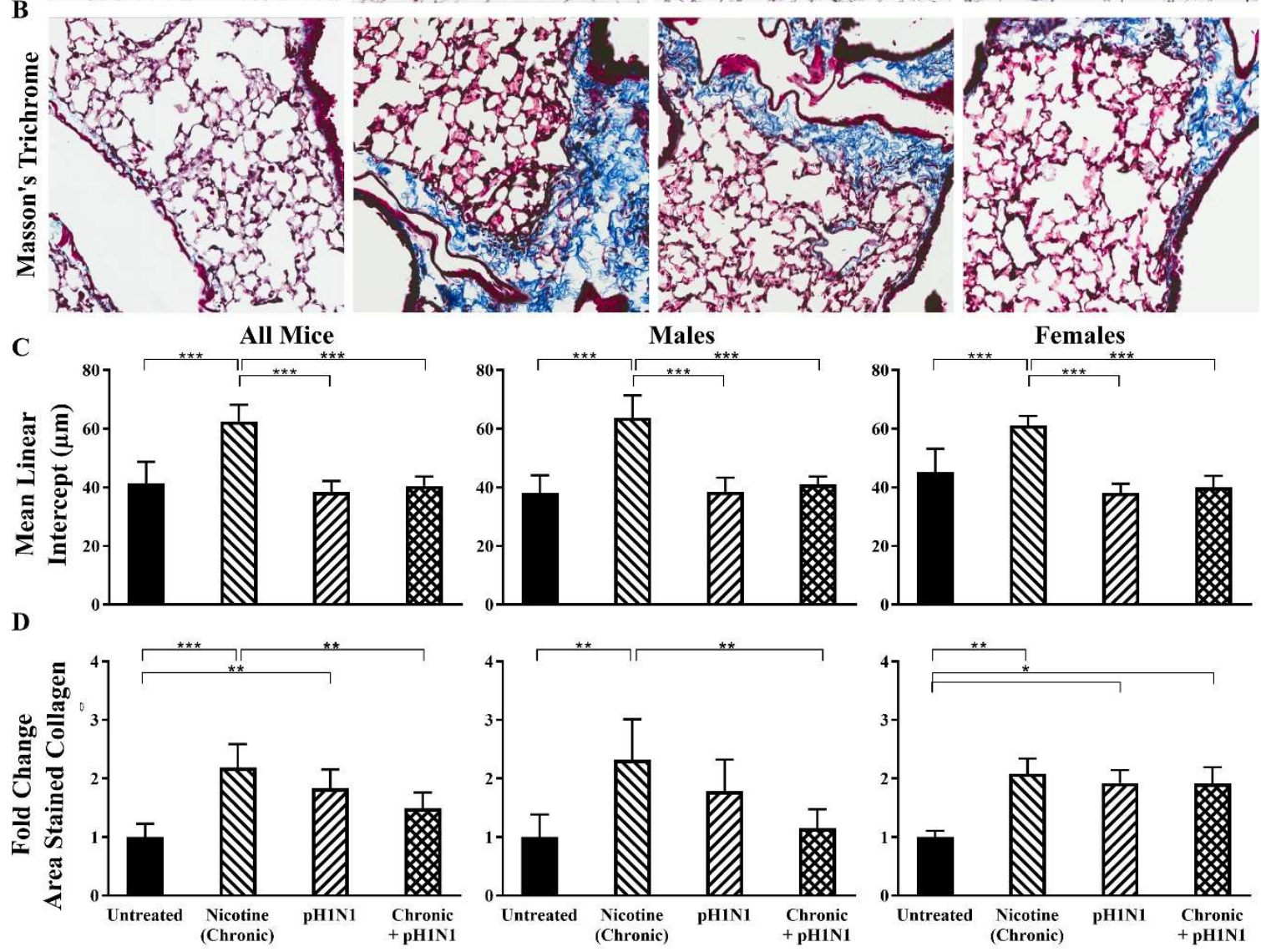

Figure 23. Lung Histology of Chronic Nicotine Exposure and pH1N1 Infection

\section{(Alone or in Combination) at 210-Days of Age}

Lung histology in C57BL/6 mice, including H\&E staining at 20x magnification (A) and Mason's Trichrome staining at 20x magnification (B). Alveolar mean linear intercept quantification $(\mathbf{C})$ and the percent of total lung area stained for collagen (D). In GraphPad Prism 7.03 one-way ANOVA analyses were conducted with all groups using a Bonferroni test to correct for multiple comparisons. Multiplicity adjusted p-values were reported for each comparison. Error bars correspond the standard deviation (C) or error (D) the mean. Differences are represented vs. mock infected controls, $\mathrm{p}<0.05:^{*}, \mathrm{p}<0.01:^{* *}, \mathrm{p}<0.001$ : $^{* * *}$. 
Transient Nicotine Exposure with pH1N1 Infection-Related Pulmonary Abnormalities

Unlike the chronic nicotine exposed groups, transient nicotine caused no significant differences in pulmonary function compared to the untreated group (210-days of age) (Fig. 24). Similar to the above, this separate analysis resulted in identical findings for the $\mathrm{pH} 1 \mathrm{~N} 1$ infected group compared to the untreated-mock infected group in the previous analysis involving chronic nicotine exposure. Surprisingly and unlike the above, the combination of nicotine (transient) with pH1N1 drove drastic, significant changes in lung function (resistance $\mathrm{p}=0.022$, compliance $\mathrm{p}=0.002$, elastance $\mathrm{p}=0.007$, Newtonian resistance $\mathrm{p}$ $=0.014$, tissue damping $\mathrm{p}=0.006$, and tissue elastance $\mathrm{p}=0.001$ ), compared to untreated controls (Fig. 24) (summarized in Table 11). Transient nicotine exposure with pH1N1 caused differences in lung compliance (increased at baseline), which was not found when either factor was acting alone $(\mathrm{p}=0.002)$. The combination also showed a trend to have a synergistic effect on increasing airway resistance and Newtonian resistance (data not shown) (Fig. 24). Similar to the above, differences discovered for resistance, elastance Newtonian resistance, and tissue damping were associated to sex type (with males being more severely affected), but not for compliance and tissue elastance (Fig. 24). 


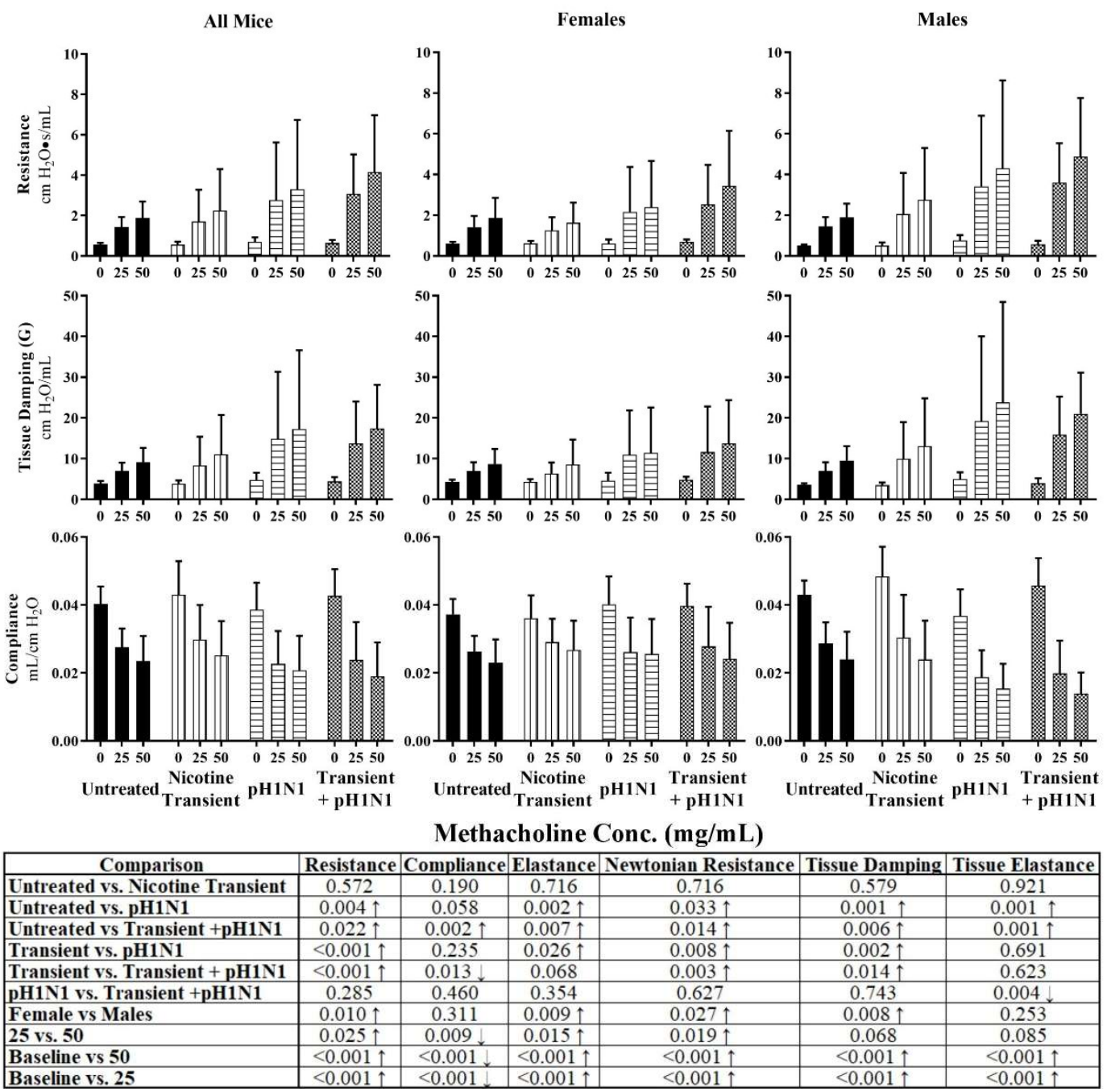

Figure 24. Pulmonary Function Test Results for Transient Nicotine Exposure with

\section{pH1N1 Infection at 210-Days of Age}

Lung function was assessed C57BL/6 at 210-days of age by forced oscillation technique using a SCIREQ flexiVent system. Untreated (mock infected) mice $(n=19$; male $=10$, female $=9)$, mice in the transient nicotine exposure group $(\mathrm{n}=18$; male $=10$, female $=8)$, mice in the $\mathrm{pH} 1 \mathrm{~N} 1$ exposure group $(\mathrm{n}=19$; male $=$ 9 , female $=10)$, and mice in the transient plus pH1N1 exposure group $(\mathrm{n}=20 ;$ male $=10$, female $=10)$ were tested. Lung function was measured at baseline and after challenge with increasing concentrations of aerosolized methacholine. Averages of results (only including values where the COD was $\geq 95 \%$ ) were taken for each individual mouse. SAS statistical software was used to conduct 3-factor ANOVA without interaction terms. Results were declared significant at 5\% significance level $(\mathrm{p}<0.05)$. Error bars represent 
standard deviation from the mean. P-values are represented in the table with significant results having an arrow dictating the direction of the second group in each row compared to the first group. 
Table 11. Summary of Transient Nicotine with pH1N1 Pulmonary Function Testing

\begin{tabular}{|c|c|c|c|c|c|c|}
\hline & Resistance & Compliance & Elastance & $\begin{array}{l}\text { Newtonian } \\
\text { Resistance }\end{array}$ & $\begin{array}{c}\text { Tissue } \\
\text { Damping }\end{array}$ & $\begin{array}{c}\text { Tissue } \\
\text { Elastance }\end{array}$ \\
\hline $\begin{array}{l}\text { Transient } \\
+\mathrm{pH} 1 \mathrm{~N} 1\end{array}$ & 个 & $\uparrow_{\mathrm{B}}$ & $\mathrm{B}$ & 个 & 个 & 个 \\
\hline \multicolumn{7}{|c|}{$\begin{array}{l}\text { Arrows indicate direction of parameter compared to untreated-mock infected control } \\
\text { Blue indicates alteration in function driven by males } \\
\text { Black indicates alteration in function not driven by either sex } \\
\text { "B" indicates baseline }\end{array}$} \\
\hline
\end{tabular}


Transient Nicotine with pH1N1 Related Lung Structural Abnormalities

Lungs from the mice previously assessed for pulmonary function were also examined using histological analysis. The alveolar volume for transient nicotine with pH1N1 was found to be increased and statistically different from the untreated (mock infected) group (with expectation to females) as well as both factors alone (for both males and females) (Fig. 25 A\&C). The distance between alveolar septum increased $\sim 50 \%$ in transient nicotine with pH1N1 compared to untreated-mock infected controls. Alveolar septum distances were uniform in each of the groups at 210-days of age. At 34-days of age, the alterations detected were less uniform, suggesting the process was ongoing.

As described before, for collagen deposition, $\mathrm{pH} 1 \mathrm{~N} 1$ alone was statistically different from the untreated group and was also found to be different from the transient nicotine group with or without pH1N1 (all mice and females) (Fig. 23\&25). Due to only seeing differences between untreated mice versus transient nicotine with $\mathrm{pH} 1 \mathrm{~N} 1$ in mean linear intercept, we quantitated the alveolar size earlier in life (34-days of age, 4 DPI). It was discovered that an increase in alveolar size was occurring at 34-days of age compared to untreated controls, the distance between alveolar septum was increased $\sim 20 \%$ for transient nicotine with $\mathrm{pH} 1 \mathrm{~N} 1$ (Fig. 26). In addition, $\mathrm{pH} 1 \mathrm{~N} 1$ infection following transient nicotine exposure resulted in less inflammation than discovered with pH1N1 alone, which was primarily located around large airways but also was found parenchymal regions although not as pronounced. Furthermore, assessing the two-time points for alveolar size it was found that the mean linear intercept was greater at 210-days of age compared to 34days of age for transient nicotine with $\mathrm{pH} 1 \mathrm{~N} 1$, but not for untreated-mock infected mice (Fig. 27). 


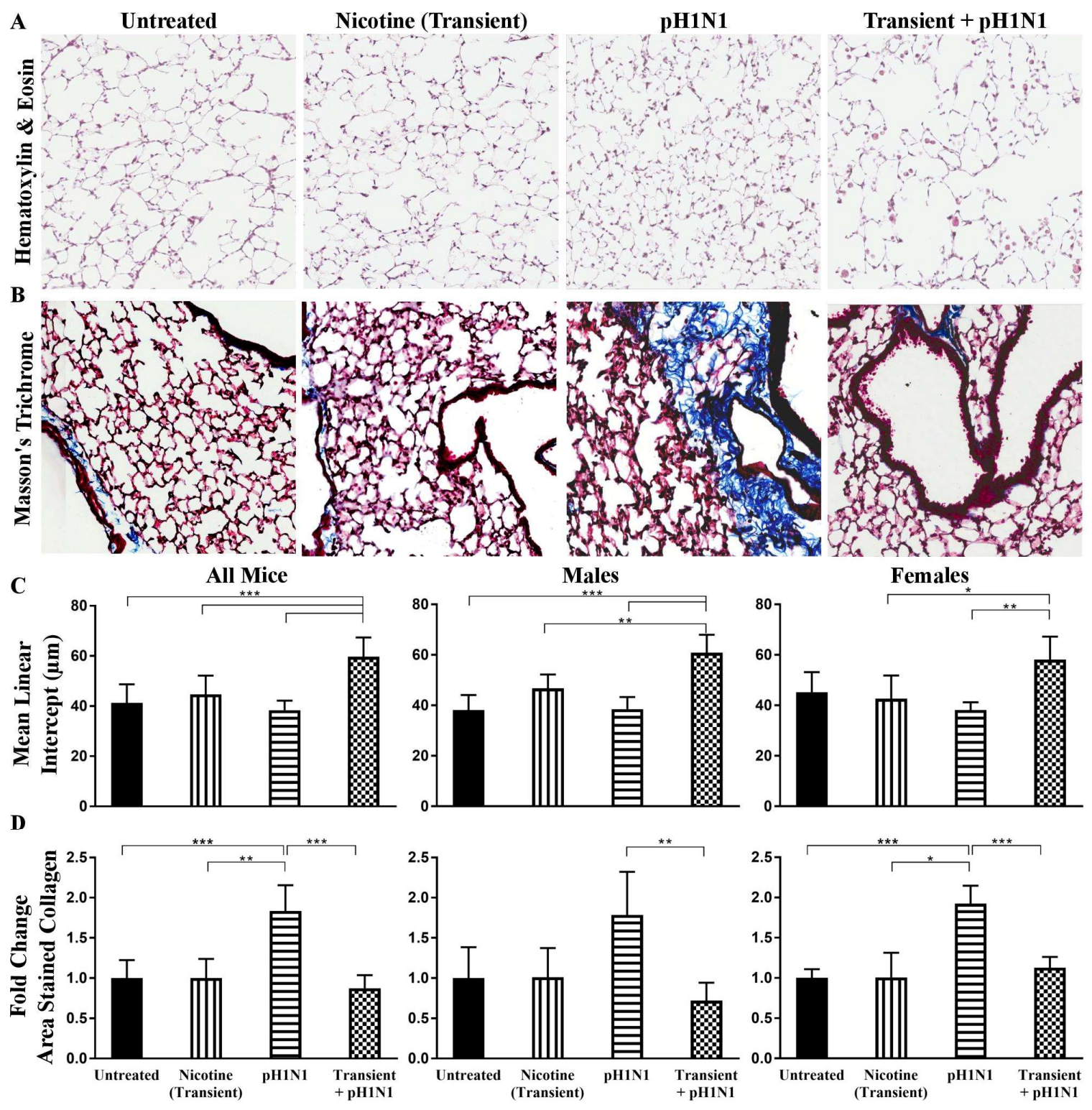

Figure 25. Lung Histology of Transient Nicotine Exposure and pH1N1 Infection

\section{(Alone or in Combination) at 210-Days of Age}

Lung histology in C57BL/6 mice, including H\&E staining at 20x magnification (A) and Mason's Trichrome staining at 10x magnification (B). Alveolar mean linear intercept quantification $(\mathbf{C})$ and the percent of total lung area stained for collagen (D). In GraphPad Prism 7.03 one-way ANOVA analyses were conducted with all groups using a Bonferroni test to correct for multiple comparisons. Multiplicity adjusted p-values were reported for each comparison. Error bars correspond the standard deviation $(\mathbf{C})$ or error $(\mathbf{D})$ the mean. Differences are represented vs. mock infected controls, $\mathrm{p}<0.05:^{*}, \mathrm{p}<0.01:^{* *}, \mathrm{p}<0.001$ : $^{* * *}$. 

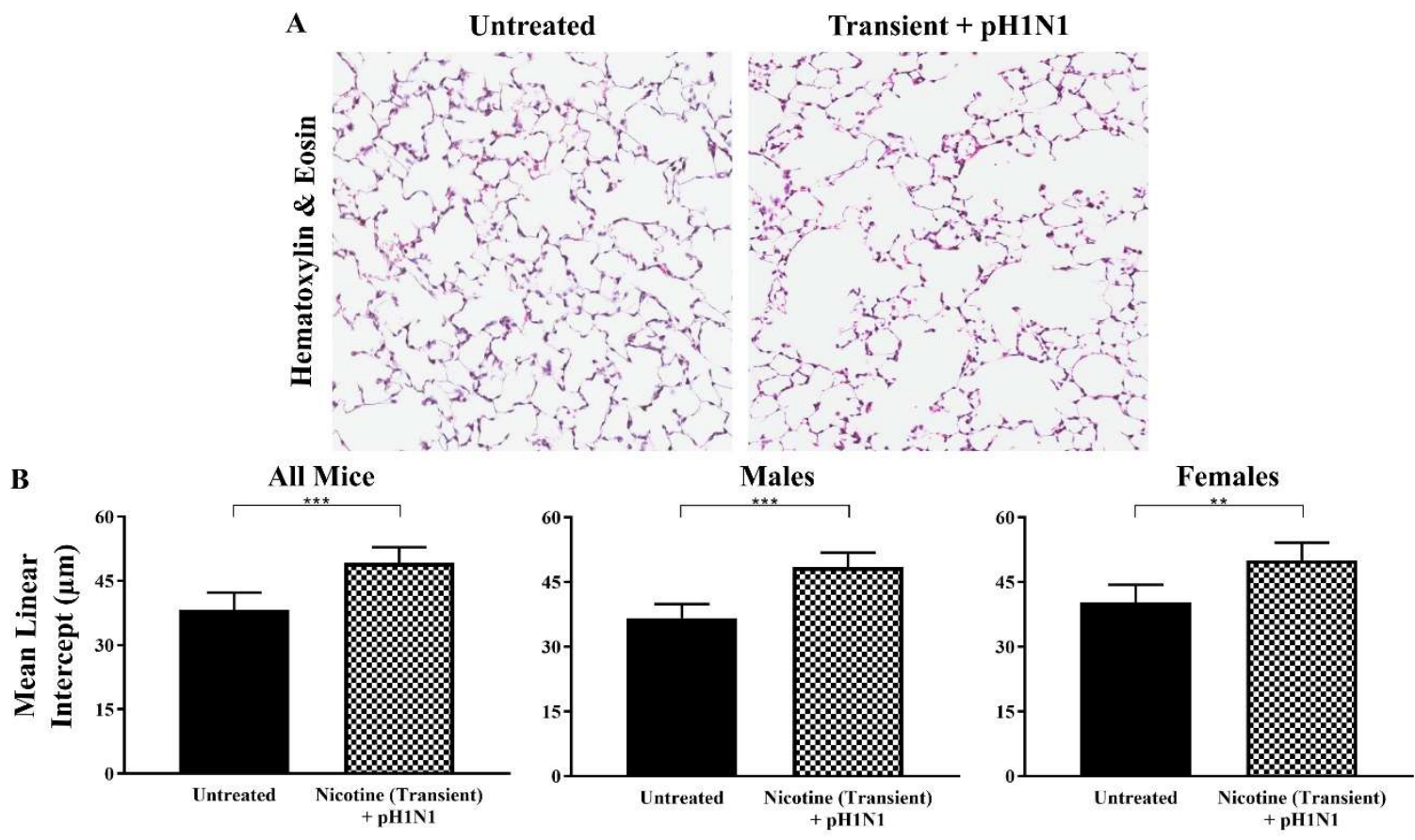

Figure 26. Lung Histology of Transient Nicotine Exposure with pH1N1 Infection at

\section{4-Days of Age}

Lung histology in C57BL/6 mice, including H\&E staining at 20x magnification (A) and corresponding alveolar mean linear intercept quantification (B). In GraphPad Prism 7.03 two-tailed t-tests compared to untreated controlled were implemented. Multiplicity adjusted p-values were reported for each comparison. Error bars correspond the standard deviation of the mean. Differences are represented as, $\mathrm{p}<0.01$ : ${ }^{* *}, \mathrm{p}<$ $0.001: * * *$. 
A

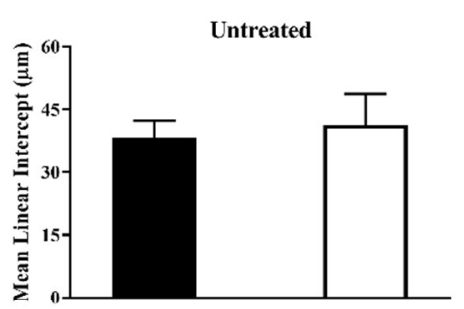

B

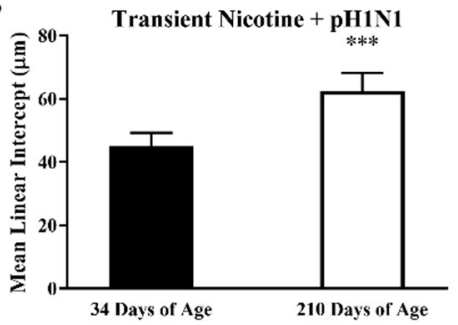

Males

Untreated

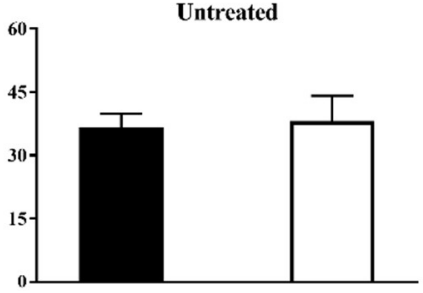

Transient Nicotine + pH1N1

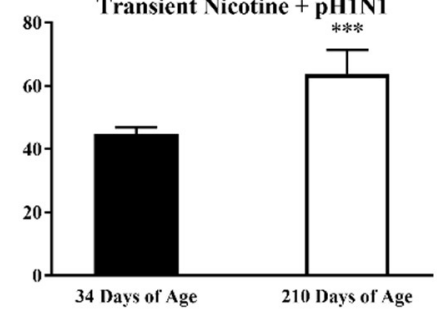

Females

Untreated

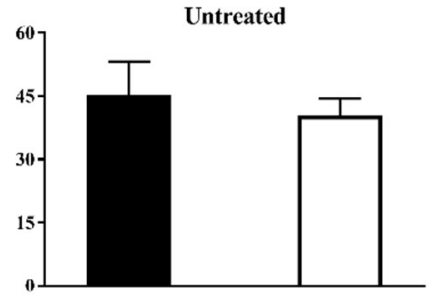

Transient Nicotine $+\underset{* * *}{\mathbf{p H} 1 \mathrm{~N} 1}$

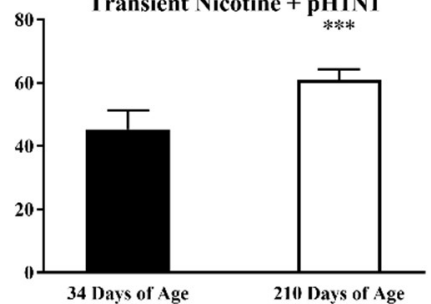

Figure 27. Alveolar Septum Quantification Over Time in Transient Nicotine

\section{Exposure with pH1N1 Infection}

Alveolar mean linear intercept quantification within groups over time. In GraphPad Prism 7.03 two-tailed ttests compared to untreated controlled were implemented. Multiplicity adjusted p-values were reported for each comparison. Error bars correspond the standard deviation of the mean. Differences are represented at 210 days of age vs.34 days of age, $\mathrm{p}<0.001: * * *$. 
Further evaluation of the entire lung revealed some changes that are more difficult to explain. It was discovered that there were regions of inflammation/pneumonitis, which we believe are likely related to infection-induced pneumonitis (Fig. 28). The pneumonitis was characterized by edema, peri-airway collagen deposition, massive inflammation and consolidation throughout the parenchyma, within the airways. This phenotype was primarily occurring only in the transient nicotine with pH1N1 group (8 of 10 mice), although transient nicotine alone had one mouse and chronic nicotine with $\mathrm{pH} 1 \mathrm{~N} 1$ had two mice with this observed phenotype (Fig. 28). This abnormality was detected in a single to 3 lung lobes and primarily affected areas around large airways extending into the parenchyma in some instances. We estimate that up to $5-20 \%$ of the lung lobe was affected when present. Further, for transient nicotine with $\mathrm{pH} 1 \mathrm{~N} 1$ group in the mice where pneumonitis was not discovered, evidence of mild inflammation around central airways was discovered. We suspect this mild inflammation was involved in the resolution or development of the observed pneumonitis.

It is important to mention that for mean linear intercept quantification, both regions around the large airways as well as regions with viral pneumonia were avoided. Analyzing the distance between alveolar septum would be increased if large airways were present in representative images, due to having less area of the parenchyma present. Furthermore, viral related pneumonitis occurred in almost all instances around the large airway making avoidance of the abnormality easy. 

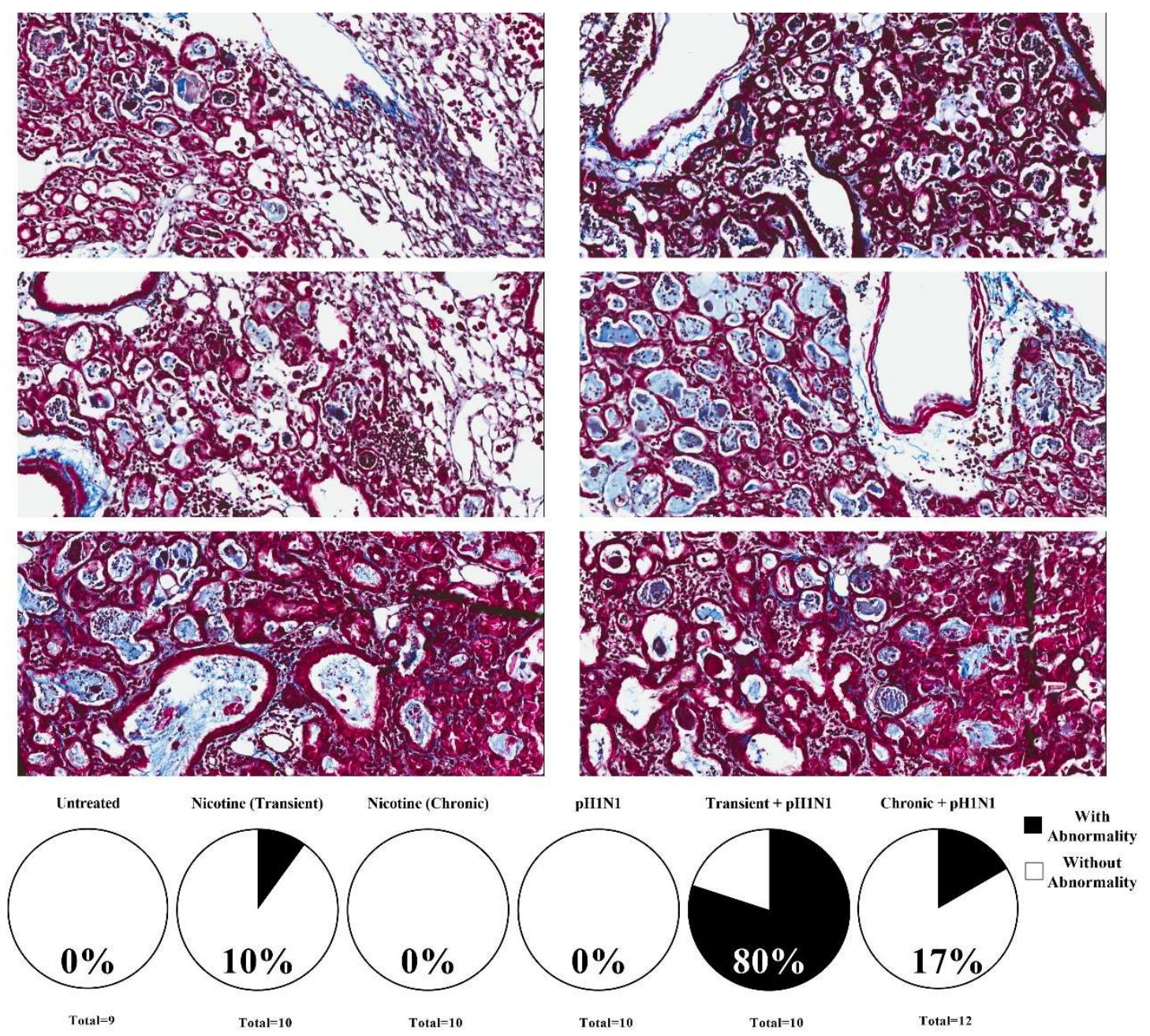

Figure 28. Histological Signs of Viral Related Pneumonitis in Transient Nicotine with pH1N1 at 210 Days of Age

Masson's trichrome staining representative images discovered in transient nicotine with pH1N1 infected group. Corresponding pie graphs for each treatment group and the resulting quantification of mice with this phenotypic abnormality. 


\section{$\underline{\text { Discussion }}$}

The effects of perinatal nicotine exposure alone have been studied and published in regard to lung function, but the impact of these events on aged animal models (like reported here) are not common. In contrast, studies regarding influenza infection resulting in lung function abnormalities are not common and not one has looked as far out in life as this study did (210-days of age). To our knowledge, this is the first study to look at the combination of nicotine exposure with influenza infection at any point of life on lung function. Importantly, our model used nicotine exposure (perinatal and chronic) and an early life infection with $\mathrm{pH} 1 \mathrm{~N} 1$. This is important and highly relevant to humans due to the number of pregnant mothers who continue to expose their fetus to nicotine (through cigarette smoking) and the number of children who contract an influenza respiratory infection (discussed above).

Although, cigarette smoking is the behavior linked to a number of consequences both for the fetus and the mother, nicotine is one constituent of tobacco that is responsible for its addictive qualities $(42,57,64)$. This plant alkaloid readily transverses the placenta, and fetal levels of nicotine or its metabolite cotinine are significantly greater than in the mother (discussed above) $(57,61)$. Our laboratory has studied how gestational, perinatal, or postnatal nicotine exposures impact the lung, and previously reported that murine maternal perinatal nicotine exposure affects lung branching morphogenesis, which was associated with airway dysfunction in the young animal and aged animal (8 weeks or 210days of age $)(42,64)$. This loss of lung function was postulated to be driven by alterations in extracellular matrix expression and deposition, among other changes, resulting in 
dysynaptic lung growth or alveolar destruction causing increased alveolar volume/lung compliance (Chapter II) (42).

Unlike typical seasonal IAV, H1N1 the $2009 \mathrm{pH} 1 \mathrm{~N} 1$ strain led to an increased rate of infection for individuals between $10-19$ years of age (15.6\% versus $25.1 \%$ of cases) $(156,157,181)$. Therefore, much of the population that became infected with the 2009 pH1N1 strain were middle to high school aged individuals, which correlates to the age of mice being used in our study. As shown in Chapter III, pH1N1 infection caused alterations in lung function in aged mice, which was driven by collagen deposition that occurred shortly after infection. However, the question remained whether early changes caused by nicotine followed by early life pH1N1 infection could result in worse structural and functional abnormalities in the adult lung.

We first evaluated the weights of animals as it is a good surrogate for lung growth, and effects on lung growth can affect its maturation. The average weights of the experimental groups were not different at the time of $\mathrm{pH} 1 \mathrm{~N} 1$ infection and pulmonary lung function testing (Fig. 21). However, as expected, weights early after infection for the three groups infected with $\mathrm{pH} 1 \mathrm{~N} 1$ showed statistical differences from the untreated group (Fig. 21). Interestingly, the weights of animals infected with $\mathrm{pH} 1 \mathrm{~N} 1$ alone or exposed to chronic nicotine followed by $\mathrm{pH} 1 \mathrm{~N} 1$ were not found to be different at any time post infection (up to $21 \mathrm{DPI}$ ). In contrast, transient nicotine with $\mathrm{pH} 1 \mathrm{~N} 1$ infection caused weights to significantly vary from the other infection groups (Fig. 21). Although animals with chronic nicotine exposure followed by $\mathrm{pH} 1 \mathrm{~N} 1$ infection experienced the same change in percent body weight from time of infection as $\mathrm{pH} 1 \mathrm{~N} 1$ alone, the functional pulmonary outcomes were not the same (Fig. 22). 
Considering previous findings with nicotine alone (Chapter II), it was expected that chronic nicotine exposure followed by $\mathrm{pH} 1 \mathrm{~N} 1$ infection would further impact lung function in the aged animal. Interestingly, there were limited functional alterations (compared to untreated-mock infected). It appeared that the changes discovered for the combination were driven by $\mathrm{pH} 1 \mathrm{~N} 1$ infection alone, due to these parameters (resistance and tissue damping) not being affected by chronic nicotine by itself (Fig. 22) (summarized in Table 10). This proves that the change in weight acutely due to $\mathrm{pH} 1 \mathrm{~N} 1$ is not solely responsible for the pulmonary functional abnormalities detected. The alterations in lung structure (collagen and alveolar size) for animals with the combination of chronic nicotine exposure followed by $\mathrm{pH} 1 \mathrm{~N} 1$ infection were not different from the untreated group, with the exception of females (alveolar size) (Fig. 23). Not seeing phenotypical alterations in mean linear intercept provides rationale as to why compliance, elastance, and tissue elastance were unchanged. Further, no evidence of differences in collagen staining provides rationale for why Newtonian resistance was not abnormal (from untreated controls). We expect alterations arose in resistance were attributed to parenchymal AHR as tissue damping was the only other functional parameter affected (Fig. 22).

In contrast to the above, transient nicotine exposure followed by pH1N1 infection resulted in significant and surprising functional and phenotypical alterations (Fig. 24\&25) (Table 11). Neither factor alone caused an increase in alveolar size or compliance. Whereas, mean linear intercept (alveolar size), but not collagen deposition, was increased for the combination which corroborates our functional findings for compliance, elastance, and tissue elastance (Fig. 25). However, pH1N1 infection (but not transient nicotine exposure) was found to cause changes in elastance and tissue elastance, which suggests 
that elastin in the parenchyma was destroyed, although more thorough analysis will need to be conducted to prove this. Furthermore, $\mathrm{pH} 1 \mathrm{~N} 1$ alone significantly increased collagen deposition (compared to untreated) which was most notably around the large airways. Since we saw no evidence of increased collagen deposition in transient nicotine with pH1N1 infection we expect that the functional abnormalities for resistance and Newtonian resistance may be attributed to increased AHR. Another explanation for increasing resistance and Newtonian resistance could be due to parenchymal destruction (increased alveolar size). Increased alveolar size (alveolar septum destruction) would decrease the structural connections to the airways which could in theory cause collapse of the airway and thus increase Newtonian resistance, and possible tissue damping as we discovered (Fig. 24).

In emphysema, it is well established that neutrophils are present in increased quantity and are thought to drive disease pathogenesis $(36,251)$. Since the 1980 's, nicotine alone has been known to enhance neutrophil responsiveness to alveolar macrophage chemotactic factors and thus increase their quantity in the lung (252). In addition to nicotine, pH1N1 is known to cause a strong neutrophil response in the lung $(75,77,201)$. Separately, both nicotine and $\mathrm{pH} 1 \mathrm{~N} 1$ alone can increase lung neutrophil concentration and may together potentiate or prolong the effects. Neutrophil infiltration could increase MMP9 expression (which is known to degrade collagen, among other actions) and elastase, which is crucial for alveolar septation $(253,254)$. Further, nicotine alone has also been published to increase the expression of neutrophil elastase, another matrix degrading enzyme with elastolytic activity (255). Together, this may explain the observed alveolar volume increase in the lung, although more thorough testing will need to be conducted in 
order to prove this speculation correct. Altogether, these data show that the lung is being affected in additional ways with transient nicotine with $\mathrm{pH} 1 \mathrm{~N} 1$ versus $\mathrm{pH} 1 \mathrm{~N} 1$ alone.

Due to the findings for transient nicotine with $\mathrm{pH} 1 \mathrm{~N} 1$ but not chronic nicotine with $\mathrm{pH} 1 \mathrm{~N} 1$ we wanted to look at the lungs of these groups at an earlier timepoint (34-days of age) (Fig. 25). We only evaluated mean linear intercept due to not seeing differences in collagen deposition in the long term. Furthermore, we only evaluated the combination group compared to untreated group due to not seeing differences in either entity alone at 210-days of age (Fig. 9). Alveolar septa destruction is unrepairable and thus if it was present for $\mathrm{pH} 1 \mathrm{~N} 1$ or transient nicotine alone, early in life (34-days of age) it would be present later in life (210-days of age), which was not found (Fig. 25). When evaluating transient nicotine with pH1N1 at 34-days of age (4 DPI) we found that the mean linear intercept or alveolar size was increased compared to untreated mice (Fig. 26). This finding provides insight in that the key stage for alveolar destruction within our murine model was occurring between 30 and 34-days of age, which is within the timeframe in which pH1N1 viral lung propagation is at its height (Fig. 12). Furthermore, this destruction continues to occur as the animal ages to 210-days of age as we found differences in alveolar volume between the two timepoints (Fig. 27).

Lastly, it is important to emphasize that the data need to be confirmed in other studies. In particular, we are concerned that some of these animals suffered pneumonitis, perhaps infectious, at the end of the experiment, which likely impacted the pulmonary function tests (Fig. 28). We were careful not to evaluate areas of the lung that contained these abnormalities when performing mean linear intercept analysis. Avoiding areas of viral related pneumonitis was not an issue when the left lobe was not affected. In the few 
instances where the left lobe was affected, the areas where pneumonitis was occurring were around the large central airways which we avoided as before. However, we cannot discard the possibility that they affected our functional results. We believe that both the increase in alveolar volume as well as the viral related pneumonitis occurring were causing alterations in respiratory function.

In humans, the combination of the exposures we elicited in mice have been suggested through epidemiological studies involving children to be implicated in the development of OAD both in childhood and adulthood (131, 256-260). Importantly, the evidence in humans is purely speculative because there are limited studies conducted to address the relationship of perinatal nicotine with early life viral infection. Within this chapter, we provide information that may explain data linking these events $(46,47,67-$ $70,72,124,126,130-132,174,175,178)$. Altogether, the data generated strengthen the argument that events early in life (transient nicotine exposure) may influence the lung in imperceptible ways, that prime the tissue to be further irreversibly damaged by a second hit (such as $\mathrm{pH} 1 \mathrm{~N} 1$ ). Although future experiments will need to be conducted in order to further explain the findings or lack thereof (chronic nicotine with $\mathrm{pH} 1 \mathrm{~N} 1$ ). This chapter provides the evidence that maternal nicotine exposure with an early life respiratory infection influence the lung to develop abnormalities which persist with age. 
CHAPTER V: PULMONARY FUNCTIONAL ABNORMALITIES SEEN IN ANIMALS EXPOSED TO NICOTINE WITH PH1N1 INFECTION ARE ALLEVIATED IN $\alpha 7$ NACHR DEFICIENT MICE 


\section{$\underline{\text { Background }}$}

The effects of early life exposure to nicotine and OAD development are likely mediated via one or more nicotinic acetylcholine receptors. Since the identification of these receptors might unveil important targets for intervention, we set out to investigate their role. There are two types of AChR, nicotinic (ion gated) and muscarinic (g-protein coupled) both of which are triggered by the endogenous agonist acetylcholine (58-60). The primary exogenous agonist that binds muscarinic $\mathrm{AChR}$ is muscarine, whereas nicotine primarily activates the $\mathrm{nAChR}(58,59)$. nAChR are a family of multimeric acetylcholinetriggered cation channel proteins that form the predominant excitatory neurotransmitter receptors in muscles and nerves in the peripheral nervous system (58-60). The nAChR are coded by at least thirteen genes that express $4 \beta$ subunits and $9 \alpha$ subunits, in which they assemble into pentamers in a homomeric or heteromeric orientation $(58,59)$. The most abundant homomeric nAChR in the human body is $(\alpha 7)_{5}$, also known as, $\alpha 7 \mathrm{nAChR}(261$, 262).

$\mathrm{nAChR}$ are found in the autonomic and central nervous systems throughout the body (58-60). There has also been published data showing that nAChRs are expressed in non-neuronal tissues including lung, immune cells, retinal cells and 3T3 fibroblasts (NIH) (263-266). Zia et al. used indirect immunofluorescence to show the presence of $\alpha 3, \alpha 5$ and $\alpha 7 \mathrm{nAChR}$ in human and mouse bronchial epithelial cells and further detected $\alpha 7 \mathrm{nAChR}$ in submucosal glands and $\alpha 4 \mathrm{nAChR}$ in alveolar epithelial cells (262). Expression of $\alpha 7$ $\mathrm{nAChR}$ in bronchial epithelial cells was increased in a dose dependent manner to nicotine, whereas $\alpha 3 \mathrm{nAChR}$ and $\alpha 5 \mathrm{nAChR}$ decreased only after nicotine desensitization (262). Our laboratory has shown that $\alpha 7 \mathrm{nAChR}$ expression in the C57B1/6 fetus's lung begins at 
day 11 of gestation and shows highest expression at days 12 and 15 of gestation (64). Expression of the $\alpha 7 \mathrm{nAChR}$ is decreased for the remainder of gestation (64). Therefore, maximal expression of $\alpha 7 \mathrm{nAChR}$ occurs during the pseudoglandular stage of embryonic lung development in the $\mathrm{C} 57 \mathrm{~B} 1 / 6$ mouse, coinciding with the period of airways formation (267).

Through poorly elucidated mechanisms of action, nicotine has been demonstrated by our laboratory to affect lung branching morphogenesis in murine offspring exposed to nicotine during the perinatal period (both in vivo and ex vivo) $(42,64)$. These changes were associated with increased extracellular matrix deposition, abnormal airway structure, and AHR. Interestingly, these changes were not apparent in animals lacking the $\alpha 7 \mathrm{nAChR}$ indicating an important role for this receptor in mediating the effects of nicotine $(42,64)$. In addition, nicotine was shown to promote fibroblast ECM synthesis, through activation of TGF $\beta$ and the $\alpha 7 \mathrm{nAChR}$ were found to mediate the effects $(196,268)$. Similar to our published data, Elliot Spindel's group has published in a non-human primate prenatal nicotine exposure model that nicotine lowers offspring lung function through increasing airway resistance (63). This work also showed increased $\alpha 7 \mathrm{nAChR}$ expression in the airways of the offspring, and increased collagen deposition around the large airways and vessels (62). These models are in line to what was found in a human study assessing pulmonary abnormalities from cases of fetal or newborn deaths (269). This study published that fetal and newborn deaths, where maternal smoking was documented, showed increased $\alpha 7 \mathrm{nAChR}$ immunostaining in the lung as well as lung hypoplasia (269).

Overall, these data and observations presented throughout this dissertation suggest that early life events (such as but not limited to perinatal nicotine exposure) render the 
offspring susceptible to the development of airway dysfunction and OADs. Importantly, they point to $\alpha 7 \mathrm{nAChR}$ as a potential mediator of these effects. Herein, we investigate the role of a $7 \mathrm{nAChRs}$ and report the effects of perinatal (transient) with pH1N1 infection on PFTs and resulting lung histology in wild type (WT) and $\alpha 7 \mathrm{nAChR}$ deficient mice. We show that the absence of the $\alpha 7 \mathrm{nAChR}$ leads to alleviation of PFT abnormalities for perinatal nicotine exposure with $\mathrm{pH} 1 \mathrm{~N} 1$, but not for any entity alone. This was associated with less alterations in alveolar structure. All together, these studies further support a role for $\alpha 7 \mathrm{nAChRs}$ and suggests attention should be given to these receptors as potential targets for therapeutic intervention.

\section{Materials and Methods}

Materials and methods for this section are the same as presented in the previous chapters, with the following sections being changed. Statistics were conducted for PFT data with the baseline and $50 \mathrm{mg} / \mathrm{mL}$ methacholine exposure. Mice deficient for the $\alpha 7$ nAChR were ordered from Jackson Laboratories (\#003232) and lacked 3 exons (8-10) in the gene spanning $7 \mathrm{~kb}$ (270). Both in our study and previous studies, $\alpha 7 \mathrm{nAChR}$ deficient mice had no abnormalities compared to wild type for growth, neurological deficits, and overall appearance (270). For PFT data one-way ANOVA analysis with a Bonferroni test to correct for multiple comparisons as well as two tailed unpaired $\mathrm{T}$ tests (with similar results) were implemented in GraphPad Prism 7.03. All other analysis were conducted as before in Chapters II-IV. 


\section{$\underline{\text { Results }}$}

Transient Nicotine Exposure followed by pH1N1 infection in $\alpha 7$ nAChR Deficient Mice

Animal weights were examined as they represent a good surrogate for lung growth. Untreated WT and $\alpha 7 \mathrm{nAChR}$ deficient mice showed appropriate and similar weight gain over time and up to 21 DPI. (Fig. 29). As expected, pH1N1 infection resulted in significant weight loss, which was greatly worsened in animals concomitantly exposed to nicotine (321 DPI). Whereas, significant differences (decrease in weight gain) were detected later (8$10 \mathrm{DPI})$ in $\alpha 7 \mathrm{nAChR}$ deficient mice (Fig. 29). In addition, differences occurred between WT and $\alpha 7 \mathrm{nAChR}$ deficient mice in the transient nicotine exposure with $\mathrm{pH} 1 \mathrm{~N} 1$ infection group from 8-11 DPI (with WT being more affected by infection). Furthermore, no differences were discovered in raw weight between groups at 30-days of age (Fig. 29). In contrast, at 210-days of age there was a difference between WT and $\alpha 7 \mathrm{nAChR}$ deficient mice exposed to transient nicotine with $\mathrm{pH} 1 \mathrm{~N} 1$ infection (with $\alpha 7 \mathrm{nAChR}$ deficient mice weighing more). 


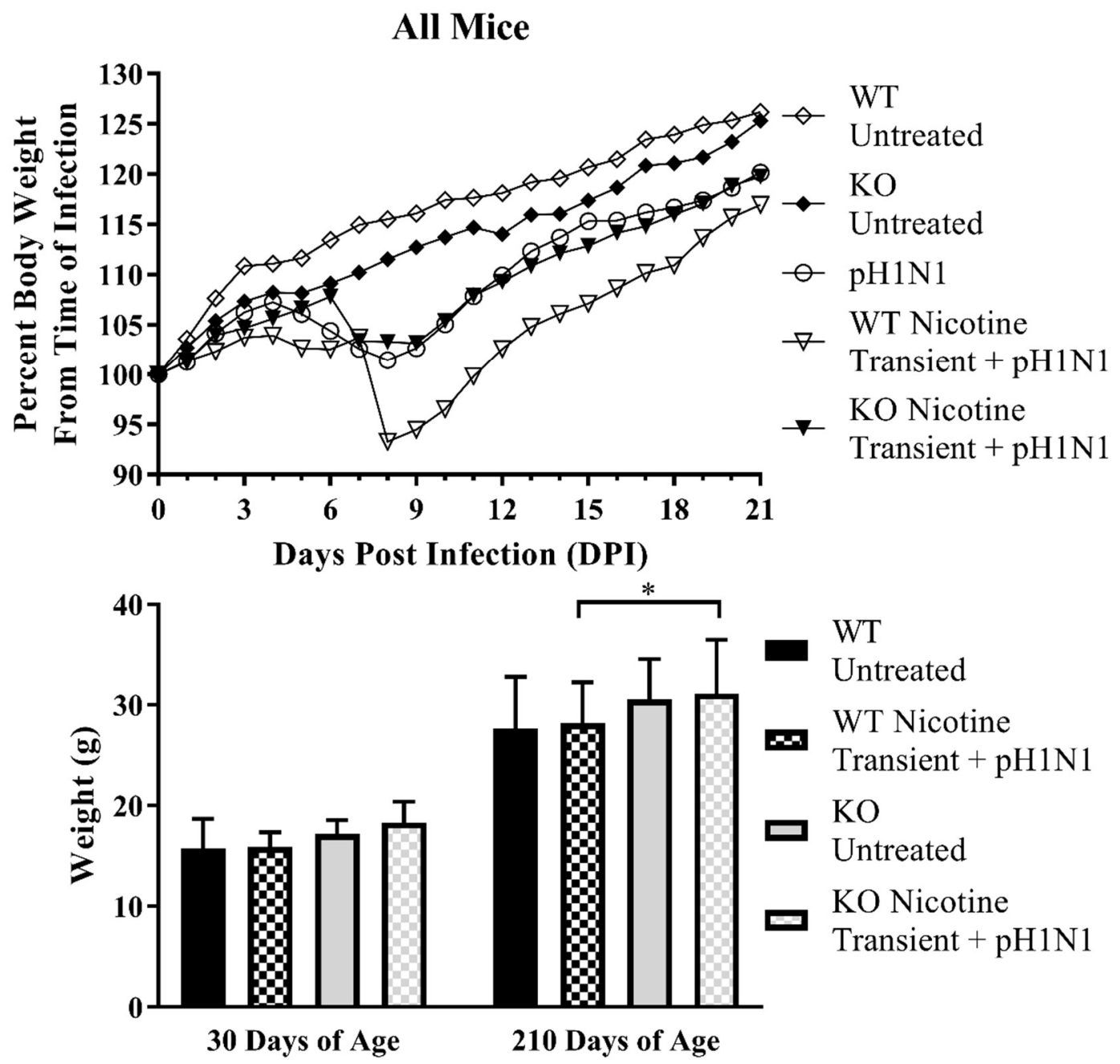

Figure 29. Weights of Transient Nicotine Exposed Mice with pH1N1 Infection (Wild

\section{Type and $\alpha 7$ nAChR Deficient) Assessed for Lung Functional Abnormalities}

Average percent body weights (30 until 51-days of age) and average weights (30 and 210-days of age) of mice groups for the indicated exposures. Data was imported into GraphPad Prism 7.03, the average daily weights and percent from time of infection weights were calculated along with their standard deviations then compared using two-way ANOVA analysis with a Turkey post-test to correct for multiple comparisons (with no differences discovered). The weights at 30 and 210-days of age were compared using unpaired t-test analysis with a Holm-Sidak multiple comparison test. A linear regression analysis was also conducted between groups in GraphPad, with no differences reported. 
Lung Function in Nicotine Exposure followed by pH1N1 infection in WT and $\alpha 7 n A C h R$ Deficient Mice

We performed PFT in WT mice and mice deficient for the $\alpha 7 \mathrm{nAChR}$. No differences were discovered (at baseline or $50 \mathrm{mg} / \mathrm{mL}$ methacholine separately) between WT and $\alpha 7 \mathrm{nAChR}$ deficient mice groups for transient nicotine, chronic nicotine exposure, or pH1N1 infected groups alone (data not shown). Likewise, PFT findings in untreated $\alpha 7$ nAChR deficient mice mimicked those in untreated WT mice (data not shown). When assessing the PFT results at $50 \mathrm{mg} / \mathrm{mL}$ methacholine (using GraphPad), resistance, tissue damping, and elastance were significantly increased in WT animals with transient nicotine exposure followed by pH1N1 infection (compared to WT untreated-mock infected animals). However, these alterations were essentially abolished in $\alpha 7 \mathrm{nAChR}$ knockout animals suggesting that $\alpha 7$ nAChRs mediated many of the effects observed (Fig. 30).

Lung Histology in Nicotine Exposure followed by pH1N1 infection in WT and $\alpha 7 n A C h R$ Deficient Mice

In WT mice, transient nicotine exposure followed by $\mathrm{pH} 1 \mathrm{~N} 1$ infection resulted in increased alveolar mean linear intercept (compared to WT untreated-mock infected animals), and this effect was significantly blunted by in $\alpha 7 \mathrm{nAChR}$ deficient animals (Fig. 31 A\&C). Transient nicotine exposure followed by pH1N1 infection did not affect collagen deposition in either group of animals (Fig. 31 B\&D). As discussed in the previous chapter, some animals developed signs of pneumonitis, probably infectious. However, signs of viral related pneumonitis were not present in $\alpha 7 \mathrm{nAChR}$ deficient mice 
exposed to transient perinatal nicotine with $\mathrm{pH} 1 \mathrm{N1}$ (Fig. 32). Nevertheless, this could have impacted the analysis. 

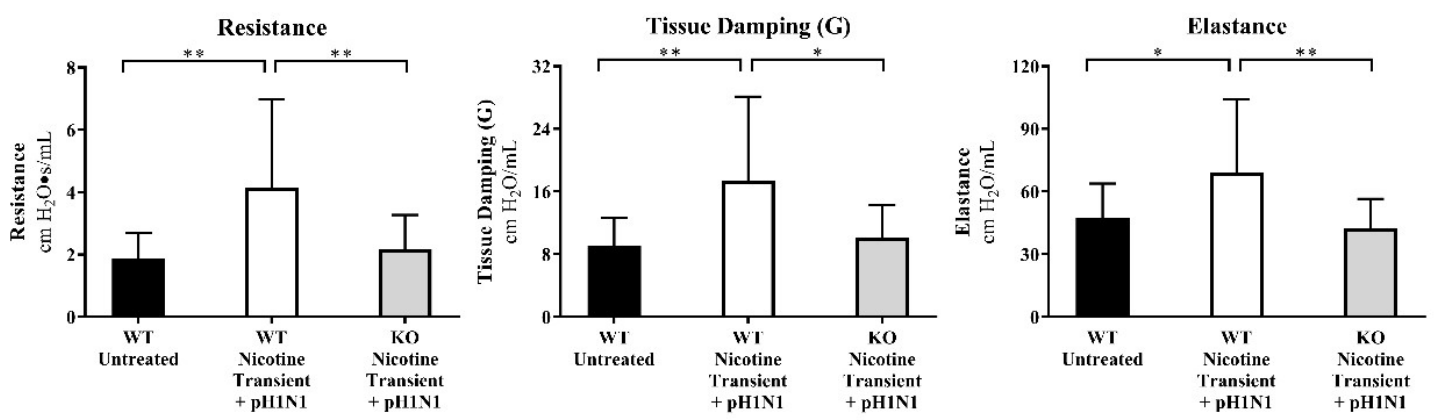

Figure 30. Pulmonary Function Test Results for Transient Nicotine with pH1N1

\section{Infection in $\alpha 7 \mathrm{nAChR}$ Deficient Mice}

Lung function was assessed at 210-days of age by forced oscillation technique using a SCIREQ flexiVent system. C57BL/6 Untreated (mock infected) mice $(\mathrm{n}=19 ;$ male $=10$, female $=9)$ and mice in the transient nicotine with $\mathrm{pH} 1 \mathrm{~N} 1$ infection group $(\mathrm{n}=20$; male $=10$, female $=10)$ were tested. $\alpha 7 \mathrm{nAChR}$ deficient $(\mathrm{KO})$ untreated (mock infected) mice $(\mathrm{n}=15$; male $=7$; female $=8)$ and mice in the transient nicotine with pH1N1 infection group $(n=12$; male $=6$; female $=6)$ were tested. Lung function was measured at baseline and after challenge with increasing concentrations of aerosolized methacholine. All statistics were conducted with the $50 \mathrm{mg} / \mathrm{mL}$ methacholine exposure data. In GraphPad Prism 7.03 one-way ANOVA analysis with Bonferroni correction for multiple comparisons as well as two-tailed unpaired non-parametric t-tests comparing all groups were implemented. Averages of results (only including values where the COD was $\geq 95 \%$ ) were taken for each individual mouse. Error bars represent standard deviation from the mean. Differences are represented as, $\mathrm{p}<0.05:^{*}, \mathrm{p}<0.01: * *$. 

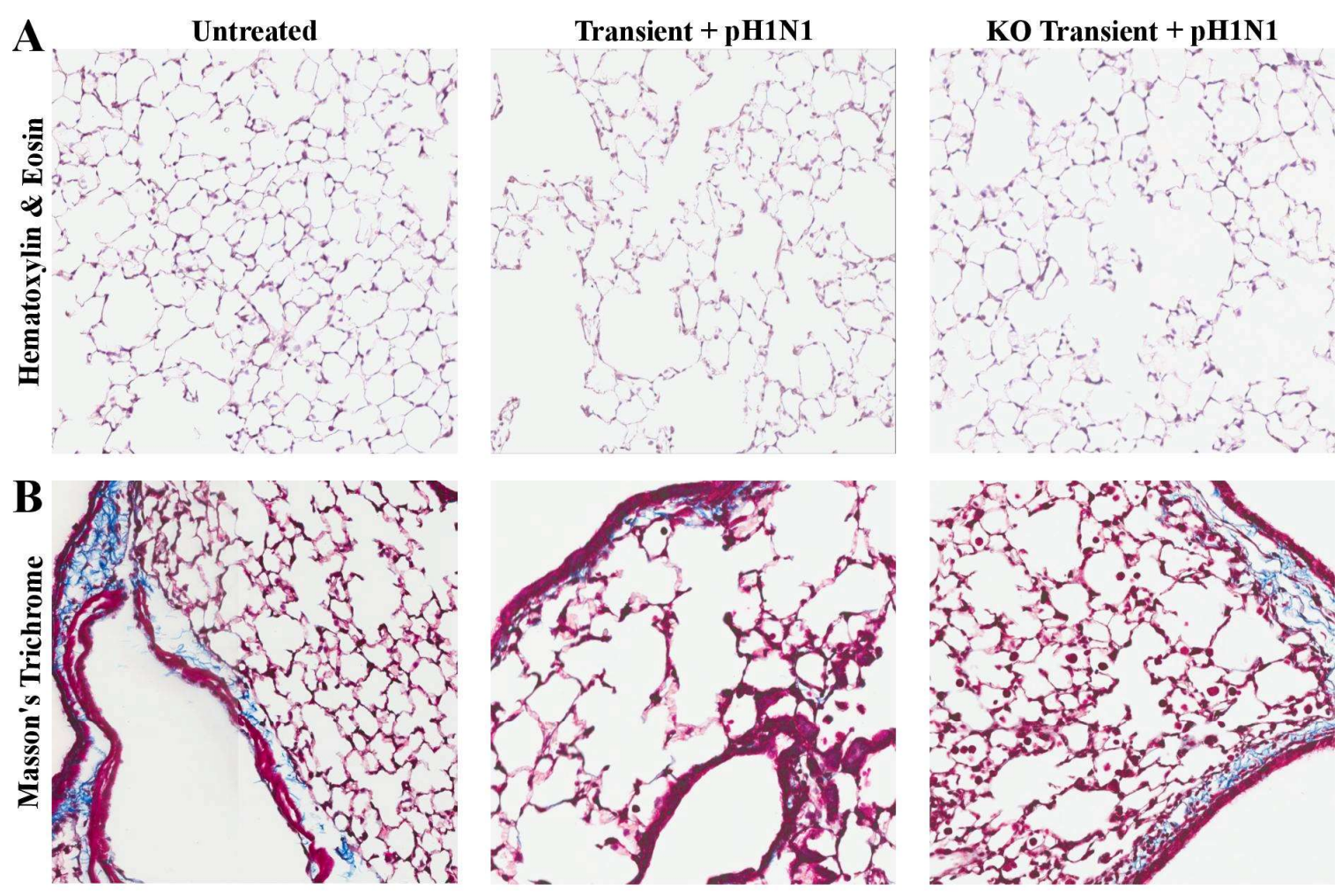

$\mathrm{C}$

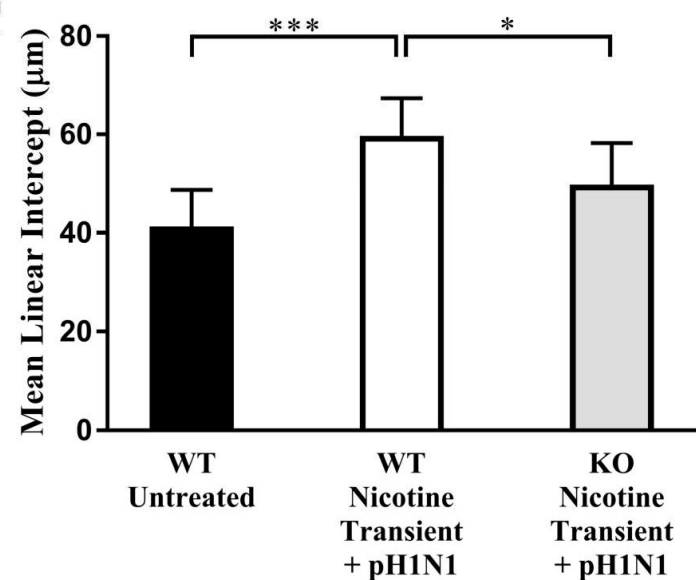

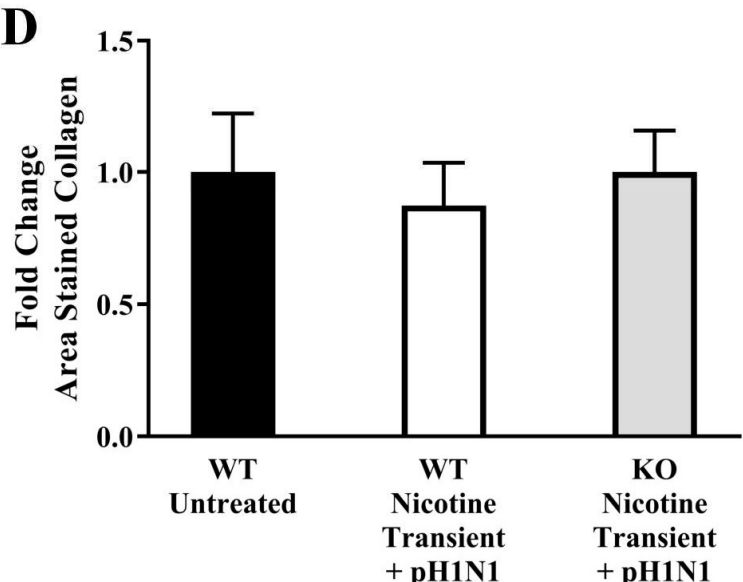

Figure 31. Lung Histology of Transient Nicotine Exposure with pH1N1 Infection at

\section{0-Days of Age for $\alpha 7$ AChR Deficient Mice}

Lung histology in C57BL/6 mice, including H\&E staining at 20x magnification (A) and Mason's Trichrome staining at 20x magnification (B). Alveolar mean linear intercept quantification (C) and the percent of total lung area stained for collagen (D). In GraphPad Prism 7.03 one-way ANOVA analyses were conducted with all groups using a Bonferroni test to correct for multiple comparisons. Multiplicity adjusted p-values were 
reported for each comparison. Error bars correspond the standard deviation (C) or error (D) the mean. Differences are represented vs. mock infected controls, $\mathrm{p}<0.05$ : $^{*}, \mathrm{p}<0.001$ : ***. 


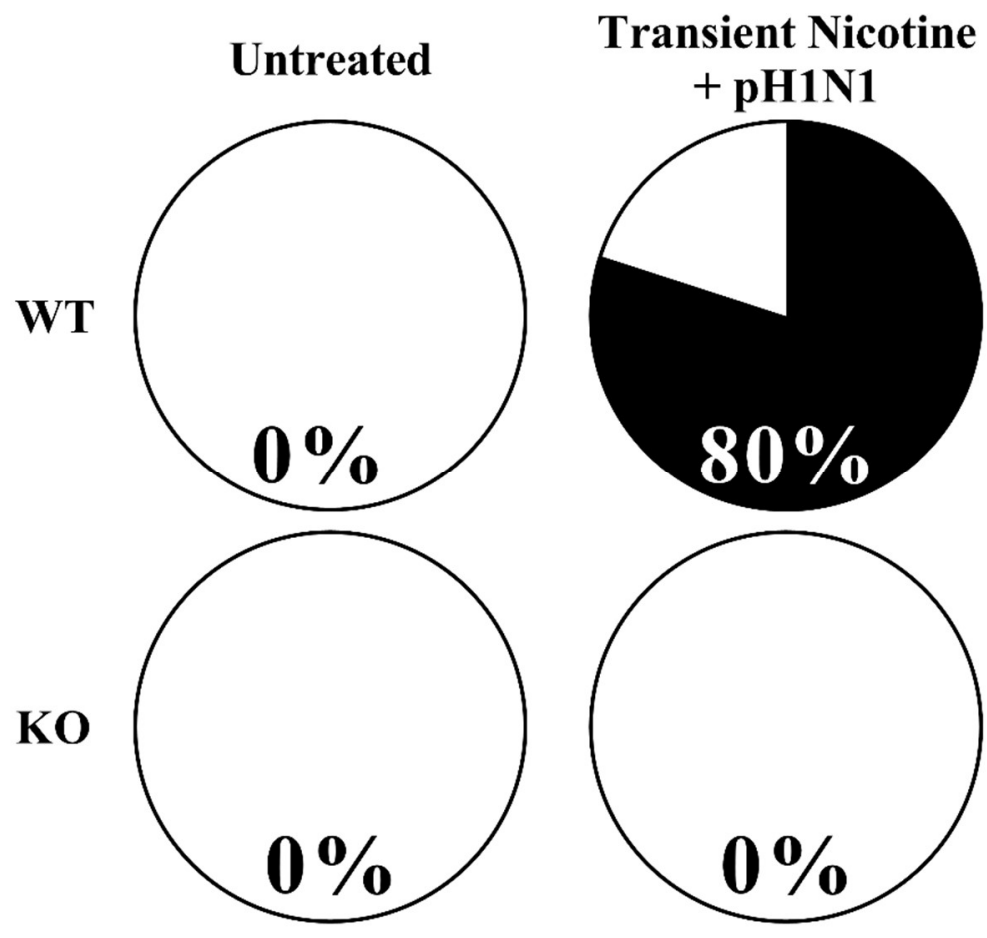

Figure 32. Histological Signs of Viral Related Pneumonia in Transient Perinatal Nicotine Not Seen in $\alpha 7$ nAChR Deficient Mice

Quantification of mice in each treatment group evaluated for pneumonia via Masson's trichrome staining for both wildtype and $\alpha 7 \mathrm{nAChR}$ deficient mice. 


\section{$\underline{\text { Discussion }}$}

Our studies show transient nicotine exposure followed by $\mathrm{pH} 1 \mathrm{~N} 1$ infection is associated with weight loss and alterations in lung structure and function. Interestingly, and consistent with our hypothesis, these changes were greatly ameliorated or abolished in the $\alpha 7 \mathrm{nAChR}$ knockout mice suggesting an important role for this receptor during perinatal transient nicotine with early life $\mathrm{pH} 1 \mathrm{~N} 1$ infection. Interestingly, chronic nicotine exposure had different effects suggesting that lack of $\alpha 7 \mathrm{nAChR}$ worsened the impact of the exposures. However, these latter experiments were greatly affected by the fact that the animals were found to have significant structural changes at 210-days of age, most likely related to the development of pneumonia. We do not know if $\alpha 7 \mathrm{nAChR}$ deficiency leads to predisposition to lung infection and thus, at this time, we cannot interpret these studies. Thus, we will focus our discussion on the data obtained in animals with nicotine transient exposure with $\mathrm{pH} 1 \mathrm{~N} 1$ infection.

Also note that when assessing mice exposed to transient perinatal nicotine with pH1N1 infection, $\alpha 7$ nAChR deficiency was determined to alleviate abnormalities in resistance, tissue damping, and elastance (Fig. 30). It is important to mention that the analysis conducted with the $\alpha 7 \mathrm{nAChR}$ deficient mice was conducted only with the data resulting at baseline (no differences discovered) and $50 \mathrm{mg} / \mathrm{mL}$ methacholine, whereas before (Chapters II-IV), an analysis was conducted using baseline, 25, and $50 \mathrm{mg} / \mathrm{mL}$ methacholine exposures.

Previously, we reported alterations in collagen deposition after perinatal (transient) nicotine exposure (42). However, when examining animals at 210-days of age, we found that these defects did not persist until adulthood. However, transient nicotine with $\mathrm{pH} 1 \mathrm{~N} 1$ 
resulted in abnormalities in pulmonary function and alveolar structure suggesting that the combined exposures affected this process in ways that involve cholinergic signaling through $\alpha 7$ nAChRs.

We encountered evidence of pneumonitis in some of our animals. Although we were careful to avoid such areas when evaluating lung structure, we cannot discard the possibility that these changes affected lung function. Again, areas of pneumonitis were not present throughout the lung, but primarily localized around large airways (for WT mice). These regions were avoided when conducting mean linear intercept quantitation. Interestingly, $\alpha 7 \mathrm{nAChR}$ deficient mice exposed to transient perinatal nicotine with $\mathrm{pH} 1 \mathrm{~N} 1$ did not show histological signs of pneumonitis as seen in the wildtype, but we cannot determine if this relates to their genetic background (Fig. 34).

It appears from our results that the $\alpha 7 \mathrm{nAChR}$ interaction with nicotine from our transient nicotine exposure primes the lung for further damage from a viral agent such as pH1N1. We would expect that other environmental or occupational exposures would result in similar findings but more experiments will need to be conducted as this is merely speculation. These finding are vital in translating this research into treating humans during early/before life nicotine exposure, in order to protect one from an OAD later in life. If one is subject to perinatal nicotine exposure, protection to an OAD could be achieved through administration of a $\alpha 7 \mathrm{nAChR}$ antagonist such as conotoxin or bungarotoxin to the mother (this is assuming one will have a viral infection in adolescence) (271). These data suggest that by inhibiting $\alpha 7 \mathrm{nAChR}$ signaling for persons with perinatal, but not chronic nicotine exposure, with an early life viral infection could prevent or prolong the development of an OAD to occur with aging. 


\section{CHAPTER VI: DISCUSSION}


The objective of this research was to determine if perinatal, and/or early life exposures in a murine model promote lung structural and functional alterations in the adult, thereby mimicking what is seen in humans with OAD. We initially hypothesized that nicotine exposure and $\mathrm{pH} 1 \mathrm{~N} 1$ infections during would alone or in concert act to promote the development of sustained airway dysfunction in adulthood. This is indeed what we observed (Chapter II and III) (Table 12). The effects of transient perinatal nicotine resulted in no functional abnormalities suggesting AHR did not persist with aging (Table 12), whereas, chronic nicotine drove functional alterations through increasing compliance, decreasing Newtonian resistance, and decreasing tissue elastance (Table 12). Nicotine drove these alterations by enlarging alveolar spaces and increasing peri-airway collagen deposition as well as alveolar simplification, and destruction with aging (Chapter II). Similarly, pH1N1 infection was also capable of causing lung structural (collagen deposition) and functional abnormalities (increased resistance, tissue damping, and tissue elastance) that persisted until adulthood (increased resistance, tissue damping, and tissue elastance) (Chapter III) (Table 12). Interestingly, differences observed between chronic nicotine versus pH1N1 were apparently due to different regions of the airway being affected, which was most likely due to the differences in structure observed compared to untreated-mock infected (increased alveolar volume and collagen deposition for chronic nicotine alone versus increased collagen deposition for $\mathrm{pH} 1 \mathrm{~N} 1$ alone) (Table 12). For chronic nicotine alone, collagen deposition around the large airways may have increased the rigidity of the airways and kept them from collapsing due to parenchymal destruction (hence the lack of increase in Newtonian resistance). 
Together, transient perinatal nicotine exposure along with early life pH1N1 infection potentiated the effects of either entity alone (Chapter IV) (Table 12). In regard to nicotine exposure alone, alterations in lung function and structure were observed for chronic nicotine but not for transient nicotine treatment. More specifically, transient nicotine in combination with $\mathrm{pH} 1 \mathrm{~N} 1$ was able to potentiate the outcomes for either factor alone (resistance, compliance, and Newtonian resistance) (Fig. 24). Further, transient nicotine with $\mathrm{pH} 1 \mathrm{~N} 1$ caused alterations that were not seen in either entity alone (compliance, elastance, and Newtonian resistance), which we found were associated with increased alveolar volume (Table 12). Interestingly, chronic nicotine in combination with pH1N1 was found to alleviate some of the abnormalities discovered for each exposure alone (compliance, elastance, Newtonian resistance, and tissue elastance) (Fig. 22) (Table 12). We speculate that immune response signaling due to $\mathrm{pH} 1 \mathrm{~N} 1$ was driving this, although additional experiments would have to be conducted to test this.

It appears that transient nicotine exposure is priming the lung in a way for $\mathrm{pH} 1 \mathrm{~N} 1$ infection to cause irreversible alveolar septum damage, which results in lung functional abnormalities, that is worse than either entity alone. Furthermore, this provides support for the concept that the critical period of murine pulmonary alveolar septum destruction occurs on or after 30-days of age (when pH1N1 was elicited). When assessing $\alpha 7 \mathrm{nAChR}$ deficient mice (Chapter V) exposed to transient nicotine exposure with $\mathrm{pH} 1 \mathrm{~N} 1$ infection, pulmonary function deficiencies were alleviated for resistance, tissue damping, and elastance (Fig. 30). The data generated within this dissertation corroborate our laboratory's and other published findings on lung function and OADs regarding nicotine exposure, early life viral infections, the combination of perinatal nicotine with $\mathrm{pH} 1 \mathrm{~N} 1$, and the $\alpha 7 \mathrm{nAChR}$. 
Table 12. Pulmontary Fucntion Testing Summary

\begin{tabular}{|c|c|c|c|c|c|c|}
\hline & Resistance & Compliance & Elastance & $\begin{array}{l}\text { Newtonian } \\
\text { Resistance }\end{array}$ & $\begin{array}{c}\text { Tissue } \\
\text { Damping }\end{array}$ & $\begin{array}{c}\text { Tissue } \\
\text { Elastance }\end{array}$ \\
\hline $\begin{array}{l}\text { Nicotine } \\
\text { Transient }\end{array}$ & $\begin{array}{c}\text { No } \\
\text { Change }\end{array}$ & No Change & $\begin{array}{l}\text { No } \\
\text { Change }\end{array}$ & No Change & $\begin{array}{c}\text { No } \\
\text { Change }\end{array}$ & $\begin{array}{c}\text { No } \\
\text { Change }\end{array}$ \\
\hline $\begin{array}{l}\text { Nicotine } \\
\text { Chronic }\end{array}$ & $\begin{array}{l}\text { No } \\
\text { Change }\end{array}$ & 个 & $\begin{array}{l}\text { No } \\
\text { Change }\end{array}$ & & $\begin{array}{l}\text { No } \\
\text { Change }\end{array}$ & \\
\hline pH1N1 & 个 & No Change & $\begin{array}{l}\text { No } \\
\text { Change }\end{array}$ & No Change & 个 & $\uparrow$ \\
\hline $\begin{array}{l}\text { Transient } \\
+ \text { pH1N1 }\end{array}$ & $\uparrow$ & $\uparrow_{B}$ & B & 个 & 个 & 个 \\
\hline $\begin{array}{l}\text { Chronic } \\
+ \text { pH1N1 }\end{array}$ & 个 & No Change & $\begin{array}{l}\text { No } \\
\text { Change }\end{array}$ & No Change & 个 & $\begin{array}{c}\text { No } \\
\text { Change }\end{array}$ \\
\hline \multicolumn{7}{|c|}{$\begin{array}{l}\text { Arrows indicate direction of parameter compared to untreated-mock infected control } \\
\text { Blue indicates alteration in function driven by males } \\
\text { Black indicates alteration in function not driven by either sex } \\
\text { "B" indicates baseline }\end{array}$} \\
\hline
\end{tabular}




\section{$\underline{\text { Strengths and Limitations of Analysis }}$}

The data generated for all treatment groups had both strengths and caveats. Strengths for this project were that large numbers of mice were used within each treatment groups, with relatively equal numbers between sex type. In addition, statistical expertise for the functional data sets was obtained through collaboration with University of Louisville's biostatistician department (Dr. Shesh Rai and Jayesh Rai). Therefore, we are confident that the functional analysis and resulting data accurately represent what was occurring between our treatment groups. Furthermore, models for nicotine exposure and pH1N1 infection were well developed and characterized for their use in murine studies.

Limitations for the data presented within this dissertation are within determining where pH1N1 was replicating and histological analysis. Regarding pH1N1, we did not evaluate where the viral infection was occurring in the lung. Although we did see high levels of virus through conducting $\mathrm{TCID}_{50} / \mathrm{mL}$ assays on lung homogenate from infected animals, knowing the location would have provided more insight into the functional abnormalities. In retrospect, immunohistochemistry against pH1N1 could have been implemented to determine viral replication within the lung. Additionally, histological analysis for mean linear intercept measures airspace volume by assuming shape factor (141). Distortion of airspace and alveolar ducts can invalidate the assumption in shape factor (141). Further, mean linear intercept does not take into account alveolar septum thickness, which could have impacted our analysis, although, we did not see prominent increases in alveolar septum thickness (141).

Finally, we identified areas of pneumonitis in some of the lung samples harvested during our final experiments, especially those related to transient nicotine with $\mathrm{pH} 1 \mathrm{~N} 1$ 
infection. To our knowledge, no published study has shown this abnormality from either nicotine or $\mathrm{pH} 1 \mathrm{~N} 1$ alone. The development of pneumonitis (suspected to be infectious) was likely involved in the functional abnormalities observed. Interestingly, other groups did not show this phenotype as prominently. Considering the potential impact of pneumonitis on our functional data, it is recommended that these studies be confirmed in future experiments.

\section{$\underline{\text { Future Directions }}$}

\section{Different Viral Families and Different Age of Infection}

To our knowledge, this is the first study to our knowledge to look at the effects of perinatal nicotine and early life influenza infection on lung remodeling and pulmonary function and their effects long term. In which, our work has raised an additional series of questions. For example, we believe it is important to test whether a seasonal H1N1 strain or a rhinovirus would cause the same effects as influenza pH1N1. In addition, testing different aged mice, both older and younger, could unveil different observations providing further insight into the role of aging in these processes. For instance, newborn pups might react differently to the same virus or to different viruses (e.g., RSV) when compared to older animals (272).

\section{Role of $n A C h R s$}

Our data point to cholinergic signaling as mediating the effects of nicotine in our model. Specifically, our data point to $\alpha 7 \mathrm{nAChRs}$ in the processes investigated. Further investigations will be required to determined exactly how these receptors work. Also, there 
are many nAChRs (e.g., $\alpha 4 \beta 2, \alpha 3 \beta 4$ ) capable of mediating the effects of nicotine in lung and these should be considered for further study. Furthermore, the differences observed between our two different nicotine exposures with $\mathrm{pH} 1 \mathrm{~N} 1$ and between the genetic backgrounds, implicate specific timeframes where different nAChR may be acting. Implementing specific nAChR antagonists against these receptors and at different timepoints may begin to unravel the complexity of the processes discussed above.

\section{Immune Response Signaling and Implementing Immune Deficient Mice}

Immune signaling responses are likely involved in the functional and phenotypical changes described above (discussed briefly in Chapter I). The innate arm also plays a role in asthma development and hyperreactivity. Asthmatic patients are known to have increased production of Il-4, 5, and INF $\gamma$ in the sputum, compared to healthy controls (273). This cytokine response provides early support to drive the adaptive immune response (discussed above) (274). Murine models lacking NKT cells have been published to not develop AHR, $(275,276)$. Furthermore, NKT cells are increased in asthmatics following allergen challenge and may amplify the AHR (277-279).

The adaptive immune response plays a vital role in the pathogenesis of asthma AHR from extrinsic factors (such as ETS) signaling event cascade. $\mathrm{T}_{\mathrm{H}} 2$ and $\mathrm{T}_{\mathrm{H}} 17$ cells have been found to be increased in the lungs of human subjects with asthma and directly cause ARH $(274,280-282) . \mathrm{T}_{\mathrm{H}} 2$ cells arise from $\mathrm{CD}^{+}$cells stimulus mainly from innate lymphoid cell (ILC) and activated pulmonary epithelial cells (triggered by an external stimulus such as an allergen) $(274,283,284)$. This results in $\mathrm{T}_{\mathrm{H}} 2$ cell generation, 
subsequent recruitment of mast cells and basophils, $\operatorname{IgM}$ to $\operatorname{IgG} 1$ to $\operatorname{IgE}$ class switching (pulmonary B cells), and eosinophilia, which all contribute to AHR (274).

Whereas, Regulatory $\mathrm{T}$ (Treg) and $\gamma \delta \mathrm{T}$ cells are able to downregulate the inflammatory immune response in the lung and are thought to maintain immune homeostasis in the lung (274). Both of these cells are increased in the bronchoalveolar lavage fluid of asthmatics (274). Transfer of Treg cells has been published to inhibit the development of allergen-induced ARH and inflammation in the lung (285-289). Treg cells produce anti-inflammatory TGF $\beta$ as one method for inhibition of asthma ARH $(274,286)$. Treg cell production of TGF $\beta$ could cause an increase in extracellular matrix production which would promote the progression of OADs.

Epithelial cell immune signaling, in the setting of influenza infection, has been published to be able to induce AHR (through both $\operatorname{ILC}$ and $\mathrm{T}_{\mathrm{H}} 2$ cells) $(73,290)$. Interestingly, depletion of ILC (responsible for $\mathrm{T}_{\mathrm{H}} 2$ differentiation, discussed above) in an adult murine model of influenza results in loss of lung function by impairing airway remodeling and epithelial integrity (291). Upon treatment of mice with amphiregulin, which is produced from ILC, the disease state was reversed and mimicked immune efficient mice (291). Amphiregulin is a member of the epidermal growth factor family and implicated in a variety of physiological process including but not limited to regulation of lung morphogenesis (292). Lastly, in human sputum amphiregulin has been found to be associated with childhood asthma, which is not surprising knowing the implication of ILC on $\mathrm{T}_{\mathrm{H}} 2$ cell production (293).

Due to the evidence implicating the immune response it is important that future studies engage in further understanding these process in regard to OAD. Conducting 
microarray analysis of the lungs from 30 to 34-days of age (when we were seeing initial remodeling) could be implemented not only to evaluate tissue remodeling genes but also to determine differences of immune cytokine and chemokine signaling. Furthermore, quantitating innate and adaptive immune cells would also be a direction that would shed insight to the molecular pathogenesis driving the functional and phenotypical changes presented above. If differences in the immune response are discovered, from future studies, there are several immune deficient (knock out) animal models (RAG, SCID, NSG) that could be implemented to target the cells driving changes. In addition to this testing the effects of amphiregulin could also be an implemented approach as it has already been known to influence pulse oximetry of influenza infected animals (291). Other knock out models could be used as well such as SMAD knock out mice to see if TGF $\beta$ signaling is influencing the functional changes and collagen deposition around the airways. Furthermore, depending on the differences seen using different murine genetic backgrounds creating a double knock out including the $\alpha 7 \mathrm{nAChR}$ could provide evidence if cholinergic signaling is working in concert with the immune response to drive pulmonary functional abnormalities. 


\section{REFERENCES}

1. Duijts L. Fetal and infant origins of asthma. European journal of epidemiology 2012; 27: 5-14.

2. Papaiwannou A, Zarogoulidis P, Porpodis K, Spyratos D, Kioumis I, Pitsiou G, Pataka A, Tsakiridis K, Arikas S, Mpakas A, Tsiouda T, Katsikogiannis N, Kougioumtzi I, Machairiotis N, Siminelakis S, Kolettas A, Kessis G, Beleveslis T, Zarogoulidis $\mathrm{K}$. Asthma-chronic obstructive pulmonary disease overlap syndrome (ACOS): current literature review. Journal of thoracic disease 2014; 6 Suppl 1: S146-151.

3. Guerra S. Asthma and chronic obstructive pulmonary disease. Current opinion in allergy and clinical immunology 2009; 9: 409-416.

4. The Link between Asthma nd COPD. 2012 8-3-2015 [cited 2015 8-3-2015]. Available from: http://www.lung.org/associations/states/illinois/news/the-link-betweenasthma.html?referrer=https://www.google.com/.

5. Sly RM. Changing prevalence of allergic rhinitis and asthma. Annals of allergy, asthma \& immunology: official publication of the American College of Allergy, Asthma, \& Immunology 1999; 82: 233-248; quiz 248-252.

6. van Buul AR, Taube C. Treatment of severe asthma: entering the era of targeted therapy. Expert opinion on biological therapy 2015; 15: 1713-1725.

7. Global, regional, and national deaths, prevalence, disability-adjusted life years, and years lived with disability for chronic obstructive pulmonary disease and asthma, 1990-2015: a systematic analysis for the Global Burden of Disease Study 2015. The Lancet Respiratory medicine 2017; 5: 691-706.

8. European Resipiratory Society. European Lung White Book. http://www.erswhitebook.org/chapters/: Chapter 2: The economic burder of lung disease.

9. Nurmagambetov T, Kuwahara R, Garbe P. The Economic Burden of Asthma in the United States, 2008-2013. Annals of the American Thoracic Society 2018; 15: 348-356.

10. Guarascio AJ, Ray SM, Finch CK, Self TH. The clinical and economic burden of chronic obstructive pulmonary disease in the USA. ClinicoEconomics and outcomes research : CEOR 2013; 5: 235-245. 
11. Subbarao P, Mandhane PJ, Sears MR. Asthma: epidemiology, etiology and risk factors. CMAJ : Canadian Medical Association Journal 2009; 181: E181-190.

12. Bijanzadeh M, Mahesh PA, Ramachandra NB. An understanding of the genetic basis of asthma. The Indian Journal of Medical Research 2011; 134: 149-161.

13. Thomsen SF. Exploring the origins of asthma: Lessons from twin studies. European Clinical Respiratory Journal 2014; 1: 10.3402/ecrj.v3401.25535.

14. Cookson JB. Prevalence rates of asthma in developing countries and their comparison with those in Europe and North America. Chest 1987; 91: 97s-103s.

15. Cruz AA, Stelmach R, Ponte EV. Asthma prevalence and severity in low-resource communities. Current opinion in allergy and clinical immunology 2017; 17: 188193.

16. Walker B, Stokes LD, Warren R. Environmental factors associated with asthma. Journal of the National Medical Association 2003; 95: 152-166.

17. Duffy DL, Martin NG, Battistutta D, Hopper JL, Mathews JD. Genetics of asthma and hay fever in Australian twins. The American review of respiratory disease 1990; 142: 1351-1358.

18. Guo SW. Does higher concordance in monozygotic twins than in dizygotic twins suggest a genetic component? Human heredity 2001; 51: 121-132.

19. Laitinen T, Rasanen M, Kaprio J, Koskenvuo M, Laitinen LA. Importance of genetic factors in adolescent asthma: a population-based twin-family study. American journal of respiratory and critical care medicine 1998; 157: 1073-1078.

20. Huovinen E, Kaprio J, Laitinen LA, Koskenvuo M. Social predictors of adult asthma: a co-twin case-control study. Thorax 2001; 56: 234-236.

21. Nystad W, Roysamb E, Magnus P, Tambs K, Harris JR. A comparison of genetic and environmental variance structures for asthma, hay fever and eczema with symptoms of the same diseases: a study of Norwegian twins. International journal of epidemiology 2005; 34: 1302-1309.

22. Masoli M, Fabian D, Holt S, Beasley R. The global burden of asthma: executive summary of the GINA Dissemination Committee report. Allergy 2004; 59: 469478.

23. Liu AH SJ, Leung DYM. Nelson textbook of pediatrics. New Delhi: Elsevier; 2004.

24. Manuyakorn W, Howarth PH, Holgate ST. Airway remodelling in asthma and novel therapy. Asian Pacific journal of allergy and immunology 2013; 31: 3-10.

25. Burgess JK, Mauad T, Tjin G, Karlsson JC, Westergren-Thorsson G. The extracellular matrix - the under-recognized element in lung disease? The Journal of pathology 2016; 240: 397-409. 
26. Fahy JV, Corry DB, Boushey HA. Airway inflammation and remodeling in asthma. Current opinion in pulmonary medicine 2000; 6: 15-20.

27. Mauad T, Bel EH, Sterk PJ. Asthma therapy and airway remodeling. The Journal of allergy and clinical immunology 2007; 120: 997-1009; quiz 1010-1001.

28. Shahab L, Jarvis MJ, Britton J, West R. Prevalence, diagnosis and relation to tobacco dependence of chronic obstructive pulmonary disease in a nationally representative population sample. Thorax 2006; 61: 1043-1047.

29. Johannessen A, Bakke PS, Hardie JA, Eagan TM. Association of exposure to environmental tobacco smoke in childhood with chronic obstructive pulmonary disease and respiratory symptoms in adults. Respirology (Carlton, Vic) 2012; 17: 499-505.

30. Hwang SH, Hwang JH, Moon JS, Lee DH. Environmental tobacco smoke and children's health. Korean journal of pediatrics 2012; 55: 35-41.

31. Sharp RR, de Serres F, Newman L, Sandhaus RA, Walsh JW, Hood E, Harry GJ. Environmental, occupational, and genetic risk factors for alpha-1 antitrypsin deficiency. Environ Health Perspect 2003; 111: 1749-1752.

32. Stoller JK, Aboussouan LS. A review of alpha1-antitrypsin deficiency. American journal of respiratory and critical care medicine 2012; 185: 246-259.

33. Brode SK, Ling SC, Chapman KR. Alpha-1 antitrypsin deficiency: a commonly overlooked cause of lung disease. CMAJ : Canadian Medical Association Journal 2012; 184: 1365-1371.

34. Ramsey SD, Hobbs FD. Chronic obstructive pulmonary disease, risk factors, and outcome trials: comparisons with cardiovascular disease. Proceedings of the American Thoracic Society 2006; 3: 635-640.

35. Barnes PJ. Chronic obstructive pulmonary disease. The New England journal of medicine 2000; 343: 269-280.

36. Hoenderdos K, Condliffe A. The neutrophil in chronic obstructive pulmonary disease. American journal of respiratory cell and molecular biology 2013; 48: 531-539.

37. Chung KF. Cytokines in chronic obstructive pulmonary disease. The European respiratory journal Supplement 2001; 34: 50s-59s.

38. MacNee W. Pathogenesis of Chronic Obstructive Pulmonary Disease. Proceedings of the American Thoracic Society 2005; 2: 258-266.

39. Ignotz RA, Massague J. Transforming growth factor-beta stimulates the expression of fibronectin and collagen and their incorporation into the extracellular matrix. The Journal of biological chemistry 1986; 261: 4337-4345. 
40. Biernacka A, Dobaczewski M, Frangogiannis NG. TGF- $\beta$ signaling in fibrosis. Growth factors (Chur, Switzerland) 2011; 29: 196-202.

41. Lappalainen U, Whitsett JA, Wert SE, Tichelaar JW, Bry K. Interleukin-1beta causes pulmonary inflammation, emphysema, and airway remodeling in the adult murine lung. American journal of respiratory cell and molecular biology 2005; 32: 311318.

42. Wongtrakool C, Wang N, Hyde DM, Roman J, Spindel ER. Prenatal nicotine exposure alters lung function and airway geometry through alpha7 nicotinic receptors. American journal of respiratory cell and molecular biology 2012; 46: 695-702.

43. Stern DA, Morgan WJ, Wright AL, Guerra S, Martinez FD. Poor airway function in early infancy and lung function by age 22 years: a non-selective longitudinal cohort study. Lancet (London, England) 2007; 370: 758-764.

44. Lange P, Celli B, Agusti A, Boje Jensen G, Divo M, Faner R, Guerra S, Marott JL, Martinez FD, Martinez-Camblor P, Meek P, Owen CA, Petersen H, Pinto-Plata V, Schnohr P, Sood A, Soriano JB, Tesfaigzi Y, Vestbo J. Lung-Function Trajectories Leading to Chronic Obstructive Pulmonary Disease. The New England journal of medicine 2015; 373: 111-122.

45. Prevention CfDCa. Asthma. 2016 February 1, 2016 Febuary 15, 2016]. Available from: http://www.cdc.gov/asthma/.

46. Sly PD. The early origins of asthma: who is really at risk? Current opinion in allergy and clinical immunology 2011; 11: 24-28.

47. Cunningham J, Dockery DW, Speizer FE. Maternal smoking during pregnancy as a predictor of lung function in children. American journal of epidemiology 1994; 139: 1139-1152.

48. Spindel ER, McEvoy CT. The Role of Nicotine in the Effects of Maternal Smoking during Pregnancy on Lung Development and Childhood Respiratory Disease. Implications for Dangers of E-Cigarettes. American journal of respiratory and critical care medicine 2016; 193: 486-494.

49. Hayatbakhsh MR, Sadasivam S, Mamun AA, Najman JM, Williams GM, O'Callaghan MJ. Maternal smoking during and after pregnancy and lung function in early adulthood: a prospective study. Thorax 2009; 64: 810-814.

50. Stocks J, Hislop A, Sonnappa S. Early lung development: lifelong effect on respiratory health and disease. The Lancet Respiratory medicine 2013; 1: 728742.

51. Ivorra C, Garcia-Vicent C, Ponce F, Ortega-Evangelio G, Fernandez-Formoso JA, Lurbe E. High cotinine levels are persistent during the first days of life in 
newborn second hand smokers. Drug and alcohol dependence 2014; 134: 275 279.

52. Chazeron I, Daval S, Ughetto S, Richard D, Nicolay A, Lemery D, Llorca PM, Coudore F. GC-MS determined cotinine in an epidemiological study on smoking status at delivery. Pulmonary pharmacology \& therapeutics 2008; 21: 485-488.

53. Drake P, Driscoll AK, Mathews TJ. Cigarette Smoking During Pregnancy: United States, 2016. NCHS data brief 2018: 1-8.

54. Gibbs K, Collaco JM, McGrath-Morrow SA. Impact of Tobacco Smoke and Nicotine Exposure on Lung Development. Chest 2016; 149: 552-561.

55. Cook DG, Strachan DP, Carey IM. Health effects of passive smoking. 9. Parental smoking and spirometric indices in children. Thorax 1998; 53: 884-893.

56. Milcarz K, Bak-Romaniszyn L, Kaleta D. Environmental Tobacco Smoke Exposure and Smoke-Free Rules in Homes among Socially-Disadvantaged Populations in Poland. International Journal of Environmental Research and Public Health 2017; 14: 447.

57. Ross EJ, Graham DL, Money KM, Stanwood GD. Developmental consequences of fetal exposure to drugs: what we know and what we still must learn. Neuropsychopharmacology : official publication of the American College of Neuropsychopharmacology 2015; 40: 61-87.

58. Lindstrom J. Nicotinic acetylcholine receptors in health and disease. Molecular neurobiology 1997; 15: 193-222.

59. Albuquerque EX, Pereira EF, Alkondon M, Rogers SW. Mammalian nicotinic acetylcholine receptors: from structure to function. Physiological reviews 2009; 89: 73-120.

60. Gotti C, Clementi F. Neuronal nicotinic receptors: from structure to pathology. Progress in neurobiology 2004; 74: 363-396.

61. Lambers DS, Clark KE. The maternal and fetal physiologic effects of nicotine. Seminars in perinatology 1996; 20: 115-126.

62. Sekhon HS, Jia Y, Raab R, Kuryatov A, Pankow JF, Whitsett JA, Lindstrom J, Spindel ER. Prenatal nicotine increases pulmonary alpha7 nicotinic receptor expression and alters fetal lung development in monkeys. The Journal of clinical investigation 1999; 103: 637-647.

63. Sekhon HS, Keller JA, Benowitz NL, Spindel ER. Prenatal nicotine exposure alters pulmonary function in newborn rhesus monkeys. American journal of respiratory and critical care medicine 2001; 164: 989-994.

64. Wongtrakool C, Roser-Page S, Rivera HN, Roman J. Nicotine alters lung branching morphogenesis through the alpha7 nicotinic acetylcholine receptor. American 
journal of physiology Lung cellular and molecular physiology 2007; 293: L611618.

65. Girolamo Pelaia AV, Luca Gallelli,Teresa Renda, Mario Cazzola, Rosario Maselli, Serafino A. Marsico. Respiratory infections and asthma. Respiratory Medicine 2006; 100: 775-784.

66. Teichtahl H, Buckmaster N, Pertnikovs E. The incidence of respiratory tract infection in adults requiring hospitalization for asthma. Chest 1997; 112: 591-596.

67. Gavala ML, Bertics PJ, Gern JE. Rhinoviruses, allergic inflammation, and asthma. Immunological reviews 2011; 242: 69-90.

68. Tregoning JS, Schwarze J. Respiratory viral infections in infants: causes, clinical symptoms, virology, and immunology. Clinical microbiology reviews 2010; 23: 74-98.

69. Kusel MM, de Klerk NH, Kebadze T, Vohma V, Holt PG, Johnston SL, Sly PD. Early-life respiratory viral infections, atopic sensitization, and risk of subsequent development of persistent asthma. The Journal of allergy and clinical immunology 2007; 119: 1105-1110.

70. Jackson DJ, Gangnon RE, Evans MD, Roberg KA, Anderson EL, Pappas TE, Printz MC, Lee WM, Shult PA, Reisdorf E, Carlson-Dakes KT, Salazar LP, DaSilva DF, Tisler CJ, Gern JE, Lemanske RF, Jr. Wheezing rhinovirus illnesses in early life predict asthma development in high-risk children. American journal of respiratory and critical care medicine 2008; 178: 667-672.

71. Dulek DE, Peebles RS, Jr. Viruses and asthma. Biochimica et biophysica acta 2011; 1810: 1080-1090.

72. You D, Ripple M, Balakrishna S, Troxclair D, Sandquist D, Ding L, Ahlert TA, Cormier SA. Inchoate CD8+ T cell responses in neonatal mice permit influenzainduced persistent pulmonary dysfunction. Journal of immunology (Baltimore, Md : 1950) 2008; 181: 3486-3494.

73. Chang YJ, Kim HY, Albacker LA, Baumgarth N, McKenzie AN, Smith DE, Dekruyff RH, Umetsu DT. Innate lymphoid cells mediate influenza-induced airway hyper-reactivity independently of adaptive immunity. Nature immunology 2011; 12: 631-638.

74. Braciale TJ, Sun J, Kim TS. Regulating the adaptive immune response to respiratory virus infection. Nature reviews Immunology 2012; 12: 295-305.

75. Kreijtz JH, Fouchier RA, Rimmelzwaan GF. Immune responses to influenza virus infection. Virus research 2011; 162: 19-30.

76. Iwasaki A, Pillai PS. Innate immunity to influenza virus infection. Nature reviews Immunology 2014; 14: 315-328. 
77. Oslund KL, Baumgarth N. Influenza-induced innate immunity: regulators of viral replication, respiratory tract pathology \& adaptive immunity. Future virology 2011; 6: 951-962.

78. Schultz-Cherry S, Hinshaw VS. Influenza virus neuraminidase activates latent transforming growth factor beta. Journal of virology 1996; 70: 8624-8629.

79. Gazit R, Gruda R, Elboim M, Arnon TI, Katz G, Achdout H, Hanna J, Qimron U, Landau G, Greenbaum E, Zakay-Rones Z, Porgador A, Mandelboim O. Lethal influenza infection in the absence of the natural killer cell receptor gene Ncr1. Nature immunology 2006; 7: 517-523.

80. Chen K, Kolls JK. T Cell-Mediated Host Immune Defenses in the Lung. Annual review of immunology 2013; 31: 605-633.

81. Denney L, Branchett W, Gregory LG, Oliver RA, Lloyd CM. Epithelial-derived TGF-beta1 acts as a pro-viral factor in the lung during influenza $\mathrm{A}$ infection. Mucosal immunology 2017.

82. Williams AE, Humphreys IR, Cornere M, Edwards L, Rae A, Hussell T. TGF-beta prevents eosinophilic lung disease but impairs pathogen clearance. Microbes and infection / Institut Pasteur 2005; 7: 365-374.

83. D'Alessio FR, Tsushima K, Aggarwal NR, West EE, Willett MH, Britos MF, Pipeling MR, Brower RG, Tuder RM, McDyer JF, King LS. CD4+CD25+Foxp3+ Tregs resolve experimental lung injury in mice and are present in humans with acute lung injury. The Journal of clinical investigation 2009; 119: 2898-2913.

84. Stephens MB, Yew KS. Diagnosis of chronic obstructive pulmonary disease. American family physician 2008; 78: 87-92.

85. Webb WR. Radiology of obstructive pulmonary disease. AJR American journal of roentgenology 1997; 169: 637-647.

86. Lynch DA, Austin JH, Hogg JC, Grenier PA, Kauczor HU, Bankier AA, Barr RG, Colby TV, Galvin JR, Gevenois PA, Coxson HO, Hoffman EA, Newell JD, Jr., Pistolesi M, Silverman EK, Crapo JD. CT-Definable Subtypes of Chronic Obstructive Pulmonary Disease: A Statement of the Fleischner Society. Radiology 2015; 277: 192-205.

87. Richards JC, Lynch D, Koelsch T, Dyer D. Imaging of Asthma. Immunology and allergy clinics of North America 2016; 36: 529-545.

88. Parker MJ. Interpreting spirometry: the basics. Otolaryngologic clinics of North America 2014; 47: 39-53.

89. Barreiro TJ, Perillo I. An approach to interpreting spirometry. American family physician 2004; 69: 1107-1114. 
90. Pierce R. Spirometry: an essential clinical measurement. Australian family physician 2005; 34: 535-539.

91. Miller MR, Hankinson J, Brusasco V, Burgos F, Casaburi R, Coates A, Crapo R, Enright P, van der Grinten CP, Gustafsson P, Jensen R, Johnson DC, MacIntyre N, McKay R, Navajas D, Pedersen OF, Pellegrino R, Viegi G, Wanger J.

Standardisation of spirometry. The European respiratory journal 2005; 26: 319 338.

92. Quanjer PH, Tammeling GJ, Cotes JE, Pedersen OF, Peslin R, Yernault JC. [Lung volumes and forced ventilatory flows. Work Group on Standardization of Respiratory Function Tests. European Community for Coal and Steel. Official position of the European Respiratory Society]. Revue des maladies respiratoires 1994; 11 Suppl 3: 5-40.

93. Wanger J, Clausen JL, Coates A, Pedersen OF, Brusasco V, Burgos F, Casaburi R, Crapo R, Enright P, van der Grinten CP, Gustafsson P, Hankinson J, Jensen R, Johnson D, Macintyre N, McKay R, Miller MR, Navajas D, Pellegrino R, Viegi G. Standardisation of the measurement of lung volumes. The European respiratory journal 2005; 26: 511-522.

94. Alhassan S, Hattab Y, Bajwa O, Bihler E, Singh AC. Asthma. Critical care nursing quarterly 2016; 39: 110-123.

95. Pike KC, Levy ML, Moreiras J, Fleming L. Managing problematic severe asthma: beyond the guidelines. Archives of disease in childhood 2017.

96. Cunnington D, Smith N, Steed K, Rosengarten P, Kelly AM, Teichtahl H. Oral versus intravenous corticosteroids in adults hospitalised with acute asthma. Pulmonary pharmacology \& therapeutics 2005; 18: 207-212.

97. Rowe BH, Bretzlaff JA, Bourdon C, Bota GW, Camargo CA, Jr. Magnesium sulfate for treating exacerbations of acute asthma in the emergency department. The Cochrane database of systematic reviews 2000: Cd001490.

98. Townley RG, Suliaman F. The mechanism of corticosteroids in treating asthma. Annals of allergy 1987; 58: 1-6.

99. Fanta CH. Asthma. The New England journal of medicine 2009; 360: 1002-1014.

100. Juniper EF, Kline PA, Vanzieleghem MA, Ramsdale EH, O'Byrne PM, Hargreave FE. Effect of long-term treatment with an inhaled corticosteroid (budesonide) on airway hyperresponsiveness and clinical asthma in nonsteroid-dependent asthmatics. The American review of respiratory disease 1990; 142: 832-836.

101. Sin DD, Man J, Sharpe H, Gan WQ, Man SF. Pharmacological management to reduce exacerbations in adults with asthma: a systematic review and metaanalysis. Jama 2004; 292: 367-376. 
102. Donahue JG, Weiss ST, Livingston JM, Goetsch MA, Greineder DK, Platt R. Inhaled steroids and the risk of hospitalization for asthma. Jama 1997; 277: 887891.

103. Suissa S, Ernst P, Benayoun S, Baltzan M, Cai B. Low-dose inhaled corticosteroids and the prevention of death from asthma. The New England journal of medicine 2000; 343: 332-336.

104. Barnes PJ. Inhaled Corticosteroids. Pharmaceuticals 2010; 3: 514-540.

105. Barnes NC, Miller CJ. Effect of leukotriene receptor antagonist therapy on the risk of asthma exacerbations in patients with mild to moderate asthma: an integrated analysis of zafirlukast trials. Thorax 2000; 55: 478-483.

106. Expert Panel Report 3 (EPR-3): Guidelines for the Diagnosis and Management of Asthma-Summary Report 2007. The Journal of allergy and clinical immunology 2007; 120: S94-138.

107. Humbert M, Beasley R, Ayres J, Slavin R, Hebert J, Bousquet J, Beeh KM, Ramos S, Canonica GW, Hedgecock S, Fox H, Blogg M, Surrey K. Benefits of omalizumab as add-on therapy in patients with severe persistent asthma who are inadequately controlled despite best available therapy (GINA 2002 step 4 treatment): INNOVATE. Allergy 2005; 60: 309-316.

108. Balkissoon R, Lommatzsch S, Carolan B, Make B. Chronic obstructive pulmonary disease: a concise review. The Medical clinics of North America 2011; 95: 1125 1141 .

109. Rice KL, Dewan N, Bloomfield HE, Grill J, Schult TM, Nelson DB, Kumari S, Thomas M, Geist LJ, Beaner C, Caldwell M, Niewoehner DE. Disease management program for chronic obstructive pulmonary disease: a randomized controlled trial. American journal of respiratory and critical care medicine 2010; 182: 890-896.

110. Aun MV, Bonamichi-Santos R, Arantes-Costa FM, Kalil J, Giavina-Bianchi P. Animal models of asthma: utility and limitations. Journal of Asthma and Allergy 2017; 10: 293-301.

111. Ghorani V, Boskabady MH, Khazdair MR, Kianmeher M. Experimental animal models for COPD: a methodological review. Tobacco induced diseases 2017; 15: 25.

112. Suki B, Bartolak-Suki E, Rocco PRM. Elastase-Induced Lung Emphysema Models in Mice. Methods in molecular biology (Clifton, NJ) 2017; 1639: 67-75.

113. Wright JL, Cosio M, Churg A. Animal models of chronic obstructive pulmonary disease. American journal of physiology Lung cellular and molecular physiology 2008; 295: L1-15. 
114. Devos FC, Maaske A, Robichaud A, Pollaris L, Seys S, Lopez CA, Verbeken E, Tenbusch M, Lories R, Nemery B, Hoet PH, Vanoirbeek JA. Forced expiration measurements in mouse models of obstructive and restrictive lung diseases. Respiratory research 2017; 18: 123.

115. Leberl M, Kratzer A, Taraseviciene-Stewart L. Tobacco smoke induced COPD/emphysema in the animal model-are we all on the same page? Frontiers in physiology 2013; 4: 91.

116. Techniques \& Measurements. [cited 2018 April]. Available from: http://www.scireq.com/flexivent/techniques-and-measurements.

117. Schulz H, Johner C, Eder G, Ziesenis A, Reitmeier P, Heyder J, Balling R. Respiratory mechanics in mice: strain and sex specific differences. Acta physiologica Scandinavica 2002; 174: 367-375.

118. Chang HY, Mitzner W, Watson J. Variation in airway responsiveness of male C57BL/6 mice from 5 vendors. Journal of the American Association for Laboratory Animal Science : JAALAS 2012; 51: 401-406.

119. McGovern TK, Robichaud A, Fereydoonzad L, Schuessler TF, Martin JG. Evaluation of respiratory system mechanics in mice using the forced oscillation technique. Journal of visualized experiments : JoVE 2013: e50172.

120. Shalaby KH, Gold LG, Schuessler TF, Martin JG, Robichaud A. Combined forced oscillation and forced expiration measurements in mice for the assessment of airway hyperresponsiveness. Respiratory research 2010; 11: 82.

121. Siddiqui S, Tsuchiya K, Risse PA, Bullimore SR, Benedetti A, Martin JG. Site of allergic airway narrowing and the influence of exogenous surfactant in the Brown Norway rat. PloS one 2012; 7: e29381.

122. Ford ES, Murphy LB, Khavjou O, Giles WH, Holt JB, Croft JB. Total and statespecific medical and absenteeism costs of COPD among adults aged $>/=18$ years in the United States for 2010 and projections through 2020. Chest 2015; 147: 3145.

123. Bloom B, Jones LI, Freeman G. Summary health statistics for U.S. children: National Health Interview Survey, 2012. Vital and health statistics Series 10, Data from the National Health Survey 2013: 1-81.

124. The Health Consequences of Involuntary Exposure to Tobacco Smoke: A Report of the Surgeon General. U.S. Department of Health and Human Services, 2006.

125. Tong VT, Dietz PM, Morrow B, D'Angelo DV, Farr SL, Rockhill KM, England LJ. Trends in smoking before, during, and after pregnancy--Pregnancy Risk Assessment Monitoring System, United States, 40 sites, 2000-2010. Morbidity and mortality weekly report Surveillance summaries (Washington, DC : 2002) 2013; 62: 1-19. 
126. Hanrahan JP, Tager IB, Segal MR, Tosteson TD, Castile RG, Van Vunakis H, Weiss ST, Speizer FE. The effect of maternal smoking during pregnancy on early infant lung function. The American review of respiratory disease 1992; 145: 1129-1135.

127. Wickstrom R. Effects of nicotine during pregnancy: human and experimental evidence. Current neuropharmacology 2007; 5: 213-222.

128. Coleman T, Britton J, Thornton J. Nicotine replacement therapy in pregnancy. BMJ (Clinical research ed) 2004; 328: 965-966.

129. Nelson EA, Taylor BJ. International Child Care Practices Study: infant sleep position and parental smoking. Early human development 2001; 64: 7-20.

130. Cohen RT, Raby BA, Van Steen K, Fuhlbrigge AL, Celedon JC, Rosner BA, Strunk $\mathrm{RC}$, Zeiger RS, Weiss ST. In utero smoke exposure and impaired response to inhaled corticosteroids in children with asthma. The Journal of allergy and clinical immunology 2010; 126: 491-497.

131. Tager IB, Hanrahan JP, Tosteson TD, Castile RG, Brown RW, Weiss ST, Speizer FE. Lung function, pre- and post-natal smoke exposure, and wheezing in the first year of life. The American review of respiratory disease 1993; 147: 811-817.

132. Stick SM, Burton PR, Gurrin L, Sly PD, LeSouef PN. Effects of maternal smoking during pregnancy and a family history of asthma on respiratory function in newborn infants. Lancet (London, England) 1996; 348: 1060-1064.

133. Hipple B, Lando H, Klein J, Winickoff J. Global teens and tobacco: a review of the globalization of the tobacco epidemic. Current problems in pediatric and adolescent health care 2011; 41:216-230.

134. Sekhon HS, Keller JA, Proskocil BJ, Martin EL, Spindel ER. Maternal nicotine exposure upregulates collagen gene expression in fetal monkey lung. Association with alpha7 nicotinic acetylcholine receptors. American journal of respiratory cell and molecular biology 2002; 26: 31-41.

135. Liu J, Naeem E, Tian J, Lombardi V, Kwong K, Akbari O, Torday JS, Rehan VK. Sex-specific perinatal nicotine-induced asthma in rat offspring. American journal of respiratory cell and molecular biology 2013; 48: 53-62.

136. Rowell PP, Hurst HE, Marlowe C, Bennett BD. Oral administration of nicotine: its uptake and distribution after chronic administration to mice. Journal of pharmacological methods 1983; 9: 249-261.

137. Hoyle GW, Chang W, Chen J, Schlueter CF, Rando RJ. Deviations from Haber's Law for multiple measures of acute lung injury in chlorine-exposed mice. Toxicological sciences : an official journal of the Society of Toxicology 2010; 118: 696-703. 
138. Chang W, Chen J, Schlueter CF, Rando RJ, Pathak YV, Hoyle GW. Inhibition of chlorine-induced lung injury by the type 4 phosphodiesterase inhibitor rolipram. Toxicology and applied pharmacology 2012; 263: 251-258.

139. Hadi AM, Mouchaers KT, Schalij I, Grunberg K, Meijer GA, Vonk-Noordegraaf A, van der Laarse WJ, Belien JA. Rapid quantification of myocardial fibrosis: a new macro-based automated analysis. Cellular oncology (Dordrecht) 2011; 34: 343354.

140. Song N, Liu J, Shaheen S, Du L, Proctor M, Roman J, Yu J. Vagotomy attenuates bleomycin-induced pulmonary fibrosis in mice. Scientific reports 2015; 5: 13419.

141. Hsia CC, Hyde DM, Ochs M, Weibel ER. An official research policy statement of the American Thoracic Society/European Respiratory Society: standards for quantitative assessment of lung structure. American journal of respiratory and critical care medicine 2010; 181: 394-418.

142. Dunnill MS. Quantitative methods in the study of pulmonary pathology. Thorax 1962; 17: 320-328.

143. Walker GA, Shostak J. Common Statistical Methods for Clinical Research with SAS ${ }^{\circledR}$ Examples 2nd Edition. Cary, NC: SAS Institute Inc; 2010.

144. The SAS System V9. Cary, NC: SAS Institute Inc.; 2003.

145. Busse WW, Banks-Schlegel SP, Larsen GL. Effects of growth and development on lung function. Models for study of childhood asthma. American journal of respiratory and critical care medicine 1997; 156: 314-319.

146. Miller MD, Marty MA. Impact of environmental chemicals on lung development. Environ Health Perspect 2010; 118: 1155-1164.

147. Rehan VK, Liu J, Sakurai R, Torday JS. Perinatal nicotine-induced transgenerational asthma. American journal of physiology Lung cellular and molecular physiology 2013; 305: L501-507.

148. Neuman A, Hohmann C, Orsini N, Pershagen G, Eller E, Kjaer HF, Gehring U, Granell R, Henderson J, Heinrich J, Lau S, Nieuwenhuijsen M, Sunyer J, Tischer C, Torrent M, Wahn U, Wijga AH, Wickman M, Keil T, Bergstrom A. Maternal smoking in pregnancy and asthma in preschool children: a pooled analysis of eight birth cohorts. American journal of respiratory and critical care medicine 2012; 186: 1037-1043.

149. Lannero E, Wickman M, Pershagen G, Nordvall L. Maternal smoking during pregnancy increases the risk of recurrent wheezing during the first years of life (BAMSE). Respiratory research 2006; 7: 3.

150. Hoo AF, Henschen M, Dezateux C, Costeloe K, Stocks J. Respiratory function among preterm infants whose mothers smoked during pregnancy. American journal of respiratory and critical care medicine 1998; 158: 700-705. 
151. McEvoy CT, Schilling D, Clay N, Jackson K, Go MD, Spitale P, Bunten C, Leiva M, Gonzales D, Hollister-Smith J, Durand M, Frei B, Buist AS, Peters D, Morris $\mathrm{CD}$, Spindel ER. Vitamin C supplementation for pregnant smoking women and pulmonary function in their newborn infants: a randomized clinical trial. Jama 2014; 311: 2074-2082.

152. Hu LW, Yang M, Chen S, Shah K, Hailegiorgis Y, Burgens R, Vaughn M, Huang J, Xaverius P, Paul G, Morawska L, Lu T, Lin S, Zhong SQ, Kong ML, Xie YQ, Hao YT, Zeng XW, Qian Z, Dong GH. Effects of in utero and Postnatal Exposure to Secondhand Smoke on Lung Function by Gender and Asthma Status: The Seven Northeastern Cities (SNEC) Study. Respiration; international review of thoracic diseases 2017; 93: 189-197.

153. Liu J, Sakurai R, O'Roark EM, Kenyon NJ, Torday JS, Rehan VK. PPARgamma agonist rosiglitazone prevents perinatal nicotine exposure-induced asthma in rat offspring. American journal of physiology Lung cellular and molecular physiology 2011; 300: L710-717.

154. Magnus MC, Haberg SE, Karlstad O, Nafstad P, London SJ, Nystad W. Grandmother's smoking when pregnant with the mother and asthma in the grandchild: the Norwegian Mother and Child Cohort Study. Thorax 2015; 70: 237-243.

155. Sasaki J. [Compounds in tobacco smoke and pathogenesis of the diseases]. Nihon rinsho Japanese journal of clinical medicine 2013; 71: 383-389.

156. Schittny JC. Development of the lung. Cell and Tissue Research 2017; 367: 427444.

157. Warburton D, El-Hashash A, Carraro G, Tiozzo C, Sala F, Rogers O, De Langhe S, Kemp PJ, Riccardi D, Torday J, Bellusci S, Shi W, Lubkin SR, Jesudason E. Lung Organogenesis. Current Topics in Developmental Biology 2010; 90: 73-158.

158. Rehan VK, Asotra K, Torday JS. The effects of smoking on the developing lung: insights from a biologic model for lung development, homeostasis, and repair. Lung 2009; 187: 281-289.

159. Sandberg KL, Pinkerton KE, Poole SD, Minton PA, Sundell HW. Fetal nicotine exposure increases airway responsiveness and alters airway wall composition in young lambs. Respiratory physiology \& neurobiology 2011; 176: 57-67.

160. Maritz GS, Woolward K. Effect of maternal nicotine exposure on neonatal lung elastic tissue and possible consequences. South African medical journal $=$ SuidAfrikaanse tydskrif vir geneeskunde 1992; 81: 517-519.

161. Conklin BS, Zhao W, Zhong DS, Chen C. Nicotine and cotinine up-regulate vascular endothelial growth factor expression in endothelial cells. The American journal of pathology 2002; 160: 413-418. 
162. Joss-Moore LA, Albertine KH, Lane RH. Epigenetics and the developmental origins of lung disease. Molecular genetics and metabolism 2011; 104: 61-66.

163. Gong M, Liu J, Sakurai R, Corre A, Anthony S, Rehan VK. Perinatal nicotine exposure suppresses PPARgamma epigenetically in lung alveolar interstitial fibroblasts. Molecular genetics and metabolism 2015; 114: 604-612.

164. Hylkema MN, Blacquiere MJ. Intrauterine effects of maternal smoking on sensitization, asthma, and chronic obstructive pulmonary disease. Proceedings of the American Thoracic Society 2009; 6: 660-662.

165. Mizikova I, Ruiz-Camp J, Steenbock H, Madurga A, Vadasz I, Herold S, Mayer K, Seeger W, Brinckmann J, Morty RE. Collagen and elastin cross-linking is altered during aberrant late lung development associated with hyperoxia. American journal of physiology Lung cellular and molecular physiology 2015; 308: L11451158 .

166. Jalali M, Nikravesh MR, Moeen AA, Mohammadi S, Karimfar MH. Effects of Maternal Nicotine Exposure on Expression of Collagen Type IV and its Roles on Pulmonary Bronchogenesis and Alveolarization in Newborn Mice. Iranian journal of allergy, asthma, and immunology 2010; 9: 169-173.

167. Papandrinopoulou D, Tzouda V, Tsoukalas G. Lung compliance and chronic obstructive pulmonary disease. Pulmonary medicine 2012; 2012: 542769.

168. Noel A, Xiao R, Perveen Z, Zaman H, Le Donne V, Penn A. Sex-specific lung functional changes in adult mice exposed only to second-hand smoke in utero. Respiratory research 2017; 18: 104.

169. Cormier SA, You D, Honnegowda S. The use of a neonatal mouse model to study respiratory syncytial virus infections. Expert review of anti-infective therapy 2010; 8: 1371-1380.

170. You D, Siefker DT, Shrestha B, Saravia J, Cormier SA. Building a better neonatal mouse model to understand infant respiratory syncytial virus disease. Respiratory research 2015; 16: 91.

171. Bouvier NM, Lowen AC. Animal Models for Influenza Virus Pathogenesis and Transmission. Viruses 2010; 2: 1530-1563.

172. Bem RA, Domachowske JB, Rosenberg HF. Animal models of human respiratory syncytial virus disease. American journal of physiology Lung cellular and molecular physiology 2011; 301: L148-156.

173. Lines JL, Hoskins S, Hollifield M, Cauley LS, Garvy BA. The migration of T cells in response to influenza virus is altered in neonatal mice. Journal of immunology (Baltimore, Md : 1950) 2010; 185: 2980-2988. 
174. Bonnelykke K, Vissing NH, Sevelsted A, Johnston SL, Bisgaard H. Association between respiratory infections in early life and later asthma is independent of virus type. The Journal of allergy and clinical immunology 2015; 136: 81-86.e84.

175. You D. Neonatal Respiratory Virus Infection Shapes Pulmonary Function in Adult Mice LSU Doctoral Dissertations: Louisiana State University 2008.

176. Zhou H, Thompson WW, Viboud CG, Ringholz CM, Cheng P-Y, Steiner C, Abedi GR, Anderson LJ, Brammer L, Shay DK. Hospitalizations Associated With Influenza and Respiratory Syncytial Virus in the United States, 1993-2008. Clinical Infectious Diseases: An Official Publication of the Infectious Diseases Society of America 2012; 54: 1427-1436.

177. Matias G, Taylor R, Haguinet F, Schuck-Paim C, Lustig R, Shinde V. Estimates of hospitalization attributable to influenza and RSV in the US during 1997-2009, by age and risk status. BMC public health 2017; 17: 271.

178. You D, Becnel D, Wang K, Ripple M, Daly M, Cormier SA. Exposure of neonates to respiratory syncytial virus is critical in determining subsequent airway response in adults. Respiratory research 2006; 7: 107.

179. Morens DM, Taubenberger JK, Harvey HA, Memoli MJ. The 1918 influenza pandemic: lessons for 2009 and the future. Critical care medicine 2010; 38: e1020.

180. Garten RJ, Davis CT, Russell CA, Shu B, Lindstrom S, Balish A, Sessions WM, Xu X, Skepner E, Deyde V, Okomo-Adhiambo M, Gubareva L, Barnes J, Smith CB, Emery SL, Hillman MJ, Rivailler P, Smagala J, de Graaf M, Burke DF, Fouchier RA, Pappas C, Alpuche-Aranda CM, Lopez-Gatell H, Olivera H, Lopez I, Myers CA, Faix D, Blair PJ, Yu C, Keene KM, Dotson PD, Jr., Boxrud D, Sambol AR, Abid SH, St George K, Bannerman T, Moore AL, Stringer DJ, Blevins P, Demmler-Harrison GJ, Ginsberg M, Kriner P, Waterman S, Smole S, Guevara HF, Belongia EA, Clark PA, Beatrice ST, Donis R, Katz J, Finelli L, Bridges CB, Shaw M, Jernigan DB, Uyeki TM, Smith DJ, Klimov AI, Cox NJ. Antigenic and genetic characteristics of swine-origin $2009 \mathrm{~A}(\mathrm{H} 1 \mathrm{~N} 1)$ influenza viruses circulating in humans. Science (New York, NY) 2009; 325: 197-201.

181. Karageorgopoulos DE, Vouloumanou EK, Korbila IP, Kapaskelis A, Falagas ME. Age distribution of cases of 2009 (H1N1) pandemic influenza in comparison with seasonal influenza. PloS one 2011; 6: e21690.

182. Hancock K, Veguilla V, Lu X, Zhong W, Butler EN, Sun H, Liu F, Dong L, DeVos JR, Gargiullo PM, Brammer TL, Cox NJ, Tumpey TM, Katz JM. Cross-reactive antibody responses to the 2009 pandemic H1N1 influenza virus. The New England journal of medicine 2009; 361: 1945-1952.

183. Srivastava B, Blazejewska P, Hessmann M, Bruder D, Geffers R, Mauel S, Gruber $\mathrm{AD}$, Schughart K. Host genetic background strongly influences the response to influenza a virus infections. PloS one 2009; 4: e4857. 
184. Sun J, Madan R, Karp CL, Braciale TJ. Effector T cells control lung inflammation during acute influenza virus infection by producing IL-10. Nature medicine 2009; 15: $277-284$.

185. Ilyushina NA, Khalenkov AM, Seiler JP, Forrest HL, Bovin NV, Marjuki H, Barman S, Webster RG, Webby RJ. Adaptation of pandemic H1N1 influenza viruses in mice. Journal of virology 2010; 84: 8607-8616.

186. Manicassamy B, Medina RA, Hai R, Tsibane T, Stertz S, Nistal-Villan E, Palese P, Basler CF, Garcia-Sastre A. Protection of mice against lethal challenge with 2009 H1N1 influenza A virus by 1918-like and classical swine H1N1 based vaccines. PLoS pathogens 2010; 6: e1000745.

187. Le Goffic R, Arshad MI, Rauch M, L'Helgoualc'h A, Delmas B, Piquet-Pellorce C, Samson M. Infection with influenza virus induces IL-33 in murine lungs. American journal of respiratory cell and molecular biology 2011; 45: 1125-1132.

188. Trammell RA, Liberati TA, Toth LA. Host genetic background and the innate inflammatory response of lung to influenza virus. Microbes and infection / Institut Pasteur 2012; 14: 50-58.

189. Lanzer KG, Johnson LL, Woodland DL, Blackman MA. Impact of ageing on the response and repertoire of influenza virus-specific CD4 T cells. Immunity \& Ageing : I \& A 2014; 11: 9-9.

190. Bender BS, Taylor SF, Zander DS, Cottey R. Pulmonary immune response of young and aged mice after influenza challenge. The Journal of laboratory and clinical medicine 1995; 126: 169-177.

191. Simon AK, Hollander GA, McMichael A. Evolution of the immune system in humans from infancy to old age. Proceedings Biological sciences 2015; 282: 20143085 .

192. Mestas J, Hughes CC. Of mice and not men: differences between mouse and human immunology. Journal of immunology (Baltimore, Md : 1950) 2004; 172: 27312738 .

193. Camp JV, Chu YK, Chung DH, McAllister RC, Adcock RS, Gerlach RL, Wiemken TL, Peyrani P, Ramirez JA, Summersgill JT, Jonsson CB. Phenotypic differences in virulence and immune response in closely related clinical isolates of influenza A 2009 H1N1 pandemic viruses in mice. PloS one 2013; 8: e56602.

194. Szretter KJ, Balish AL, Katz JM. Influenza: propagation, quantification, and storage. Current protocols in microbiology 2006; Chapter 15: Unit 15G.11.

195. Langford DJ, Bailey AL, Chanda ML, Clarke SE, Drummond TE, Echols S, Glick S, Ingrao J, Klassen-Ross T, Lacroix-Fralish ML, Matsumiya L, Sorge RE, Sotocinal SG, Tabaka JM, Wong D, van den Maagdenberg AM, Ferrari MD, 
Craig KD, Mogil JS. Coding of facial expressions of pain in the laboratory mouse. Nature methods 2010; 7: 447-449.

196. Roman J, Ritzenthaler JD, Gil-Acosta A, Rivera HN, Roser-Page S. Nicotine and fibronectin expression in lung fibroblasts: implications for tobacco-related lung tissue remodeling. FASEB journal : official publication of the Federation of American Societies for Experimental Biology 2004; 18: 1436-1438.

197. Watson WH, Burke TJ, Zelko IN, Torres-Gonzalez E, Ritzenthaler JD, Roman J. Differential Regulation of the Extracellular Cysteine/Cystine Redox State (EhCySS) by Lung Fibroblasts from Young and Old Mice. Oxidative medicine and cellular longevity 2016; 2016: 1561305.

198. Ramirez AM, Shen Z, Ritzenthaler JD, Roman J. Myofibroblast transdifferentiation in obliterative bronchiolitis: tgf-beta signaling through smad3-dependent and independent pathways. American journal of transplantation : official journal of the American Society of Transplantation and the American Society of Transplant Surgeons 2006; 6: 2080-2088.

199. Ramirez AM, Wongtrakool C, Welch T, Steinmeyer A, Zugel U, Roman J. Vitamin $\mathrm{D}$ inhibition of pro-fibrotic effects of transforming growth factor beta1 in lung fibroblasts and epithelial cells. The Journal of steroid biochemistry and molecular biology 2010; 118: 142-150.

200. Carter SL, Muller M, Manders PM, Campbell IL. Induction of the genes for Cxc19 and $\mathrm{Cxcl10}$ is dependent on IFN-gamma but shows differential cellular expression in experimental autoimmune encephalomyelitis and by astrocytes and microglia in vitro. Glia 2007; 55: 1728-1739.

201. Wang J, Nikrad MP, Travanty EA, Zhou B, Phang T, Gao B, Alford T, Ito Y, Nahreini P, Hartshorn K, Wentworth D, Dinarello CA, Mason RJ. Innate immune response of human alveolar macrophages during influenza A infection. PloS one 2012; 7: e29879.

202. Newton AH, Cardani A, Braciale TJ. The host immune response in respiratory virus infection: balancing virus clearance and immunopathology. Seminars in Immunopathology 2016; 38: 471-482.

203. Katsoulidis E, Carayol N, Woodard J, Konieczna I, Majchrzak-Kita B, Jordan A, Sassano A, Eklund EA, Fish EN, Platanias LC. Role of Schlafen 2 (SLFN2) in the generation of interferon alpha-induced growth inhibitory responses. The Journal of biological chemistry 2009; 284: 25051-25064.

204. Stegemann-Koniszewski S, Jeron A, Gereke M, Geffers R, Kroger A, Gunzer M, Bruder D. Alveolar Type II Epithelial Cells Contribute to the Anti-Influenza A Virus Response in the Lung by Integrating Pathogen- and MicroenvironmentDerived Signals. mBio 2016; 7. 
205. van Zuylen WJ, Garceau V, Idris A, Schroder K, Irvine KM, Lattin JE, Ovchinnikov DA, Perkins AC, Cook AD, Hamilton JA, Hertzog PJ, Stacey KJ, Kellie S, Hume DA, Sweet MJ. Macrophage Activation and Differentiation Signals Regulate Schlafen-4 Gene Expression: Evidence for Schlafen-4 as a Modulator of Myelopoiesis. PloS one 2011; 6: e15723.

206. Verhelst J, Parthoens E, Schepens B, Fiers W, Saelens X. Interferon-inducible protein $\mathrm{Mx} 1$ inhibits influenza virus by interfering with functional viral ribonucleoprotein complex assembly. Journal of virology 2012; 86: 13445-13455.

207. Pillai PS, Molony RD, Martinod K, Dong H, Pang IK, Tal MC, Solis AG, Bielecki P, Mohanty S, Trentalange M, Homer RJ, Flavell RA, Wagner DD, Montgomery RR, Shaw AC, Staeheli P, Iwasaki A. Mx1 reveals innate pathways to antiviral resistance and lethal influenza disease. Science (New York, NY) 2016; 352: 463 466.

208. Zhou X, Michal JJ, Zhang L, Ding B, Lunney JK, Liu B, Jiang Z. Interferon Induced IFIT Family Genes in Host Antiviral Defense. International Journal of Biological Sciences 2013; 9: 200-208.

209. Lietzen N, Ohman T, Rintahaka J, Julkunen I, Aittokallio T, Matikainen S, Nyman TA. Quantitative subcellular proteome and secretome profiling of influenza A virus-infected human primary macrophages. PLoS pathogens 2011; 7: e1001340.

210. Desai TM, Marin M, Chin CR, Savidis G, Brass AL, Melikyan GB. IFITM3 restricts influenza A virus entry by blocking the formation of fusion pores following virusendosome hemifusion. PLoS pathogens 2014; 10: e1004048.

211. Diamond MS, Farzan M. The broad-spectrum antiviral functions of IFIT and IFITM proteins. Nature reviews Immunology 2013; 13: 46-57.

212. Pinto AK, Williams GD, Szretter KJ, White JP, Proença-Módena JL, Liu G, Olejnik J, Brien JD, Ebihara H, Mühlberger E, Amarasinghe G, Diamond MS, Boon ACM. Human and Murine IFIT1 Proteins Do Not Restrict Infection of NegativeSense RNA Viruses of the Orthomyxoviridae, Bunyaviridae, and Filoviridae Families. Journal of virology 2015; 89: 9465-9476.

213. Jensen S, Thomsen AR. Sensing of RNA Viruses: a Review of Innate Immune Receptors Involved in Recognizing RNA Virus Invasion. Journal of virology 2012; 86: 2900-2910.

214. Hatesuer B, Hoang HT, Riese P, Trittel S, Gerhauser I, Elbahesh H, Geffers R, Wilk E, Schughart K. Deletion of Irf3 and Irf7 Genes in Mice Results in Altered Interferon Pathway Activation and Granulocyte-Dominated Inflammatory Responses to Influenza A Infection. Journal of innate immunity 2017; 9: 145-161.

215. Ciancanelli MJ, Huang SX, Luthra P, Garner H, Itan Y, Volpi S, Lafaille FG, Trouillet C, Schmolke M, Albrecht RA, Israelsson E, Lim HK, Casadio M, Hermesh T, Lorenzo L, Leung LW, Pedergnana V, Boisson B, Okada S, Picard C, 
Ringuier B, Troussier F, Chaussabel D, Abel L, Pellier I, Notarangelo LD, Garcia-Sastre A, Basler CF, Geissmann F, Zhang SY, Snoeck HW, Casanova JL. Infectious disease. Life-threatening influenza and impaired interferon amplification in human IRF7 deficiency. Science (New York, NY) 2015; 348: 448453.

216. Ketscher L, Hannss R, Morales DJ, Basters A, Guerra S, Goldmann T, Hausmann A, Prinz M, Naumann R, Pekosz A, Utermohlen O, Lenschow DJ, Knobeloch KP. Selective inactivation of USP18 isopeptidase activity in vivo enhances ISG15 conjugation and viral resistance. Proceedings of the National Academy of Sciences of the United States of America 2015; 112: 1577-1582.

217. Watanabe T, Tisoncik-Go J, Tchitchek N, Watanabe S, Benecke AG, Katze MG, Kawaoka Y. 1918 Influenza virus hemagglutinin (HA) and the viral RNA polymerase complex enhance viral pathogenicity, but only HA induces aberrant host responses in mice. Journal of virology 2013; 87: 5239-5254.

218. Uchida Y, Watanabe C, Takemae N, Hayashi T, Oka T, Ito T, Saito T. Identification of host genes linked with the survivability of chickens infected with recombinant viruses possessing $\mathrm{H} 5 \mathrm{~N} 1$ surface antigens from a highly pathogenic avian influenza virus. Journal of virology 2012; 86: 2686-2695.

219. Kuriakose T, Man SM, Malireddi RK, Karki R, Kesavardhana S, Place DE, Neale G, Vogel P, Kanneganti TD. ZBP1/DAI is an innate sensor of influenza virus triggering the NLRP3 inflammasome and programmed cell death pathways. Science immunology 2016; 1.

220. Kesavardhana S, Kuriakose T, Guy CS, Samir P, Malireddi RKS, Mishra A, Kanneganti TD. ZBP1/DAI ubiquitination and sensing of influenza vRNPs activate programmed cell death. The Journal of experimental medicine 2017.

221. Chin K-C, Cresswell P. Viperin (cig5), an IFN-inducible antiviral protein directly induced by human cytomegalovirus. Proceedings of the National Academy of Sciences of the United States of America 2001; 98: 15125-15130.

222. Wang X, Hinson ER, Cresswell P. The interferon-inducible protein viperin inhibits influenza virus release by perturbing lipid rafts. Cell host \& microbe 2007; 2: 96105.

223. Bering T, Carstensen MB, Rath MF. Deleting the Arntl clock gene in the granular layer of the mouse cerebellum: impact on the molecular circadian clockwork. Journal of neurochemistry 2017.

224. Sundar IK, Ahmad T, Yao H, Hwang JW, Gerloff J, Lawrence BP, Sellix MT, Rahman I. Influenza A virus-dependent remodeling of pulmonary clock function in a mouse model of COPD. Scientific reports 2015; 4: 9927. 
225. Tregoning JS, Schwarze J. Respiratory Viral Infections in Infants: Causes, Clinical Symptoms, Virology, and Immunology. Clinical microbiology reviews 2010; 23: 74-98.

226. Bautista E, Chotpitayasunondh T, Gao Z, Harper SA, Shaw M, Uyeki TM, Zaki SR, Hayden FG, Hui DS, Kettner JD, Kumar A, Lim M, Shindo N, Penn C, Nicholson KG. Clinical aspects of pandemic 2009 influenza A (H1N1) virus infection. The New England journal of medicine 2010; 362: 1708-1719.

227. WHO. Recommended composition of influenza virus vaccines for use in the 2010 influenza season (southern hemisphere winter). 2009 Oct 09 [cited 84 41].

228. Cheng VC, To KK, Tse H, Hung IF, Yuen KY. Two years after pandemic influenza A/2009/H1N1: what have we learned? Clinical microbiology reviews 2012; 25: 223-263.

229. Neumann G, Kawaoka Y. The first influenza pandemic of the new millennium. Influenza and Other Respiratory Viruses 2011; 5: 157-166.

230. Cell culture as a substrate for the production of influenza vaccines: memorandum from a WHO meeting. Bulletin of the World Health Organization 1995; 73: 431435 .

231. Wasik MA, Eichwald L, Genzel Y, Reichl U. Cell culture-based production of defective interfering particles for influenza antiviral therapy. Applied Microbiology and Biotechnology 2018; 102: 1167-1177.

232. Cottey R, Rowe CA, Bender BS. Influenza virus. Current protocols in immunology 2001; Chapter 19: Unit 19.11.

233. Blazejewska P, Koscinski L, Viegas N, Anhlan D, Ludwig S, Schughart K. Pathogenicity of different PR8 influenza A virus variants in mice is determined by both viral and host factors. Virology 2011; 412: 36-45.

234. Pica N, Iyer A, Ramos I, Bouvier NM, Fernandez-Sesma A, Garcia-Sastre A, Lowen AC, Palese P, Steel J. The DBA.2 mouse is susceptible to disease following infection with a broad, but limited, range of influenza A and B viruses. Journal of virology 2011; 85: 12825-12829.

235. Hirst GK. STUDIES ON THE MECHANISM OF ADAPTATION OF INFLUENZA VIRUS TO MICE. The Journal of experimental medicine 1947; 86: 357-366.

236. Raut S, Hurd J, Blandford G, Heath RB, Cureton RJ. The pathogenesis of infections of the mouse caused by virulent and avirulent variants of an influenza virus. Journal of medical microbiology 1975; 8: 127-136.

237. Jolly L, Stavrou A, Vanderstoken G, Meliopoulos VA, Habgood A, Tatler AL, Porte J, Knox A, Weinreb P, Violette S, Hussell T, Kolb M, Stampfli MR, SchultzCherry S, Jenkins G. Influenza promotes collagen deposition via alphavbeta6 
integrin-mediated transforming growth factor beta activation. The Journal of biological chemistry 2014; 289: 35246-35263.

238. Leung HS, Li OT, Chan RW, Chan MC, Nicholls JM, Poon LL. Entry of influenza A Virus with a alpha2,6-linked sialic acid binding preference requires host fibronectin. Journal of virology 2012; 86: 10704-10713.

239. Klein SL, Hodgson A, Robinson DP. Mechanisms of sex disparities in influenza pathogenesis. Journal of leukocyte biology 2012; 92: 67-73.

240. Lorenzo ME, Hodgson A, Robinson DP, Kaplan JB, Pekosz A, Klein SL. Antibody responses and cross protection against lethal influenza $A$ viruses differ between the sexes in C57BL/6 mice. Vaccine 2011; 29: 9246-9255.

241. Reinhard C, Eder G, Fuchs H, Ziesenis A, Heyder J, Schulz H. Inbred strain variation in lung function. Mammalian genome : official journal of the International Mammalian Genome Society 2002; 13: 429-437.

242. Voltz JW, Card JW, Carey MA, Degraff LM, Ferguson CD, Flake GP, Bonner JC, Korach KS, Zeldin DC. Male sex hormones exacerbate lung function impairment after bleomycin-induced pulmonary fibrosis. American journal of respiratory cell and molecular biology 2008; 39: 45-52.

243. Lingappan K, Jiang W, Wang L, Moorthy B. Sex-specific differences in neonatal hyperoxic lung injury. American journal of physiology Lung cellular and molecular physiology 2016; 311: L481-493.

244. Schaubel D, Johansen H, Dutta M, Desmeules M, Becker A, Mao Y. Neonatal characteristics as risk factors for preschool asthma. The Journal of asthma : official journal of the Association for the Care of Asthma 1996; 33: 255-264.

245. Larcombe AN, Foong RE, Bozanich EM, Berry LJ, Garratt LW, Gualano RC, Jones JE, Dousha LF, Zosky GR, Sly PD. Sexual dimorphism in lung function responses to acute influenza A infection. Influenza Other Respir Viruses 2011; 5: 334-342.

246. Irvin CG, Bates JH. Measuring the lung function in the mouse: the challenge of size. Respiratory research 2003; 4: 4.

247. Centers for Disease C, Prevention, National Center for Chronic Disease P, Health P, Office on S, Health. Publications and Reports of the Surgeon General. How Tobacco Smoke Causes Disease: The Biology and Behavioral Basis for SmokingAttributable Disease: A Report of the Surgeon General. Atlanta (GA): Centers for Disease Control and Prevention (US); 2010.

248. Cardinale A, Nastrucci C, Cesario A, Russo P. Nicotine: specific role in angiogenesis, proliferation and apoptosis. Critical reviews in toxicology 2012; 42: 68-89. 
249. Razani-Boroujerdi S, Singh SP, Knall C, Hahn FF, Pena-Philippides JC, Kalra R, Langley RJ, Sopori ML. Chronic nicotine inhibits inflammation and promotes influenza infection. Cellular immunology 2004; 230: 1-9.

250. Verhoeven D, Teijaro JR, Farber DL. Pulse-oximetry accurately predicts lung pathology and the immune response during influenza infection. Virology 2009; 390: 151-156.

251. Sharafkhaneh A, Hanania NA, Kim V. Pathogenesis of Emphysema: From the Bench to the Bedside. Proceedings of the American Thoracic Society 2008; 5: 475-477.

252. Totti N, 3rd, McCusker KT, Campbell EJ, Griffin GL, Senior RM. Nicotine is chemotactic for neutrophils and enhances neutrophil responsiveness to chemotactic peptides. Science (New York, NY) 1984; 223: 169-171.

253. Bradley LM, Douglass MF, Chatterjee D, Akira S, Baaten BJG. Matrix Metalloprotease 9 Mediates Neutrophil Migration into the Airways in Response to Influenza Virus-Induced Toll-Like Receptor Signaling. PLoS pathogens 2012; 8: e1002641.

254. Taraseviciene-Stewart L, Voelkel NF. Molecular pathogenesis of emphysema. The Journal of clinical investigation 2008; 118: 394-402.

255. Armstrong LW, Rom WN, Martiniuk FT, Hart D, Jagirdar J, Galdston M. Nicotine enhances expression of the neutrophil elastase gene and protein in a human myeloblast/promyelocyte cell line. American journal of respiratory and critical care medicine 1996; 154: 1520-1524.

256. Metzger MJ, Halperin AC, Manhart LE, Hawes SE. Association of maternal smoking during pregnancy with infant hospitalization and mortality due to infectious diseases. The Pediatric infectious disease journal 2013; 32: e1-7.

257. Shaheen SO, Sterne JA, Tucker JS, Florey CD. Birth weight, childhood lower respiratory tract infection, and adult lung function. Thorax 1998; 53: 549-553.

258. Cook DG, Strachan DP. Health effects of passive smoking-10: Summary of effects of parental smoking on the respiratory health of children and implications for research. Thorax 1999; 54: 357-366.

259. Barker DJ, Godfrey KM, Fall C, Osmond C, Winter PD, Shaheen SO. Relation of birth weight and childhood respiratory infection to adult lung function and death from chronic obstructive airways disease. BMJ (Clinical research ed) 1991; 303: 671-675.

260. Taylor B, Wadsworth J. Maternal smoking during pregnancy and lower respiratory tract illness in early life. Archives of disease in childhood 1987; 62: 786-791.

261. Hajos M, Rogers BN. Targeting alpha7 nicotinic acetylcholine receptors in the treatment of schizophrenia. Current pharmaceutical design 2010; 16: 538-554. 
262. Zia S, Ndoye A, Nguyen VT, Grando SA. Nicotine enhances expression of the alpha 3 , alpha 4, alpha 5, and alpha 7 nicotinic receptors modulating calcium metabolism and regulating adhesion and motility of respiratory epithelial cells. Research communications in molecular pathology and pharmacology 1997; 97: 243-262.

263. Hiemke C, Stolp M, Reuss S, Wevers A, Reinhardt S, Maelicke A, Schlegel S, Schroder H. Expression of alpha subunit genes of nicotinic acetylcholine receptors in human lymphocytes. Neuroscience letters 1996; 214: 171-174.

264. Liu J, McGlinn AM, Fernandes A, Milam AH, Strang CE, Andison ME, Lindstrom JM, Keyser KT, Stone RA. Nicotinic acetylcholine receptor subunits in rhesus monkey retina. Investigative ophthalmology \& visual science 2009; 50: 14081415 .

265. Aztiria EM, Sogayar MC, Barrantes FJ. Expression of a neuronal nicotinic acetylcholine receptor in insect and mammalian host cell systems. Neurochemical research 2000; 25: 171-180.

266. Belmonte KE. Cholinergic pathways in the lungs and anticholinergic therapy for chronic obstructive pulmonary disease. Proceedings of the American Thoracic Society 2005; 2: 297-304; discussion 311-292.

267. Burri PH. Structural aspects of postnatal lung development - alveolar formation and growth. Biology of the neonate 2006; 89: 313-322.

268. Jensen K, Nizamutdinov D, Guerrier M, Afroze S, Dostal D, Glaser S. General mechanisms of nicotine-induced fibrogenesis. FASEB journal : official publication of the Federation of American Societies for Experimental Biology 2012; 26: 4778-4787.

269. Lavezzi AM, Corna MF, Alfonsi G, Matturri L. Possible role of the $\alpha 7$ nicotinic receptors in mediating nicotine's effect on developing lung - implications in unexplained human perinatal death. BMC Pulmonary Medicine 2014; 14: 11-11.

270. Orr-Urtreger A, Goldner FM, Saeki M, Lorenzo I, Goldberg L, De Biasi M, Dani JA, Patrick JW, Beaudet AL. Mice deficient in the alpha7 neuronal nicotinic acetylcholine receptor lack alpha-bungarotoxin binding sites and hippocampal fast nicotinic currents. The Journal of neuroscience : the official journal of the Society for Neuroscience 1997; 17: 9165-9171.

271. Pohanka M. Alpha7 Nicotinic Acetylcholine Receptor Is a Target in Pharmacology and Toxicology. International Journal of Molecular Sciences 2012; 13: 22192238 .

272. Manti S, Cuppari C, Lanzafame A, Salpietro C, Betta P, Leonardi S, Perez MK, Piedimonte G. Detection of respiratory syncytial virus (RSV) at birth in a newborn with respiratory distress. Pediatric pulmonology 2017; 52: E81-e84. 
273. Cho SH, Stanciu LA, Holgate ST, Johnston SL. Increased interleukin-4, interleukin5 , and interferon-gamma in airway CD4+ and CD8+ T cells in atopic asthma. American journal of respiratory and critical care medicine 2005; 171: 224-230.

274. Lloyd CM, Hessel EM. Functions of T cells in asthma: more than just $\mathrm{T}(\mathrm{H}) 2$ cells. Nature reviews Immunology 2010; 10: 838-848.

275. Akbari O, Stock P, Meyer E, Kronenberg M, Sidobre S, Nakayama T, Taniguchi M, Grusby MJ, DeKruyff RH, Umetsu DT. Essential role of NKT cells producing IL4 and IL-13 in the development of allergen-induced airway hyperreactivity. Nature medicine 2003; 9: 582-588.

276. Nagata Y, Kamijuku H, Taniguchi M, Ziegler S, Seino K. Differential role of thymic stromal lymphopoietin in the induction of airway hyperreactivity and Th2 immune response in antigen-induced asthma with respect to natural killer $\mathrm{T}$ cell function. International archives of allergy and immunology 2007; 144: 305-314.

277. Reynolds C, Barkans J, Clark P, Kariyawasam H, Altmann D, Kay B, Boyton R. Natural killer $\mathrm{T}$ cells in bronchial biopsies from human allergen challenge model of allergic asthma. The Journal of allergy and clinical immunology 2009; 124: $860-862$; author reply 862 .

278. Matangkasombut P, Marigowda G, Ervine A, Idris L, Pichavant M, Kim HY, Yasumi T, Wilson SB, DeKruyff RH, Faul JL, Israel E, Akbari O, Umetsu DT. Natural killer T cells in the lungs of patients with asthma. The Journal of allergy and clinical immunology 2009; 123: 1181-1185.

279. Kim HY, DeKruyff RH, Umetsu DT. The many paths to asthma: phenotype shaped by innate and adaptive immunity. Nature immunology 2010; 11: 577-584.

280. Paul WE, Zhu J. How are $\mathrm{T}(\mathrm{H}) 2$-type immune responses initiated and amplified? Nature reviews Immunology 2010; 10: 225-235.

281. Larche M, Robinson DS, Kay AB. The role of T lymphocytes in the pathogenesis of asthma. The Journal of allergy and clinical immunology 2003; 111: 450-463; quiz 464.

282. Froidure A, Mouthuy J, Durham SR, Chanez P, Sibille Y, Pilette C. Asthma phenotypes and IgE responses. The European respiratory journal 2016; 47: 304319.

283. Saenz SA, Siracusa MC, Perrigoue JG, Spencer SP, Urban JF, Jr., Tocker JE, Budelsky AL, Kleinschek MA, Kastelein RA, Kambayashi T, Bhandoola A, Artis D. IL25 elicits a multipotent progenitor cell population that promotes $\mathrm{T}(\mathrm{H}) 2$ cytokine responses. Nature 2010; 464: 1362-1366.

284. Moro K, Yamada T, Tanabe M, Takeuchi T, Ikawa T, Kawamoto H, Furusawa J, Ohtani M, Fujii H, Koyasu S. Innate production of $\mathrm{T}(\mathrm{H}) 2$ cytokines by adipose tissue-associated c-Kit(+)Sca-1(+) lymphoid cells. Nature 2010; 463: 540-544. 
285. Ostroukhova M, Seguin-Devaux C, Oriss TB, Dixon-McCarthy B, Yang L, Ameredes BT, Corcoran TE, Ray A. Tolerance induced by inhaled antigen involves CD4(+) T cells expressing membrane-bound TGF-beta and FOXP3. The Journal of clinical investigation 2004; 114: 28-38.

286. Kearley J, Barker JE, Robinson DS, Lloyd CM. Resolution of airway inflammation and hyperreactivity after in vivo transfer of CD4+CD25+ regulatory $\mathrm{T}$ cells is interleukin 10 dependent. The Journal of experimental medicine 2005; 202: 15391547.

287. Leech MD, Benson RA, De Vries A, Fitch PM, Howie SE. Resolution of Der p1induced allergic airway inflammation is dependent on CD4+CD25+Foxp3+ regulatory cells. Journal of immunology (Baltimore, Md : 1950) 2007; 179: 70507058 .

288. Joetham A, Takeda K, Taube C, Miyahara N, Matsubara S, Koya T, Rha YH, Dakhama A, Gelfand EW. Naturally occurring lung CD4(+)CD25(+) T cell regulation of airway allergic responses depends on IL-10 induction of TGF-beta. Journal of immunology (Baltimore, Md : 1950) 2007; 178: 1433-1442.

289. Strickland DH, Stumbles PA, Zosky GR, Subrata LS, Thomas JA, Turner DJ, Sly PD, Holt PG. Reversal of airway hyperresponsiveness by induction of airway mucosal CD4+CD25+ regulatory T cells. The Journal of experimental medicine 2006; 203: 2649-2660.

290. Monticelli LA, Sonnenberg GF, Artis D. Innate lymphoid cells: critical regulators of allergic inflammation and tissue repair in the lung. Current opinion in immunology 2012; 24: 284-289.

291. Monticelli LA, Sonnenberg GF, Abt MC, Alenghat T, Ziegler CG, Doering TA, Angelosanto JM, Laidlaw BJ, Yang CY, Sathaliyawala T, Kubota M, Turner D, Diamond JM, Goldrath AW, Farber DL, Collman RG, Wherry EJ, Artis D. Innate lymphoid cells promote lung-tissue homeostasis after infection with influenza virus. Nature immunology 2011; 12: 1045-1054.

292. Schuger L, Johnson GR, Gilbride K, Plowman GD, Mandel R. Amphiregulin in lung branching morphogenesis: interaction with heparan sulfate proteoglycan modulates cell proliferation. Development (Cambridge, England) 1996; 122: 1759-1767.

293. Kim KW, Jee HM, Park YH, Choi BS, Sohn MH, Kim KE. Relationship between amphiregulin and airway inflammation in children with asthma and eosinophilic bronchitis. Chest 2009; 136: 805-810. 


\title{
CURRICULUM VITA
}

\author{
Ryan Carroll McAllister \\ April 3, 1988 (Detroit, Michigan) \\ Rcmcallister2020@gmail.com \\ University of Louisville \\ 505 S. Hancock \#527C \\ Louisville, KY 40202
}

\section{EDUCATION}

2018 Ph.D. Pharmacology and Toxicology University of Louisville "The Effects of Perinatal Nicotine with or without Early Life pH1N1 on Murine Lung Dysfunction in Adulthood"

2014 M.S. Pharmacology and Toxicology University of Louisville "Development of Models for the Study of the Molecular Mechanisms of Host Restriction and Adaptation of Hantavirus"

2010 B.S. Biochemistry Northern Michigan University, Pre-Medical Program

\section{PUBLICATIONS}

$\underline{\text { Published }}$

McAllister RC and Jonsson CB. Hantaviruses: past, present and future. Future Virology 2014; 9.

Camp JV, Chu, YK, Chung DH, McAllister RC, Adcock RS, Gerlach RL, Wiemken, T, Peyrani, P, Ramirez JA, Summersgill JT, Jonsson CB. Phenotypic differences in virulence and immune response in closely related clinical isolates of influenza A 2009 H1N1 pandemic viruses in mice. PLoS One 2013; 8: e56602

Chung D-H, Västermark Å, Camp JV, McAllister R, Remold SK, Chu Y-K, Bruder C, Jonsson $\mathrm{CB}$. The murine model for Hantaan virus-induced lethal disease shows two distinct paths in viral evolutionary trajectory with and without ribavirin treatment. Journal of Virology. Journal of Virology 2013; 87: 10997-11007. 


\section{$\underline{\text { Submitted }}$}

McAllister RC, Torres-Gonzalez E, Rai JP, Ritzenthaler JD, Hoyle GW, Roman J. Perinatal Nicotine Exposure Leads to Functional Abnormalities in the Murine Adult Lung. American Journal of Respiratory Cell and Molecular Biology 2018.

Owen RD, Rodriguez L, Martinez Bruyn VJ, Sage R, Camp JV, McAllister RC, Jonsson CB. Microsympatry and Microhabitat Selection of Sigmodontine Rodents in an Atlantic Forest - Cerrado Interface. Mastozoología Neotropical 2018.

Eastwood G, Chu YK, Sawyer AM, Owen RD, Taylor MK, Valdivieso L, Sage RD, Yu A, Camp JV, Goodin DG, Martinez Bruyn VJ, McAllister RC, Rodriguez LD, Williams EP, Jonsson CB. Habitat, species richness and hantaviruses of Sigmodontine species within the Interior Atlantic Forest, Paraguay. PLOS Neglected Tropical Diseases 2018.

\section{$\underline{\text { In Preparation }}$}

McAllister RC, Torres-Gonzalez E, Rai JP, Ritzenthaler JD, Rai SN, Hoyle GW, Roman J. A Murine Model for Early Life Influenza A H1N1 Pandemic 2009 Infection. Journal of General Virology 2018.

McAllister RC, Torres-Gonzalez E, Rai JP, Ritzenthaler JD, Hoyle GW, Roman J. Perinatal Nicotine Exposure Accompanied with Early Life pH1N1 Infection Leads to Murine Adult Lung Dysfunction. TBD 2018.

McAllister RC \& Roman J. Asthma and COPD: The Disease, Treatment, Diagnosis, and Models for Their Study. TBD 2018.

\section{POSTER AND OTHER PRESENTATIONS}

McAllister RC, Torres-Gonzalez E, Ritzenthaler JD, Hoyle GW, Roman J. American Thoracic Society Annual National Conference (San Diego, California 5/23/2018) "Perinatal Nicotine Exposure Along with Early Life Influenza A, 2009 Pandemic H1N1 (Swine Flu) Infection, Leads to Lung Dysfunction in Adulthood"

McAllister RC \& Jonsson CB. American Society of Virology Annual National Conference (Fort Collins, Colorado 6/23/2014) "A new in vitro primary lung microvascular endothelial cell (L-MVEC) model to study New World hantavirus infections" - Travel Grant Recipient

Research Louisville 2012-2017 (Louisville, KY)

\section{PROFESSIONAL MEMBERSHIPS}

The Association for Biosafety and Biosecurity (2018) 
Sean Kaufman Lunch Break Series Participant (2018)

Student Member, Center of Predictive Medicine for Biodefense and Emerging Infectious Diseases (2012-2018)

American Society for Virology (2013-2015)

\section{EDUCATIONAL ACTIVITIES}

Louisville Regional Science Fair Judge (2013-2015)

Student Assistant for General Virology Course (2013-2014)

Organic Chemistry Tutor (2008-2010)

\section{TRAININGS/CERTIFICATIONS}

FBI Security Risk Assessment \#RM-041236 (2012-2015)

Select Agent Training (2013-2015)

ATL1 Training (2013-2015)

NIH Guidelines (2012-2018)

Bloodborne Pathogens (2012-2018)

General Laboratory Safety and Hazardous Waste (2010-2018)

Basic Biosafety (2012-2018 